Escuela Técnica Superior de Ingenieros Navales (ETSIN)

Universidad Politécnica de Madrid (UPM)

\title{
A TIME-DOMAIN FINITE ELEMENT METHOD FOR \\ SEAKEEPING AND WAVE \\ RESISTANCE PROBLEMS
}

\author{
Borja Serván Camas \\ Ingeniero Naval \\ Master of Science in Civil Engineering \\ Doctor of Philosophy in Civil Engineering
}

Supervisors:

Julio García Espinosa

Ingeniero Naval

Doctor Ingeniero de Caminos, Canales y Puertos

Antonio Souto Iglesias

Ingeniero Naval

Doctor Ingeniero Naval 


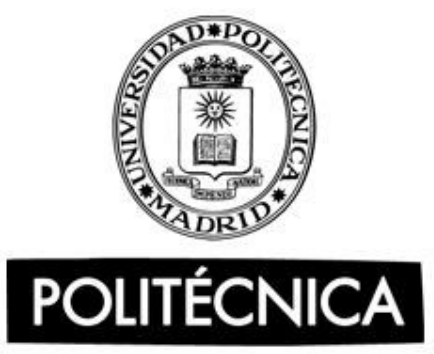

Tribunal nombrado por el Mgfco. y Excemo. Sr. Rector de la Universidad Politécnica de Madrid, el día. de de $20 \ldots$.

Presidente:

Vocal:

Vocal:

Vocal:

Secretario:

Suplente:

Suplente:

Realizado el acto de defensa y lectura de la Tesis el día de. de 20 ...... en la E.T.S.I./Facultad

Calificación

EL PRESIDENTE LOS VOCALES

EL SECRETARIO 


\section{DEDICATION}

To my beloved father,

Angel Serván Martinez.

Rest in peace. 


\section{ACKNOWLEDGEMENTS}

I am very grateful to my supervisors Professor Julio García Espinosa and Professor Antonio Souto Iglesias for their patience, confidence in my capabilities, guidance, and help towards the completion of this thesis.

I thank CIMNE, my current work place, and especially Prof. Eugenio Oñate, for giving me the opportunity to develop a professional career as a researcher. Thanks to CIMNE, the work of this thesis has been materialized in SeaFEM.

I am also very grateful to all the CompassIS staff, for their work in the development of SeaFEM, which indirectly helped in the progress of my thesis. I am especially grateful to Daniel Sá, Ovidi Casals, and Rosa Peyrau.

Thanks to my collaborators Carlos López, Prashanth Nandukandi, Daniel Di Capua, Augusto Maidana, José Enrique Gutierrez, Jonathan Colom, Pere-Andreu Ubach, and Jose Luis Cercós for their contribution to this thesis in one way or another.

And finally, but not less, thanks to all my office mates at the NT3 building, for making my everyday life pleasant and enjoyable, which indubitably helped to the development of my work.

This work was partially supported by the Office of Naval Research Global (USA) by the Navy Grant N62909-10-1-7053.

This work was partially supported by ABENGOA by a number of different collaborative projects.

This work was partially supported by the "Ministerio de Economía y Competitividad" by the XSheaks project ENE2014-59194-C2-1-R.

The author gratefully acknowledges the support of NVIDIA Corporation with the donation of the Tesla K20 GPU used in this thesis. 


\section{Table of contents}

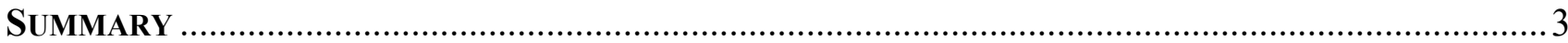

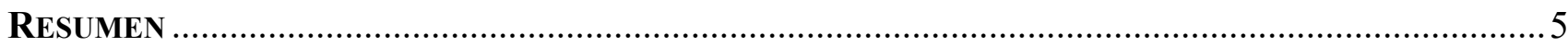

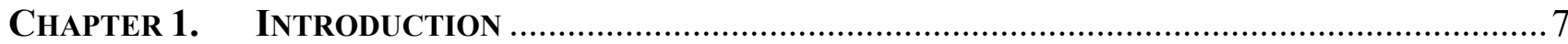

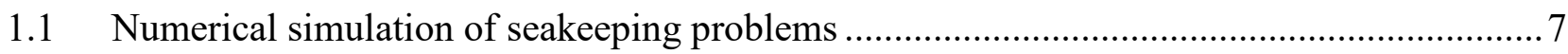

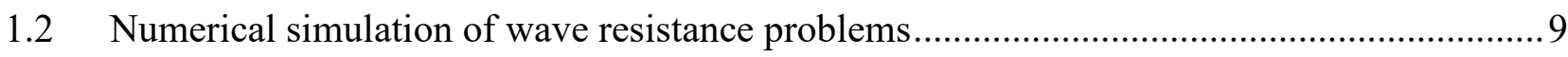

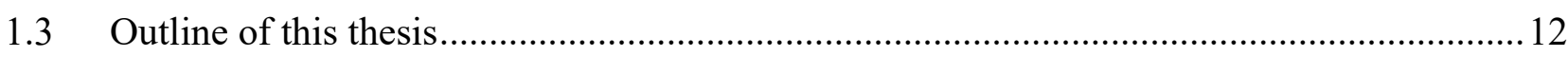

PART I: MATHEMATICAL AND NUMERICAL MODELS ............................................... 13

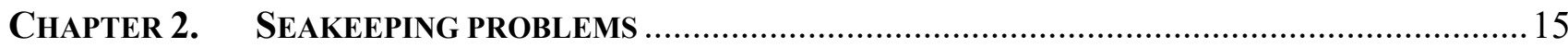

2.1 Mathematical model for seakeeping problems ........................................................ 15

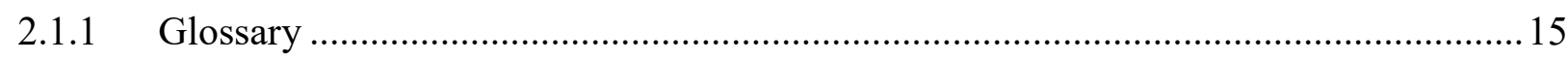

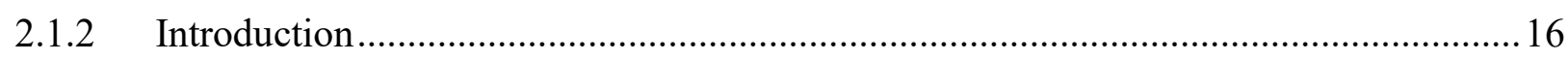

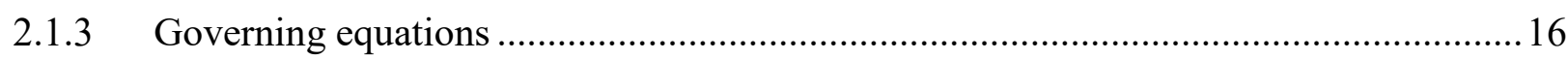

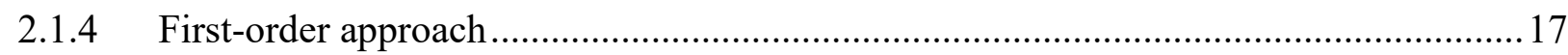

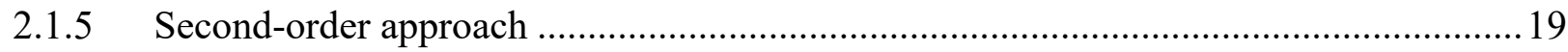

2.2 Numerical models for seakeeping problems …...........................................................22

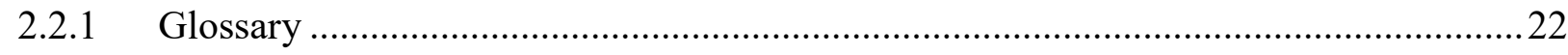

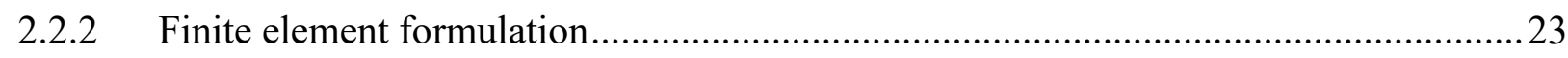

2.2.3 Free surface boundary conditions ..................................................................... 23

2.2.4 Radiation condition and wave absorption.............................................................24

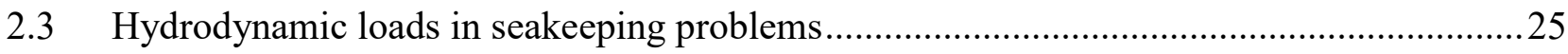

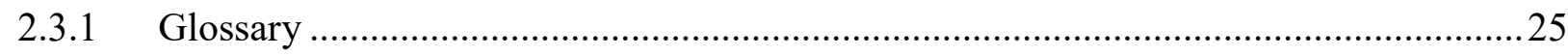

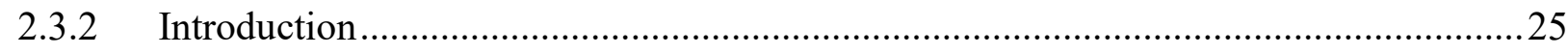

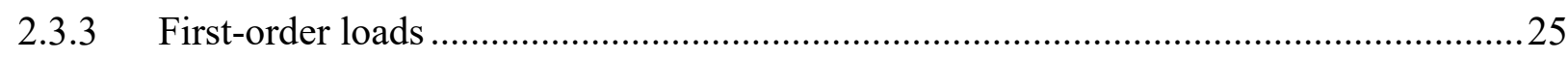

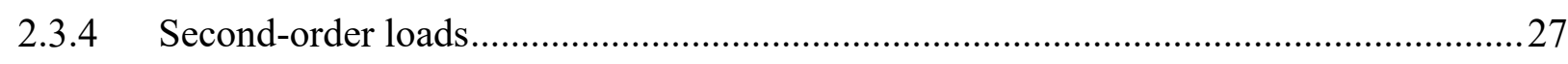

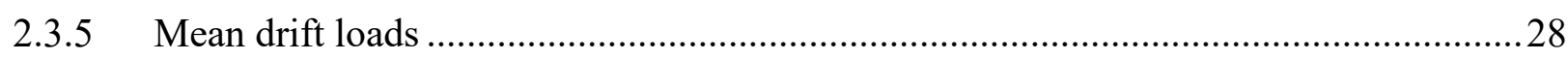

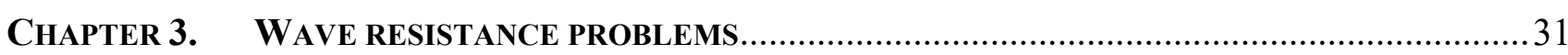

3.1 Mathematical models for wave resistance problems.................................................... 31

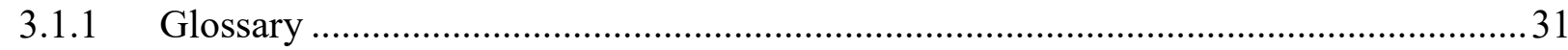

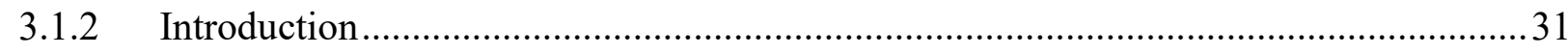

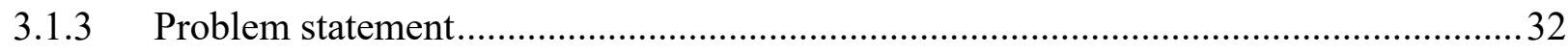

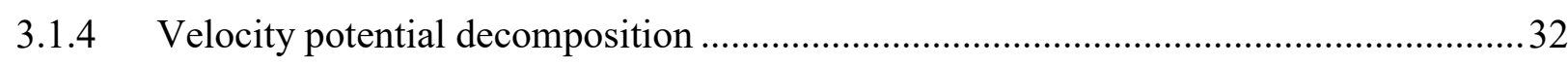

3.1.5 Governing equations in a moving frame of reference ................................................ 34 


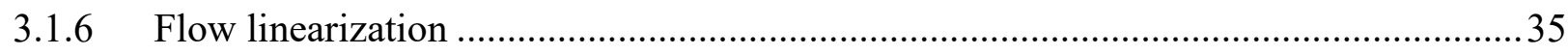

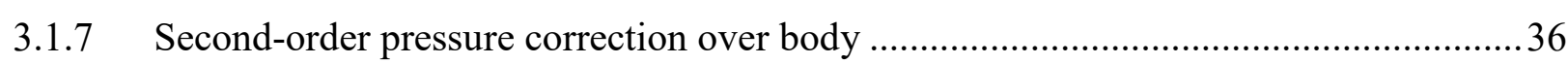

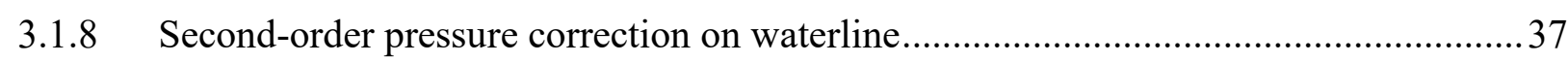

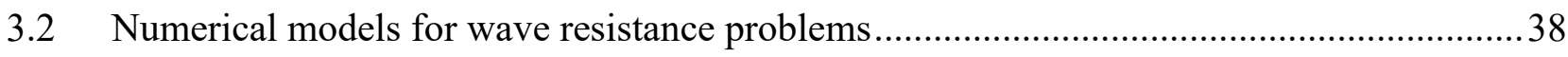

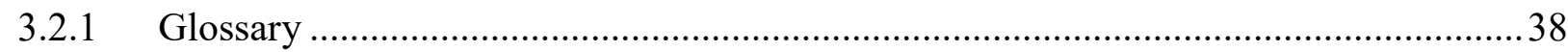

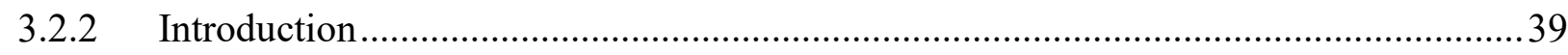

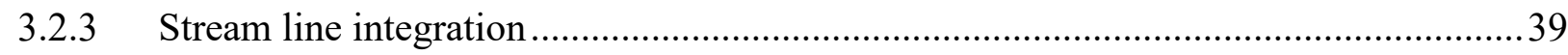

3.2.4 Streamline-Upwind Petrov-Galerkin (SUPG) formulation ........................................4 41

3.3 Hydrodynamic loads on bodies for wave resistance problems ....................................... 43

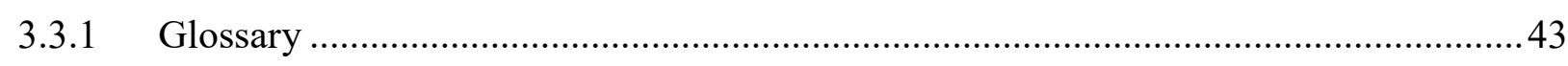

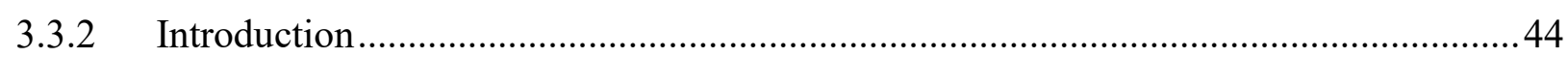

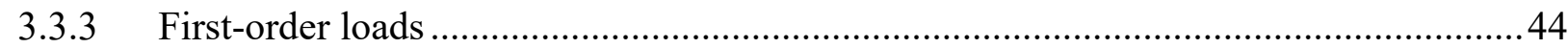

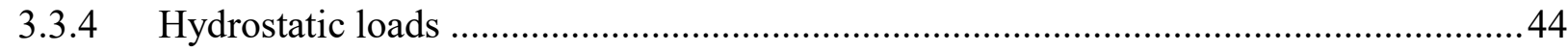

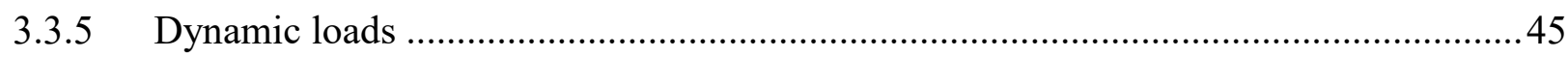

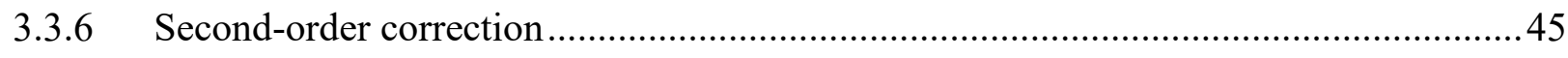

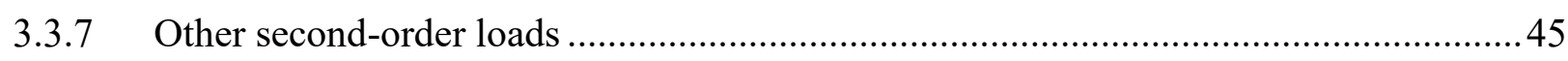

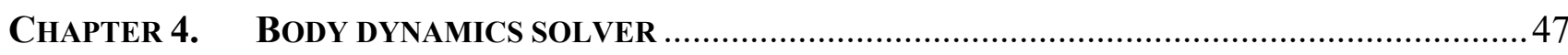

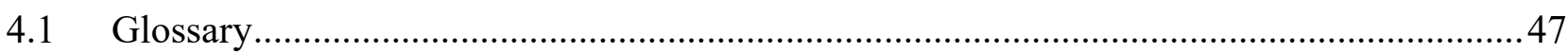

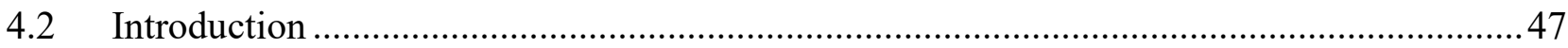

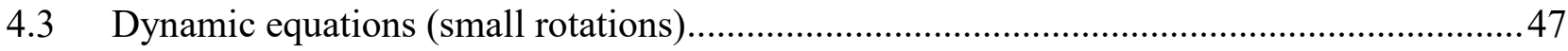

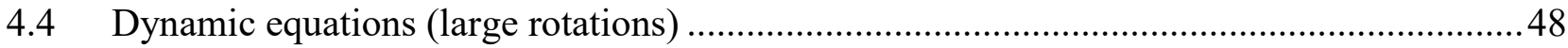

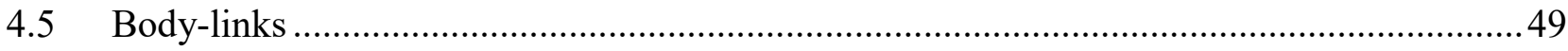

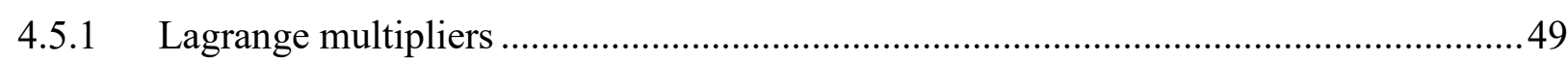

4.5.2 Body-links with Alpha Bossak-Newmark integration ...............................................50

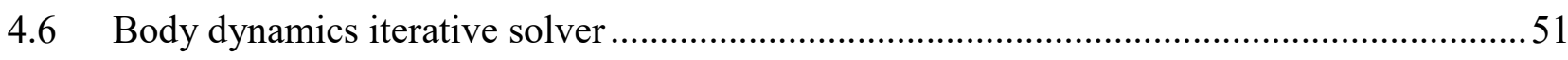

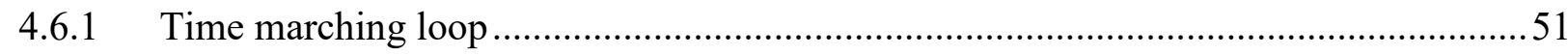

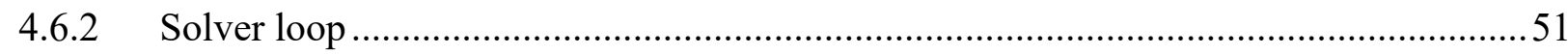

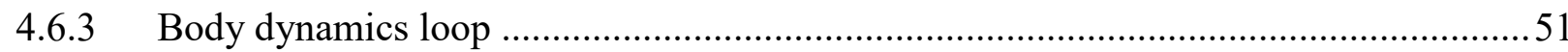

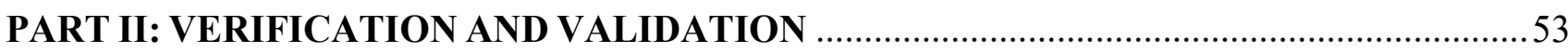

CHAPTER 5. SEAKEEPING: VERIFICATION AND VALIDATION...................................................5

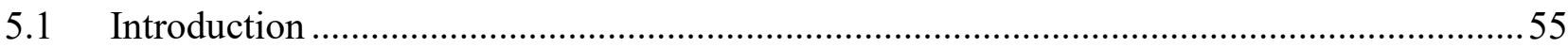

5.2 Verification case: Waves refracted by a vertical circular cylinder ..................................55

5.3 Verification case: Standing wave dissipation by pressure .............................................58

5.3.1 First-order analytical solution for pressure based wave dissipation ..........................58

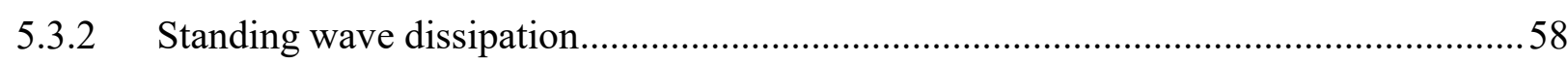

5.4 Validation case: Seakeeping of a GVA 4000 Semisubmersible platform ..........................59 


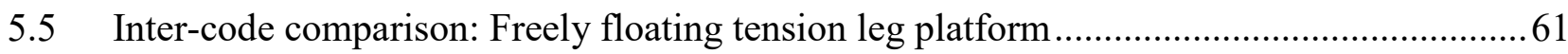

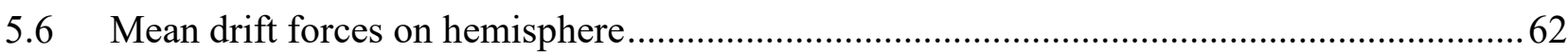

5.7 Diffraction of second-order waves by semi-submerged horizontal rectangular cylinder .....65

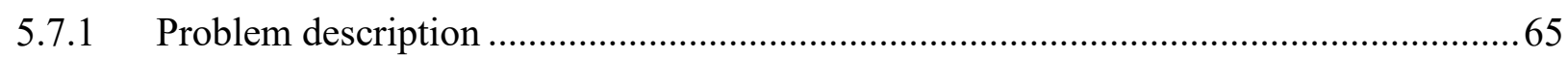

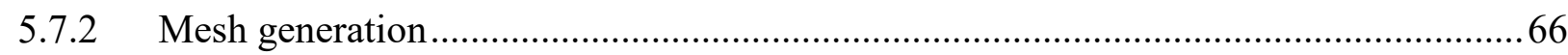

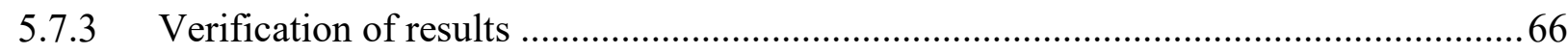

CHAPTER 6. WAVE RESISTANCE: VERIFICATION AND VALIDATION ..........................................69

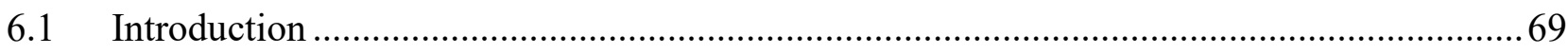

6.2 Wave making resistance of an elliptic pressure distribution...........................................69

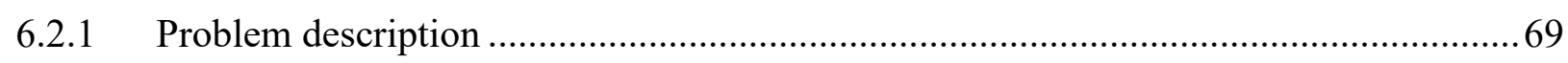

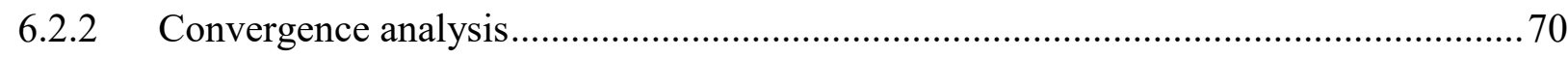

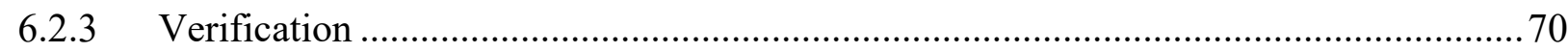

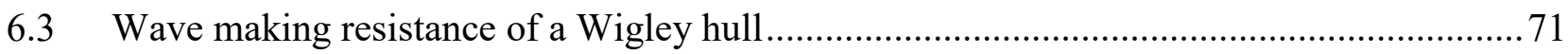

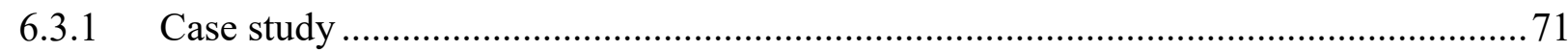

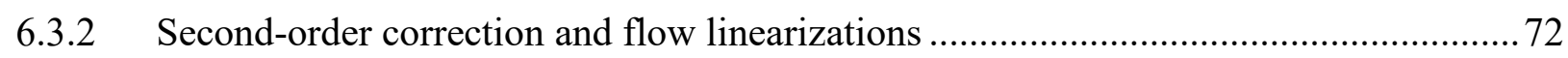

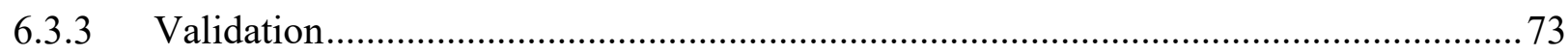

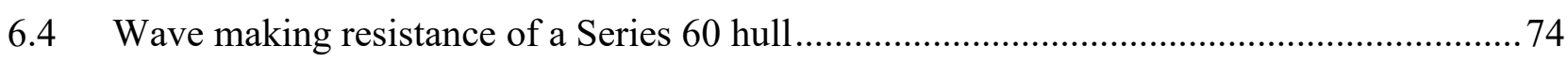

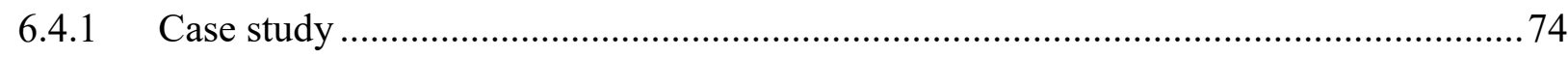

6.4.2 Second-order correction and flow linearization..................................................... 75

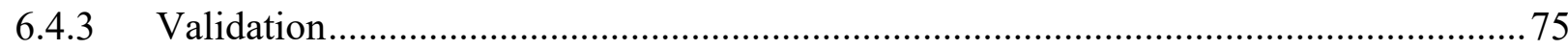

6.5 Added resistance in waves of four modified Wigley hulls ............................................. 77

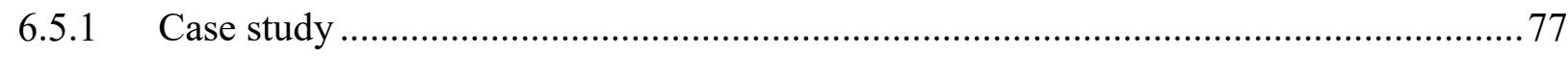

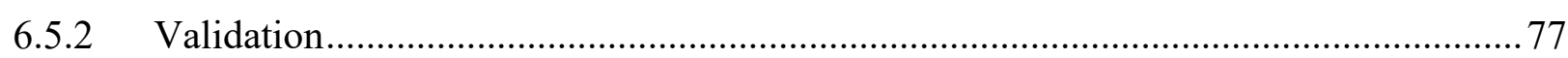

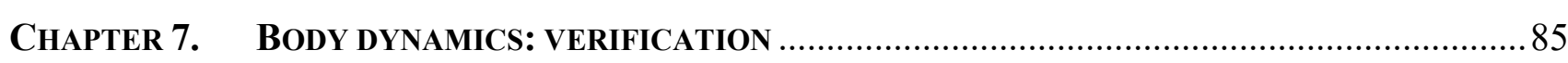

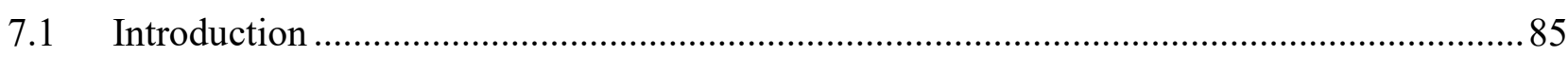

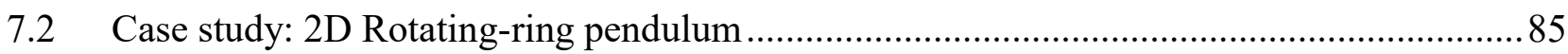

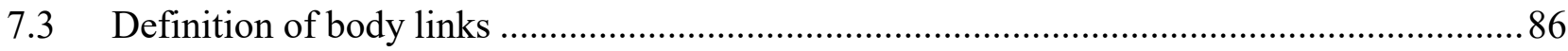

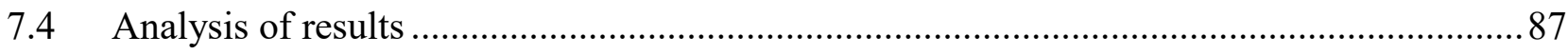

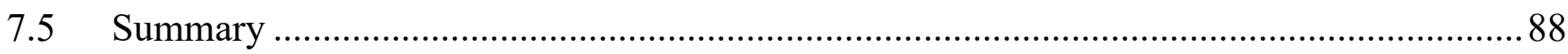

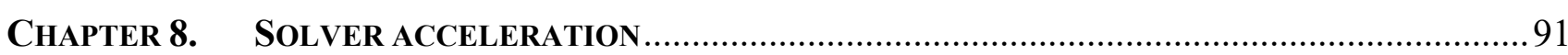

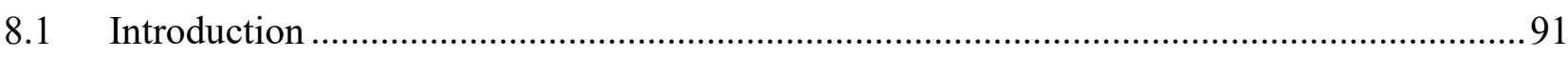

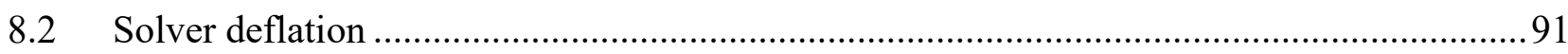

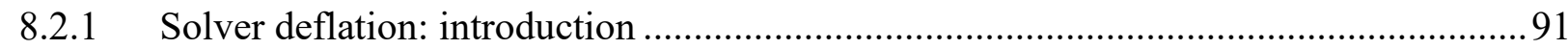

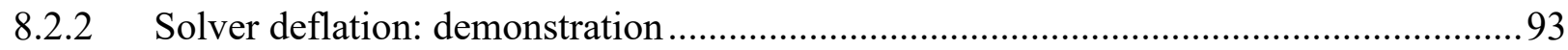

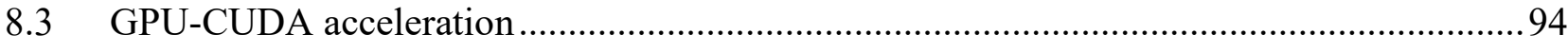

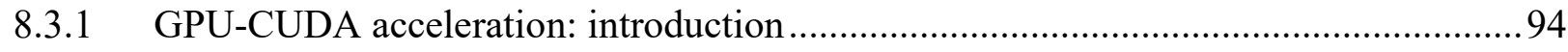

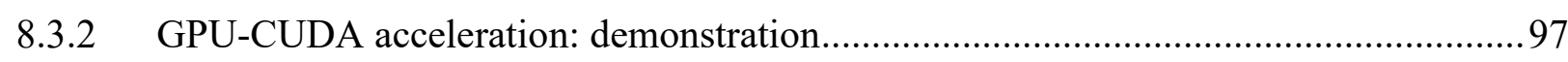

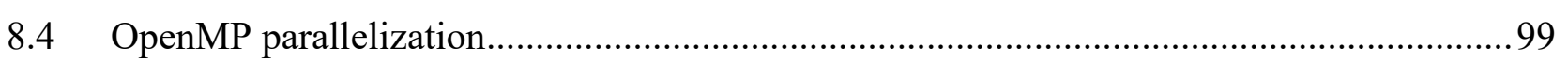


CHAPTER 9. ANALYSIS OF A SURFACE EFFECT SHIP SEAL DYNAMICS ..................................... 107

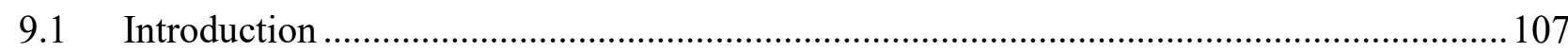

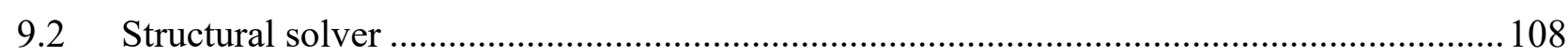

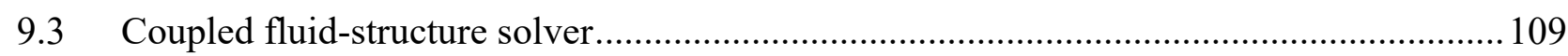

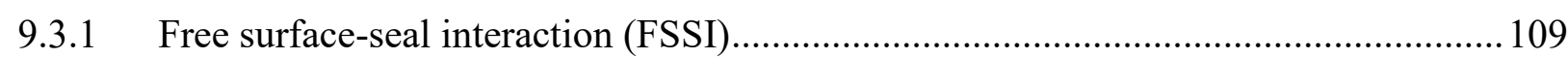

9.3.2 Free surface-flexible seas coupling algorithm .................................................... 110

9.4 Validation of FSSI algorithm with flexible bow seals ................................................ 113

9.4.1 Validation: experiments at University of Michigan.................................................. 113

9.4.2 Validation: experiments at the U.S Navy's Large Cavitation Channel (LCC)............118

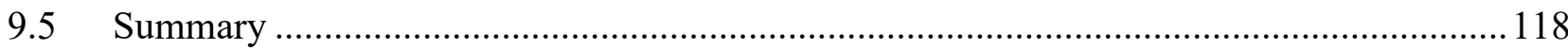

CHAPTER 10. ANALYSIS OF COUPLED SEAKEEPING - SLOSHING PROBLEMS BY FEM-SPH

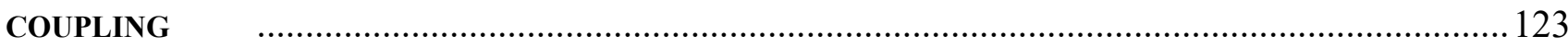

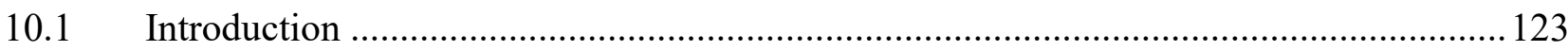

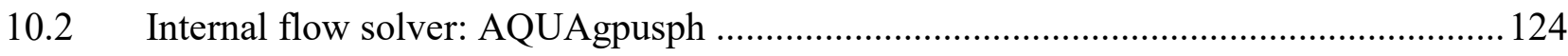

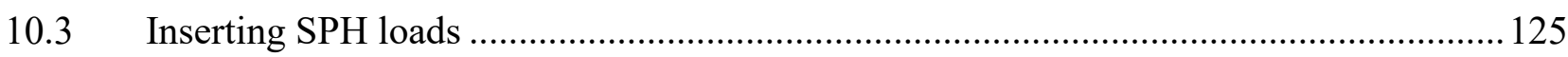

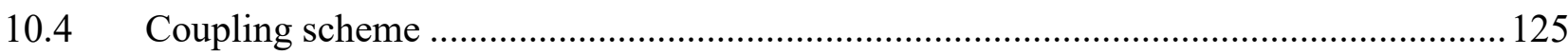

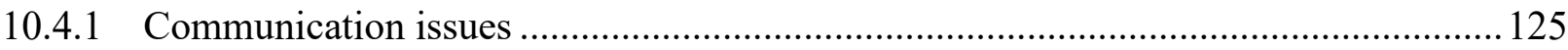

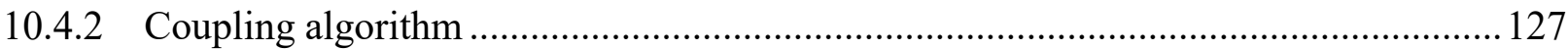

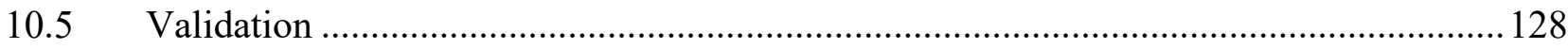

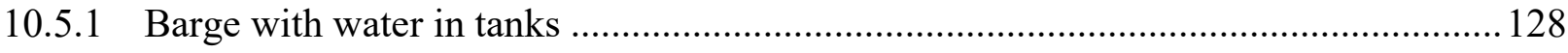

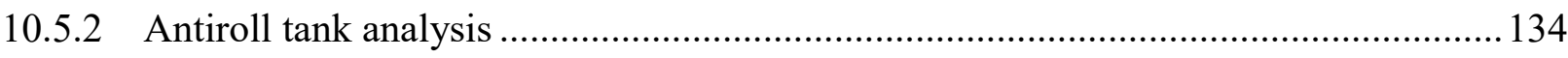

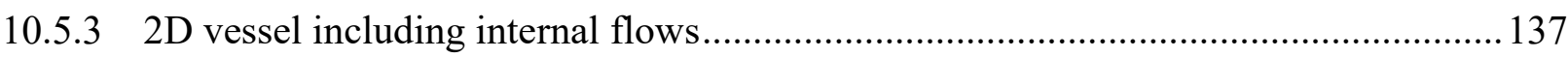

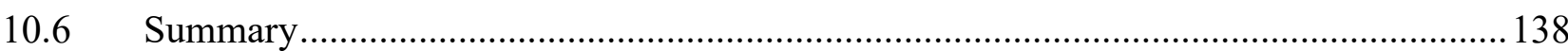

CHAPTER 11. ANALYSIS OF FLOATING WIND TURBINES RESPONSE ........................................ 141

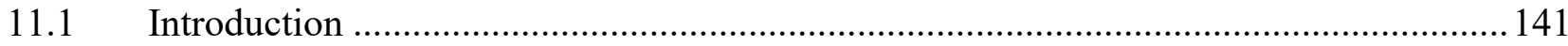

11.2 Validation of coupled seakeeping-mooring solver .................................................. 142

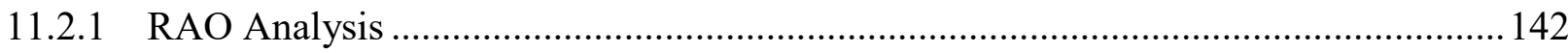

11.2.2 Mooring analysis around pitch resonance......................................................... 143

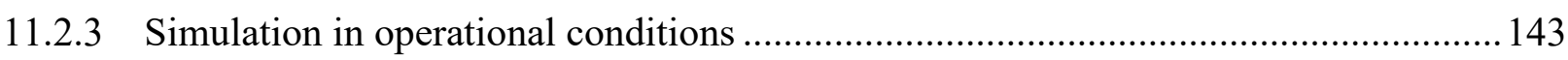

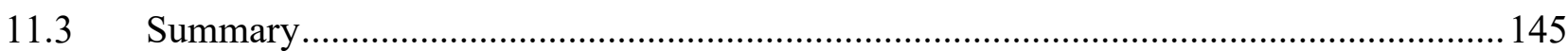

CHAPTER 12. ANALYSIS OF AN OSCILLATING WATER COLUMN DEVICE ................................. 149

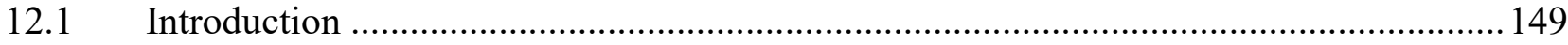

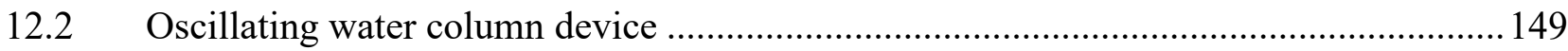

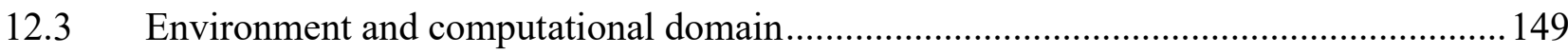




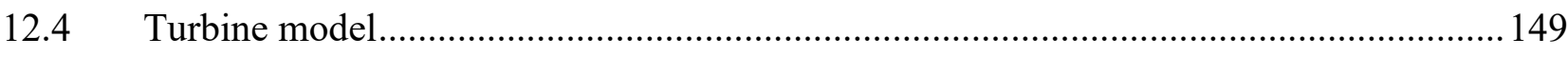

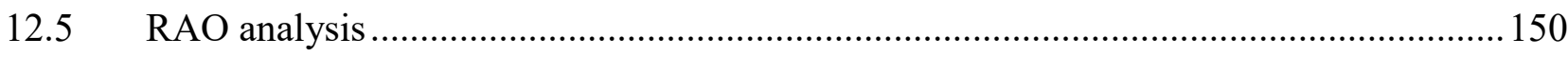

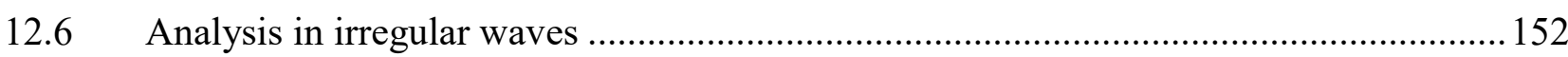

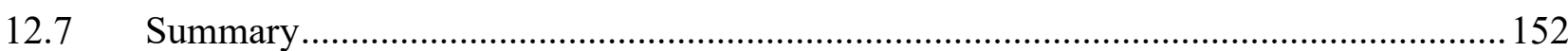

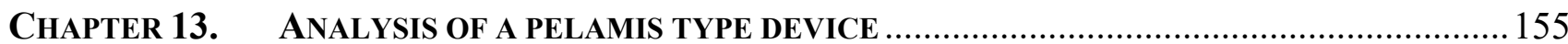

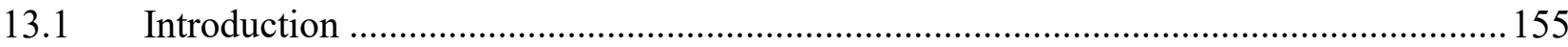

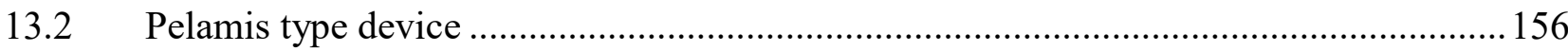

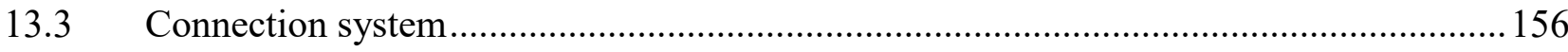

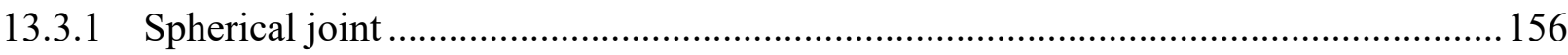

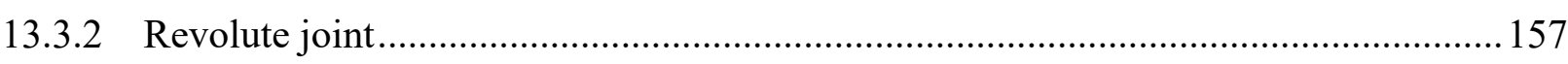

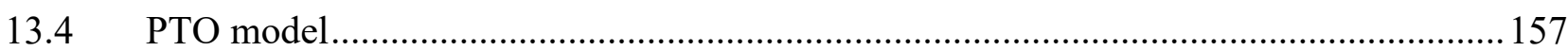

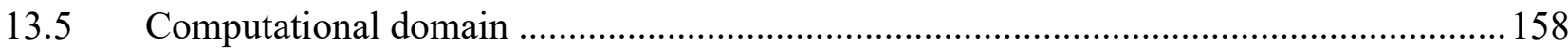

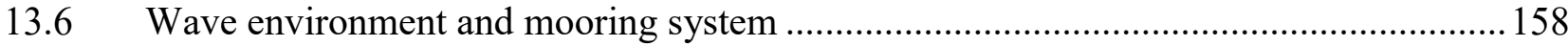

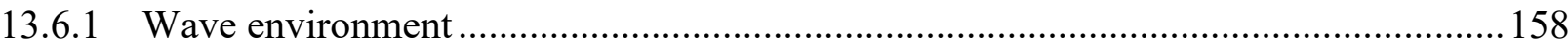

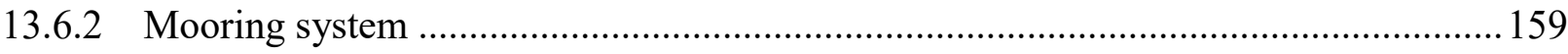

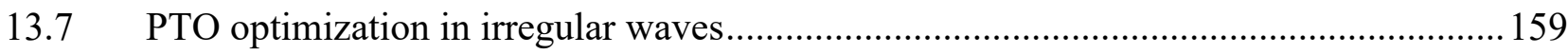

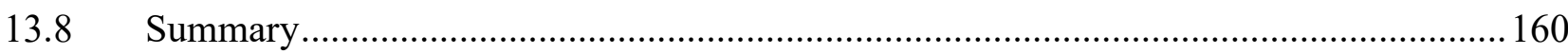

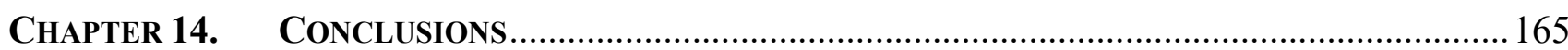

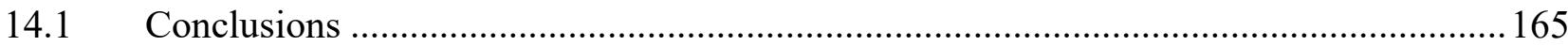

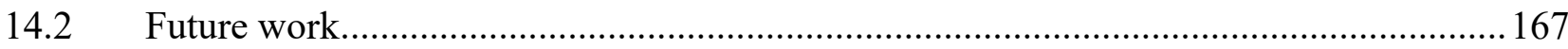

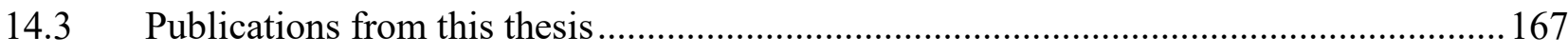

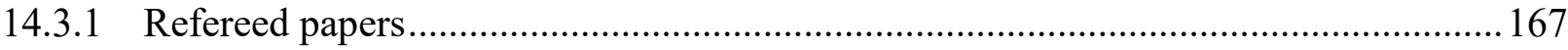

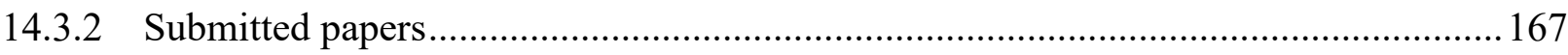

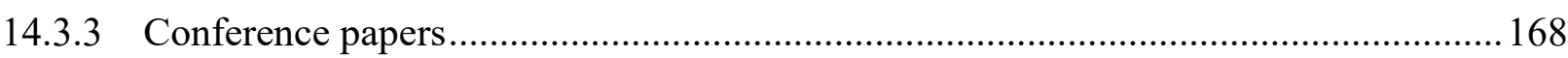

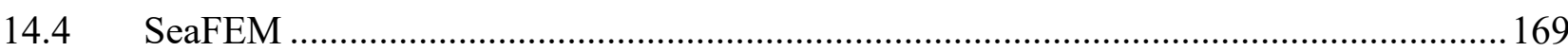

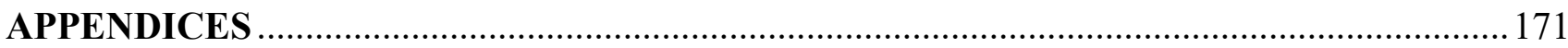

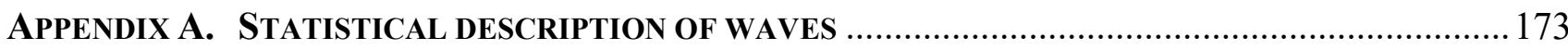

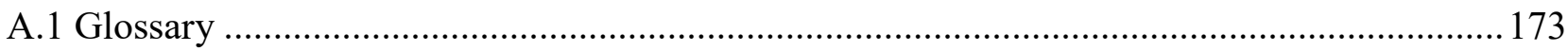

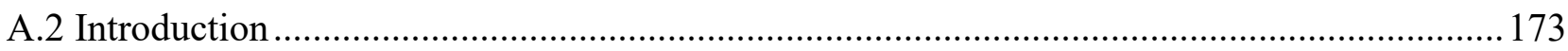

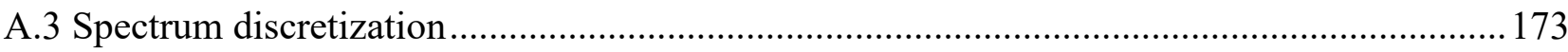

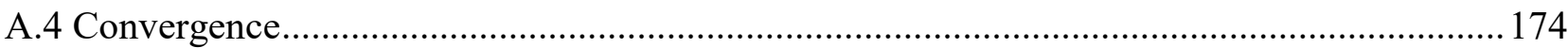

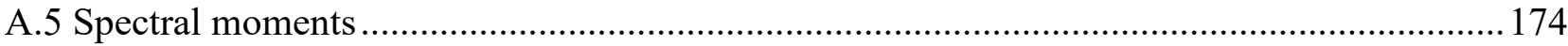

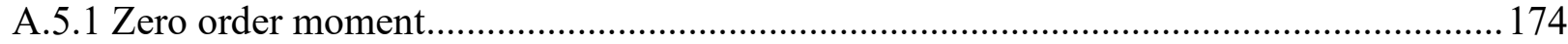

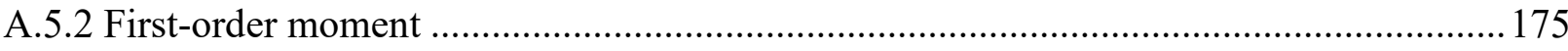

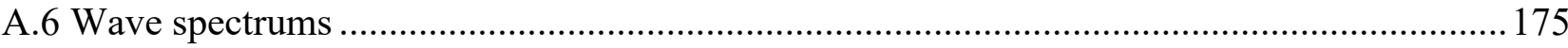

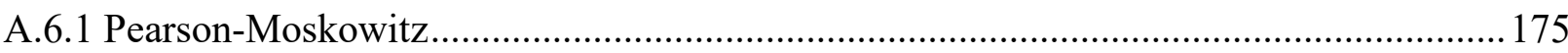




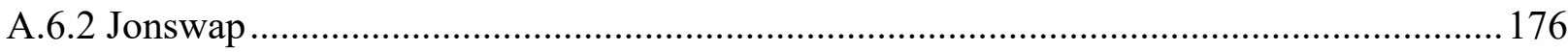

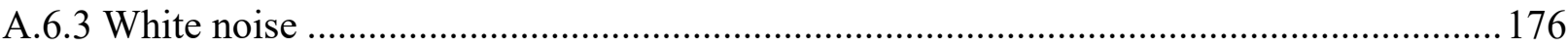

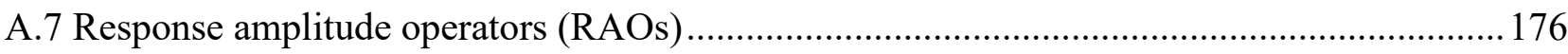

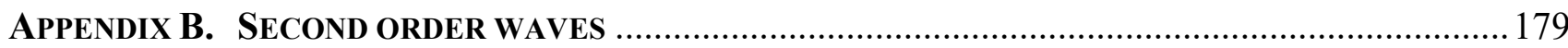

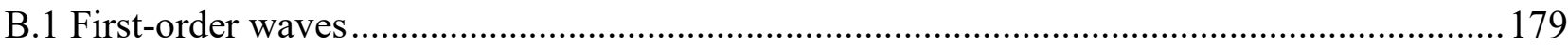

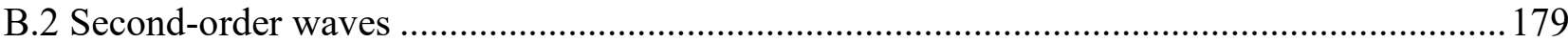

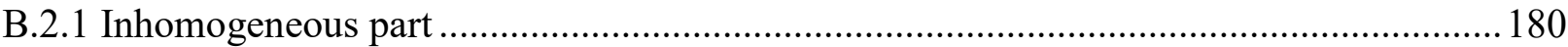

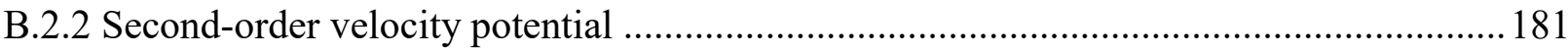

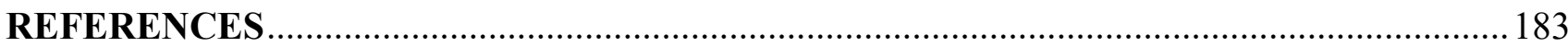




\section{A TIME-DOMAIN FINITE ELEMENT METHOD FOR SEAKEEPING AND WAVE RESISTANCE PROBLEMS}


The objective of this thesis is the research on numerical algorithms to develop numerical tools to simulate seakeeping problems as well as wave resistance problems of ships and floating structures.

The first tool developed is a wave diffraction-radiation solver. It is based on the finite element method (FEM) in order to solve the Laplace equation, as well as numerical schemes based on FEM, streamline integration, and finite difference method tailored for solving the free surface boundary condition.

It has been developed numerical tools to solve solid body dynamics of multibody systems with body links across them. This tool has been integrated with the wave diffraction-radiation solver to solve wave-body interaction problems.

Also it has been tailored coupling algorithms with other numerical tools in order to solve multiphysics problems. In particular, it has been performed coupling with a MEF structural solver to solve fluid-structure interaction problems, with a mooring solver, and with a solver capable of simulating internal flows in tanks to solve couple seakeeping-sloshing problems.

Numerical simulations have been carried out to validate and verify the developed algorithms, as well as to analyze case studies in the areas of marine engineering, offshore engineering, and offshore renewable energy. 
El objetivo de la tesis es la investigación de algoritmos numéricos para el desarrollo de herramientas numéricas para la simulación de problemas tanto de comportamiento en la mar como de resistencia al avance de buques y estructuras flotantes.

La primera herramienta desarrollada resuelve el problema de difracción y radiación de olas. Se basan en el método de los elementos finitos (MEF) para la resolución de la ecuación de Laplace, así como en esquemas basados en MEF, integración a lo largo de líneas de corriente, y en diferencias finitas desarrollados para la condición de superficie libre.

Se han desarrollado herramientas numéricas para la resolución de la dinámica de sólido rígido en sistemas multicuerpos con ligaduras. Estas herramientas han sido integradas junto con la herramienta de resolución de olas difractadas y radiadas para la resolución de problemas de interacción de cuerpos con olas.

También se han diseñado algoritmos de acoplamientos con otras herramientas numéricas para la resolución de problemas multifísica. En particular, se han realizado acoplamientos con una herramienta numérica basada de cálculo de estructuras con MEF para problemas de interacción fluido-estructura, otra de cálculo de líneas de fondeo, y con una herramienta numérica de cálculo de flujos en tanques internos para problemas acoplados de comportamiento en la mar con "sloshing".

Se han realizado simulaciones numéricas para la validación y verificación de los algoritmos desarrollados, así como para el análisis de diferentes casos de estudio con aplicaciones diversas en los campos de la ingeniería naval, oceánica, y energías renovables marinas. 


\section{Chapter 1. INTRODUCTION}

\subsection{Numerical simulation of seakeeping problems}

Seakeeping is a topic of great interest in marine and offshore engineering. The assessment of safety of ships and any device operating in rough seas is still a challenge. Moreover, this interest is growing in the last years due to the boost given by the development of marine renewable energies. In this context, the development of time domain seakeeping programs is a main request from the industry. Moreover, the simulation of multi-body systems is a key point in the development of more efficient marine renewable energy technologies, such as wave energy converters and floating wind turbines among others.

Up to date the numerical simulation of seakeeping problems has been mostly carried out in the frequency domain. The reason might be that computational cost of time-domain simulations was too high when compared to those of frequency-domain. Moreover, assumptions like linear waves and the harmonic nature of water waves made the frequency-domain to be the right choice. However, nowadays computing capabilities make possible to carry out numerical simulations in the time domain in a reasonable time. Time-domain has the advantage of simulating phenomena that cannot be handled in the frequency domain such as parametric resonance and other nonlinear effects. Furthermore, in the frequency domain, the simulation of multi-body systems requires calculating the interaction among the bodies, which increases quickly the computational effort as the number of bodies increase, and requires of acceleration techniques like the fast multipole technique (see Borgarino et. al [1]). On the other hand, when simulating in the time domain, the interaction among bodies is solved in a natural way without leading to a big increase of computational effort. Nowadays, more works are being carried out in the time domain (see Watai et. al [2] and Watai [3]).

Regarding the numerical method traditionally adopted for seakeeping simulations, the boundary element method (BEM) has dominated over others like finite element method (FEM). We might find the reason in the fact that most of the computational effort is spent in solving the Laplace equation. Then it might look like BEM offers a lower computational effort since it does not require to discretize the whole fluid volume. However, Cai et al. [4] carried out a heuristic study regarding the computational effort required for solving the Laplace equation by BEM and FEM. This study concluded that for a similar three-dimensional problem and a discretization size, the number of unknowns of BEM and FEM are $\mathrm{O}\left(\mathrm{N}^{2}\right)$ and $\mathrm{O}\left(\mathrm{N}^{3}\right)$, respectively, being $\mathrm{N}$ the number of unknowns needed in one dimension to achieve the desired discretization. But the computational costs are $\mathrm{O}\left(\mathrm{N}^{4}\right)$ 
and $\mathrm{O}\left(\mathrm{N}^{3}\right)$ respectively. Therefore, while BEM might be more efficient for problems with low number of unknowns, FEM becomes more efficient respect to BEM as the number of unknowns $\mathrm{N}$ increases. Considering that computational capabilities continuously increases, so does the complexity of problems to be undertaken, and the number of unknowns required. Hence FEM might become more efficient than BEM as the number of unknowns gets larger. Other than BEM and FEM, the finite difference or high order spectral methods have been also used to solve non-linear free surface flows. For instance, Ducrozet et. al [5] carried out a comparison study on the latter methods to solve ocean waves propagation.

Another advantage of FEM is the fact that it has been conceived for naturally solving the Laplace equation on unstructured meshes. This makes easier the discretization of complex computational domains.

In the last decade, there have been extensive applications of the finite element method (FEM) to free surface problems. For example, Oñate and García [6] presented a stabilized FEM for fluid structure interaction in the presence of free surface where the latter was modeled by solving a fictitious elastic problem on the moving mesh. In [7,8], Löhner et al. developed a FEM capable of tracking violent free surface flows interacting with objects. Also García et al. [9] developed a new technique to track complex free surface shapes. However, many works like the previous ones are based on solving the Navier-Stokes equations, too expensive computationally speaking when it comes to simulating real problems regarding ocean waves interacting with floating structures. These sorts of problems can be more cheaply simulated using potential flow along with Stokes' wave theory. For details on Stokes' wave theory, the reader is referred to [10].

With regards to wave-body interaction problems, there has been extensive work as well in the last decade. In [11], Wu and Eatock Taylor used both the FEM and the mixed FEM to analyze the twodimensional (2D) nonlinear transient water wave problems. Later $\mathrm{Wu}$ and Eatock Taylor [12] made a detailed comparison between FEM and the boundary element method (BEM) for the nonlinear free surface flow problem and found that the former was more efficient in terms of both CPU and memory requirement. Greaves et al. [13] employed quad-tree-based unstructured meshes to model fully nonlinear waves in 2D, using an ALE formulation in structured meshes. In [14] a spectral technique was adopted to simulate the 2D free surface flow problem. In [15] and [16], an implementation of FEM schemes to simulate 3D wave-body interaction was introduced using moving meshes along with an explicit time marching scheme for the free surface boundary condition. However, in those cases, remeshing and interpolation were needed, which leads to a high CPU cost. Westhuis [17] in his PhD dissertation developed a FEM code for nonlinear waves and focused in the development of groups of waves. The code relied in some specific structured mesh configurations and did not considered wave- 
body interaction. Hu et al. [18] applied FEM to study the case of a vertical cylinder under forced motions based on the works [11] and [12]. Turnbull et al. [19] coupled structured and unstructured meshes to simulate 2D wave-body interactions, and the estimation of vertical velocities at the free surface nodes required a prescribed number of nodes to be vertically aligned. Wu et al. [20] solved a $3 \mathrm{D}$ problem using a semi-structured mesh in the vertical direction. This way the nodes will be aligned vertically and the vertical component of the velocity at the free surface can be easily estimated by finite difference. Wang et al. [21] used FEM to study the effect of second-order wave sloshing within a tank in 2D. The fourth-order Runge-Kutta method was used as a time marching scheme for the free-surface boundary condition. A FEM solver for a second-order wave diffraction by an array of vertical cylinder using semi-structured mesh has been presented in [22]. Again, in order to estimate vertical velocity at the free surface nodes it is required a prescribed number of nodes to be aligned vertically. An explicit fourth-order Adams-Bashforth scheme was used as a time marching scheme for the free surface boundary condition. Later on, the same authors in [23] used a structured mesh based on rectangular elements to study second-order resonance effects. Yan et al. [24] applied the fully nonlinear potential for modeling overturning waves. To achieve that, a moving mesh technique was adopted to track down the free surface. Consequently, computational times are large. Recently, Song et al. developed a new Scaled Boundary Finite Element Method (SBFEM) for linear waves and structure interaction [25]. The SBFEM works in the frequency domain, and base functions for boundary elements based on Hankel functions were used for unbounded sub-domains where waves asymptotically disappear. This leads to a decrease in the number of elements needed for the simulation which improves the numerical efficiency of the method.

Despite of the great effort invested in the last years to the development of FEM algorithms, to the authors' knowledge, yet it has not been developed a fast FEM for solving first-order wave structure interaction in the time domain using unstructured meshes. In this thesis it is presented a FEM for wavestructure interaction that can be used with unstructured meshes. Besides, since it is based on Stokes' free surface approximation, no re-meshing or moving mesh technique are needed, which keeps computational costs and computational times lower. The developed algorithm has been designed to handle nonlinear external forces of any nature (mooring, internal flows, etc.).

\subsection{Numerical simulation of wave resistance problems}

Wave resistance estimation is a key point in the design of ships, offshore structures, and in recent years also in the design of devices aiming at harvesting marine renewable energies. With the increase of computational capability, computational fluid dynamics (CFD) tools have gained popularity in order to estimate the wave resistance. However, model testing in towing tanks is still a common practice. 
The main reason is that computing all aspects of the flow, such as wave making, vortex formation, boundary layers, and many other, is still very expensive computationally speaking, and require of expensive computers and large amounts of times.

When estimating the ship resistance, a common practice is to assume that the total resistance can be divided in two: viscous and non-viscous resistance. While the viscous resistance is related to the effects of boundary layers, vortex formation, and turbulence, the non-viscous resistance is related to wave formation and interaction with the ship. And while accurate CFD simulation of the former ones require of extremely low time steps and mesh sizes, the simulation of the latter can be achieved with quite good accuracy using potential flow theory, which is computationally much cheaper in comparison with computing the viscous resistance.

Looking at the non-viscous component, we can further decompose it into two main components: wave making resistance, and added resistance in waves. The first one is due to the wave formation when a body advances in still water, and is dominant component. The second one is the increase of resistance when the ship advance in waves respect to still water, and this is a second-order component compared to the wave making resistance (except for very low Froude numbers).

The added resistance is an increase of resistance happening in the presence of waves. This resistance has three main components: the first due to wave diffraction; the second due to wave radiation (induced mainly by heave and pitch motions); and the third due to variations of the wetted surface. As a result, the average resistance can be increased in the order of $40 \%$ for some specific wave frequencies where resonance occurs.

A common practice is to estimate viscous resistance by a simplified formulation based on a friction line for a flat surface (such as ITTC-57, Schoenherr) depending on the Reynolds number and on a constant form factor coefficient. Then subtracting the viscous component to the total resistance (obtained by model testing) gives the wave making component (residual resistance). This approximation assumes that the form factor is the same at any Froude number. However, estimations of the form factors of the same hull at different model basins show a large dispersion, and therefore experimental determination of wave making resistance may results in significant errors [26].

Wave making and added resistance cannot be approximated so easily by simplified formulae as the viscous resistance, and CFD analyses are becoming an useful tool for such a task. Probably the first computer method for solving steady-state wave making resistance was introduced by Dawson in [27]. Dawson used the Boundary Element Method (BEM), also called Rankine panel method, in combination with upstream finite difference schemes for the convective term of the free surface. Moreover, the free surface boundary conditions were expanded in Taylor series around the mean water level to simplify their imposition. It can be said that Dawson stablished the basis of the current methods 
for estimating wave making resistance using potential flow. For instance in $[28,29]$ the Dawson method's is described and further analysis regarding linearizations and nonlinear terms are carried out.

Despite of the importance of the added resistance in waves and its impact on the long term performance of a ship, there are not many works in the literature coping with this problem from a CFD point of view. Some formulations have been derived by different authors as an extension of the mean drift force induced by waves in the presence of a water current [30]. These approaches basically modify damping and added mass coefficients, as well as the mean drift forces, to account for the current effect. However, convective terms in the free surface boundary conditions are neglected, limiting this approach to low Froude numbers. A more sophisticated approach is to solve the three dimensional potential flow problem in the frequency domain (usually using BEM), and then obtain the added resistance using Maruo's approach [31,32,33]. Maruo's approach still does not account for the convective terms in the free surface boundary conditions, and therefore is also limited to low Froude numbers. Finally, few works can be found trying to solve the added resistance in waves in the time domain, considering the convective terms in the free surface boundary condition, which overcome the limitation of low Froude numbers [34].

Most of the computer methods used for estimating the wave resistance are based on expanding the free surface boundary conditions around the mean water level, and using a perturbation method in order to retain the leading order terms. This approach has the advantage of imposing the free surface conditions on a steady flat surface, rather than having to track the free surface and modify the computational domain accordingly. While the latter approach could solve the fully nonlinear free surface conditions, the former is commonly preferred because it reduces greatly the computational effort while providing good accuracy for the wave making resistance problem.

Up to date, computer methods for the wave resistance problem, as described above, are based on BEM (see for instance [35,36]), and their algorithms require the use of structured meshes on the free surface. While these methods are widely and successfully used, the use of structured meshes can be a limitation in some cases.

In this thesis, a computer method for estimating the wave making and added resistance is proposed. The method is based on potential flow with approximation of the boundary conditions on the mean water level for computational efficiency, as is commonly used. The main novelties are the use of the Finite Element Method (FEM), rather than (BEM), and the use of two numerical schemes that allow the use of unstructured meshes at the free surface.

Nowadays, potential flow solvers offer the possibility of computing the wave making and added resistance in an affordable manner, economically and computationally speaking, which results cheaper and faster than model testing. Hence the author is interested in developing a new computer method to 
handle this sort of problems that can be efficiently used in the design of a ship, as well as in offshore structures by quantifying the effect of water currents.

\subsection{Outline of this thesis}

This thesis is organized in three main parts. Each one contents a set of consecutive chapters focusing in three specific aspects: mathematical-numerical basis, verification-validation, and application.

The first block extends from Chapter 2 to 0 and presents the theory of the different solvers developed in this thesis. Within each chapter, and introduction to the mathematical model is given, presenting the governing equations and assumptions made. Then, the numerical schemes used for solving the governing equations are given in detail, as well as the implementation algorithms.

The second block extends from Chapter 5 to Chapter 8 and presents verification and validation cases to assess the validity of the developed solvers. It also looks into the computing performance, presenting and analyzing some solver acceleration techniques.

The third block extends from Chapter 9 to Chapter 13. In each chapter, an analysis is carried out for a specific application example. The main objective of this block is to provide an idea about the wide range of application of the developed solvers. 
PART I: MATHEMATICAL AND

NUMERICAL MODELS 


\section{Chapter 2. SEAKEEPING PROBLEMS}

\subsection{Mathematical model for seakeeping problems}

\subsubsection{Glossary}

$v \quad$ Fluid velocity field

$\boldsymbol{v}_{\varphi} \quad$ Fluid velocity

$\varphi \quad$ Velocity potential

$\xi \quad$ Free surface elevation

$\boldsymbol{v}_{\psi} \quad$ Incident wave induced velocity

$\psi \quad$ Incident velocity potential

$\zeta$ Incident wave elevation

$\boldsymbol{v}_{\phi} \quad$ Diffraction-radiation wave induced velocity

$\phi \quad$ Diffraction-radiation velocity potential

$\eta \quad$ Diffraction-radiation wave elevation

$P_{f s} \quad$ Free surface pressure

$P_{p h}^{0} \quad$ Hydrostatic pressure at point $P$.

$P_{p h}^{1} \quad$ First-order hydrostatic pressure variation at point $\mathrm{P} \in \mathrm{S}_{B}$.

$P_{p h}^{1+2} \mathrm{Up}$ to second-order hydrostatic pressure variation at point $\mathrm{P} \in \mathrm{S}_{B}$.

$P_{\psi p}^{1} \quad$ First-order pressure at point $\mathrm{P}$ induced by INCIDENT wave

$P_{\psi p}^{1+2} \quad$ Up to second-order Pressure at point $\mathrm{P}$ induced by INCIDENT wave

$P_{\phi p}^{1} \quad$ First-order pressure at point $\mathrm{P}$ induced by DIF-RAD wave

$P_{\phi p}^{1+2} \quad$ Up to second-order Pressure at point $\mathrm{P}$ induced by DIF-RAD wave

$\boldsymbol{r}_{p}^{1} \quad$ First-order body surface displacement at point $\mathrm{P}$

$\boldsymbol{r}_{p}^{1+2} \quad$ Up to second-order body surface displacement at point $\mathrm{P}$

$\boldsymbol{n}_{p}^{0} \quad$ Initial body surface normal vector at point $\mathrm{P}$

$\boldsymbol{n}_{p}^{1} \quad$ Body surface normal vector after first-order movement at point $\mathrm{P}$

$\boldsymbol{R}^{0} \quad$ Origin of coordinates of body initial reference frame

$\boldsymbol{P}^{0} \quad$ Point on initial body surface

$\boldsymbol{H}$ Second-order transformation matrix

$v_{p} \quad$ Body velocity over body surface at point $\mathrm{P}$

$\boldsymbol{v}_{p}^{1} \quad$ First-order body velocity over body surface at point $\mathrm{P}$

$\boldsymbol{v}_{p}^{1+2} \quad$ Up to second-order body velocity over body surface at point $\mathrm{P}$

$\Omega \quad$ Fluid domain

$\mathrm{S}_{B} \quad$ Instantaneous body boundary 
$\mathrm{S}_{B}^{0} \quad$ Initial body boundary

$\mathrm{S}_{B}^{1} \quad$ Instantaneous body boundary corresponding to first-order movements

$\mathrm{S}_{B}^{1+2}$ Instantaneous body boundary corresponding to up to second-order movements

$S_{S} \quad$ Seabed boundary

A Wave amplitude

$\omega \quad$ Wave angular frequency

$K \quad$ Wave number

$L \quad$ Wave length

T Wave period

$\gamma \quad$ Wave direction respect to $\mathrm{OX}$ axis

$\delta_{i} \quad$ Wave phase delay

$H \quad$ Water depth

\subsubsection{Introduction}

Section 2.1 presents the governing equations, based on potential flow and incompressible fluid, of the wave problem. First, the general governing equations are presented. Second, the traditional perturbation solution approach is introduced. And third, first and second-order governing equations for the wave problem are derived.

\subsubsection{Governing equations}

\subsubsection{Flow equation and boundary conditions}

Assuming incompressible flow $(\nabla \cdot \mathbf{v}=0)$ and irrotational flow $(\nabla \times \mathbf{v}=0 \Rightarrow \mathbf{v}=\nabla \varphi)$, then, the flow governing equations are given by:

$$
\begin{aligned}
& \Delta \varphi=0 \quad \text { in } \Omega \quad \text { incompressible and irrotational flow, } \\
& \frac{\partial \xi}{\partial t}+\frac{\partial \varphi}{\partial x} \frac{\partial \xi}{\partial x}+\frac{\partial \varphi}{\partial y} \frac{\partial \xi}{\partial y}-\frac{\partial \varphi}{\partial z}=0 \quad \text { on } z=\xi \quad \text { free surface kinematic boundary } \\
& \frac{\partial \zeta}{\partial t}+\frac{\partial \varphi}{\partial \mathrm{x}} \frac{\partial \zeta}{\partial x}+\frac{\partial \varphi}{\partial \mathrm{y}} \frac{\partial \xi}{\partial y}-\frac{\partial \varphi}{\partial \mathrm{z}}=0 \quad \text { on } z=\xi \quad \text { condition, } \\
& \frac{\partial \varphi}{\partial \mathrm{t}}+\frac{1}{2} \nabla \varphi \cdot \nabla \varphi+\frac{P_{f s}}{\rho}+g \xi=0 \quad \text { on } z=\xi \quad \text { free surface dynamic boundary condition, } \\
& \boldsymbol{v}_{p} \cdot \boldsymbol{n}_{p}+\boldsymbol{v}_{\varphi} \cdot \boldsymbol{n}_{p}=0 \quad \text { on } \mathrm{P} \in \mathrm{S}_{B} \quad \text { body boundary condition, } \\
& \boldsymbol{v}_{\varphi} \cdot \boldsymbol{n}_{p}=0 \quad \text { on } \mathrm{P} \in \mathrm{S}_{S} \quad \text { seabed boundary condition, }
\end{aligned}
$$

and the pressure at any given point $\mathrm{P}$ is:

$$
P_{p}=-\rho \frac{\partial \varphi}{\partial t}-\frac{1}{2} \rho \nabla \varphi \cdot \nabla \varphi-\rho g z_{p} \quad \text { pressure at a point } \mathrm{P} .
$$




\subsubsection{Solution procedure}

\section{Taylor series expansion}

Free surface and body boundary conditions (BCs) will be applied on $z=0$ and $\mathrm{S}_{B}^{0}$ respectively. Then, Taylor series expansion is carried out to both BCs around $z=0$ and $\mathrm{S}_{B}^{0}$ to approximate the BC on $z=\xi$ and $S_{B}$ respectively.

\section{Perturbed solution}

A perturbed solution based on Stokes' waves approximation is used, where the velocity potential and free surface elevation are perturbed as (see for instance Newman 1977, chapter 6 [37]):

$$
\begin{aligned}
& \varphi=\epsilon^{1} \varphi^{1}+\epsilon^{2} \varphi^{2}+\epsilon^{3} \varphi^{3}+\cdots, \\
& \xi=\epsilon^{1} \xi^{1}+\epsilon^{2} \xi^{2}+\epsilon^{3} \xi^{3}+\cdots .
\end{aligned}
$$

Body movement solution is also assumed to be a perturbed solution:

$$
\begin{aligned}
& \boldsymbol{X}=\epsilon^{1} \boldsymbol{X}^{1}+\epsilon^{2} \boldsymbol{X}^{2}+\epsilon^{3} \boldsymbol{X}^{3} \ldots \\
& \boldsymbol{V}=\epsilon^{1} \boldsymbol{V}^{1}+\epsilon^{2} \boldsymbol{V}^{2}+\epsilon^{3} \boldsymbol{V}^{3} \ldots
\end{aligned}
$$

Then the translational vector of any point $\mathrm{P}$ on the body surface can be perturbed as:

$\boldsymbol{r}_{p}=\epsilon^{1} \boldsymbol{r}_{p}^{1}+\epsilon^{2} \boldsymbol{r}_{p}^{2}+\epsilon^{3} \boldsymbol{r}_{p}^{3} \ldots$

The linear and angular displacements of a point $\mathbf{P}^{0} \in S_{B}^{0}$ are given by (see Figure 1):

$$
\begin{aligned}
& \boldsymbol{X}^{i}=\left(\delta_{x}^{i}, \delta_{y}^{i}, \delta_{z}^{i}, \theta_{x}^{i}, \theta_{y}^{i}, \theta_{z}^{i}\right)=\left(\boldsymbol{\delta}^{i}, \boldsymbol{\theta}^{i}\right), \\
& \boldsymbol{r}_{p}^{1}=\boldsymbol{\delta}^{1}+\boldsymbol{\theta}^{1} \times \overrightarrow{\boldsymbol{R}^{0} \boldsymbol{P}^{0}} \\
& \boldsymbol{r}_{p}^{1+2}=\boldsymbol{\delta}^{1+2}+\boldsymbol{\theta}^{1+2} \times \overrightarrow{\boldsymbol{R}^{0} \boldsymbol{P}^{0}}+\overline{\overline{\boldsymbol{H}}} \overrightarrow{\boldsymbol{R}^{0} \boldsymbol{P}^{0}} \\
& \overline{\overline{\boldsymbol{H}}}=\frac{1}{2}\left(\begin{array}{ccc}
-\left(\theta_{y}^{2}+\theta_{z}^{2}\right) & 0 & 0 \\
2 \theta_{x} \theta_{y} & -\left(\theta_{x}^{2}+\theta_{z}^{2}\right) & 0 \\
2 \theta_{x} \theta_{z} & 2 \theta_{y} \theta_{z} & -\left(\theta_{x}^{2}+\theta_{y}^{2}\right)
\end{array}\right) \text {, } \\
& \boldsymbol{V}^{i}=\left(v_{x}^{i}, v_{y}^{i}, v_{z}^{i}, \omega_{x}^{i}, \omega_{y}^{i}, \omega_{z}^{i}\right)=\left(\boldsymbol{v}^{i}, \boldsymbol{\omega}^{i}\right) \text {, } \\
& \boldsymbol{v}_{p}^{1}=\boldsymbol{v}^{1}+\boldsymbol{\omega}^{1} \times \overrightarrow{\boldsymbol{R}^{0} \boldsymbol{P}^{0}} \\
& \boldsymbol{v}_{p}^{1+2}=\boldsymbol{v}^{1+2}+\boldsymbol{\omega}^{1+2} \times \overrightarrow{\boldsymbol{R}^{0} \boldsymbol{P}^{0}}+\dot{\overline{\boldsymbol{H}}} \overrightarrow{\boldsymbol{R}^{0} \boldsymbol{P}^{0}} \\
& \boldsymbol{n}_{p}^{1}=\boldsymbol{n}_{p}^{0}+\boldsymbol{\theta}^{1} \times \boldsymbol{n}_{p}^{0} .
\end{aligned}
$$

\subsubsection{First-order approach}

\subsubsection{First-order governing equations}

After carrying out the Taylor series expansions, introduced the perturbed solution, and retaining terms of order $\epsilon$, the first-order governing equations become:

$$
\Delta \varphi^{1}=0 \quad \text { in } \Omega,
$$


$\frac{\partial \xi^{1}}{\partial t}-\frac{\partial \varphi^{1}}{\partial \mathrm{z}}=0 \quad$ on $z=0$

$\frac{\partial \varphi^{1}}{\partial \mathrm{t}}+\frac{P_{f s}}{\rho}+g \xi^{1}=0 \quad$ on $z=0$,

$\boldsymbol{v}_{p}^{1} \cdot \boldsymbol{n}_{p}^{0}+\boldsymbol{v}_{\varphi}^{1} \cdot \boldsymbol{n}_{p}^{0}=0 \quad$ on $P \in S_{B}^{0}$,

$\boldsymbol{v}_{\varphi}^{1} \cdot \boldsymbol{n}_{\mathrm{p}}=0 \quad$ on $P \in S_{S}$

and the first-order pressure at a point $P_{p}^{1}$ on the body surface is

$P_{p}^{1}=P_{H}^{0}+P_{H}^{1}+P_{D}^{1}$

where $P_{D}^{1}=-\rho \frac{\partial \varphi^{1}}{\partial t}, P_{H}^{0}=-\rho g z_{p}$, and $P_{H}^{1}=-\rho g r_{p z}^{1}$.

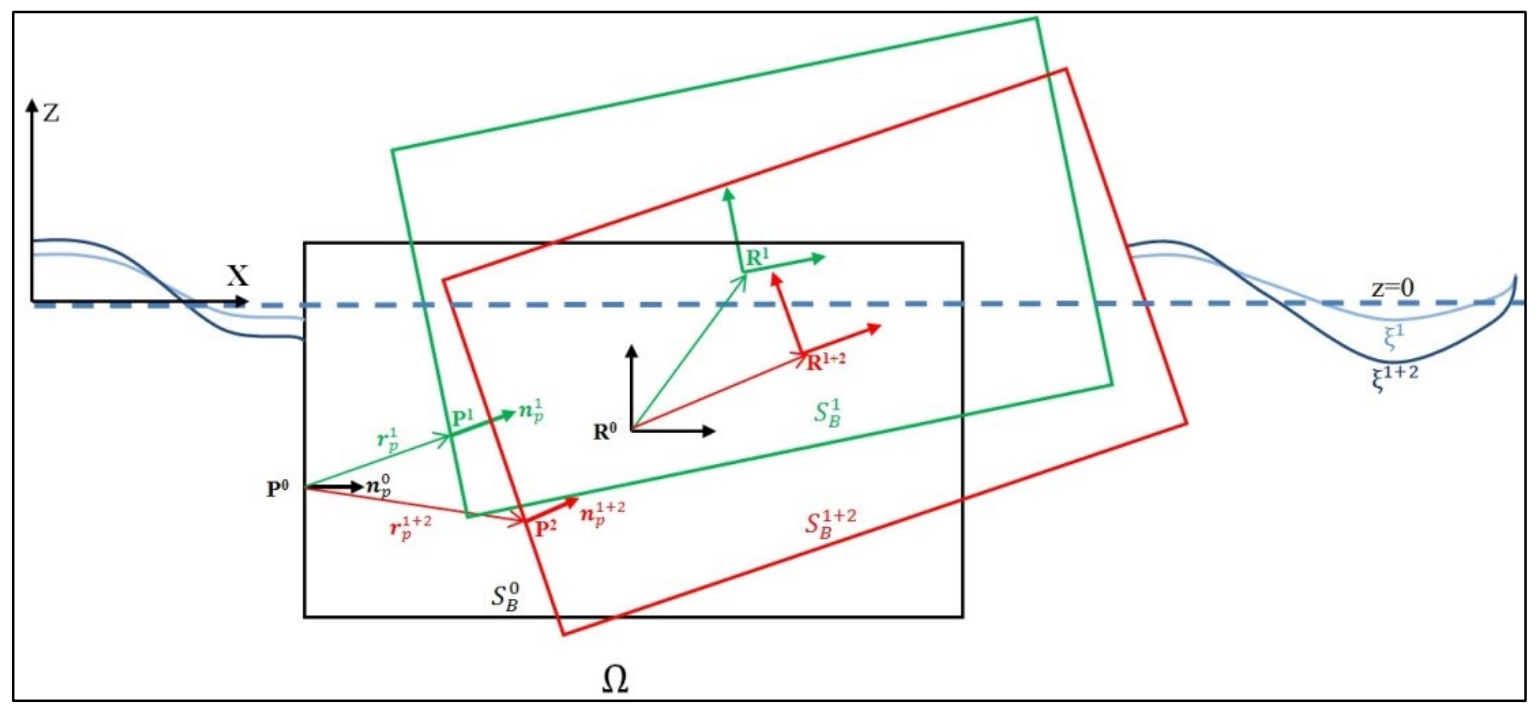

Figure 1: First and second-order rigid body movement.

\subsubsection{First-order decomposition solution}

The total velocity potential can be decomposed as:

$$
\begin{aligned}
& \varphi^{1}=\psi^{1}+\phi^{1}, \\
& \xi^{1}=\zeta^{1}+\eta^{1},
\end{aligned}
$$

where $\psi^{1}$ and $\zeta^{1}$ are the incident wave velocity potential and free surface elevation respectively, and $\phi^{1}$ and $\eta^{1}$ are the diffraction-radiation wave velocity potential and free surface elevation respectively.

\section{First-order incident wave solution}

The incident wave velocity potential $\psi^{1}$ fulfils the following equations:

$$
\begin{array}{ll}
\Delta \psi^{1}=0 & \text { in } \Omega, \\
\frac{\partial \zeta^{1}}{\partial t}-\frac{\partial \psi^{1}}{\partial \mathrm{z}}=0 & \text { on } z=0, \\
\frac{\partial \psi^{1}}{\partial \mathrm{t}}+g \zeta^{1}=0 & \text { on } z=0
\end{array}
$$


$\frac{\partial \psi^{1}}{\partial \mathrm{z}}=0 \quad$ on $z=-H$

Eqs. (2-28)-(2-30) has an analytical solution, given by the Airy's wave theory [37]:

$$
\begin{aligned}
& \psi^{1}=\sum_{i} \frac{A_{i} g}{\omega_{i}} \frac{\cosh \left(\left|\boldsymbol{k}_{i}\right|(H+z)\right)}{\cosh \left(\left|\boldsymbol{k}_{i}\right| H\right)} \sin \left(\boldsymbol{k}_{i} \boldsymbol{x}-\omega_{i} t+\delta_{i}\right), \\
& \zeta^{1}=\sum_{i} A_{i} \cos \left(\boldsymbol{k}_{i} \boldsymbol{x}-\omega_{i} t+\delta_{i}\right),
\end{aligned}
$$

where $\boldsymbol{k}=\frac{2 \pi}{L}(\cos (\gamma), \sin (\gamma))$. The following dispersion relation holds:

$$
\omega_{i}^{2}=g\left|\boldsymbol{k}_{i}\right| \tanh \left(\left|\boldsymbol{k}_{i}\right| H\right),
$$

and the fluid pressure induced at a point $\mathrm{P}$ is given by:

$$
P_{p \psi}^{1}=\sum_{i} \rho g A_{i} \frac{\cosh \left(\left|\boldsymbol{k}_{i}\right|(H+z)\right)}{\cosh \left(\left|\boldsymbol{k}_{i}\right| H\right)} \cos \left(\boldsymbol{k}_{i} \boldsymbol{x}-\omega_{i} t+\delta_{i}\right) \text {. }
$$

In the asymptotic case of infinite water depth $H \rightarrow \infty$ and the factor $\cosh (|\boldsymbol{k}|(H+z)) /$ $\cosh (|\boldsymbol{k}| H) \rightarrow \exp (|\boldsymbol{k}| z)$.

\section{First-order diffraction-radiation wave problem}

The governing equations for the diffraction-radiation velocity potential $\phi^{1}$ si given by [37]:

$$
\begin{array}{ll}
\Delta \phi^{1}=0 & \text { in } \Omega, \\
\frac{\partial \eta^{1}}{\partial t}-\frac{\partial \phi^{1}}{\partial \mathrm{z}}=0 & \text { on } z=0, \\
\frac{\partial \phi^{1}}{\partial \mathrm{t}}+\frac{P_{f S}}{\rho}+g \eta^{1}=0 & \text { on } z=0, \\
\boldsymbol{v}_{\phi}^{1} \cdot \boldsymbol{n}_{p}^{0}=-\boldsymbol{v}_{p}^{1} \cdot \boldsymbol{n}_{p}^{0}-\boldsymbol{v}_{\psi}^{1} \cdot \boldsymbol{n}_{p}^{0} & \text { on } P \in S_{B}^{0}, \\
\boldsymbol{v}_{\phi}^{1} \cdot \boldsymbol{n}_{p}=-\boldsymbol{v}_{\psi}^{1} \cdot \boldsymbol{n}_{p} & \text { on } P \in S_{S},
\end{array}
$$

and the fluid pressure at a point $\mathrm{P}$ on the body surface is given by:

$$
P_{p}^{1}=P_{p h}^{0}+P_{p h}^{1}+P_{p \psi}^{1}+P_{p \phi}^{1},
$$

where $P_{p \phi}^{1}=-\rho \frac{\partial \phi^{1}}{\partial t}, P_{p h}^{0}=-\rho g z$, and $P_{p h}^{1}=-\rho g r_{p z}^{1}$.

\subsubsection{Second-order approach}

\subsubsection{Second-order governing equations}

After carrying out the Taylor series expansion, introducing the perturbed solution, and retaining terms up to second order $\epsilon^{2}$, and considering that $\varphi^{1+2}=\varphi^{1}+\varphi^{2}, \xi^{1+2}=\xi^{1}+\xi^{2}, P_{p}^{1+2}=P_{p}^{1}+P_{p}^{2}$, $X_{B}^{1+2}=X_{B}^{1}+X_{B}^{2}, \quad V_{B}^{1+2}=V_{B}^{1}+V_{B}^{2}, \quad v_{p}^{1+2}=v_{p}^{1}+v_{p}^{2}, \quad \boldsymbol{v}_{\varphi}^{1+2}=\boldsymbol{v}_{\varphi}^{1}+\boldsymbol{v}_{\varphi}^{2}, \quad \boldsymbol{r}_{p}^{1+2}=\boldsymbol{r}_{p}^{1}+\boldsymbol{r}_{p}^{2}, \quad$ the governing equations become:

$$
\Delta \varphi^{1+2}=0 \quad \text { in } \Omega,
$$




$$
\begin{array}{ll}
\frac{\partial \xi^{1+2}}{\partial t}-\frac{\partial \varphi^{1+2}}{\partial z}=\xi^{1} \frac{\partial}{\partial z}\left(\frac{\partial \varphi^{1}}{\partial z}\right)-\frac{\partial \xi^{1}}{\partial x} \frac{\partial \varphi^{1}}{\partial x}-\frac{\partial \xi^{1}}{\partial y} \frac{\partial \varphi^{1}}{\partial y} & \text { on } z=0 \\
\frac{\partial \varphi^{1+2}}{\partial t}+\frac{P_{f s}}{\rho}+g \xi^{1+2}=-\xi^{1} \frac{\partial}{\partial z}\left(\frac{\partial \varphi^{1}}{\partial t}\right)-\frac{1}{2} \nabla \varphi^{1} \cdot \nabla \varphi^{1} & \text { on } z=0 \\
\left(\boldsymbol{v}_{p}^{1}+\boldsymbol{v}_{\varphi}^{1}\right) \cdot \boldsymbol{n}_{p}^{1}+\left(\boldsymbol{v}_{p}^{2}+\boldsymbol{v}_{\varphi}^{2}\right) \cdot \boldsymbol{n}_{p}^{0}=-\left(\boldsymbol{r}_{p}^{1} \cdot \nabla\right) \boldsymbol{v}_{\varphi}^{1} \cdot \boldsymbol{n}_{p}^{0} & \text { on } P \in S_{B}^{0}, \\
\boldsymbol{v}_{\varphi}^{1+2} \cdot \boldsymbol{n}_{p}=0 & \text { on } P \in S_{S},
\end{array}
$$

and the pressure at a point $\mathrm{P}$ on the body surface is:

$$
P_{p}^{1+2}=P_{H}^{0}+P_{H}^{1+2}+P_{D}^{1+2}
$$

where $P_{H}^{0}=-\rho g z_{p}$, and $P_{H}^{1+2}=-\rho g r_{p Z}^{1+2}$, and $P_{D}^{1+2}=-\rho \frac{\partial \varphi^{1+2}}{\partial t}-\rho \boldsymbol{r}_{p}^{1} \cdot \nabla\left(\frac{\partial \varphi^{1}}{\partial t}\right)-\rho \frac{1}{2} \nabla \varphi^{1} \cdot \nabla \varphi^{1}$.

\subsubsection{Second-order decomposition solution}

\section{Second-order incident wave solution}

The second-order total velocity potential can be decomposed as:

$$
\begin{aligned}
& \varphi^{2}=\psi^{2}+\phi^{2}, \\
& \xi^{2}=\zeta^{2}+\eta^{2},
\end{aligned}
$$

where $\psi^{2}$ and $\zeta^{2}$ are the second-order incident wave velocity potential and free surface elevation, and $\phi^{2}$ and $\eta^{2}$ are the second-order diffraction-radiation wave velocity potential and free surface elevation. Up to second-order incident wave potential and free surface elevation fulfils the following equations:

$$
\begin{array}{ll}
\Delta \psi^{1+2}=0 & \text { in } \Omega, \\
\frac{\partial \zeta^{1+2}}{\partial t}-\frac{\partial \psi^{1+2}}{\partial \mathrm{z}}=\zeta^{1} \frac{\partial^{2} \psi^{1}}{\partial z^{2}}-\frac{\partial \zeta^{1}}{\partial x} \frac{\partial \psi^{1}}{\partial x}-\frac{\partial \zeta^{1}}{\partial y} \frac{\partial \psi^{1}}{\partial y} & \text { on } z=0, \\
\frac{\partial \psi^{1+2}}{\partial \mathrm{t}}+g \zeta^{1+2}=-\zeta^{1} \frac{\partial}{\partial z}\left(\frac{\partial \psi^{1}}{\partial t}\right)-\frac{1}{2} \nabla \psi^{1} \cdot \nabla \psi^{1} & \text { on } z=0, \\
\frac{\partial \psi^{1+2}}{\partial \mathrm{z}}=0 & \text { on } z=-H,
\end{array}
$$

The solution to Eqs.(2-50)-(2-53) is as follows (see Appendix B):

$$
\begin{aligned}
& \psi^{1+2}=\psi^{1}+\sum_{i} B_{i j}^{0} \sin \left(2\left(\boldsymbol{k}_{i} \boldsymbol{x}-\omega_{i} t+\delta_{i}\right)\right) \\
& +\sum_{j} \sum_{i} B_{i j}^{+} \cosh \left(\left|\boldsymbol{k}_{i}+\boldsymbol{k}_{j}\right|(H+z)\right) \sin \left(\left(\boldsymbol{k}_{i} \boldsymbol{x}-\omega_{i} t+\delta_{i}\right)+\left(\boldsymbol{k}_{j} \boldsymbol{x}-\omega_{j} t+\delta_{j}\right)\right) \\
& +\sum_{j} \sum_{i} B_{i j}^{-} \cosh \left(\left|\boldsymbol{k}_{i}-\boldsymbol{k}_{j}\right|(H+z)\right) \sin \left(\left(\boldsymbol{k}_{i} \boldsymbol{x}-\omega_{i} t+\delta_{i}\right)-\left(\boldsymbol{k}_{j} \boldsymbol{x}-\omega_{j} t+\delta_{j}\right)\right),
\end{aligned}
$$

where the coefficients are given in Table 1 . 
In the asymptotic case of infinite depth $(\mathrm{H} \rightarrow \infty)$ the coefficients $B_{i j}^{0} \rightarrow 0, D_{i j}^{+} \rightarrow \infty, D_{i j}^{-} \rightarrow \infty$, $B_{i j}^{+} \rightarrow 0, B_{i j}^{-} \rightarrow 0$. Then, the second-order velocity potential becomes null.

The wave elevation up to second order is obtained from:

$$
\zeta^{1+2}=-\frac{1}{g}\left(\frac{\partial \psi^{1+2}}{\partial \mathrm{t}}+\frac{P}{\rho}+\zeta^{1} \frac{\partial}{\partial z}\left(\frac{\partial \psi^{1}}{\partial t}\right)+\frac{1}{2} \nabla \psi^{1} \cdot \nabla \psi^{1}\right),
$$

and the fluid pressure induced by the second-order wave potential at a point $\mathrm{P}$ is:

$$
P_{p \psi}^{1+2}=-\rho\left(\frac{\partial \psi^{1+2}}{\partial \mathrm{t}}+\frac{1}{2} \nabla \psi^{1} \cdot \nabla \psi^{1}\right)-\rho\left(\boldsymbol{r}_{p}^{1} \cdot \nabla\left(\frac{\partial \psi^{1}}{\partial t}\right)\right)
$$

Table 1: Stokes' second-order wave velocity potential coefficients

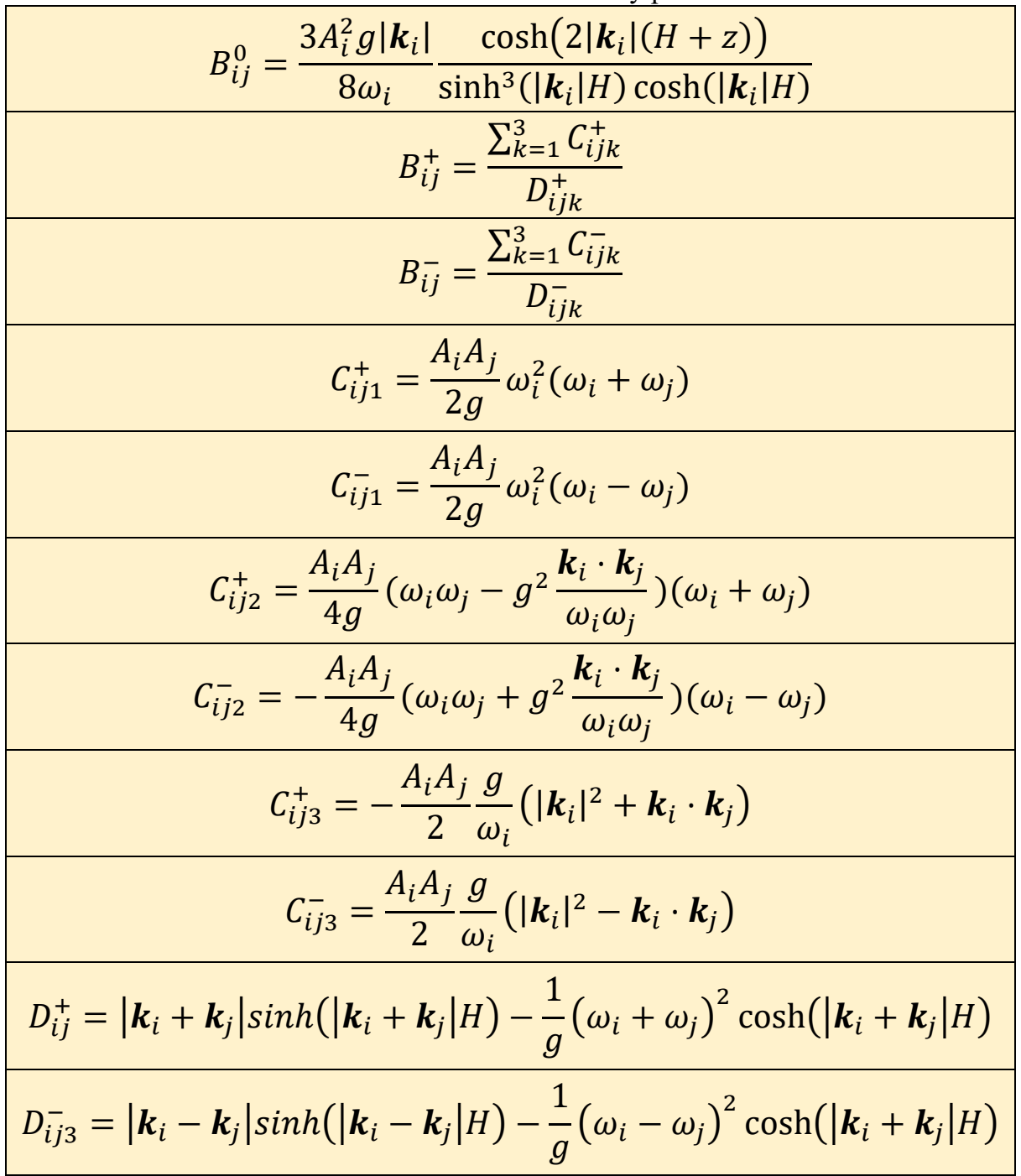

\section{Second-order diffraction-radiation wave problem}

The governing equations for the wave diffraction-radiation up to second-order are given by: $\Delta \phi^{1+2}=0$

in $\Omega$, 


$$
\begin{aligned}
& \frac{\partial \eta^{1+2}}{\partial t}-\frac{\partial \phi^{1+2}}{\partial z}=\eta^{1} \frac{\partial^{2} \phi^{1}}{\partial z^{2}}+\eta^{1} \frac{\partial^{2} \psi^{1}}{\partial z^{2}}+\zeta^{1} \frac{\partial^{2} \phi^{1}}{\partial z^{2}} \\
& -\frac{\partial \phi^{1}}{\partial x} \frac{\partial \eta^{1}}{\partial x}-\frac{\partial \phi^{1}}{\partial y} \frac{\partial \eta^{1}}{\partial y}-\frac{\partial \phi^{1}}{\partial x} \frac{\partial \zeta^{1}}{\partial x} \quad \text { in } z=0 \\
& -\frac{\partial \phi^{1}}{\partial y} \frac{\partial \zeta^{1}}{\partial y}-\frac{\partial \psi^{1}}{\partial x} \frac{\partial \eta^{1}}{\partial x}-\frac{\partial \psi^{1}}{\partial y} \frac{\partial \eta^{1}}{\partial y} \\
& \frac{\partial \phi^{1+2}}{\partial t}+\frac{P_{f s}}{\rho}+g \eta^{1+2}=-\eta^{1} \frac{\partial}{\partial z}\left(\frac{\partial \phi^{1}}{\partial t}\right)-\zeta^{1} \frac{\partial}{\partial z}\left(\frac{\partial \phi^{1}}{\partial t}\right) \\
& -\eta^{1} \frac{\partial}{\partial z}\left(\frac{\partial \psi^{1}}{\partial t}\right)-\frac{1}{2} \nabla \phi^{1} \cdot \nabla \phi^{1}-\nabla \psi^{1} \cdot \nabla \phi^{1} \\
& \boldsymbol{v}_{\phi}^{2} \cdot \boldsymbol{n}_{p}^{0}+\boldsymbol{v}_{\phi}^{1} \cdot \boldsymbol{n}_{p}^{1}=-\left(\boldsymbol{v}_{p}^{1}+\boldsymbol{v}_{\psi}^{1}\right) \cdot \boldsymbol{n}_{p}^{1} \\
& -\left(\boldsymbol{v}_{p}^{2}+\boldsymbol{v}_{\psi}^{2}+\left(\boldsymbol{r}_{p}^{1} \cdot \nabla\right)\left(\boldsymbol{v}_{\phi}^{1}+\boldsymbol{v}_{\psi}^{1}\right)\right) \cdot \boldsymbol{n}_{p}^{0} \\
& \boldsymbol{v}_{\phi}^{1+2} \cdot \boldsymbol{n}_{p}=-\boldsymbol{v}_{\psi}^{1+2} \cdot \boldsymbol{n}_{p} \\
& \text { in } z=0 \text {, } \\
& \text { on } P \in S_{B}^{0} \text {, } \\
& \text { on } P \in S_{S} \text {, }
\end{aligned}
$$

and the fluid pressure at a point $\mathrm{P}$ on the body surface is given by:

$$
P_{p}^{1+2}=P_{p h}^{0}+P_{p h}^{1+2}+P_{p \psi}^{1+2}+P_{p \phi}^{1+2}
$$

where $P_{p h}^{0}=-\rho g z, P_{p h}^{1+2}=-\rho g r_{p z}^{1+2}$ and:

$$
P_{p \phi}^{1+2}=-\rho \frac{\partial \phi^{1+2}}{\partial t}-\rho \frac{1}{2} \nabla \phi^{1} \cdot \nabla \phi^{1}-\rho \nabla \psi^{1} \cdot \nabla \phi^{1}-\rho r_{p}^{1} \cdot \nabla\left(\frac{\partial \phi^{1}}{\partial t}\right)
$$

\subsection{Numerical models for seakeeping problems}

\subsubsection{Glossary}

$\phi \quad$ Diffraction-radiation velocity potential

$\eta \quad$ Diffraction-radiation wave elevation

$P_{f s} \quad$ Free surface pressure

$\Gamma_{\mathrm{R}} \quad$ Surface limiting the computational domain in the horizontal directions

$\mathrm{R}^{1} \quad$ First-order source terms in combined FS BC for second-order solution

$\mathrm{S}^{1} \quad$ First-order source terms in kinematic BC for second-order solution

$\overline{\overline{\mathbf{L}}} \quad$ Laplacian FEM matrix

$\boldsymbol{\phi}$ Vector of diffraction-radiation velocity potential at nodes

$\mathbf{b}^{B} \quad$ FEM Neumann body boundary condition

$\mathbf{b}^{R} \quad$ FEM Neumann radiation boundary condition

$\mathbf{b}^{Z_{0}} \quad$ FEM Neumann free surface boundary condition

$\mathbf{b}^{Z_{H}} \quad$ FEM Neumann bottom boundary condition

$\Delta t \quad$ Time step

$\kappa(\boldsymbol{x})$ Wave absorption coefficient 


\subsubsection{Finite element formulation}

Section 2.2 presents the formulation based on the finite element method (FEM) to solve the system of equations governing the wave diffraction-radiation problem. This formulation has been developed to be used in conjunction with unstructured meshes in order to enhance geometry flexibility and speed up the initial modelling time.

Let $Q_{h}^{*}$ be the finite element space to interpolate functions, constructed in the usual manner. From this space, we can construct the subspace $Q_{h, \phi}^{*}$, that incorporates the Dirichlet conditions for the potential $\phi$. The space of test functions, denoted by $Q_{h}$, is constructed as $Q_{h, \phi}$, but with functions vanishing on the Dirichlet boundary. The weak form of the problem can be written as follows:

Find $\left[\phi_{h}\right] \in Q_{h, \phi}^{*}$, by solving the discrete variational problem:

$$
\begin{aligned}
\int_{\Omega} \nabla v_{h} \cdot \nabla \phi_{h} d \Omega= & \int_{\Gamma^{\mathrm{B}}} v_{h} \cdot \hat{\phi}_{n}^{B} d \Gamma+\int_{\Gamma^{\mathrm{R}}} v_{h} \cdot \hat{\phi}_{n}^{R} d \Gamma \\
& +\int_{\Gamma^{\mathrm{Z}_{0}}} v_{h} \cdot \hat{\phi}_{n}^{Z_{0}} d \Gamma+\int_{\Gamma^{\mathrm{S}}} v_{h} \cdot \hat{\phi}_{h}^{S} d \Gamma \quad \forall v_{h} \in Q_{h},
\end{aligned}
$$

where $\hat{\phi}_{n}^{B}, \hat{\phi}_{n}^{R}, \hat{\phi}_{n}^{Z_{0}}$ and $\hat{\phi}_{n}^{S}$ are the potential normal gradients corresponding to the Neumann boundary conditions on bodies, radiation boundary, free surface and seabed surface, respectively. At this point, it is useful to introduce the associated matrix form of Eq. (2-64):

$$
\overline{\overline{\mathbf{L}}} \boldsymbol{\phi}=\mathbf{b}^{B}+\mathbf{b}^{R}+\mathbf{b}^{Z_{0}}+\mathbf{b}^{S}
$$

where $\overline{\overline{\mathbf{L}}}$ is the standard Laplacian matrix, and $\mathbf{b}^{B}, \mathbf{b}^{R}, \mathbf{b}^{Z_{0}}$, and $\mathbf{b}^{S}$ are the vectors resulting of integrating the corresponding boundary condition terms.

\subsubsection{Free surface boundary conditions}

Combining the kinematic and dynamic free surface boundary conditions, the free surface condition reads:

$$
\frac{\partial^{2} \phi}{\partial t^{2}}+g \frac{\partial \phi}{\partial z}+\frac{\partial}{\partial t}\left(\frac{P_{f s}}{\rho}\right)+\left\{Q^{1}\right\}=0,
$$

and is implemented as a Neumann boundary condition that fulfils the flux boundary integral:

$$
\mathbf{b}^{Z_{0}}=\overline{\bar{M}}_{\Gamma^{z_{0}}}
$$

where $\overline{\bar{M}}_{\Gamma} \mathrm{z}_{0}$ is the corresponding boundary mass and $Q^{1}$ are the transfer terms from the first-order to the second-order problem (see Eqs. (2-58)-(2-59)):

$$
Q^{1}=\partial_{t} R^{1}-S^{1}
$$




$$
\begin{aligned}
R^{1}= & \eta^{1} \frac{\partial}{\partial z}\left(\frac{\partial \phi^{1}}{\partial t}\right)+\zeta^{1} \frac{\partial}{\partial z}\left(\frac{\partial \phi^{1}}{\partial t}\right)+\eta^{1} \frac{\partial}{\partial z}\left(\frac{\partial \psi^{1}}{\partial t}\right)+\frac{1}{2} \nabla \phi^{1} \cdot \nabla \phi^{1}+\nabla \psi^{1} \cdot \nabla \phi^{1} \\
S^{1}= & \frac{\partial \phi^{1}}{\partial \mathrm{x}} \frac{\partial \eta^{1}}{\partial x}+\frac{\partial \phi^{1}}{\partial \mathrm{y}} \frac{\partial \eta^{1}}{\partial y}+\frac{\partial \phi^{1}}{\partial \mathrm{x}} \frac{\partial \zeta^{1}}{\partial x}+\frac{\partial \phi^{1}}{\partial \mathrm{y}} \frac{\partial \zeta^{1}}{\partial y}+\frac{\partial \psi^{1}}{\partial \mathrm{x}} \frac{\partial \eta^{1}}{\partial x}+\frac{\partial \psi^{1}}{\partial \mathrm{y}} \frac{\partial \eta^{1}}{\partial y} \\
& -\eta^{1} \frac{\partial^{2} \phi^{1}}{\partial z^{2}}-\eta^{1} \frac{\partial^{2} \psi^{1}}{\partial z^{2}}-\zeta^{1} \frac{\partial^{2} \phi^{1}}{\partial z^{2}}
\end{aligned}
$$

where:

$$
\left(\partial_{t} R^{1}\right)^{n+1}=25 / 12\left(R^{1}\right)^{n+1}-4\left(R^{1}\right)^{n}+3\left(R^{1}\right)^{n-1}-4 / 3\left(R^{1}\right)^{n-2}+1 / 4\left(R^{1}\right)^{n-3} .
$$

Eq. (2-66) is discretized in time using the following numerical scheme:

$$
\begin{aligned}
\frac{\phi^{n+1}-2 \phi^{n}+\phi^{n-1}}{\Delta t^{2}}= & -\frac{1}{12} g\left(\phi_{z}^{n+1}+10 \phi_{z}^{n}+\phi_{z}^{n-1}\right)-\frac{P_{f s}^{n+1}-P_{f s}^{n-1}}{\rho 2 \Delta t} \\
& -\left\{\frac{1}{12}\left(\left(Q^{1}\right)^{n+1}+10\left(Q^{1}\right)^{n}+\left(Q^{1}\right)^{n-1}\right)\right\},
\end{aligned}
$$

where for the specific case where $P_{f s}=0$, the above scheme becomes a fourth order compact Padé scheme. The free surface elevation is discretized in time using the following fourth order in time numerical scheme:

$$
\eta^{n+1}=-\frac{1}{g \Delta t}\left(\frac{25}{12} \phi^{n+1}-4 \phi^{n}+3 \phi^{n-1}-\frac{4}{3} \phi^{n-2}+\frac{1}{4} \phi^{n-3}\right)-\frac{P_{f s}^{n+1}}{\rho g}\left\{-\left(R^{1}\right)^{n+1}\right\} .
$$

\subsubsection{Radiation condition and wave absorption}

Waves represented by $\phi$ are born at the bodies and propagate in all directions away from the bodies. These waves have to either be dissipated or to be let go out of the domain so they will not come back to interact with the bodies. Then a Sommerfeld radiation condition at the edge of the computational domain is introduced:

$$
\partial_{t} \phi+c \nabla \phi \cdot n_{R}=0 \quad \text { in } \Gamma_{R},
$$

where $\Gamma_{R}$ is the surface limiting the domain in the horizontal directions, and $c$ is a prescribed wave phase velocity. This radiation condition will let waves moving at velocity $c$ to escape out of the domain. The numerical scheme used to implement the radiation condition is

$$
\left(\phi_{n}^{R}\right)^{n+1}=-\frac{\phi^{n+1}-\phi^{n}}{c \Delta t} \text { in } \Gamma_{R}
$$

The prescribed phase velocity $c$ will be set for radiating those waves with the smallest frequency (largest wavelengths) considered in each specific case under study. However, waves with higher frequencies (smaller phase velocities) will not leave the domain at $\Gamma_{\mathrm{R}}$ and will be reflected. Hence, wave absorption is introduced into the dynamic free surface boundary condition by varying the pressure such that: 


$$
P_{f S}(\boldsymbol{x}, t)=\kappa(\boldsymbol{x}) \rho \frac{\partial \phi}{\partial z}
$$

Eq. (2-76) increases pressure when the free surface is moving upwards, while decreases the pressure when the free surface is moving downwards. Then energy is transferred from the waves to the atmosphere and waves are damped. However, the coefficient $\kappa(\boldsymbol{x})$ will be set to zero in the analysis area (near the bodies), so that damping will have no effect on the solution of the wave-body interaction problem.

\subsection{Hydrodynamic loads in seakeeping problems}

\subsubsection{Glossary}

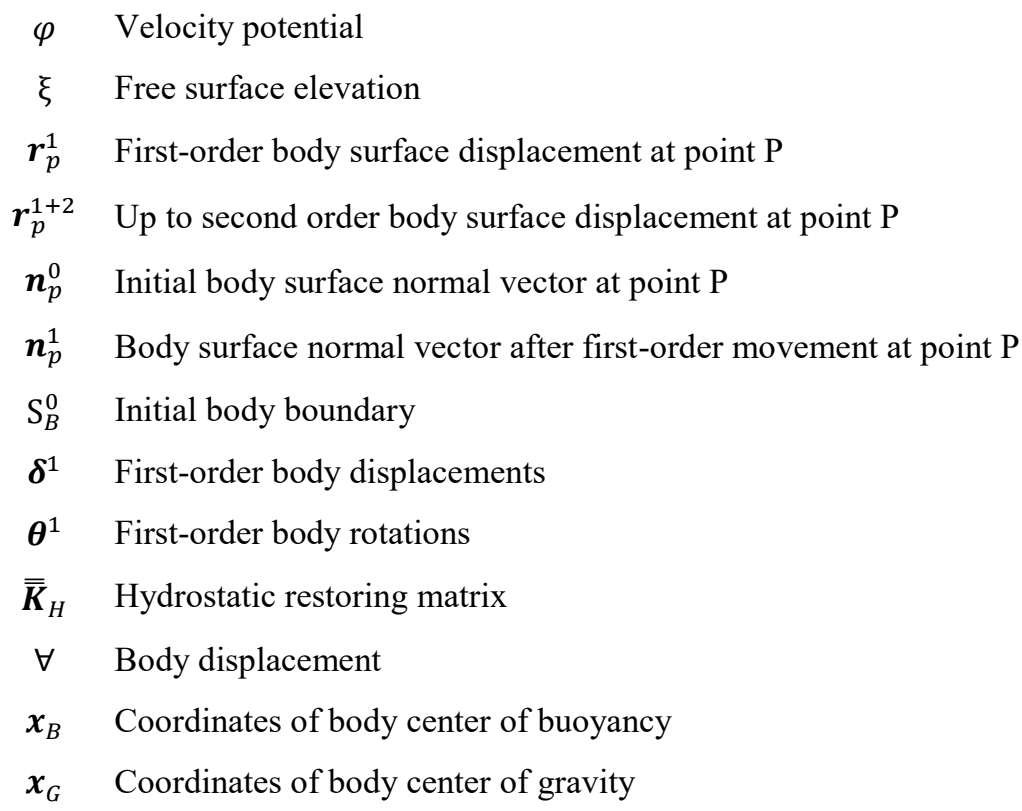

\subsubsection{Introduction}

The aim of section 2.3 is to obtain the hydrodynamic forces and moments from direct pressure integration. These loads are split in hydrostatic and dynamic components, obtained by integrating the hydrostatic and dynamic pressure respectively. Furthermore, loads are split into first-order and secondorder depending on the order of approximation used.

\subsubsection{First-order loads}

Hydrodynamic forces and moments are obtained from direct pressure integration over the body surface. The body gravity center will be used as a reference for body movements and moments acting on it. The first-order forces and moments are: 


$$
\begin{aligned}
& \boldsymbol{F}^{1}=\int_{S_{B}^{0}} P_{p}^{1} \boldsymbol{n}_{p}^{0} d s=\boldsymbol{F}_{H}^{0}+\boldsymbol{F}_{H}^{1}+\boldsymbol{F}_{D}^{1}, \\
& \boldsymbol{M}^{1}=\int_{S_{B}^{0}} P_{p}^{1}\left(\boldsymbol{x}_{G}-\boldsymbol{x}_{p}\right) \times \boldsymbol{n}_{p}^{0} d s=\boldsymbol{M}_{H}^{0}+\boldsymbol{M}_{H}^{1}+\boldsymbol{M}_{D}^{1},
\end{aligned}
$$

where sub-index H stands for hydrostatic loads and D stands for dynamic loads. The hydrostatic loads are split as follows:

$$
\begin{aligned}
& \boldsymbol{F}_{H}^{0}=-\int_{\mathrm{S}_{B}^{0}} \rho g z \boldsymbol{n}_{p}^{0} d s=\rho g \forall, \\
& \boldsymbol{M}_{H}^{0}=-\int_{\mathrm{S}_{B}^{0}} \rho g z \overrightarrow{\boldsymbol{G}^{0} \boldsymbol{P}^{0}} \times \boldsymbol{n}_{p}^{0} d s, \\
& \boldsymbol{F}_{H}^{1}=-\int_{\mathrm{S}_{B}^{0}} \rho g r_{p z}^{1} \boldsymbol{n}_{p}^{0} d s, \\
& =-\int_{\mathrm{S}_{B}^{0}} \rho g r_{p z}^{1} \overrightarrow{\boldsymbol{G}^{0} \boldsymbol{P}^{0}} \times \boldsymbol{n}_{p}^{0} d s,
\end{aligned}
$$

where $\forall$ is the body displacement. Hydrostatic forces and moments are obtained via the hydrostatic restoring matrix $\overline{\overline{\mathbf{K}}}_{H}$ :

$$
\left[\begin{array}{c}
\boldsymbol{F}_{H}^{1} \\
\boldsymbol{M}_{H}^{1}
\end{array}\right]=\overline{\overline{\boldsymbol{K}}}_{H}\left[\begin{array}{l}
\boldsymbol{\delta}^{1} \\
\boldsymbol{\theta}^{1}
\end{array}\right]=\left[\begin{array}{c}
\overline{\overline{\boldsymbol{K}}}_{H}^{F} \\
\overline{\overline{\boldsymbol{K}}}_{H}^{M}
\end{array}\right]\left[\begin{array}{l}
\boldsymbol{\delta}^{1} \\
\boldsymbol{\theta}^{1}
\end{array}\right] .
$$

The body displacement, center of buoyancy, and restoring matrix are obtained as follows:

$$
\begin{aligned}
& \forall=-\int_{S_{B}^{0}} z n_{p z} d s, \\
& x_{B}=-\frac{1}{2 \forall} \int_{S_{B}^{0}} x^{2} n_{p x} d s ; y_{B}=-\frac{1}{2 \forall} \int_{S_{B}^{0}} y^{2} n_{p y} d s ; z_{B}=-\frac{1}{2 \forall} \int_{S_{B}^{0}} z^{2} n_{p z} d s, \\
& K_{H}(3,3)=\rho g \int_{S_{B}^{0}} n_{p z} d s, \\
& K_{H}(3,4)=K_{H}(4,3)=\rho g \int_{S_{B}^{0}}\left(y_{p}-y_{G}\right) n_{p z} d s, \\
& K_{H}(3,5)=K_{H}(5,3)=-\rho g \int_{S_{B}^{0}}\left(x_{p}-x_{G}\right) n_{p z} d s, \\
& K_{H}(4,4)=\rho g \int_{S_{B}^{0}}\left(y_{p}-y_{G}\right)^{2} n_{p z} d s+\rho g \forall\left(z_{B}-z_{G}\right), \\
& K_{H}(4,5)=K_{H}(5,4)=-\rho g \int_{S_{B}^{0}}\left(x_{p}-x_{G}\right)\left(y_{p}-y_{G}\right) n_{p z} d s, \\
& K_{H}(4,6)=-\rho g \forall\left(x_{B}-x_{G}\right),
\end{aligned}
$$




$$
\begin{aligned}
& K_{H}(5,5)=\rho g \int_{S_{B}^{0}}\left(x_{p}-x_{G}\right)^{2} n_{p Z} d s+\rho g \forall\left(z_{B}-z_{G}\right), \\
& K_{H}(5,6)=-\rho g \forall\left(y_{B}-y_{G}\right),
\end{aligned}
$$

where B stands for the body center of buoyancy, and $\mathrm{G}$ for the body center of gravity. For all other values of the indices $(\mathrm{i}, \mathrm{j}), K_{H}(i, j)=0$. The dynamic loads are computed as:

$$
\begin{aligned}
& \boldsymbol{F}_{D}^{1}=-\int_{S_{B}^{0}} \rho \frac{\partial \varphi^{1}}{\partial t} \boldsymbol{n}_{p}^{0} d s \\
& \boldsymbol{M}_{D}^{1}=-\int_{S_{B}^{0}} \rho \frac{\partial \varphi^{1}}{\partial t} \overrightarrow{\boldsymbol{G}^{0} \boldsymbol{P}^{0}} \times \boldsymbol{n}_{p}^{0} d s .
\end{aligned}
$$

\subsubsection{Second-order loads}

Up to second-order forces and moments are split as:

$$
\begin{aligned}
& \boldsymbol{F}^{1+2}=\boldsymbol{F}_{H}^{0}+\boldsymbol{F}_{H}^{1}+\boldsymbol{F}_{H}^{2}+\boldsymbol{F}_{D}^{1}+\boldsymbol{F}_{D}^{2}, \\
& \boldsymbol{M}^{1+2}=\boldsymbol{M}_{H}^{0}+\boldsymbol{M}_{H}^{1}+\boldsymbol{M}_{H}^{2}+\boldsymbol{M}_{D}^{1}+\boldsymbol{M}_{D}^{2},
\end{aligned}
$$

where the hydrostatic loads are:

$$
\begin{aligned}
& \boldsymbol{F}_{H}^{1+2}=-\int_{S_{B}^{0}} \rho g\left(z_{p}+r_{p z}^{1+2}\right) \boldsymbol{n}_{p}^{1} d s \\
& =\boldsymbol{F}_{H}^{0}+\boldsymbol{F}_{H}^{1}+\boldsymbol{\theta}^{1} \times \boldsymbol{F}_{H}^{1}+\overline{\overline{\boldsymbol{K}}}_{H}^{F}\left[\begin{array}{l}
\boldsymbol{\delta}^{2} \\
\boldsymbol{\theta}^{2}
\end{array}\right]-\int_{S_{B}^{0}} \rho g\left(\overline{\overline{\boldsymbol{H}}} \overrightarrow{\boldsymbol{R}^{0} \boldsymbol{P}^{0}}\right)_{z} \boldsymbol{n}_{p}^{0} d s, \\
& \boldsymbol{M}_{H}^{1+2}=-\int_{S_{B}^{0}} \rho g\left(z_{p}+r_{p z}^{1+2}\right) \overrightarrow{\boldsymbol{G}^{0} \boldsymbol{P}^{0}} \times \boldsymbol{n}_{p}^{1} d s \\
& \quad=\boldsymbol{M}_{H}^{0}+\boldsymbol{M}_{H}^{1}+\boldsymbol{\theta}^{1} \times \boldsymbol{M}_{H}^{1}+\overline{\overline{\boldsymbol{K}}}_{H}^{M}\left[\begin{array}{l}
\boldsymbol{\delta}^{2} \\
\boldsymbol{\theta}^{2}
\end{array}\right]-\int_{S_{B}^{0}} \rho g\left(\overline{\overline{\boldsymbol{H}}} \overrightarrow{\boldsymbol{R}^{0} \boldsymbol{P}^{0}}\right)_{z} \overrightarrow{\boldsymbol{G}^{0} \boldsymbol{P}^{0}} \times \boldsymbol{n}_{p}^{0} d s,
\end{aligned}
$$

then:

$$
\begin{aligned}
& \boldsymbol{F}_{H}^{2}=\overline{\overline{\boldsymbol{K}}}_{H}^{F}\left[\begin{array}{l}
\boldsymbol{\delta}^{2} \\
\boldsymbol{\theta}^{2}
\end{array}\right]+\boldsymbol{\theta}^{1} \times \boldsymbol{F}_{H}^{1}-\int_{S_{B}^{0}} \rho g\left(\overline{\overline{\boldsymbol{H}}} \overrightarrow{\boldsymbol{R}^{0} \boldsymbol{P}^{0}}\right)_{z} \boldsymbol{n}_{p}^{0} d s, \\
& \boldsymbol{M}_{H}^{2}=\overline{\overline{\boldsymbol{K}}}_{H}^{M}\left[\begin{array}{l}
\boldsymbol{\delta}^{2} \\
\boldsymbol{\theta}^{2}
\end{array}\right]+\boldsymbol{\theta}^{1} \times \boldsymbol{M}_{H}^{1}-\int_{S_{B}^{0}} \rho g\left(\overline{\overline{\boldsymbol{H}}} \overrightarrow{\boldsymbol{R}^{0} \boldsymbol{P}^{0}}\right)_{z} \overrightarrow{\boldsymbol{G}^{0} \boldsymbol{P}^{0}} \times \boldsymbol{n}_{p}^{0} d s .
\end{aligned}
$$

The dynamic loads up to second order are split in four components:

$$
\begin{aligned}
& \boldsymbol{F}_{D}^{1+2}=\boldsymbol{F}_{D}^{1}+\boldsymbol{F}_{D}^{2}=\boldsymbol{F}_{D}^{1}+\boldsymbol{F}_{D 1}^{2}+\boldsymbol{F}_{D 2}^{2}+\boldsymbol{F}_{D 3}^{2}+\boldsymbol{F}_{D 4}^{2}, \\
& \boldsymbol{M}_{D}^{1+2}=\boldsymbol{M}_{D}^{1}+\boldsymbol{M}_{D}^{2}=\boldsymbol{M}_{D}^{1}+\boldsymbol{M}_{D 1}^{2}+\boldsymbol{M}_{D 2}^{2}+\boldsymbol{M}_{D 3}^{2}+\boldsymbol{M}_{D 4}^{2},
\end{aligned}
$$


where

$$
\begin{aligned}
& \boldsymbol{F}_{D 1}^{2}=-\rho \int_{S_{B}^{0}} \frac{\partial \varphi^{2}}{\partial t} \boldsymbol{n}_{p}^{0} d s+\boldsymbol{\theta}^{1} \times \boldsymbol{F}_{D}^{1}, \\
& \boldsymbol{F}_{D 2}^{2}=-\rho \int_{S_{B}^{0}}\left(\boldsymbol{r}_{p}^{1} \cdot \nabla\left(\frac{\partial \varphi^{1}}{\partial t}\right)\right) \boldsymbol{n}_{p}^{0} d s, \\
& \boldsymbol{F}_{D 3}^{2}=-\frac{1}{2} \rho \int_{S_{B}^{0}}\left(\nabla \varphi^{1} \cdot \nabla \varphi^{1}\right) \boldsymbol{n}_{p}^{0} d s, \\
& \boldsymbol{F}_{D 4}^{2}=-\frac{1}{2} \rho g \int_{\Gamma_{B}^{0}}\left(\xi^{1}-r_{p z}^{1}\right)^{2} \frac{\boldsymbol{n}_{p}^{0}}{\sqrt{1-n_{p z}^{0}}} d l, \\
& \boldsymbol{M}_{D 1}^{2}=-\rho \int_{S_{b}^{0}} \frac{\partial \varphi^{2}}{\partial t} \overrightarrow{\boldsymbol{G}^{0} \boldsymbol{P}^{0}} \times \boldsymbol{n}_{p}^{0} d s+\boldsymbol{\theta}^{1} \times \boldsymbol{M}_{D}^{1}, \\
& \boldsymbol{M}_{D 2}^{2}=-\rho \int_{S_{b}^{0}}\left(\boldsymbol{r}_{p}^{1} \cdot \nabla\left(\frac{\partial \varphi^{1}}{\partial t}\right)\right) \overrightarrow{\boldsymbol{G}^{0} \boldsymbol{P}^{0}} \times \boldsymbol{n}_{p}^{0} d s, \\
& \boldsymbol{M}_{D 3}^{2}=-\frac{1}{2} \rho \int_{\mathrm{S}_{b}^{0}}\left(\nabla \varphi^{1} \cdot \nabla \varphi^{1}\right) \overrightarrow{\boldsymbol{G}^{0} \boldsymbol{P}^{0}} \times \boldsymbol{n}_{p}^{0} d s, \\
& \boldsymbol{M}_{D 4}^{2}=-\frac{1}{2} \rho g \int_{\Gamma_{B}^{0}}\left(\xi^{1}-r_{p z}^{1}\right)^{2} \overrightarrow{\boldsymbol{G}^{0} \boldsymbol{P}^{0}} \times \frac{\boldsymbol{n}_{p}^{0}}{\sqrt{1-n_{p z}^{0}{ }^{2}}} d l .
\end{aligned}
$$

\subsubsection{Mean drift loads}

Being the first-order and second-order responses harmonic, and taking time average, the following relations holds:

$$
\begin{aligned}
& <\boldsymbol{F}^{1}>=0 \\
& <\boldsymbol{M}^{1}>=0 \\
& <\boldsymbol{F}_{H}^{2}>=<\boldsymbol{\theta}^{1} \times \boldsymbol{F}_{H}^{1}>-<\int_{S_{B}^{0}} \rho g\left(\overline{\overline{\boldsymbol{H}}} \overrightarrow{\boldsymbol{R}^{0} \boldsymbol{P}^{0}}\right)_{z} \boldsymbol{n}_{p}^{0} d s> \\
& <\boldsymbol{M}_{H}^{2}>=<\boldsymbol{\theta}^{1} \times \boldsymbol{M}_{H}^{1}>-<\int_{S_{B}^{0}} \rho g\left(\overline{\overline{\boldsymbol{H}}} \overrightarrow{\boldsymbol{R}^{0} \boldsymbol{P}^{0}}\right)_{z} \overrightarrow{\boldsymbol{G}^{0} \boldsymbol{P}^{0}} \times \boldsymbol{n}_{p}^{0} d s>, \\
& <\boldsymbol{F}_{D 1}^{2}>=<\boldsymbol{\theta}^{1} \times \boldsymbol{F}_{D}^{1}> \\
& <\boldsymbol{F}_{D 2}^{2}>=-\rho<\int_{S_{B}^{0}}\left(\boldsymbol{r}_{p}^{1} \cdot \nabla\left(\frac{\partial \varphi^{1}}{\partial t}\right)\right) \boldsymbol{n}_{p}^{0} d s> \\
& <\boldsymbol{F}_{D 3}^{2}>=-\frac{1}{2} \rho<\int_{S_{B}^{0}}\left(\nabla \varphi^{1} \cdot \nabla \varphi^{1}\right) \boldsymbol{n}_{p}^{0} d s>
\end{aligned}
$$




$$
\begin{aligned}
& <\boldsymbol{F}_{D 4}^{2}>=-\frac{1}{2} \rho g<\int_{\Gamma_{B}^{0}}\left(\xi^{1}-r_{p z}^{1}\right)^{2} \frac{\boldsymbol{n}_{p}^{0}}{\sqrt{1-n_{p z}^{0}}} d l>, \\
& <\boldsymbol{M}_{D 1}^{2}>=<\boldsymbol{\theta}^{1} \times \boldsymbol{M}_{D}^{1}> \\
& <\boldsymbol{M}_{D 2}^{2}>=-\rho<\int_{S_{b}^{0}}\left(\boldsymbol{r}_{p}^{1} \cdot \nabla\left(\frac{\partial \varphi^{1}}{\partial t}\right)\right) \overrightarrow{\boldsymbol{G}^{0} \boldsymbol{P}^{0}} \times \boldsymbol{n}_{p}^{0} d s> \\
& <\boldsymbol{M}_{D 3}^{2}>=-\frac{1}{2} \rho<\int_{S_{b}^{0}}\left(\nabla \varphi^{1} \cdot \nabla \varphi^{1}\right) \overrightarrow{\boldsymbol{G}^{0} \boldsymbol{P}^{0}} \times \boldsymbol{n}_{p}^{0} d s>, \\
& <\boldsymbol{M}_{D 4}^{2}>=-\frac{1}{2} \rho g<\int_{\Gamma_{B}^{0}}\left(\xi^{1}-r_{p Z}^{1}\right)^{2} \frac{\boldsymbol{G}^{0} \boldsymbol{P}^{0}}{\boldsymbol{n}_{p}^{0}} \times \frac{\sqrt{1-n_{p z}^{0}}}{\sqrt{1-}} d l>.
\end{aligned}
$$

Second-order terms with non-zero time average depend on first-order quantities. Hence secondorder drifting loads only depend on the first-order problem solution. 


\section{Chapter 3. WAVE RESISTANCE PROBLEMS}

\subsection{Mathematical models for wave resistance problems}

\subsubsection{Glossary}

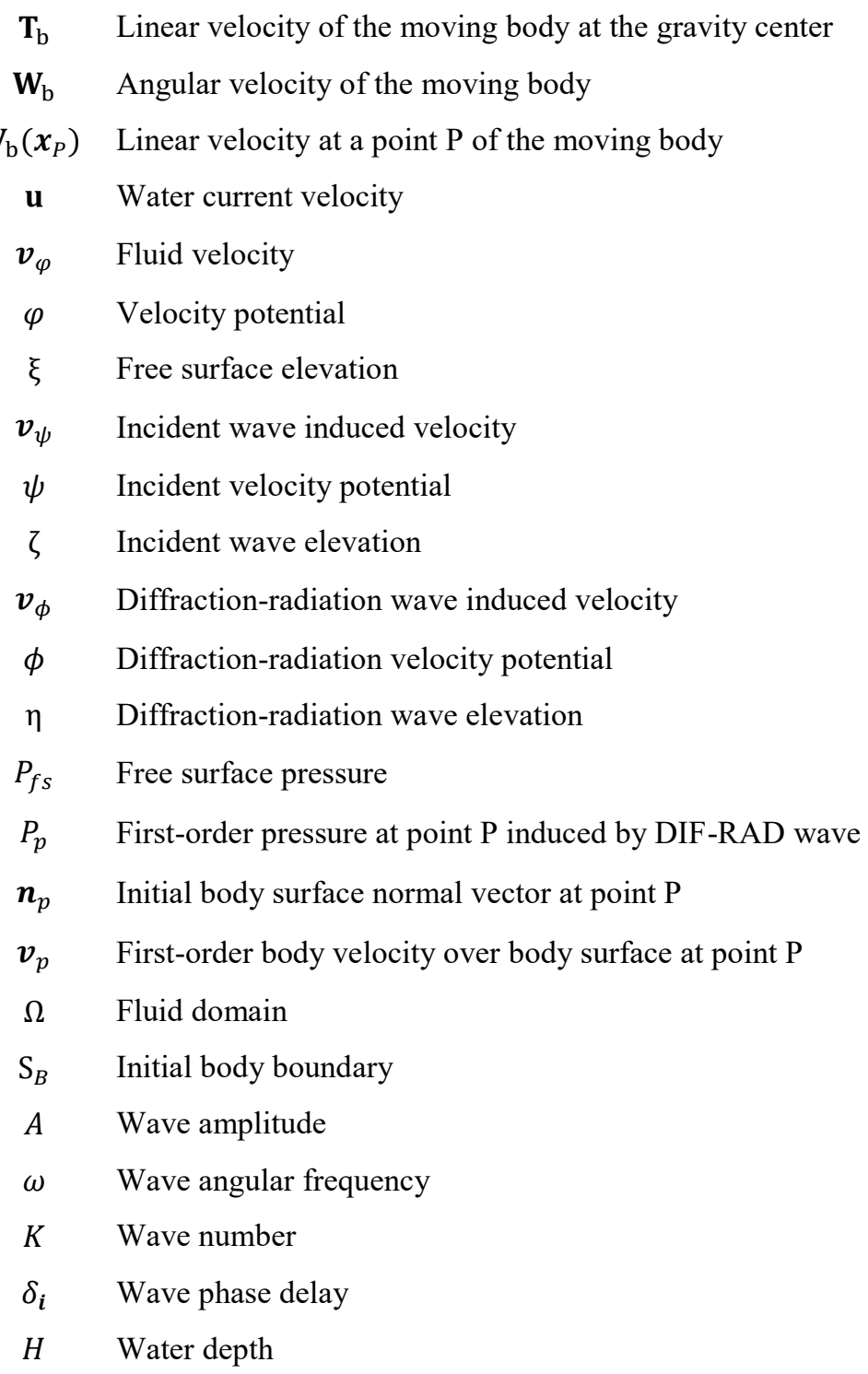

\subsubsection{Introduction}

In this section the problem discussed is that of a body moving across the fluid domain in the presence of waves and water currents. Both, body velocity and water current will be assumed to be much larger than velocities induced by waves. 


\subsubsection{Problem statement}

We consider the first-order diffraction-radiation problem of a body moving on the horizontal plane. As in Chapter 2, incompressible and irrotational flow are assumed, being $\varphi$ the velocity potential. The following assumptions on the order of magnitude of the velocity components and free surface elevation $\xi$ are made:

$$
\begin{array}{llll}
\left|\mathbf{V}_{\mathrm{b}} \sim O(1)\right| & |\mathbf{u} \sim O(1)| & \varphi_{x} \sim O(1) & \varphi_{y} \sim O(1) \\
\varphi_{z} \sim O(\epsilon) & \varphi_{\alpha \beta} \sim O(\epsilon) & \xi_{x} \sim O(\epsilon) & \xi_{y} \sim O(\epsilon)
\end{array}
$$

Based on the previous assumptions, the governing equations for the first-order diffraction-radiation wave problem are:

$$
\begin{array}{lll}
\Delta \varphi=0 & \text { in } \Omega & \begin{array}{l}
\text { incompressible and } \\
\text { irrotational flow, }
\end{array} \\
\frac{\partial \varphi}{\partial \mathrm{t}}+\boldsymbol{u} \cdot \nabla_{\mathrm{h}} \varphi+\frac{1}{2} \nabla_{\mathrm{h}} \varphi \cdot \nabla_{\mathrm{h}} \varphi+\frac{P_{f s}}{\rho}+g \xi=0 & \text { on } z=0 & \begin{array}{l}
\text { dynamic boundary } \\
\text { condition, }
\end{array} \\
\frac{\partial \xi}{\partial t}+\left(\boldsymbol{u}+\nabla_{\mathrm{h}} \varphi\right) \cdot \nabla_{\mathrm{h}} \xi-\frac{\partial \varphi}{\partial \mathrm{z}}=0 & \text { on } z=0 & \begin{array}{l}
\text { kinematic boundary } \\
\text { condition, }
\end{array} \\
\boldsymbol{v}_{p} \cdot \boldsymbol{n}_{p}+\left(\boldsymbol{u}+\boldsymbol{v}_{\varphi}\right) \cdot \boldsymbol{n}_{p}=0 & \text { on } \mathrm{S}_{B}^{0} & \text { body boundary condition, } \\
\frac{\partial \varphi}{\partial \mathrm{z}}=0 & \text { on } z=-H & \text { wall boundary condition, }
\end{array}
$$

and the pressure at an arbitrary point $\mathrm{P}$ of the fluid domain is:

$$
P_{p}=-\rho\left(\frac{\partial \varphi}{\partial t}+\boldsymbol{u} \cdot \nabla_{\mathrm{h}} \varphi+\frac{1}{2} \nabla \varphi \cdot \nabla \varphi+g z_{p}\right) .
$$

\subsubsection{Velocity potential decomposition}

In order to solve the governing equations (Eqs.(3-2)-(3-6)), a velocity potential decomposition is introduced. The total velocity potential can be decomposed as:

$$
\begin{aligned}
& \varphi=\psi+\phi, \\
& \xi=\zeta+\eta,
\end{aligned}
$$

where $\psi$ and $\zeta$ are the incident wave potential and free surface elevation respectively, and $\phi$ and $\eta$ are the diffraction-radiation velocity potential and free surface elevation respectively. Then, we can split the governing equations into the following sets of equations: 


\subsubsection{Set 1: incident waves}

The first set of equations describes the incident waves:

$$
\begin{array}{ll}
\Delta \psi=0 & \text { in } \Omega, \\
\frac{\partial \psi}{\partial \mathrm{t}}+\boldsymbol{u} \cdot \nabla_{\mathrm{h}} \psi+g \zeta=0 & \text { on } z=0, \\
\frac{\partial \xi}{\partial t}+\boldsymbol{u} \cdot \nabla_{\mathrm{h}} \zeta-\frac{\partial \varphi}{\partial \mathrm{z}}=0 & \text { on } z=0, \\
\frac{\partial \psi}{\partial \mathrm{z}}=0 & \text { on } z=-H .
\end{array}
$$

This set of equations has the following analytical solution (Airy waves):

$$
\begin{aligned}
& \psi=\sum_{i} \frac{A_{i} g}{\omega_{i}} \frac{\cosh \left(\left|\boldsymbol{k}_{i}\right|(H+z)\right)}{\cosh \left(\left|\boldsymbol{k}_{i}\right| H\right)} \sin \left(\boldsymbol{k}_{i}(\boldsymbol{x}-\boldsymbol{u} t)-\omega_{i} t+\delta_{i}\right), \\
& \zeta=\sum_{i} A_{i} \cos \left(\boldsymbol{k}_{i}(\boldsymbol{x}-\boldsymbol{u} t)-\omega_{i} t+\delta_{i}\right) .
\end{aligned}
$$

\subsubsection{Set 2: governing equations of wave diffraction-radiation problem}

The second set of equations describes the diffraction-radiation wave problem:

$$
\begin{array}{ll}
\Delta \phi=0 & \text { in } \Omega, \\
\frac{\partial \phi}{\partial \mathrm{t}}+\boldsymbol{u} \cdot \nabla_{\mathrm{h}} \phi+\frac{1}{2} \nabla_{\mathrm{h}} \phi \cdot \nabla_{\mathrm{h}} \phi+\nabla_{\mathrm{h}} \psi \cdot \nabla_{\mathrm{h}} \phi+\frac{1}{2} \nabla_{\mathrm{h}} \psi \cdot \nabla_{\mathrm{h}} \psi+\frac{P_{f s}}{\rho}+g \eta=0 & \text { on } z=0, \\
\frac{\partial \eta}{\partial t}+\left(\boldsymbol{u}+\nabla_{\mathrm{h}} \phi+\nabla_{\mathrm{h}} \psi\right) \cdot \nabla_{\mathrm{h}} \eta+\left(\nabla_{\mathrm{h}} \phi+\nabla_{\mathrm{h}} \psi\right) \cdot \nabla_{\mathrm{h}} \zeta-\frac{\partial \phi}{\partial \mathrm{z}}=0 & \text { on } z=0, \\
\boldsymbol{v}_{p} \cdot \boldsymbol{n}_{p}+\left(\boldsymbol{u}+\boldsymbol{v}_{\psi}+\boldsymbol{v}_{\phi}\right) \cdot \boldsymbol{n}_{p}=0 & \text { on } \mathrm{S}_{B}^{0}, \\
\frac{\partial \phi}{\partial \mathrm{z}}=0 & \text { in } z=-H .
\end{array}
$$

From first-order wave theory, we know that $\psi_{\alpha} \sim O(\epsilon)$ and $\zeta_{\alpha} \sim O(\epsilon)$. Then, the terms $\frac{1}{2} \nabla_{\mathrm{h}} \psi$. $\nabla_{\mathrm{h}} \psi \sim O\left(\epsilon^{2}\right), \nabla_{\mathrm{h}} \psi \cdot \nabla_{\mathrm{h}} \zeta \sim O\left(\epsilon^{2}\right)$, and $\nabla_{\mathrm{h}} \psi \cdot \nabla_{\mathrm{h}} \eta \sim O\left(\epsilon^{2}\right)$. Neglecting these terms in the second set of equations (Eqs. (3-16)-(3-20)), the governing equations for the first-order diffraction-radiation wave problem become:

$$
\begin{array}{ll}
\Delta \phi=0 & \text { in } \Omega, \\
\frac{\partial \phi}{\partial \mathrm{t}}+\boldsymbol{u} \cdot \nabla_{\mathrm{h}} \phi+\frac{1}{2} \nabla_{\mathrm{h}} \phi \cdot \nabla_{\mathrm{h}} \phi+\nabla_{\mathrm{h}} \psi \cdot \nabla_{\mathrm{h}} \phi+\frac{P_{f s}}{\rho}+g \eta=0 & \text { on } z=0, \\
\frac{\partial \eta}{\partial t}+\left(\boldsymbol{u}+\nabla_{\mathrm{h}} \phi\right) \cdot \nabla_{\mathrm{h}} \eta+\nabla_{\mathrm{h}} \phi \cdot \nabla_{\mathrm{h}} \zeta-\frac{\partial \phi}{\partial \mathrm{z}}=0 & \text { on } z=0, \\
\nabla_{\mathrm{h}} \phi \cdot \boldsymbol{n}_{p}=-\left(\boldsymbol{v}_{p}+\boldsymbol{u}+\boldsymbol{v}_{\psi}\right) \cdot \boldsymbol{n}_{p} & \text { on } \mathrm{S}_{B}^{0}, \\
\frac{\partial \phi}{\partial \mathrm{z}}=0 & \text { on } z=-H,
\end{array}
$$


and the pressure induced at point $\mathrm{P}$ by diffracted and radiated waves is:

$$
P_{p}=-\rho\left(\frac{\partial \phi}{\partial t}+\left(\boldsymbol{u}+\nabla_{\mathrm{h}} \phi+\nabla_{\mathrm{h}} \psi\right) \cdot \nabla_{\mathrm{h}} \phi-\frac{1}{2} \nabla \phi \cdot \nabla \phi\right)
$$

Notice that the terms $\nabla_{\mathrm{h}} \psi \cdot \nabla_{\mathrm{h}} \phi$ and $\nabla_{\mathrm{h}} \phi \cdot \nabla_{\mathrm{h}} \zeta$ account for the deviation of the incident waves due to the fact that they are transported by a non-uniform flow field. Also, the terms $\nabla_{\mathrm{h}} \phi \cdot \nabla_{\mathrm{h}} \phi$ and $\nabla_{\mathrm{h}} \phi \cdot \nabla_{\mathrm{h}} \eta$ are not subject to any kind of linearization so far, which enables to simulate non-steady base flows.

\subsubsection{Governing equations in a moving frame of reference}

It is convenient to solve Eqs. (3-21)-(3-25) in a frame of reference fixed to the moving body rather than on the global frame of reference. Therefore, the aforementioned equations will be solved in a local frame of reference. Let be the two dimensional movement of the body: $\mathbf{V}_{\mathrm{b}}(\mathbf{x})=\mathbf{T}_{\mathrm{b}}+\mathbf{W}_{\mathrm{b}} \times\left(\mathbf{x}-\mathbf{x}_{\mathrm{G}}\right)$ , where $\mathbf{T}_{\mathrm{b}}$ and $\mathbf{W}_{\mathrm{b}}$ are the linear and angular velocity of the moving body. Figure 2 shows the global and local frame of reference. This frame of reference is assumed to match the global frame at time zero. For an observer sitting in the ship, he will observe the flow field around the ship given by the relative motion $\boldsymbol{U}_{b}(\boldsymbol{x})=\boldsymbol{u}-\boldsymbol{V}_{b}(\boldsymbol{x})$. Therefore, the governing equations in the local frame of reference become:

$$
\begin{array}{ll}
\Delta \phi=0 & \text { in } \Omega, \\
\frac{\partial \phi}{\partial \mathrm{t}}+\boldsymbol{U}_{b} \cdot \nabla_{\mathrm{h}} \phi+\frac{1}{2} \nabla_{\mathrm{h}} \phi \cdot \nabla_{\mathrm{h}} \phi+\nabla_{\mathrm{h}} \psi \cdot \nabla_{\mathrm{h}} \phi+\frac{P_{f s}}{\rho}+g \eta=0 & \text { on } z=0, \\
\frac{\partial \eta}{\partial t}+\left(\boldsymbol{U}_{b}+\nabla_{\mathrm{h}} \phi\right) \cdot \nabla_{\mathrm{h}} \eta+\nabla_{\mathrm{h}} \phi \cdot \nabla_{\mathrm{h}} \zeta-\frac{\partial \phi}{\partial \mathrm{z}}=0 & \text { on } z=0, \\
\nabla \phi \cdot \boldsymbol{n}_{p}=-\left(\boldsymbol{v}_{p}+\boldsymbol{u}+\boldsymbol{v}_{\psi}\right) \cdot \boldsymbol{n}_{p} & \text { on } \mathrm{S}_{B}^{0}, \\
\frac{\partial \phi}{\partial \mathrm{z}}=0 & \text { on } z=-H,
\end{array}
$$

and the pressure induced by diffracted and radiated waves is:

$$
P_{p}=-\rho\left(\frac{\partial \phi}{\partial t}+\left(\boldsymbol{U}_{b}+\nabla_{\mathrm{h}} \phi+\nabla_{\mathrm{h}} \psi\right) \cdot \nabla_{\mathrm{h}} \phi-\frac{1}{2} \nabla_{\mathrm{h}} \phi \cdot \nabla_{\mathrm{h}} \phi\right) \quad \text { in } \Omega,
$$

where the incident wave potential and incident wave elevation must be transformed to the local frame of reference. 


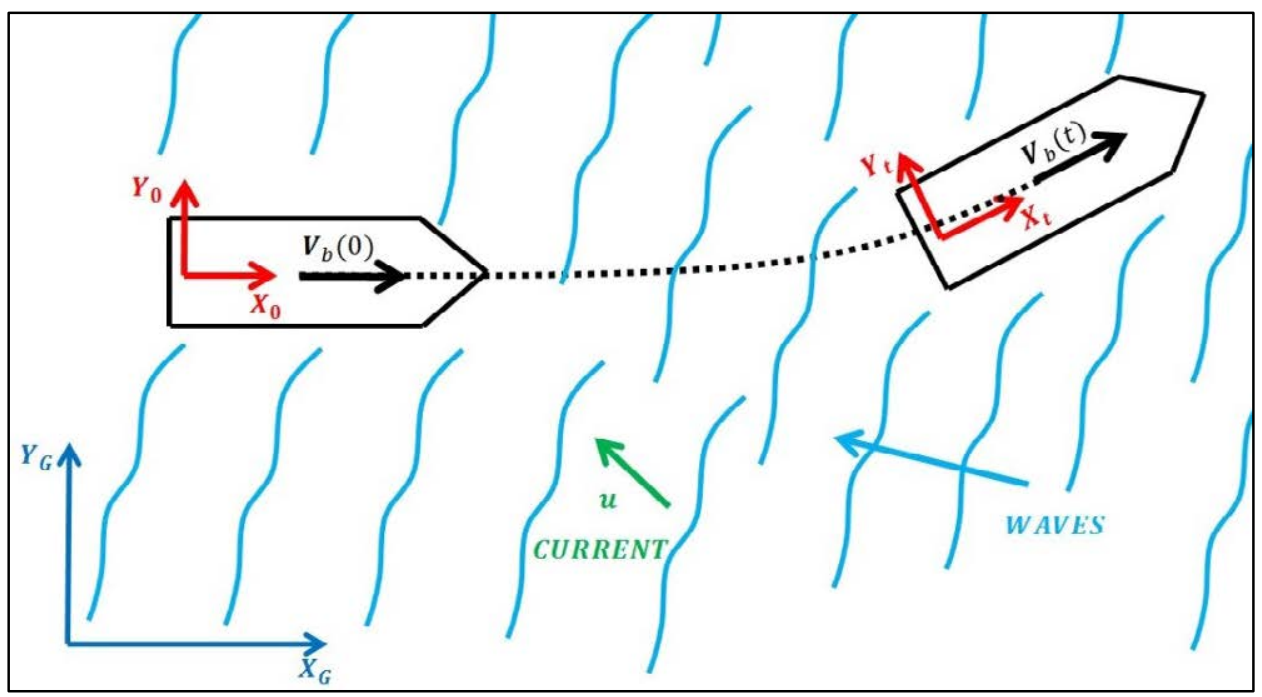

Figure 2: Global and local frame of reference.

\subsubsection{Flow linearization}

The previous governing equations have been obtained under the assumption that $\boldsymbol{v}_{\phi} \sim O(1)$. Then, the free surface boundary conditions can be written as follows:

$$
\begin{array}{ll}
\frac{\partial \phi}{\partial \mathrm{t}}+\left(\boldsymbol{U}_{b}+\nabla_{\mathrm{h}} \phi\right) \cdot \nabla_{\mathrm{h}} \phi-\frac{1}{2} \nabla_{\mathrm{h}} \phi \cdot \nabla_{\mathrm{h}} \phi+\nabla_{\mathrm{h}} \psi \cdot \nabla_{\mathrm{h}} \phi+\frac{P_{f s}}{\rho}+g \eta=0 & \text { on } z=0, \\
\frac{\partial \eta}{\partial t}+\left(\boldsymbol{U}_{b}+\nabla_{\mathrm{h}} \phi\right) \cdot \nabla_{\mathrm{h}} \eta+\nabla_{\mathrm{h}} \phi \cdot \nabla_{\mathrm{h}} \zeta-\frac{\partial \phi}{\partial \mathrm{z}}=0 & \text { on } z=0,
\end{array}
$$

where $\boldsymbol{U}_{b}+\nabla_{h} \phi$ represents the convective velocity. Since it depends on $\nabla_{h} \phi$, it must be updated every time step to account for its variations. While retaining this assumption allows for simulating transient flows, linearization of the convective velocity is a reasonable practice in many cases. Two commonly used linearizations are described next (see Figure 3).

\subsubsection{Neumann-Kelvin linearization}

The Neumann-Kelvin linearization assumes that $\nabla_{h} \phi \sim O(\epsilon)$. This assumption is usually made for slender bodies whose perturbation of the flow field is small compared to the apparent velocity between the moving body and the water. Then the convective velocity becomes the apparent velocity $\boldsymbol{U}_{b}(\boldsymbol{x})=$ $\boldsymbol{u}-\boldsymbol{V}_{b}(\boldsymbol{x})$. Hence the first-order free surface boundary conditions become:

$$
\begin{array}{ll}
\frac{\partial \phi}{\partial \mathrm{t}}+\boldsymbol{U}_{b} \cdot \nabla_{\mathrm{h}} \phi+\frac{P_{f s}}{\rho}+g \eta=0 & \text { on } z=0, \\
\frac{\partial \eta}{\partial t}+\boldsymbol{U}_{b} \cdot \nabla_{\mathrm{h}} \eta-\frac{\partial \phi}{\partial \mathrm{z}}=0 & \text { on } z=0,
\end{array}
$$


and the pressure induced by diffracted and radiated waves at point $\mathrm{P}$ is:

$$
P_{p}=-\rho\left(\frac{\partial \phi}{\partial \mathrm{t}}+\boldsymbol{U}_{b} \cdot \nabla_{\mathrm{h}} \phi\right)
$$

\subsubsection{Double body linearization}

Double body linearization assumes that $\nabla_{\mathrm{h}} \phi \sim O(1)$, but it can be split into two terms: $\nabla_{\mathrm{h}} \phi=$ $\nabla_{\mathrm{h}} \phi^{D B}+\nabla_{\mathrm{h}} \phi^{*}$, where $\nabla_{\mathrm{h}} \phi^{D B}$ represents the flow field when the free surface is substituted by a wall (equivalent to a symmetric case using a double body). Then $\nabla_{\mathrm{h}} \phi$ is approximated as $\nabla_{\mathrm{h}} \phi^{D B}$ perturbed by $\nabla_{\mathrm{h}} \phi^{*}$, which means $\nabla_{\mathrm{h}} \phi^{D B} \sim O(1)$ and $\nabla_{\mathrm{h}} \phi^{*} \sim O(\epsilon)$. Then the first-order free surface boundary conditions become:

$$
\begin{array}{rlr}
\frac{\partial \phi}{\partial \mathrm{t}}+\left(\boldsymbol{U}_{b}+\nabla_{\mathrm{h}} \phi^{D B}\right) \cdot \nabla_{\mathrm{h}} \phi-\frac{1}{2} \nabla_{\mathrm{h}} \phi^{D B} \nabla_{\mathrm{h}} \phi^{D B} & \\
+\nabla_{\mathrm{h}} \phi^{D B} \cdot \nabla_{\mathrm{h}} \psi+\frac{P_{f s}}{\rho}+g \eta=0 & \text { on } z=0, \\
\frac{\partial \eta}{\partial t}+\left(\boldsymbol{U}_{b}+\nabla_{\mathrm{h}} \phi^{D B}\right) \cdot \nabla_{\mathrm{h}} \eta+\nabla_{\mathrm{h}} \phi^{D B} \cdot \nabla_{\mathrm{h}} \zeta-\frac{\partial \phi}{\partial \mathrm{z}}=0 & \text { on } z=0,
\end{array}
$$

and the pressure induced by diffracted and radiated waves at point $\mathrm{P}$ is:

$$
P_{p}=-\rho\left(\frac{\partial \phi}{\partial t}+\left(\boldsymbol{U}_{b}+\nabla_{\mathrm{h}} \phi^{D B}\right) \cdot \nabla_{\mathrm{h}} \phi-\frac{1}{2} \nabla_{\mathrm{h}} \phi^{D B} \nabla_{\mathrm{h}} \phi^{D B}+\nabla_{\mathrm{h}} \phi^{D B} \cdot \nabla_{\mathrm{h}} \psi\right) \text {. }
$$

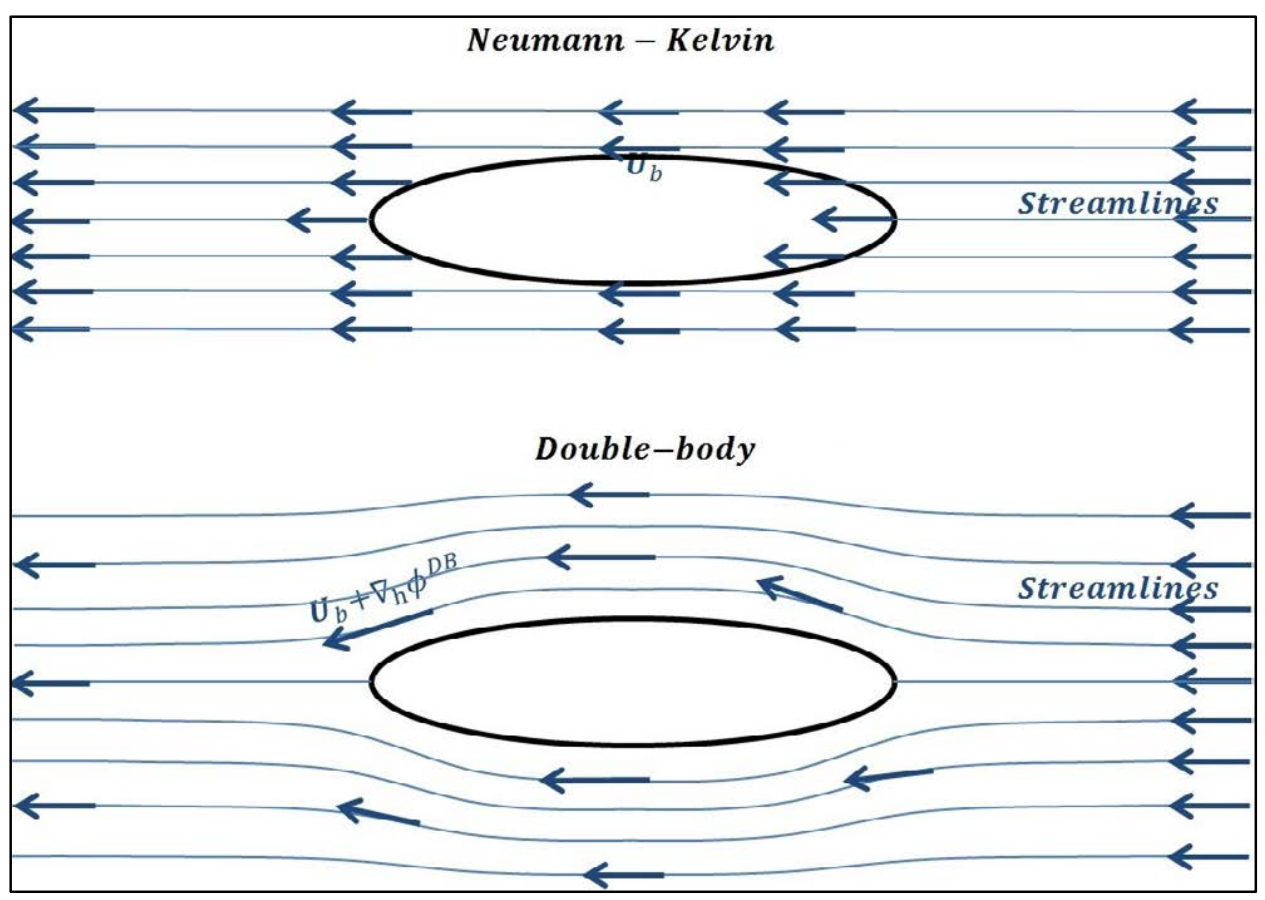

Figure 3: Free surface base flow based for Neumann-Kelvin (up) and double-body (down) linearization concepts.

\subsubsection{Second-order pressure correction over body}

A second-order correction on the pressure calculation is proposed in order to better estimate hydrodynamic loads. The main idea is to retain second-order terms depending on the first-order 
solution. Although this will not provide a full second-order solution, it will retain the terms contributing to mean drift loads (when $\boldsymbol{U}_{b}=0$ ), and added resistance in waves (when $\boldsymbol{U}_{b} \neq 0$ ).

The pressure at any point within the fluid is given by:

$$
P_{p}=-\rho\left(\frac{\partial \varphi}{\partial t}+\boldsymbol{U}_{b} \cdot \nabla_{\mathrm{h}} \varphi+\frac{1}{2} \nabla \varphi \cdot \nabla \varphi+g z_{p}\right)
$$

Introducing the velocity potential decomposition we obtain:

$$
\begin{aligned}
& P_{p}=P_{p}^{I}+P_{p}^{D R}+P_{p}^{c(2)}-\rho g z_{p}, \\
& P_{p}^{I}=-\rho\left(\frac{\partial \psi}{\partial t}+\boldsymbol{U}_{b} \cdot \nabla_{\mathrm{h}} \psi\right), \\
& P_{p}^{D R}=-\rho\left(\frac{\partial \phi}{\partial t}+\left(\boldsymbol{U}_{b}+\nabla_{\mathrm{h}} \phi+\nabla_{\mathrm{h}} \psi\right) \cdot \nabla_{\mathrm{h}} \phi-\frac{1}{2} \nabla_{\mathrm{h}} \phi \cdot \nabla_{\mathrm{h}} \phi\right), \\
& P_{p}^{c(2)}=-\rho \boldsymbol{r}_{p}^{1} \cdot \nabla\left(P_{p}^{I}+P_{p}^{D R}\right)-\rho\left(\frac{1}{2} \phi_{z}^{2}+\phi_{z} \psi_{z}+\frac{1}{2} \nabla \psi \cdot \nabla \psi\right),
\end{aligned}
$$

where $\boldsymbol{r}_{p}^{1}$ is the first-order displacement of a point $\mathrm{P}$ over the body surface, $P_{p}^{I}$ is the first-order incident

wave pressure, $P_{p}^{D R}$ is the first-order wave diffraction-radiation pressure, and $P_{p}^{c(2)}$ is the pressure correction term containing all the second-order terms depending on the first-order solution.

\subsubsection{Neumann-Kelvin}

When using the Newman-Kelvin linearization, $\nabla_{\mathrm{h}} \phi \sim O(\epsilon)$ is assumed. Hence, the pressure due to diffraction-radiation and the second-order pressure correction are:

$$
\begin{aligned}
& P_{p}^{D R}=-\rho\left(\frac{\partial \phi}{\partial t}+\boldsymbol{U}_{b} \cdot \nabla_{\mathrm{h}} \phi\right) \\
& P_{p}^{c(2)}=-\rho \boldsymbol{r}_{p}^{1} \cdot \nabla\left(P_{p}^{I}+P_{p}^{D R}\right)-\rho \frac{1}{2}(\nabla \phi+\nabla \psi)^{2} .
\end{aligned}
$$

\subsubsection{Double-body}

When using the Double-body linearization, it is assumed that $\nabla_{\mathrm{h}} \phi=\nabla_{\mathrm{h}} \phi^{D B}+\nabla_{\mathrm{h}} \phi^{*}$, where $\nabla_{\mathrm{h}} \phi^{D B} \sim O(1)$ and $\nabla_{\mathrm{h}} \phi^{*} \sim O(\epsilon)$. Hence, the pressure due to diffraction-radiation and the second-order pressure correction are:

$$
\begin{aligned}
& P_{p}^{D R}=-\rho\left(\frac{\partial \phi}{\partial t}+\left(\boldsymbol{U}_{b}+\nabla_{\mathrm{h}} \phi^{D B}\right) \cdot \nabla_{\mathrm{h}} \phi-\frac{1}{2} \nabla_{\mathrm{h}} \phi^{D B} \nabla_{\mathrm{h}} \phi^{D B}+\nabla_{\mathrm{h}} \phi^{D B} \cdot \nabla_{\mathrm{h}} \psi\right) \\
& P_{p}^{c(2)}=-\rho \boldsymbol{r}_{p}^{1} \cdot \nabla\left(P_{p}^{I}+P_{p}^{D R}\right)-\rho\left(\frac{1}{2}\left(\nabla_{\mathrm{h}} \phi^{*}+\nabla_{\mathrm{h}} \psi\right)^{2}+\frac{1}{2}\left(\phi_{\mathrm{z}}+\psi_{z}\right)^{2}\right) .
\end{aligned}
$$

\subsubsection{Second-order pressure correction on waterline}

When obtaining hydrodynamic forces and moments acting on a floating body, the first-order approximation integrates the pressure over the body surface below the mean waterline $(\mathrm{z}=0)$. However, 
the movements of the body and the free surface elevation around the body modify the wetted surface corresponding to the body in state of equilibrium and with unperturbed free surface. In order to take into account the contribution of the actual wetted body surface, a second-order correction is mandatory. In order to obtain such a correction, a linear pressure variation will be considered from the waterline of the body to the free surface. This pressure distribution will equal the pressure at the free surface and the pressure at the waterline. Figure 4 shows the concept of the pressure correction at the waterline area.

$$
\begin{aligned}
& P_{p}^{w l}=P_{p}^{I}+P_{p}^{D R}-\rho g \mathrm{r}_{\mathrm{pz}}, \\
& P_{p}^{w l(2)}(z)=P_{p}^{w l}-\left(P_{p}^{w l}-P_{p}^{f s}\right) \frac{z}{\xi-\mathrm{r}_{\mathrm{pz}}},
\end{aligned}
$$

where $P_{p}^{w l}$ is the first-order pressure at $\mathrm{z}=0$, and $P_{p}^{w l(2)}$ is the vertical pressure distribution in the water line area for second order correction.

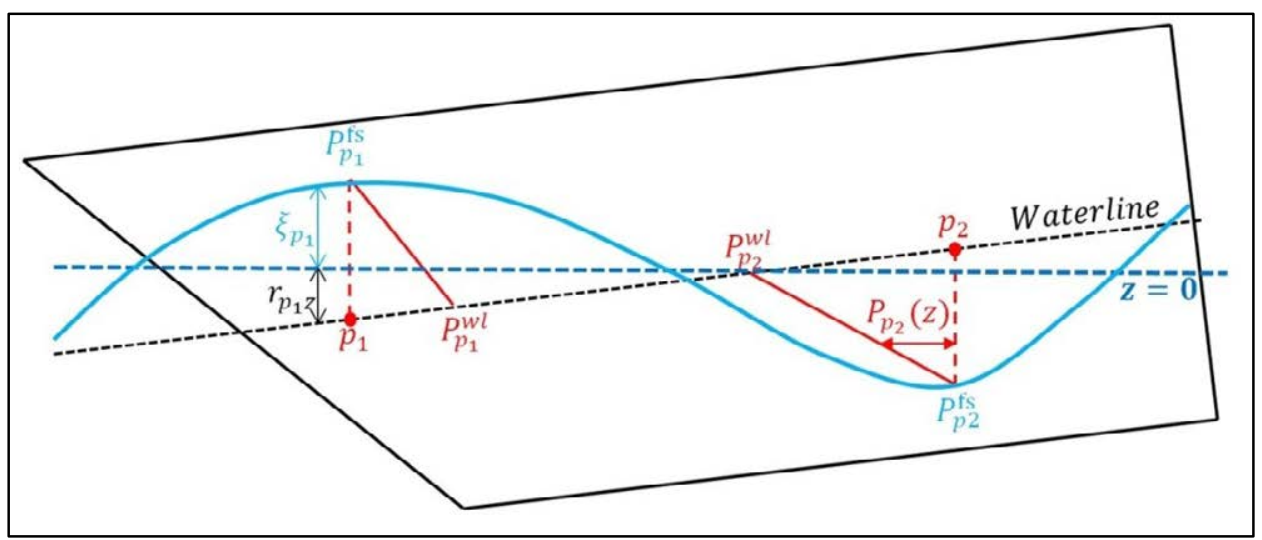

Figure 4: Pressure estimation at the waterline area.

\subsection{Numerical models for wave resistance problems}

\subsubsection{Glossary}

$\Omega \quad$ Fluid domain

$\Omega^{\mathrm{e}} \quad$ Fluid domain within element $\mathrm{e}$

$\phi \quad$ Diffraction-radiation velocity potential

$\eta \quad$ Diffraction-radiation wave elevation

$P_{f s} \quad$ Free surface pressure

$\boldsymbol{U} \quad$ Free surface convective velocity

$\overline{\bar{I}} \quad$ Identity matrix

$\overline{\overline{\mathbf{W}}} \quad$ Streamline convective matrix

$\overline{\overline{\mathbf{L}}}^{*} \quad$ Modified Laplacian FEM matrix

$\mathbf{b}^{Z_{0}} \quad$ FEM Dirichlet free surface boundary condition

$\mathbf{b}^{B} \quad$ FEM Neumann body boundary condition 

$\mathbf{b}^{Z_{H}} \quad$ FEM Neumann bottom boundary condition
$\Delta t \quad$ Time step
$\partial_{L} \quad$ Differential operator for first derivative along streamline
$\theta \quad$ Value between 0 and 1 .

\subsubsection{Introduction}

Section 3.2 presents the numerical schemes to cope with the governing equations presented in the previous section. Especially important are the numerical schemes used to solve the free surface boundary conditions, key point of the wave problem. Two different schemes are used: the first one is based on streamline integration; the second one is a stabilized Finite Element based on the streamlineupwind Petrov-Galerkin method. Both schemes are capable of coping with unstructured meshes.

\subsubsection{Stream line integration}

The first-order free surface boundary conditions can be written in a general form as follows:

$$
\begin{array}{ll}
\frac{\partial \phi}{\partial \mathrm{t}}+\boldsymbol{U} \cdot \nabla_{\mathrm{h}} \phi+\frac{P_{f s}}{\rho}+g \eta+R=0 & \text { on } z=0, \\
\frac{\partial \eta}{\partial t}+\boldsymbol{U} \cdot \nabla_{\mathrm{h}} \eta-\frac{\partial \phi}{\partial \mathrm{z}}+\mathrm{S}=0 & \text { on } z=0,
\end{array}
$$

where $R$ and $S$ represent remaining terms depending on the linearization used. The numerical schemes adopted for solving the kinematic-dynamic free surface boundary conditions are based on AdamsBashforth-Moulton schemes, using an explicit scheme for the kinematic condition, and an implicit one for the dynamic condition. Then $\phi^{n+1}$ is imposed as a Dirichlet boundary condition. The schemes read as follows:

$$
\begin{array}{ll}
\phi^{n+1}+\Delta \mathrm{t}\left(\boldsymbol{U} \cdot \nabla_{\mathrm{h}} \phi\right)^{n+1}=\phi^{n}-\Delta \mathrm{t}\left(\frac{1}{\rho} P_{f s}^{n+1}-g \eta^{n+1}-R^{n+1}\right) & \text { on } z=0, \\
\eta^{n+1}=\eta^{n}-\Delta \mathrm{t}\left(\boldsymbol{U} \cdot \nabla_{\mathrm{h}} \eta\right)^{n}+\Delta \mathrm{t}\left(\phi_{z}^{n}-\mathrm{S}^{\mathrm{n}}\right) & \text { on } z=0,
\end{array}
$$

where $\boldsymbol{U}$ is the convective velocity. The convective term is obtained by differentiating along streamlines:

$$
\begin{aligned}
& \left(\boldsymbol{U} \cdot \nabla_{\mathrm{h}} \phi\right)^{n+1}=|\boldsymbol{U}|^{n+1} \partial_{L} \phi^{n+1}, \\
& \left(\boldsymbol{U} \cdot \nabla_{\mathrm{h}} \eta\right)^{n}=|\boldsymbol{U}|^{n} \partial_{L} \eta^{n},
\end{aligned}
$$

where $\partial_{L}$ denotes the derivative along the streamline. This streamline derivative is estimated using a two points upstream and one point downstream differential operator. Figure 5 shows the tracing of the streamline at node $\mathrm{C}$. The left (-1) and forward left (-2) points are upstream points, while the right (1) point corresponds to the downstream point. The values of the scattered velocity potential $\phi$ and 
scattered free surface elevation $\eta$ at points $-1,-2$, and 1 are obtained by linear interpolation between the nodes of the edges where they lie on. Then the streamline differential operator reads as:

$$
\begin{aligned}
& \partial_{L} \phi_{0}=w_{1} \phi_{1}+w_{0} \phi_{0}+w_{-1} \phi_{-1}+w_{-2} \phi_{-2}, \\
& \partial_{L} \eta_{0}=w_{1} \eta_{1}+w_{0} \eta_{0}+w_{-1} \eta_{-1}+w_{-2} \eta_{-2},
\end{aligned}
$$

where $\phi_{1}, \phi_{-1}, \phi_{-2}$ are interpolated between $\left(\phi_{1 a}, \phi_{1 b}\right),\left(\phi_{-1 a}, \phi_{-1 b}\right)$, and $\left(\phi_{-2 a}, \phi_{-2 b}\right)$ respectively. In matrix form:

$$
\begin{array}{ll}
\left(\overline{\overline{\boldsymbol{I}}}+\Delta \mathrm{t} \overline{\overline{\mathbf{W}}}^{\boldsymbol{n}+\boldsymbol{1}}\right) \boldsymbol{\phi}^{n+1}=\overline{\overline{\boldsymbol{I}}}\left(\boldsymbol{\phi}^{n}-\Delta \mathrm{t}\left(\frac{1}{\rho} \boldsymbol{P}_{f s}^{n+1}-g \boldsymbol{\eta}^{n+1}-\boldsymbol{R}^{n+1}\right)\right) & \text { on } z=0, \\
\overline{\overline{\boldsymbol{I}}} \boldsymbol{\eta}^{n+1}=\left(\overline{\overline{\boldsymbol{I}}}-\Delta \mathrm{t} \overline{\overline{\mathbf{W}}}^{\boldsymbol{n}}\right) \boldsymbol{\eta}^{n}+\Delta \mathrm{t}\left(\boldsymbol{\phi}_{z}^{n}-\boldsymbol{S}^{\mathrm{n}}\right) & \text { on } z=0,
\end{array}
$$

where $\overline{\overline{\mathbf{W}}}$ is the streamline convective matrix, and $\overline{\overline{\boldsymbol{I}}}$ is the identity matrix. The stencils are obtained using Taylor series expansion to tailor a second-order finite difference scheme along the streamline. The corresponding system of equations is:

$$
\begin{aligned}
& w_{1}+w_{0}+w_{-1}+w_{-2}=0, \\
& w_{1} \Delta x_{1}-w_{-1} \Delta x_{-1}-w_{-2} \Delta x_{-2}=1, \\
& w_{1} \frac{\Delta x_{1}^{2}}{2}+w_{-1} \frac{\Delta x_{-1}^{2}}{2}+w_{-2} \frac{\Delta x_{-2}^{2}}{2}=0, \\
& w_{1} \frac{\Delta x_{1}^{3}}{6}-w_{-1} \frac{\Delta x_{-1}^{3}}{6}-w_{-2} \frac{\Delta x_{-2}^{3}}{6}=0 .
\end{aligned}
$$

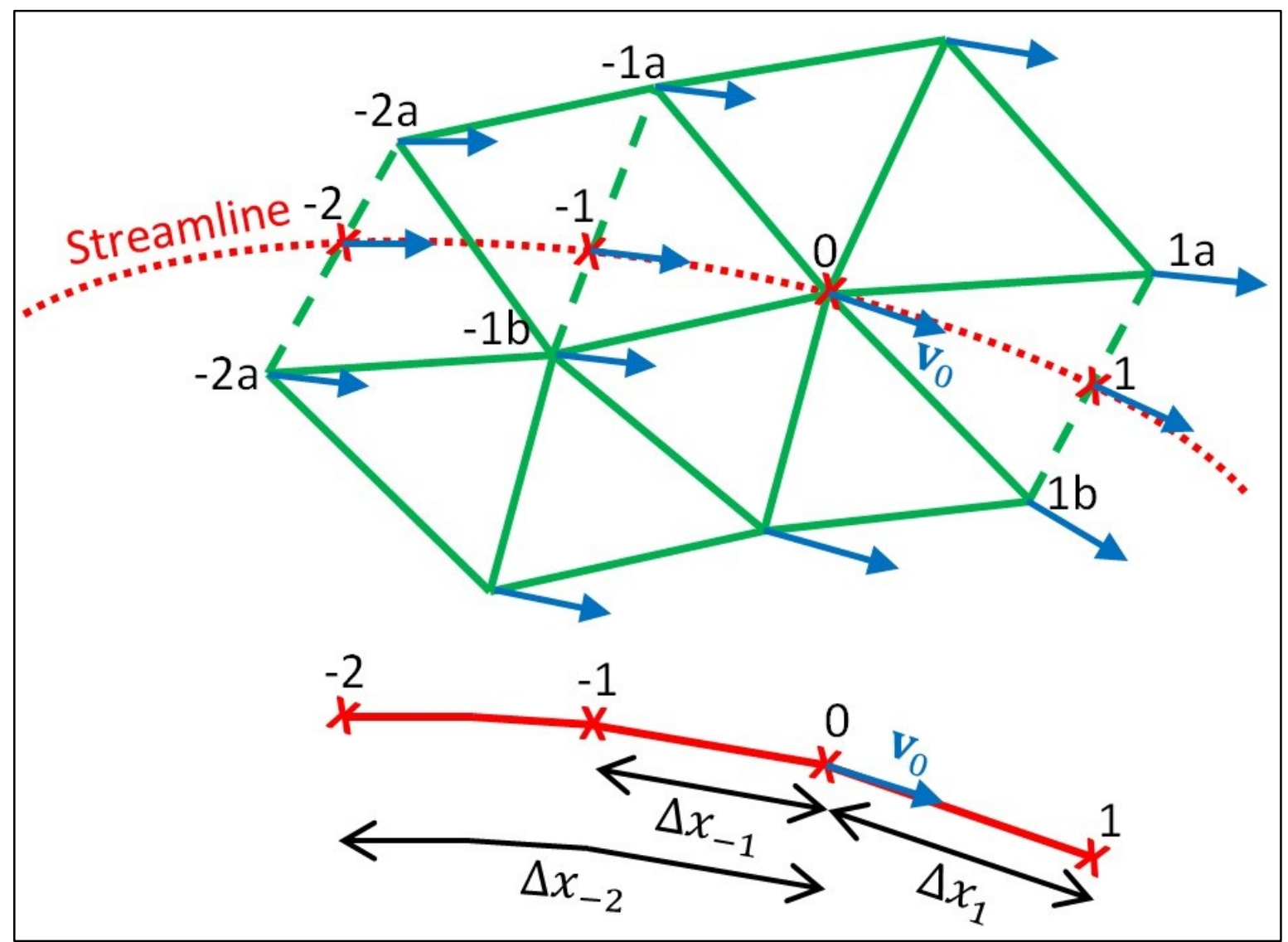

Figure 5: Streamline discretization 
The associated matrix form to the finite element formulation for the governing equations is:

$\overline{\overline{\mathbf{L}^{*}}} \boldsymbol{\phi}=\mathbf{b}^{Z_{0}}+\mathbf{b}^{B}+\mathbf{b}^{R}$,

where $\overline{\overline{\mathbf{L}^{*}}}$ is the standard Laplacian matrix modified to account for the left hand side of Eq.(3-58), $\mathbf{b}^{Z_{0}}$ is a vector accounting for the right hand side of Eq. (3-58), and $\mathbf{b}^{B}$ and $\mathbf{b}^{R}$ are the vectors resulting of integrating the corresponding boundary condition terms.

\subsubsection{Streamline-Upwind Petrov-Galerkin (SUPG) formulation}

Alternatively, a SUPG stabilization scheme is also developed for the integration of the free surface boundary conditions. Both, the dynamic and kinematic boundary conditions can be seen as a convective-transport equation:

$\frac{\partial \chi}{\partial \mathrm{t}}+\boldsymbol{U} \cdot \nabla_{\mathrm{h}} \chi+Q=0$.

The standard Galerkin finite element formulation leads to the following system of equations:

$\forall i \int_{\Omega} N^{i}\left(N^{j} \frac{\partial \chi_{j}}{\partial \mathrm{t}}+\boldsymbol{U} \cdot \nabla_{\mathrm{h}} N^{j} \chi_{j}+N^{j} Q_{j}\right) d \sigma=0$,

where $N^{i}$ are the standard piecewise linear function. The above equations can be discretized in time as follows:

$$
\begin{gathered}
\forall i \int_{\Omega} N^{i} N^{j} \frac{\chi_{j}^{n+1}-\chi_{j}^{n}}{\Delta t} d \sigma+\int_{\Omega} N^{i}\left(\mathbf{U}^{n+\theta} \cdot \nabla_{\mathrm{h}} N^{j}\right) \chi_{j}^{n+\theta} d \sigma+\int_{\Omega} N^{i} N^{j} Q_{j}^{n+\theta} d \sigma \\
=0,
\end{gathered}
$$

where $A^{n+\theta}=(1-\theta) A^{n}+\theta A^{n+1}$. It is well known that the numerical solution of the previous equation may show spurious oscillations [38,39]. The SUPG formulation [40] is used to introduced the necessary stabilization. Then, the resulting scheme is:

$$
\begin{aligned}
& \forall i\left[\int_{\Omega} N^{i} N^{j} d \sigma\right] \frac{\chi_{j}^{n+1}-\chi_{j}^{n}}{\Delta t}+\left[\int_{\Omega} N^{i}\left(\left(\boldsymbol{U}^{e}\right)^{n+\theta} \cdot \nabla_{h} N^{j}\right) d \sigma\right] \chi_{j}^{n+\theta} \\
& +\left[\int_{\Omega} N^{i} N^{j} d \sigma\right] Q_{j}^{n+\theta}+\left[\sum_{e} \frac{h_{e}}{2\left|\left(\widehat{\boldsymbol{U}^{e}}\right)^{n+\theta}\right|} \int_{\Omega^{e}}\left(\left(\boldsymbol{U}^{e}\right)^{n+\theta} \cdot \nabla_{h} N^{i}\right) N^{j} d \sigma\right] \frac{\chi_{j}^{n+1}-\chi_{j}^{n}}{\Delta t} \\
& +\left[\sum_{e} \frac{h_{e}}{2 \mid\left(\widehat{\boldsymbol{U}}^{e}\right)^{n+\theta \mid}} \int_{\Omega^{e}}\left(\left(\boldsymbol{U}^{e}\right)^{n+\theta} \cdot \nabla_{h} N^{i}\right)\left(\left(\boldsymbol{U}^{e}\right)^{n+\theta} \cdot \nabla_{h} N^{j}\right) d \sigma\right] \chi_{j}^{n+\theta} \\
& +\left[\sum_{e} \frac{h_{e}}{2 \mid\left(\widehat{\boldsymbol{U}^{e}}\right)^{n+\theta \mid}} \int_{\Omega^{e}}\left(\left(\boldsymbol{U}^{e}\right)^{n+\theta} \cdot \nabla_{h} N^{i}\right) N^{j} d \sigma\right] Q_{j}^{n+\theta}=0,
\end{aligned}
$$


where the convective velocity within the element is approximated by $\left(U^{e}\right)^{n+\theta}=\sum_{k_{e}} N^{k_{e}} U_{k_{e}}^{n+\theta}$. Reordering terms:

$$
\begin{aligned}
& \forall i\left[\int_{\Omega} N^{i} N^{j} d \sigma+\sum_{e} \frac{h_{e}}{2\left|\left(\widehat{\boldsymbol{U}^{e}}\right)^{n+\theta}\right|} \int_{\Omega^{e}}\left(\left(\boldsymbol{U}^{e}\right)^{n+\theta} \cdot \nabla_{h} N^{i}\right) N^{j} d \sigma\right]\left(\chi_{j}^{n+1}-\chi_{j}^{n}\right) \\
& +\Delta t\left[\int_{\Omega} N^{i}\left(\left(\boldsymbol{U}^{e}\right)^{n+\theta} \cdot \nabla_{h} N^{j}\right) d \sigma\right] \chi_{j}^{n+\theta} \\
& +\Delta t\left[\sum_{e} \frac{h_{e}}{2 \mid\left(\widehat{\boldsymbol{U}}^{e}\right)^{n+\theta \mid}} \int_{\Omega^{e}}\left(\left(\boldsymbol{U}^{e}\right)^{n+\theta} \cdot \nabla_{h} N^{i}\right)\left(\left(\boldsymbol{U}^{e}\right)^{n+\theta} \cdot \nabla_{h} N^{j}\right) d \sigma\right] \chi_{j}^{n+\theta} \\
& +\Delta t\left[\int_{\Omega} N^{i} N^{j} d \sigma+\sum_{e} \frac{h_{e}}{2\left|\left(\widehat{\boldsymbol{U}}^{e}\right)^{n+\theta}\right|} \int_{\Omega^{e}}\left(\left(\boldsymbol{U}^{e}\right)^{n+\theta} \cdot \nabla_{h} N^{i}\right) N^{j} d \sigma\right] Q_{j}^{n+\theta}=0 .
\end{aligned}
$$

In matrix form:

$$
\begin{aligned}
& \left(\overline{\overline{\boldsymbol{M}}}+\overline{\overline{\boldsymbol{M}}}_{\text {supg }}^{n+\theta}\right) \chi^{\mathrm{n}+1}+\Delta \mathrm{t}\left(\overline{\overline{\boldsymbol{C}}}^{n+\theta}+\overline{\overline{\boldsymbol{C}}}_{\text {supg }}^{n+\theta}\right) \theta \chi^{\mathrm{n}+1}= \\
& \left(\overline{\bar{M}}+\overline{\overline{\boldsymbol{M}}}_{\text {supg }}^{n+\theta}\right) \chi^{\mathrm{n}}-\Delta \mathrm{t}\left(\overline{\overline{\boldsymbol{C}}}^{n+\theta}+\overline{\overline{\boldsymbol{C}}}_{\text {supg }}^{n+\theta}\right)(1-\theta) \chi^{\mathrm{n}}-\left(\overline{\overline{\boldsymbol{M}}}+\overline{\overline{\boldsymbol{M}}}_{\text {supg }}^{n+\theta}\right) \boldsymbol{Q}^{n+\theta},
\end{aligned}
$$

where:

$$
\begin{aligned}
& \overline{\overline{\boldsymbol{M}}}=\int_{\Omega} N^{i} N^{j} d \sigma \\
& \overline{\overline{\boldsymbol{M}}}_{\text {supg }}^{\mathrm{n}+\theta}=\sum_{e}\left[\frac{h_{e}}{2\left|\left(\widehat{\boldsymbol{U}}^{e}\right)^{n+\theta}\right|_{\Omega^{\mathrm{e}}}}\left(\left(\boldsymbol{U}^{e}\right)^{n+\theta} \cdot \nabla_{h} N^{i}\right) N^{j} d \sigma\right] \\
& \overline{\overline{\boldsymbol{C}}}^{\mathrm{n}+\theta}=\sum_{e} \int_{\Omega^{e}} N^{i}\left(\boldsymbol{U}_{e}^{n+\theta} \cdot \nabla_{h} N^{j}\right), \\
& \overline{\overline{\boldsymbol{C}}}_{\text {supg }}^{\mathrm{n}+\theta}=\sum_{e}\left[\frac{h_{e}}{2 \mid\left(\widehat{\boldsymbol{U}}^{e}\right)^{n+\theta}} \int_{\Omega^{e}}\left(\left(\boldsymbol{U}^{e}\right)^{n+\theta} \cdot \nabla_{h} N^{i}\right)\left(\boldsymbol{U}_{e}^{n+\theta} \cdot \nabla_{h} N^{j}\right) d \sigma\right] .
\end{aligned}
$$

Likewise, the dynamic boundary condition becomes:

$$
\begin{gathered}
\left(\overline{\overline{\boldsymbol{M}}}+\overline{\overline{\boldsymbol{M}}}_{\text {supg }}^{n+\theta}\right) \boldsymbol{\phi}^{\mathrm{n}+1}+\boldsymbol{\Delta} \mathrm{t}\left(\overline{\overline{\boldsymbol{C}}}^{n+\theta}+\overline{\overline{\boldsymbol{C}}}_{\text {supg }}^{n+\theta}\right) \theta \boldsymbol{\phi}^{\mathrm{n}+1}= \\
\left(\overline{\overline{\boldsymbol{M}}}+\overline{\overline{\boldsymbol{M}}}_{\text {supg }}^{n+\theta}\right) \boldsymbol{\phi}^{\mathrm{n}}-\boldsymbol{\Delta \mathrm { t }}\left(\overline{\overline{\boldsymbol{C}}}^{n+\theta}+\overline{\overline{\boldsymbol{C}}}_{\text {supg }}^{n+\theta}\right)(1-\theta) \boldsymbol{\phi}^{\mathrm{n}} \\
-\left(\overline{\overline{\boldsymbol{M}}}+\overline{\overline{\boldsymbol{M}}}_{\text {supg }}^{n+\theta}\right)\left(\frac{1}{\rho} \boldsymbol{P}^{n+\theta}+g \boldsymbol{\eta}^{n+\theta}+\boldsymbol{R}^{n+\theta}\right) .
\end{gathered}
$$


And the kinematic boundary condition becomes:

$$
\begin{aligned}
\left(\overline{\overline{\boldsymbol{M}}}+\overline{\overline{\boldsymbol{M}}}_{\text {supg }}^{n+\theta}\right) \boldsymbol{\eta}^{\mathrm{n}+1}+ & \Delta \mathrm{t}\left(\overline{\overline{\boldsymbol{C}}}^{n+\theta}+\overline{\overline{\boldsymbol{C}}}_{\text {supg }}^{n+\theta}\right) \theta \boldsymbol{\eta}^{\mathrm{n}+1}= \\
& \left(\overline{\overline{\boldsymbol{M}}}+\overline{\overline{\boldsymbol{M}}}_{\text {supg }}^{n+\theta}\right) \boldsymbol{\eta}^{\mathrm{n}}-\boldsymbol{\Delta} \mathrm{t}\left(\overline{\overline{\boldsymbol{C}}}^{n+\theta}+\overline{\overline{\boldsymbol{C}}}_{\text {supg }}^{n+\theta}\right)(1-\theta) \boldsymbol{\eta}^{\mathrm{n}} \\
+ & \left(\overline{\overline{\boldsymbol{M}}}+\overline{\overline{\boldsymbol{M}}}_{\text {supg }}^{n+\theta}\right)\left(\frac{1}{\rho} \boldsymbol{\phi}_{z}^{n+\theta}-\boldsymbol{S}^{n+\theta}\right) .
\end{aligned}
$$

In this thesis, an explicit scheme $(\theta=0)$ is used for the kinematic condition, while an implicit scheme $(\theta=1)$ is used for the dynamic. The associated matrix form to the finite element formulation for the governing equations is:

$$
\overline{\mathbf{L}^{*}} \boldsymbol{\phi}=\mathbf{b}^{Z_{0}}+\mathbf{b}^{B}+\mathbf{b}^{R},
$$

where $\overline{\overline{\mathbf{L}^{*}}}$ is the standard Laplacian matrix modified to account for the left hand side of Eq. (3-69), $\mathbf{b}^{Z_{0}}$ is a vector accounting for the right hand side of Eq. (3-69), and $\mathbf{b}^{B}$ and $\mathbf{b}^{R}$ are the vectors resulting of integrating the corresponding boundary condition terms.

\subsection{Hydrodynamic loads on bodies for wave resistance problems}

\subsubsection{Glossary}

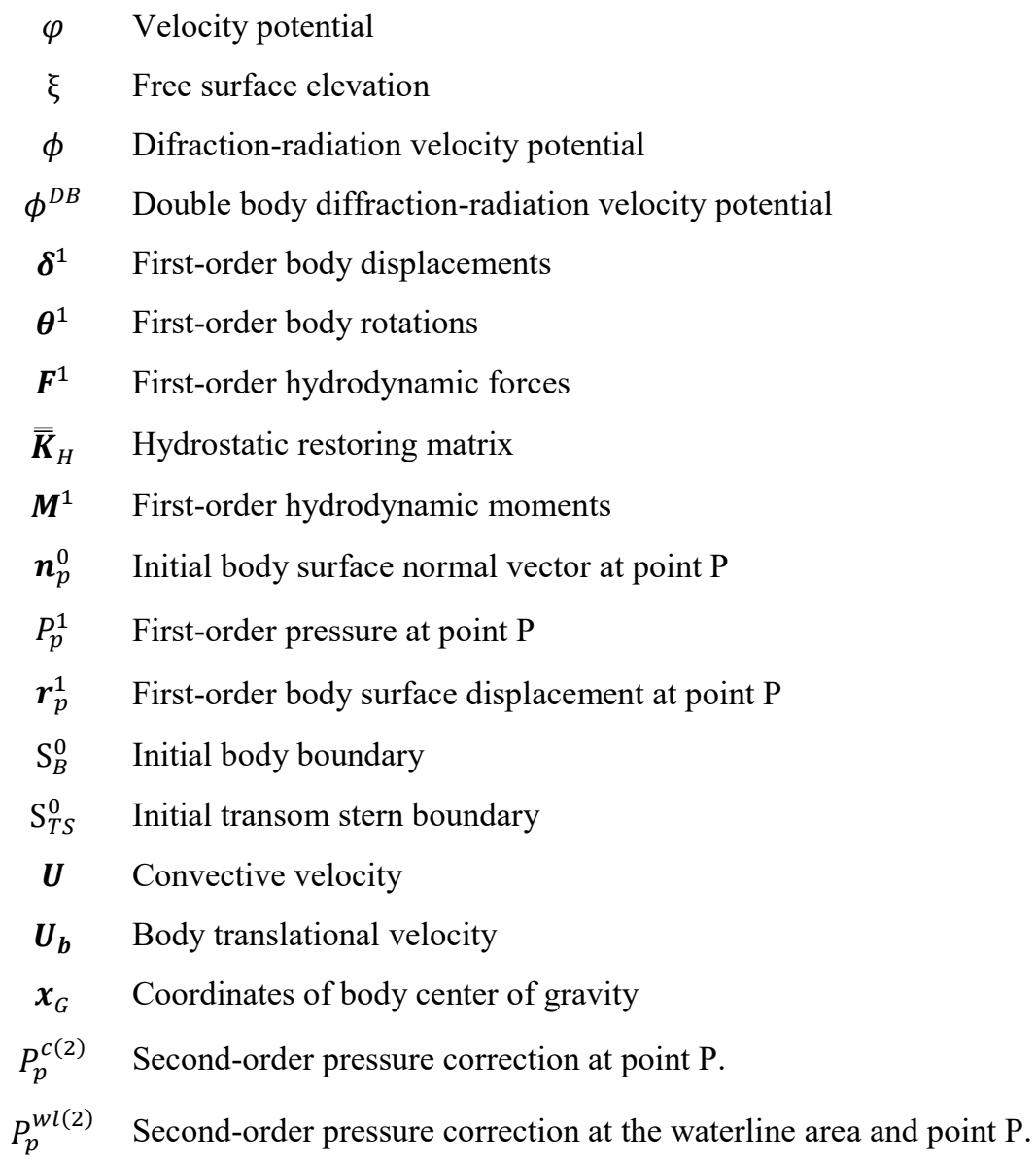




\subsubsection{Introduction}

The aim of section 3.3 is to obtain the hydrodynamic forces and moments on a floating body from direct pressure integration. These loads are split in hydrostatic and dynamic, obtained by integrating the hydrostatic and dynamic pressure respectively.

\subsubsection{First-order loads}

Hydrodynamic forces and moments are obtained from direct pressure integration over the body surface. The body gravity center will be used as a reference for body movements and moments acting on it. The resulting forces and moments are:

$$
\begin{aligned}
& \boldsymbol{F}^{1}=\int_{S_{B}^{0}} P_{p}^{1} \boldsymbol{n}_{p}^{0} d s-\int_{S_{T S}^{0}} P_{p}^{1} \boldsymbol{n}_{p}^{0} d s=\boldsymbol{F}_{H}^{0}+\boldsymbol{F}_{H}^{1}+\boldsymbol{F}_{D}^{1}, \\
& \boldsymbol{M}^{1}=\int_{\mathrm{S}_{B}^{0}} P_{p}^{1}\left(\boldsymbol{x}_{G}-\boldsymbol{x}_{p}\right) \times \boldsymbol{n}_{p}^{0} d s-\int_{\mathrm{S}_{T S}^{0}} P_{p}^{1}\left(\boldsymbol{x}_{G}-\boldsymbol{x}_{p}\right) \times \boldsymbol{n}_{p}^{0} d s=\boldsymbol{M}_{H}^{0}+\boldsymbol{M}_{H}^{1}+\boldsymbol{M}_{D}^{1},
\end{aligned}
$$

where sub-index H stands for hydrostatic loads, and D stands for dynamic loads.

\subsubsection{Hydrostatic loads}

The hydrostatic loads are split as follows:

$$
\begin{aligned}
& \boldsymbol{F}_{H}^{0}=-\int_{S_{B}^{0}} \rho g z \boldsymbol{n}_{p}^{0} d s=\rho g \forall, \\
& \boldsymbol{M}_{H}^{0}=-\int_{S_{B}^{0}} \rho g z \overrightarrow{\boldsymbol{G}^{0} \boldsymbol{P}^{0}} \times \boldsymbol{n}_{p}^{0} d s, \\
& \boldsymbol{F}_{H}^{1}=-\int_{S_{B}^{0}} \rho g r_{p z}^{1} \boldsymbol{n}_{p}^{0} d s, \\
& \boldsymbol{M}_{H}^{1}=-\int_{S_{B}^{0}} \rho g r_{p z}^{1} \overrightarrow{\boldsymbol{G}^{0} \boldsymbol{P}^{0}} \times \boldsymbol{n}_{p}^{0} d s,
\end{aligned}
$$

where $\forall$ is the body displacement. Hydrostatic forces and moments are obtained via the hydrostatic restoring matrix $\overline{\mathbf{K}}_{H}$ (defined in section 2.3.3):

$$
\left[\begin{array}{l}
\boldsymbol{F}_{H}^{1} \\
\boldsymbol{M}_{H}^{1}
\end{array}\right]=\overline{\overline{\boldsymbol{K}}}_{H}\left[\begin{array}{l}
\boldsymbol{\delta}^{1} \\
\boldsymbol{\theta}^{1}
\end{array}\right]
$$




\subsubsection{Dynamic loads}

The dynamic loads are computed as:

$$
\begin{aligned}
& \boldsymbol{F}_{D}^{1}=-\int_{\mathrm{S}_{B}^{0}} \rho\left(\frac{\partial \varphi}{\partial t}+\boldsymbol{U} \cdot \nabla_{\mathrm{h}} \varphi+Q\right) \boldsymbol{n}_{p}^{0} d s, \\
& \boldsymbol{M}_{D}^{1}=-\int_{\mathrm{S}_{B}^{0}} \rho\left(\frac{\partial \varphi}{\partial t}+\boldsymbol{U} \cdot \nabla_{\mathrm{h}} \varphi+Q\right) \overrightarrow{\boldsymbol{G}^{0} \boldsymbol{P}^{0}} \times \boldsymbol{n}_{p}^{0} d s,
\end{aligned}
$$

where $\boldsymbol{U}$ and Q depend on the flow approximation used (see Table 2).

Table 2: values of $\boldsymbol{U}$ and Q (see section 3.1.6)

\begin{tabular}{|c|c|c|}
\hline Flow type & $\boldsymbol{U}$ & $\boldsymbol{Q}$ \\
\hline Kelvin & $\boldsymbol{U}_{\boldsymbol{b}}$ & 0 \\
\hline Double body & $\boldsymbol{U}_{\boldsymbol{b}}+\nabla_{\mathrm{h}} \phi^{D B}$ & $-\frac{1}{2} \nabla_{\mathrm{h}} \phi^{D B} \nabla_{\mathrm{h}} \phi^{D B}$ \\
\hline Non-linear & $\boldsymbol{U}_{\boldsymbol{b}}+\nabla_{\mathrm{h}} \phi$ & $-\frac{1}{2} \nabla_{\mathrm{h}} \phi \cdot \nabla_{\mathrm{h}} \phi$ \\
\hline
\end{tabular}

\subsubsection{Second-order correction}

Second-order pressure correction leads to second-order correction loads. The latter contain the second-order effects depending on the first-order solution. Integrating the corresponding pressure:

$$
\begin{aligned}
& \boldsymbol{F}^{c(2)}=\int_{S_{B}} P_{p}^{c(2)} \boldsymbol{n}_{p}^{0} d s+\int_{\Gamma_{w l}} P_{p}^{w l(2)} \boldsymbol{n}_{p}^{0} / \sqrt{1-n_{p z}^{0}{ }^{2}} d l, \\
& \boldsymbol{M}^{c(2)}=\int_{S_{B}} P_{p}^{c(2)} \overrightarrow{\boldsymbol{G P}} \times \boldsymbol{n}_{p}^{0} d s+\int_{\Gamma_{w l}} P_{p}^{w l(2)} \overline{\boldsymbol{G P}} \times \boldsymbol{n}_{p}^{0} / \sqrt{1-n_{p z}^{02}} d l .
\end{aligned}
$$

A special meaning has the mean value of the correction loads. In the specific case where $\boldsymbol{U}=0$ and in the presence of waves, it represents the mean drift forces due to waves. And when $\boldsymbol{U} \neq 0$, they become the added resistance in waves.

\subsubsection{Other second-order loads}

Following the procedure carried out in section 2.3.4, the following second order correction terms depending on first order solution can be derived as well:

$$
\begin{aligned}
& \boldsymbol{F}_{H}^{2}=\boldsymbol{\theta}^{1} \times \boldsymbol{F}_{H}^{1}-\int_{S_{B}^{0}} \rho g\left(\overline{\overline{\boldsymbol{H}}} \overrightarrow{\boldsymbol{R}^{0} \boldsymbol{P}^{0}}\right)_{z} \boldsymbol{n}_{p}^{0} d s, \\
& \boldsymbol{M}_{H}^{2}=\boldsymbol{\theta}^{1} \times \boldsymbol{M}_{H}^{1}-\int_{S_{B}^{0}} \rho g\left(\overline{\overline{\boldsymbol{H}}} \overrightarrow{\boldsymbol{R}^{0} \boldsymbol{P}^{0}}\right)_{z} \overrightarrow{\boldsymbol{G}^{0} \boldsymbol{P}^{0}} \times \boldsymbol{n}_{p}^{0} d s . \\
& \boldsymbol{F}_{D}^{2}=\boldsymbol{\theta}^{1} \times \boldsymbol{F}_{D}^{1}, \\
& \boldsymbol{M}_{D}^{2}=\boldsymbol{\theta}^{1} \times \boldsymbol{M}_{D}^{1},
\end{aligned}
$$




\section{Chapter 4. BODY DYNAMICS SOLVER}

\subsection{Glossary}

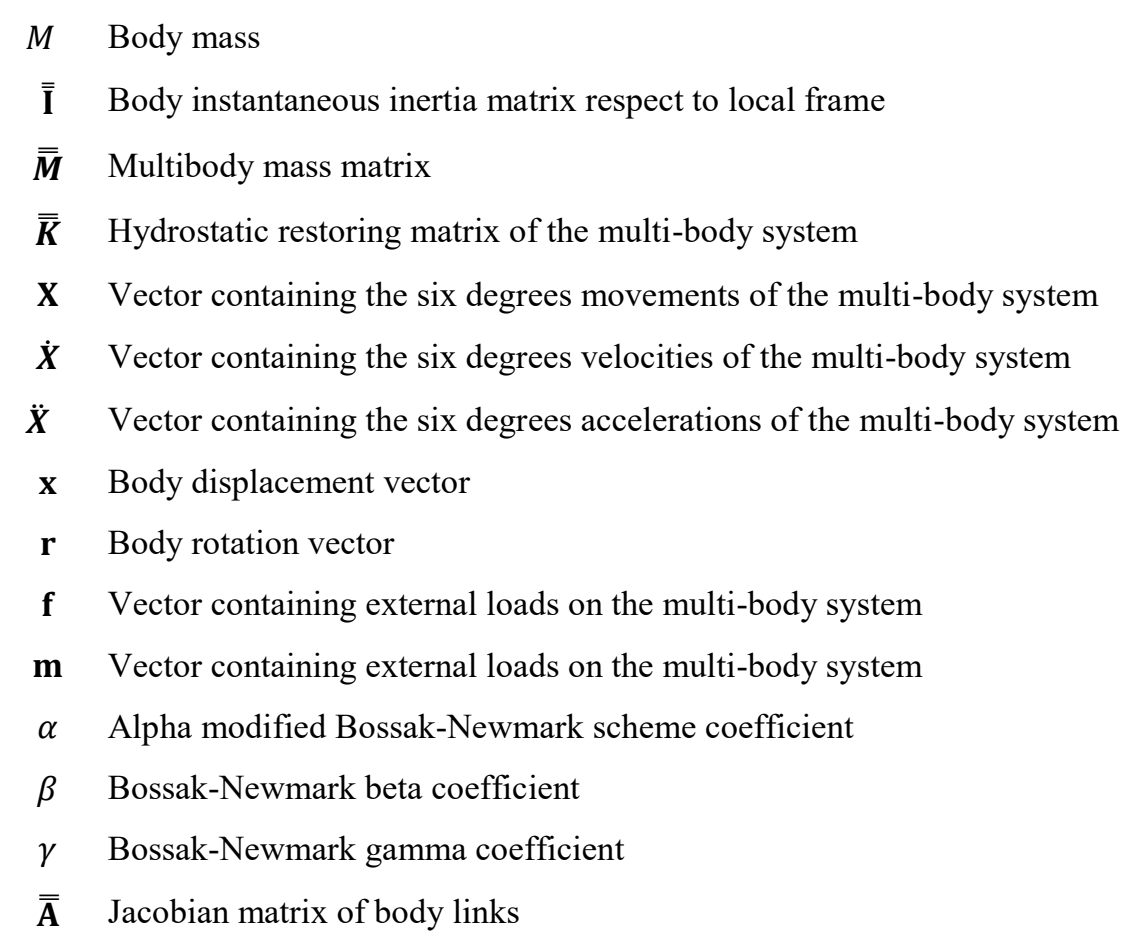

\subsection{Introduction}

This chapter presents a method to solve the rigid body dynamics equations of a multibody system whose bodies' motions may depend on each other. First, the body dynamic equations are presented, as well as the integration scheme used to solve them. Then, how to bring body links into the body dynamics (represented by kinematic constraints among degrees of freedom) by means of Lagrange multipliers is explained. Finally, the numerical schemes used for incorporating body links are described.

\subsection{Dynamic equations (small rotations)}

On one hand, by integrating the pressure over the bodies' surface, the external forces and moments are obtained. On the other hand, the body dynamics is given by the equation of motion:

$$
\overline{\bar{M}} \ddot{X}+\overline{\bar{K}} X=F \text {. }
$$

An implicit alpha modified Bossak-Newmark's algorithm [41] is used for integrating the body dynamics equations: 


$$
\begin{aligned}
& (1-\alpha) \mathbf{X}_{t t}^{n+1}+\alpha \mathbf{X}_{t t}^{n}=\overline{\bar{M}}^{-1}\left(\mathbf{F}^{n+1}-\overline{\bar{K}} \mathbf{X}^{n+1}\right), \\
& \mathbf{X}_{t}^{n+1}=\mathbf{X}_{t}^{n}+\Delta t\left[(1-\gamma) \mathbf{X}_{t t}^{n+1}+\gamma \mathbf{X}_{t t}^{n}\right], \\
& \mathbf{X}^{n+1}=\mathbf{X}^{n}+\Delta t \mathbf{X}_{t}^{n}+\frac{\Delta t^{2}}{2}\left[(1-2 \beta) \mathbf{X}_{t t}^{n+1}+2 \beta \mathbf{X}_{t t}^{n}\right]
\end{aligned}
$$

In this integration algorithm, $\alpha$ is the parameter controlling a numerical damping added in the integration. This damping creates a desirable stabilizing effect in the body dynamics integration. $\alpha$ is usually set between 0 (no damping) and -0.1. The parameters $\gamma$ and $\beta$ are calculated as $\gamma=0.5-\alpha$ and $\beta=0.5 \gamma+0.025 \alpha$. When $\alpha$ is set to zero, no dissipation is introduced and the scheme becomes the Bossak-Newmark with $\gamma=0.5$ and $\beta=0.25$, which is a well-known energy conserving scheme.

\subsection{Dynamic equations (large rotations)}

When large rotations are expected, the Euler equations are used to integrate the body dynamics. The accelerations of each body are solved in a local frame of reference attached to the body with origin in its gravity center, and whose axis are parallel to the global frame of reference (see Figure 6).

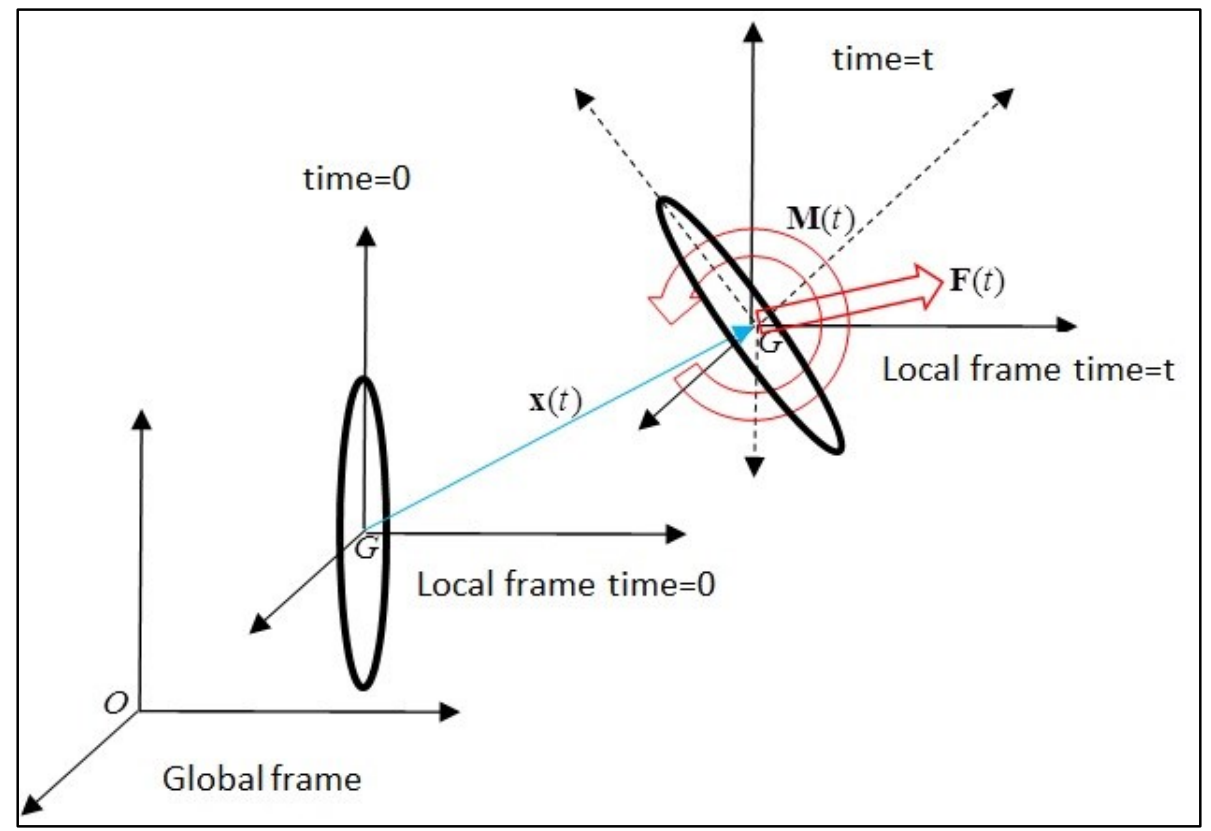

Figure 6: Global and local frame of reference used.

The dynamic equations for each body are the Newton and Euler equations in an inertial frame of reference:

$$
\begin{aligned}
& \frac{d\left(M_{i} \dot{\boldsymbol{x}}_{l}\right)}{d t}=M_{i} \ddot{\boldsymbol{x}}_{i}=\boldsymbol{f}_{i}, \\
& \frac{d\left(\overline{\overline{\boldsymbol{I}}}_{i} \dot{\boldsymbol{r}}_{l}\right)}{d t}=\overline{\overline{\boldsymbol{I}}}_{i} \ddot{\boldsymbol{r}}_{i}+\left(\frac{d \overline{\overline{\boldsymbol{I}}}_{i}}{d t}\right) \dot{\boldsymbol{r}}_{i}=\boldsymbol{m}_{i},
\end{aligned}
$$


where $\overline{\bar{I}}_{i}$ the instantaneous inertia tensor of body " $i$ " with respect to the local reference. Using the following equality: $\left(d \overline{\overline{\boldsymbol{I}}}_{i} / d t\right) \dot{\boldsymbol{r}}_{i}=\dot{\boldsymbol{r}}_{i} \wedge\left(\overline{\overline{\boldsymbol{I}}}_{i} \cdot \dot{\boldsymbol{r}}_{i}\right)$, the Euler equation becomes:

$$
\overline{\overline{\boldsymbol{I}}}_{i} \ddot{\boldsymbol{r}}_{i}=\boldsymbol{m}_{i}-\dot{\boldsymbol{r}}_{i} \wedge\left(\overline{\overline{\boldsymbol{I}}}_{i} \cdot \dot{\boldsymbol{r}}_{i}\right)
$$

It is important to remark that $\boldsymbol{f}_{i}$ and $\boldsymbol{m}_{i}$ must be evaluated in the local reference frame.

\subsection{Body-links}

\subsubsection{Lagrange multipliers}

Let a multibody system be defined by the dynamics equations:

$$
\overline{\overline{\mathbf{M}}} \ddot{\mathbf{X}}^{t}=\mathbf{F}^{t}
$$

Let the body-links be defined by nonlinear equations of the following type:

$$
f_{\mathrm{i}}\left(\mathbf{X}^{t}\right)=f_{\mathrm{i}}\left(X_{1}^{t}, X_{2}^{t}, \ldots, X_{\mathrm{n}}^{t}\right)=b_{\mathrm{i}},
$$

where $\mathbf{X}^{t}$ is the vector representing the position of every body of the system, and $b_{\mathrm{i}}$ is a constant value. The body-links will be included into the system dynamics via Lagrange multipliers. In order to include the constraint equations (body-links) within the iterative body dynamics solver, the following numerical linearization is used:

$$
\begin{gathered}
f_{\mathrm{i}}\left(X_{1}^{t, k+1}, X_{2}^{t, k+1}, \ldots, X_{\mathrm{n}}^{t, k+1}\right)=f_{\mathrm{i}}\left(X_{1}^{t, k}, X_{2}^{t, k}, \ldots, X_{\mathrm{n}}^{t, k}\right)+\sum_{\mathrm{j}}\left(\frac{\partial f_{\mathrm{i}}}{\partial x_{\mathrm{j}}}\right)^{t, k}\left(X_{\mathrm{j}}^{t, k+1}-X_{\mathrm{j}}^{t, k}\right) \\
=b_{\mathrm{i}},
\end{gathered}
$$

in vector form:

$$
\left(\nabla f_{\mathrm{i}}\right)^{t, k} \cdot \mathbf{X}^{t, k+1}=b_{\mathrm{i}}-f_{\mathrm{i}}\left(\mathbf{X}^{t, k}\right)+\left(\nabla f_{\mathrm{i}}\right)^{t, k} \cdot \mathbf{X}^{t, k},
$$

where $k$ is the $\mathrm{k}$-th iteration of the iterative scheme used to solve the dynamic system. The previous linearization ensures that as $\left\|\mathbf{X}^{t, k}-\mathbf{X}^{t, k+1}\right\| \rightarrow 0 \Rightarrow f_{\mathrm{i}}\left(\mathbf{X}^{t, k+1}\right) \rightarrow b_{\mathrm{i}}$ and the body-link is fulfilled.

We use an alpha Bossak-Newmark scheme as a temporal integrator scheme for the dynamic equations. In such scheme, displacements $x_{j}^{t+\Delta t, k+1}$ depend on accelerations $\ddot{x}_{j}^{t+\Delta t, k+1}$ as follows:

$$
\mathbf{X}^{t, k+1}=\mathbf{X}^{t-\Delta t}+\Delta \mathrm{t} \dot{\mathbf{X}}^{t-\Delta t}+\frac{\Delta \mathrm{t}^{2}}{2}\left((1-2 \beta) \ddot{\mathbf{X}}^{t-\Delta t}+2 \beta \ddot{\mathbf{X}}^{t, k+1}\right) \text {. }
$$

Introducing the Newmark scheme into the linearized body-link:

$$
\begin{gathered}
\left(\nabla f_{i}\right)^{t, k} \cdot\left(\mathbf{X}^{t-\Delta t}+\Delta t \dot{\mathbf{X}}^{t-\Delta t}+\Delta t^{2}\left((0.5-\beta) \ddot{\mathbf{X}}^{t, k+1}+\beta \ddot{\mathbf{X}}^{t-\Delta t}\right)\right) \\
=b_{i}-f_{i}\left(\mathbf{X}^{t, k}\right)+\left(\nabla f_{i}\right)^{t, k} \cdot \mathbf{X}^{t, k} .
\end{gathered}
$$


The previous equation can be written as:

$$
\begin{aligned}
\left(\nabla f_{i}\right)^{t, k} \cdot \ddot{\mathbf{X}}^{t, k+1}= & \frac{1}{\Delta t^{2} \beta}\left(b_{i}-f_{i}\left(\mathbf{X}^{t, k}\right)+\left(\nabla f_{i}\right)^{t, k} \cdot \mathbf{X}^{t, k}\right)- \\
& -\left(\nabla f_{i}\right)^{t, k} \cdot\left(\frac{\mathbf{X}^{t-\Delta t}}{\Delta t^{2} \beta}+\frac{\dot{\mathbf{X}}^{t-\Delta t}}{\Delta t \beta}+\frac{(0.5-\beta)}{\beta} \ddot{\mathbf{X}}^{t-\Delta t}\right) .
\end{aligned}
$$

After reordering terms:

$$
\left(\nabla f_{i}\right)^{t, k} \cdot \ddot{\mathbf{X}}^{t, k+1}=\frac{1}{\Delta t^{2} \beta}\left(b_{i}-f_{i}\left(\mathbf{X}^{t, k}\right)\right)+\left(\nabla f_{i}\right)^{t, k} \cdot \ddot{\mathbf{X}}^{t, k}
$$

The set of linearized body-links can be written as:

$$
\sum_{j} \mathrm{a}_{i j}^{t, k} \ddot{X}_{j}^{t, k+1}=c_{i}^{t, k}
$$

where $\overline{\overline{\mathbf{A}}}^{t, k}=\left[\mathrm{a}_{i j}^{t, k}\right]$ is the Jacobian matrix $\mathrm{a}_{i j}^{t, k}=\left(\frac{\partial f_{i}}{\partial x_{j}}\right)^{t, k}$ and $\boldsymbol{C}^{t, k}=\left[c_{i}^{t, k}\right]$ :

$$
\begin{aligned}
c_{i}^{t, k}=\frac{1}{\Delta t^{2} \beta}\left[b_{i}\right. & \left.-f_{i}\left(\mathbf{X}^{t, k}\right)+\sum_{j} \mathrm{a}_{i j}^{t, k} X_{j}^{t, k}\right] \\
& -\sum_{j} \mathrm{a}_{i j}^{t, k}\left(\frac{X_{j}^{t-\Delta t}}{\Delta t^{2} \beta}+\frac{\dot{X}_{j}^{t-\Delta t}}{\Delta t \beta}+\frac{(0.5-\beta)}{\beta} \ddot{X}_{j}^{t-\Delta t}\right),
\end{aligned}
$$

or

$$
c_{i}^{t, k}=\frac{1}{\Delta t^{2} \beta}\left[b_{i}-f_{i}\left(\mathbf{X}^{t, k}\right)\right]+\sum_{j} a_{i j}^{t, k} \ddot{X}_{j}^{t, k} .
$$

Then, the imposition of nonlinear body-links via Lagrange multipliers is carried out as follows:

$$
\left[\begin{array}{cc}
\overline{\overline{\mathbf{M}}} & \left(\overline{\overline{\mathbf{A}}}^{T}\right)^{t, k} \\
\overline{\overline{\mathbf{A}}}^{t, k} & 0
\end{array}\right]\left[\begin{array}{l}
\ddot{\mathbf{X}}^{t, k+1} \\
\lambda^{t, k+1}
\end{array}\right]=\left[\begin{array}{l}
\mathbf{F}^{t, k} \\
\boldsymbol{C}^{t, k}
\end{array}\right] .
$$

Finally, reaction forces are obtained from:

$$
\mathbf{R}^{t, k}=-\left(\overline{\bar{A}}^{T}\right)^{t, k} \lambda^{t, k}
$$

\subsubsection{Body-links with Alpha Bossak-Newmark integration}

The alpha Bossak-Newmark scheme solves the following equation to obtain the body acceleration at time $\mathrm{t}$ :

$$
(1-\alpha) \overline{\overline{\mathbf{M}}} \ddot{\mathbf{X}}^{t}+\alpha \overline{\overline{\mathbf{M}}} \ddot{\mathbf{X}}^{t-\Delta t}=\mathbf{F}^{t}+\mathbf{R}^{t},
$$

where $\mathbf{R}^{t}=-\overline{\overline{\mathbf{A}}}^{T} \lambda^{t}$ are the reaction forces due to body-links. Then:

$$
\overline{\overline{\mathbf{M}}}(1-\alpha) \ddot{\mathbf{X}}^{t}+\overline{\overline{\mathbf{A}}}^{T} \lambda^{t}=\mathbf{F}^{t}-\alpha \mathbf{M} \ddot{\mathbf{X}}^{t-\Delta t} \text {. }
$$

Introducing the body-link constraints into the multibody dynamics:

$$
\left[\begin{array}{cc}
\overline{\overline{\mathbf{M}}} & \overline{\overline{\mathbf{A}}}^{\boldsymbol{T}} \\
\overline{\overline{\mathbf{A}}} & \mathbf{0}
\end{array}\right]\left[\begin{array}{c}
(1-\alpha) \ddot{\mathbf{X}}^{t} \\
\lambda^{t}
\end{array}\right]=\left[\begin{array}{c}
\mathbf{F}^{t}-\alpha \overline{\overline{\mathbf{M}}} \ddot{\mathbf{X}}^{t-\Delta t} \\
(1-\alpha) \boldsymbol{C}^{t}
\end{array}\right] .
$$




\subsection{Body dynamics iterative solver}

The dynamic solver is implemented based on three nested loops. A relaxation algorithm based on the Aitken's method [42] is used to speed up convergence within each loop. Figure 7 shows the algorithm of the numerical scheme for the dynamics solver. A description of each lop is given next.

\subsubsection{Time marching loop}

The time marching loop is the outer loop, and each iteration corresponds to a time step. Information from the previous time step is used as initial guests for the current step. The Aitken's method is used to relax body velocities with the aim of speeding up convergence.

\subsubsection{Solver loop}

The solver loop is where the wave diffraction-radiation problem and hydrodynamic loads are solved. In each iteration, the linear system corresponding to the wave diffraction-radiation problem must be solved. Iterations are carried out until convergence of hydrodynamic loads along with body kinematics is reached. The Aitken's method is used to relax body accelerations with the aim of speeding up convergence.

\subsubsection{Body dynamics loop}

The body dynamics loop is the inner loop. Within this loop, wave diffraction and radiation loads remain unchanged, hydrostatic forces are updated based on body position, and the rest of external loads will be updated in each iteration. The Aitken's method [42] is used to relax body displacements with the aim of speeding up convergence. 


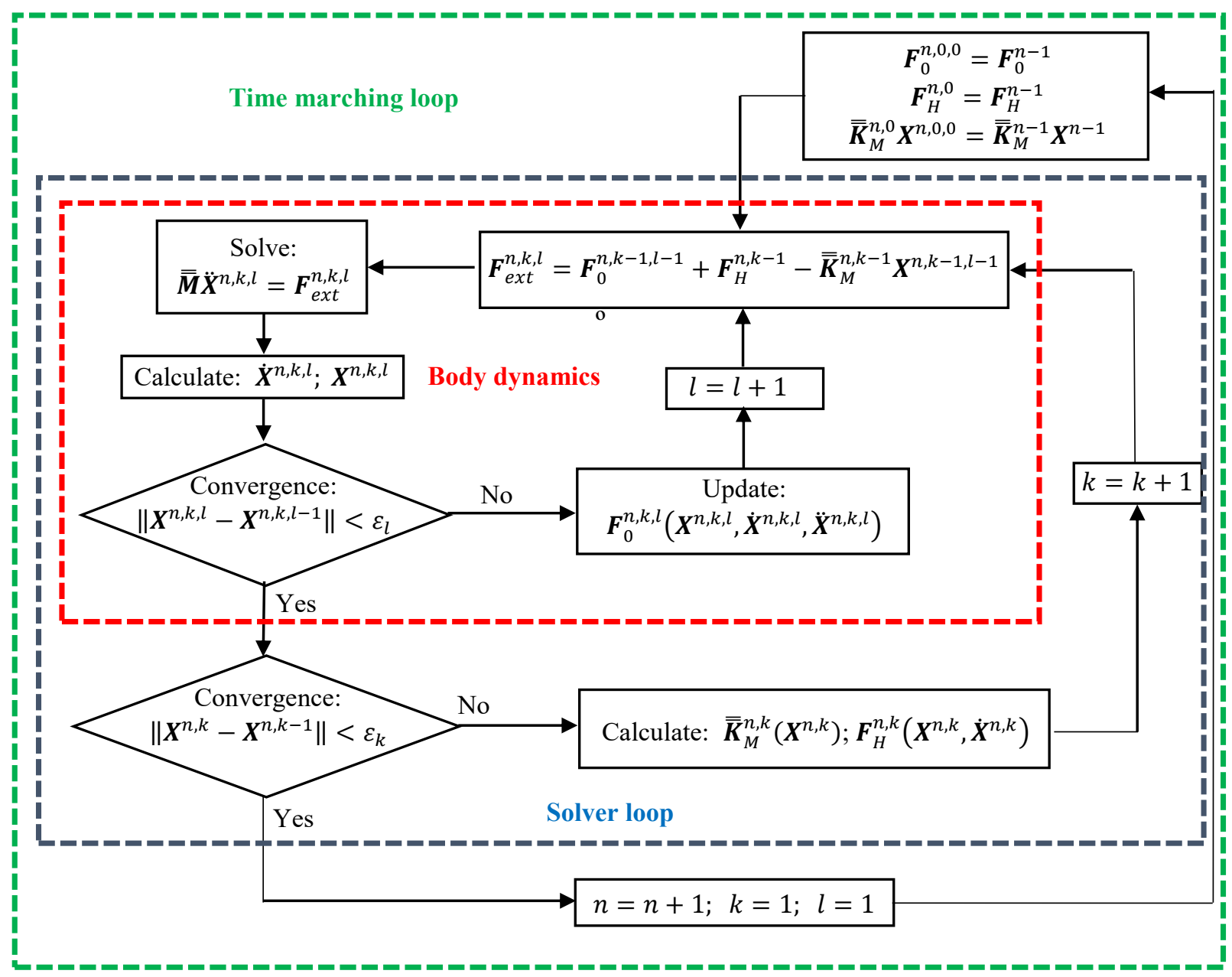

Figure 7: Body dynamics solver algorithm integrated with the wave diffraction-radiation solver and a mooring solver. 
PART II: VERIFICATION AND

VALIDATION 


\section{Chapter 5. SEAKEEPING: VERIFICATION AND VALIDATION}

\subsection{Introduction}

This chapter aims at the verification of the numerical schemes and the validation of the mathematical models introduced in the previous chapters for seakeeping problems. By verification we mean the appropriateness and accuracy of the numerical schemes to solve the corresponding mathematical model. By validation we mean the appropriateness of the mathematical-numerical models to reproduce the underlying physics. Hence, verification will be carry out by comparing numerical solution against analytical solution, while validation will be carried out comparing against experimental results. Moreover, inter-code comparisons against other numerical schemes will be carried out for cases where neither analytical solutions nor experimental data are available.

\subsection{Verification case: Waves refracted by a vertical circular cylinder}

In order to verify some of the proposed numerical schemes, the problem of a monochromatic wave interacting with a fix bottom mounted circular cylinder will be treated. This case study has been chosen for having an analytical solution, and it was found by McCamy and Fuchs in 1954 [43]. The velocity potential for a monochromatic wave travelling along the $\mathrm{OX}$ axis in cylindrical coordinates is given by:

$$
\phi^{I}=\operatorname{Re}\left\{\frac{i A g}{\omega} \frac{\cosh (k(H+z))}{\cosh (k H)}\left[\sum_{m \geq 0} \epsilon_{m} J_{m}(k r) \cos (m \theta)\right] \exp (i \omega t)\right\},
$$

where $(r, \theta, z)$ are the cylindrical coordinates; $A$ is the wave amplitude; $\omega$ is the wave frequency; $H$ is the water depth; $J_{m}$ are the Bessel functions of the first kind; and $\epsilon_{0}=1$, and $\epsilon_{m}=2(-i)^{m}$ if $m>0$. The velocity potential of the wave diffracted by the cylinder is given by:

$$
\phi^{S}=R e\left\{\frac{i A g}{\omega} \frac{\cosh (k(H+z))}{\cosh (k H)}\left[\sum_{m \geq 0}-\epsilon_{m} \frac{J_{m}^{\prime}(k R)}{H_{m}^{(2)^{\prime}}(k R)} H_{m}^{(2)}(k r) \cos (m \theta)\right] \exp (i \omega t)\right\},
$$

where $\left({ }^{\prime}\right)$ denotes derivatives respect to the argument $(k R) ; R$ is the radius of the cylinder; and $H_{m}^{(2)}$ are the Hankel function of the second kind. Summing up Eqs. (5-1)-(5-2), we obtain the analytical solution for the waves diffracted by vertical circular cylinder problem. 


$$
\begin{aligned}
\phi=R e\left\{\frac{i A g}{\omega}\right. & \frac{\cosh (k(H+z))}{\cosh (k H)}\left[\sum _ { m \geq 0 } \epsilon _ { m } \left(J_{m}(k r)\right.\right. \\
& \left.\left.\left.-\frac{J_{m}^{\prime}(k R)}{H_{m}^{(2)^{\prime}}(k R)} H_{m}^{(2)}(k r)\right) \cos (m \theta)\right] \exp (i \omega t)\right\} .
\end{aligned}
$$

The dynamic pressure $P^{\phi}=-\rho \partial_{t} \phi$, is given by:

$$
\begin{aligned}
P^{\phi}=R e\{A \rho g & \frac{\cosh (k(H+z))}{\cosh (k H)}\left[\sum _ { m \geq 0 } \epsilon _ { m } \left(J_{m}(k r)\right.\right. \\
& \left.\left.\left.-\frac{J_{m}^{\prime}(k R)}{H_{m}^{(2)^{\prime}}(k R)} H_{m}^{(2)}(k r)\right) \cos (m \theta)\right] \exp (i \omega t)\right\} .
\end{aligned}
$$

The horizontal force induced over the cylinder is obtained by integration of $P^{\phi}$ over the cylinder surface. This exact solution is given by the following expression:

$$
F_{x}=\operatorname{Re}\left\{2 i A R \rho g \pi \frac{\tanh (k H)}{k}\left(J_{1}(k R)-\frac{J_{1}^{\prime}(k R)}{H_{1}^{(2)^{\prime}}(k R)} H_{m}^{(2)}(k R)\right) \exp (i \omega t)\right\},
$$

And will be compared next against numerical results obtained by the analytical solution against numerical results based on the schemes proposed in this thesis, for the specific case of $R=1 \mathrm{~m}, H=$ $1 \mathrm{~m}, A=0.1 \mathrm{~m}, L=2 \mathrm{~m}$. Using $\rho=1025 \mathrm{~kg} / \mathrm{m}^{3}, \mathrm{~g}=9.81 \mathrm{~m} / \mathrm{s}^{2}$. Using the dispersion relation for first-order waves, the corresponding wave frequency and period are $\omega=5.5411 \mathrm{rad} / \mathrm{s}$, and $T=$ $1.1319 s$.

The simulation is carried out using a circular domain with a radius of 6 meters. The mesh size is set to 0.1 meters in an inner volume within a radius of 2 meters. In the outer volume, the mesh size grows smoothly up to 0.5 meters. The mesh created consists of 35914 nodes and 188715 tetrahedral elements.

Figure 8 compares the contour lines of the free surface elevation, Figure 9 compares the dynamic pressure distribution, and Figure 10 compares the horizontal force induced over the cylinder at times $\mathrm{t}=\mathrm{nT}$. Notice that the FEM solution is able to reproduce the analytical one with high accuracy. 


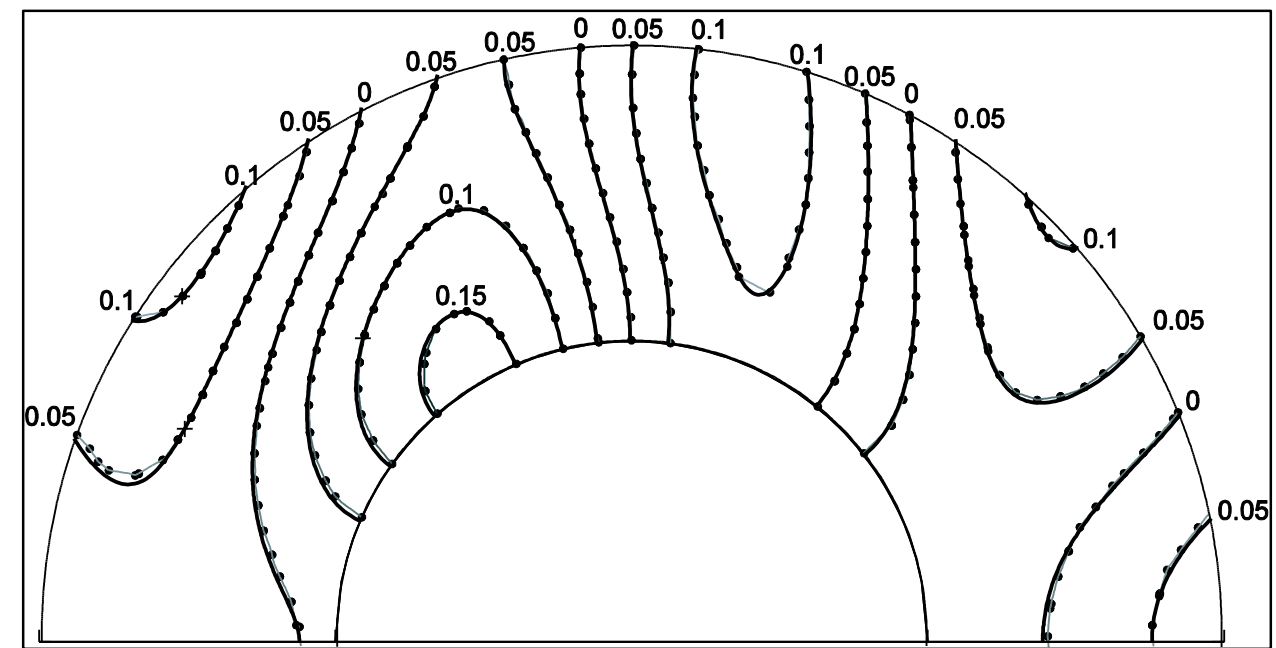

Figure 8: Waves refracted by a vertical circular cylinder: comparison of free surface elevation obtained by the analytical solution [43] and the FEM solution.

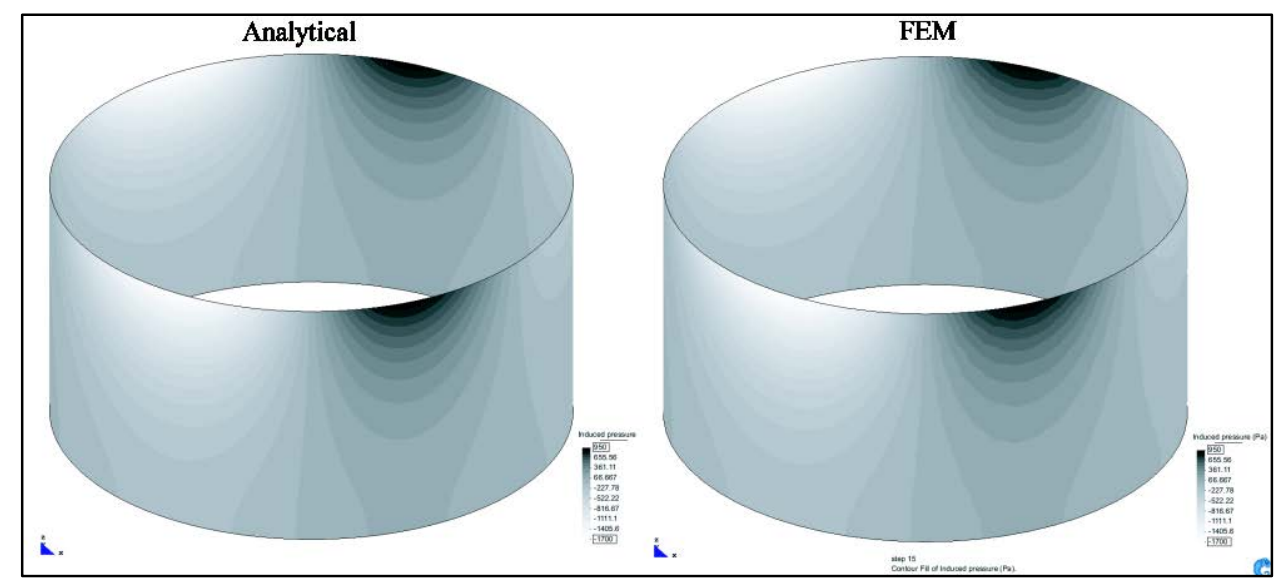

Figure 9: Waves refracted by a vertical circular cylinder: comparison of pressure distribution over the cylinder obtained by the analytical solution [43] and the FEM solution. Both pressure distributions are shown using the same color scale, with a maximum value of $950 \mathrm{~Pa}$ and a minimum of $-1700 \mathrm{~Pa}$.

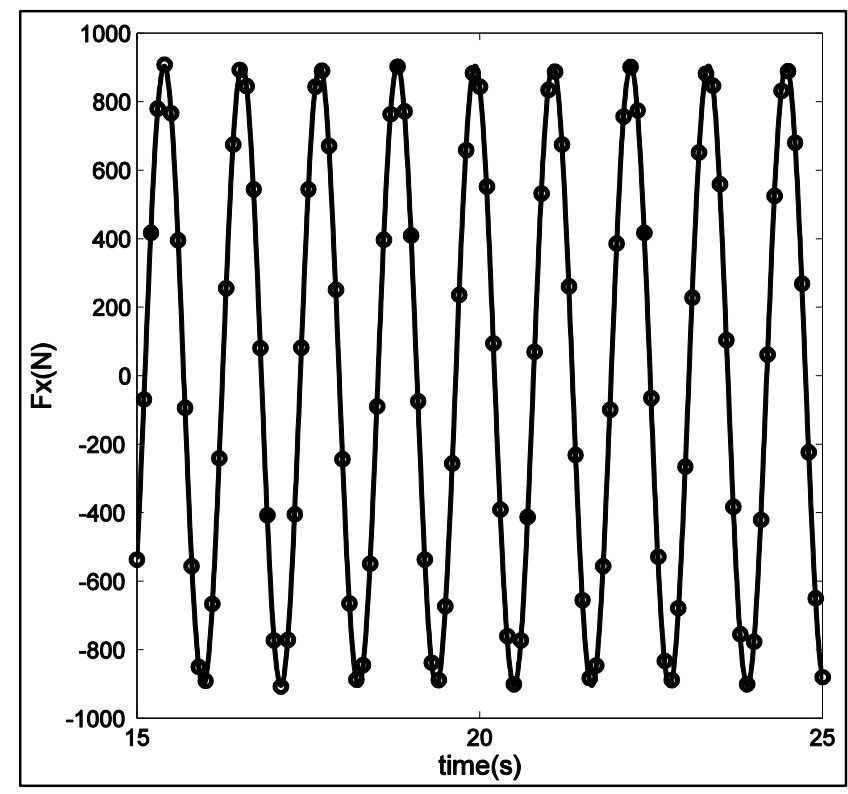

Figure 10: Waves refracted by a vertical circular cylinder: comparison of force induced over the cylinder obtained by the analytical solution [43] and FEM. 


\subsection{Verification case: Standing wave dissipation by pressure}

\subsubsection{First-order analytical solution for pressure based wave dissipation}

The first-order wave equations with a free surface pressure such that $P=\tau \partial_{z} \varphi$ are:

$$
\begin{array}{ll}
\nabla^{2} \varphi=0 & \text { in } \Omega, \\
\partial_{t t} \varphi+\frac{\tau}{\rho} \partial_{z t} \varphi+g \partial_{z} \varphi=0 & \text { on } z=0, \\
\partial_{z} \varphi=0 & \text { on } z=-H, \\
\zeta=-\frac{1}{g} \partial_{t} \varphi-\frac{\tau}{\rho g} \partial_{z} \varphi & \text { on } z=0,
\end{array}
$$

where $\varphi$ is the velocity potential, $\zeta$ is the free surface elevation.

When $\tau^{2} \leq \frac{4 g \rho^{2}}{\left|\boldsymbol{k}_{i}\right| \tanh \left(\left|\boldsymbol{k}_{i}\right| H\right)}$ there exists an analytical solution to the above stated problem, which is:

$$
\varphi=\sum_{i} \exp \left(-\frac{1}{2} B_{i} t\right) A_{i} \frac{g}{\omega_{i}} \frac{\cosh \left(\left|\boldsymbol{k}_{i}\right|(H+z)\right)}{\cosh \left(\left|\boldsymbol{k}_{i}\right| H\right)} \cos \left(\left|\boldsymbol{k}_{i}\right|\left(x \cos \beta_{i}+y \sin \beta_{i}-\omega_{i} t+\alpha_{i}\right)\right. \text {, }
$$

where $B_{i}=\frac{\tau}{\rho}\left|\boldsymbol{k}_{i}\right| \tanh \left(\left|\boldsymbol{k}_{i}\right| H\right)$ and $\omega_{i}^{2}=g\left|\boldsymbol{k}_{i}\right| \tanh \left(\left|\boldsymbol{k}_{i}\right| H\right)-\frac{B_{i}^{2}}{4}>0$. The dispersion relation can be re-written as:

$$
\omega_{i}^{2}=g\left|\boldsymbol{k}_{i}\right| \tanh \left(\left|\boldsymbol{k}_{i}\right| H\right)-\left[\frac{\tau}{\rho}\left|\boldsymbol{k}_{i}\right| \tanh \left(\left|\boldsymbol{k}_{i}\right| H\right)\right]^{2} / 4,
$$

and the analytical solution for the free surface elevation is:

$$
\begin{aligned}
\zeta= & \sum_{i}-\exp \left(-\frac{1}{2} B_{i} t\right) A_{i} \sin \left(\left|\boldsymbol{k}_{i}\right|\left(x \cos \beta_{i}+y \sin \beta_{i}\right)-\omega_{i} t+\alpha_{i}\right) \\
& +\sum_{i} \frac{1}{2} B_{i} \exp \left(-\frac{1}{2} B_{i} t\right) A_{i} \frac{1}{\omega_{i}} \cos \left(\left|\boldsymbol{k}_{i}\right|\left(x \cos \beta_{i}+y \sin \beta_{i}\right)-\omega_{i} t+\alpha_{i}\right) \\
& -\sum_{i} \frac{\tau}{\rho} \exp \left(-\frac{1}{2} B_{i} t\right) A_{i} \frac{\left|\boldsymbol{k}_{i}\right|}{\omega_{i}} \tanh \left(\left|\boldsymbol{k}_{i}\right| H\right) \cos \left(\left|\boldsymbol{k}_{i}\right|\left(x \cos \beta_{i}+y \sin \beta_{i}\right)-\omega_{i} t+\alpha_{i}\right)
\end{aligned}
$$

\subsubsection{Standing wave dissipation}

Let's consider a finite domain with ten meters in length, two meters in depth, and half a meter in width. We will consider the problem of dissipating a standing wave along the longitudinal direction by applying a pressure on the free surface proportional to the vertical wave velocity. From Eq. (5-7), the analytical solution for the velocity potential of this problem is:

$$
\varphi=\exp \left(-B^{*} \tau t\right) \frac{g}{\omega} \frac{\cosh (\mathrm{k}(\mathrm{H}+z))}{\cosh (\mathrm{kH})}(\cos (\mathrm{k} x-\omega t)+\cos (\mathrm{k} x+\omega t)),
$$


where $B^{*}=\frac{1}{2} \mathrm{k} \tanh (\mathrm{kH}) / \rho, \rho=1025 \mathrm{~kg} / \mathrm{m}^{3}$, and $\tau$ is the damping coefficient such that $P(\boldsymbol{x}, t)=$ $\tau \partial_{z} \varphi(x, t)$. The wave frequency $\omega$ is obtained from Eq. (5-8). The free surface elevation can be obtained from Eq.(5-9):

$$
\begin{aligned}
\zeta=-\exp \left(-B^{*} \tau t\right) & (\sin (k x-\omega t)-\sin (k x+\omega t)) \\
& +\tau\left(\frac{B^{*}}{g}-\frac{k}{\rho \omega} \tanh (k H)\right) \exp \left(-B^{*} \tau t\right)(\cos (k x-\omega t) \\
& +\cos (k x+\omega t)) .
\end{aligned}
$$

It is defined the critical value $\tau_{c}$ as the one that yields to $\omega=0$ :

$$
\tau_{c}=\frac{2 \rho g}{\sqrt{\mathrm{gk} \tanh (\mathrm{k} H)}},
$$

where the analytical solution exist for $\tau<\tau_{c}$. Table 3 provides the wave properties used for this verification. The wave length was chosen to be the length of the tank, while three different values of $\tau / \tau_{c}$ were considered.

Figure 11 shows the evolution of the free surface for the $\tau / \tau_{c}=0.9$ case, where the wave amplitude is mostly damped out in less than one second. Figure 12 shows the time evolution of the free surface elevation of the central point for the three cases under analysis. As expected, as $\tau / \tau_{c}$ gets closer to unity, the faster the wave dissipation occurs. A very good fitting between the numerical and analytical solution is observed.

Table 3: Standing wave particulars.

\begin{tabular}{|c|c|c|c|}
\hline & $\boldsymbol{\tau} / \boldsymbol{\tau}_{\boldsymbol{c}}=\mathbf{0 . 1}$ & $\boldsymbol{\tau} / \boldsymbol{\tau}_{\boldsymbol{c}}=\mathbf{0 . 5}$ & $\boldsymbol{\tau} / \boldsymbol{\tau}_{\boldsymbol{c}}=\mathbf{0 . 9}$ \\
\hline $\boldsymbol{\omega}(\mathbf{r a d} / \mathbf{s})$ & 2.2773 & 1.982 & 0.9976 \\
\hline $\boldsymbol{T}=\mathbf{2 \pi} / \boldsymbol{\omega}(\mathbf{s})$ & 2.7591 & 3.17 & 6.298 \\
\hline $\boldsymbol{B}^{*}$ & 0.000261 & 0.000261 & 0.000261 \\
\hline $\boldsymbol{\tau}\left(\mathbf{N s} / \mathbf{m}^{\mathbf{3}}\right)$ & 878.4 & 4392 & 7905 \\
\hline
\end{tabular}

\subsection{Validation case: Seakeeping of a GVA 4000 Semisubmersible platform}

A comparison between experimental data [44] and the numerical models introduced in section 2.2 of this thesis is carried out by studying the seakeeping behavior of the GVA 4000 semisubmersible platform. The results to be compared are the heave and pitch RAOs in heading seas, for a range of wave periods between 6 and 32 seconds.

The platform displacement is 25940 tons. The center of gravity is located $21.35 \mathrm{~m}$ above the keel, and the horizontal position corresponds to the geometric center of the platform. The radii of inertia are $r_{x x}=30.40 \mathrm{~m}, r_{y y}=31.06 \mathrm{~m}$, and $r_{z z}=37.54 \mathrm{~m}$. The geometry of the GVA 4000 can be found in [44]. 


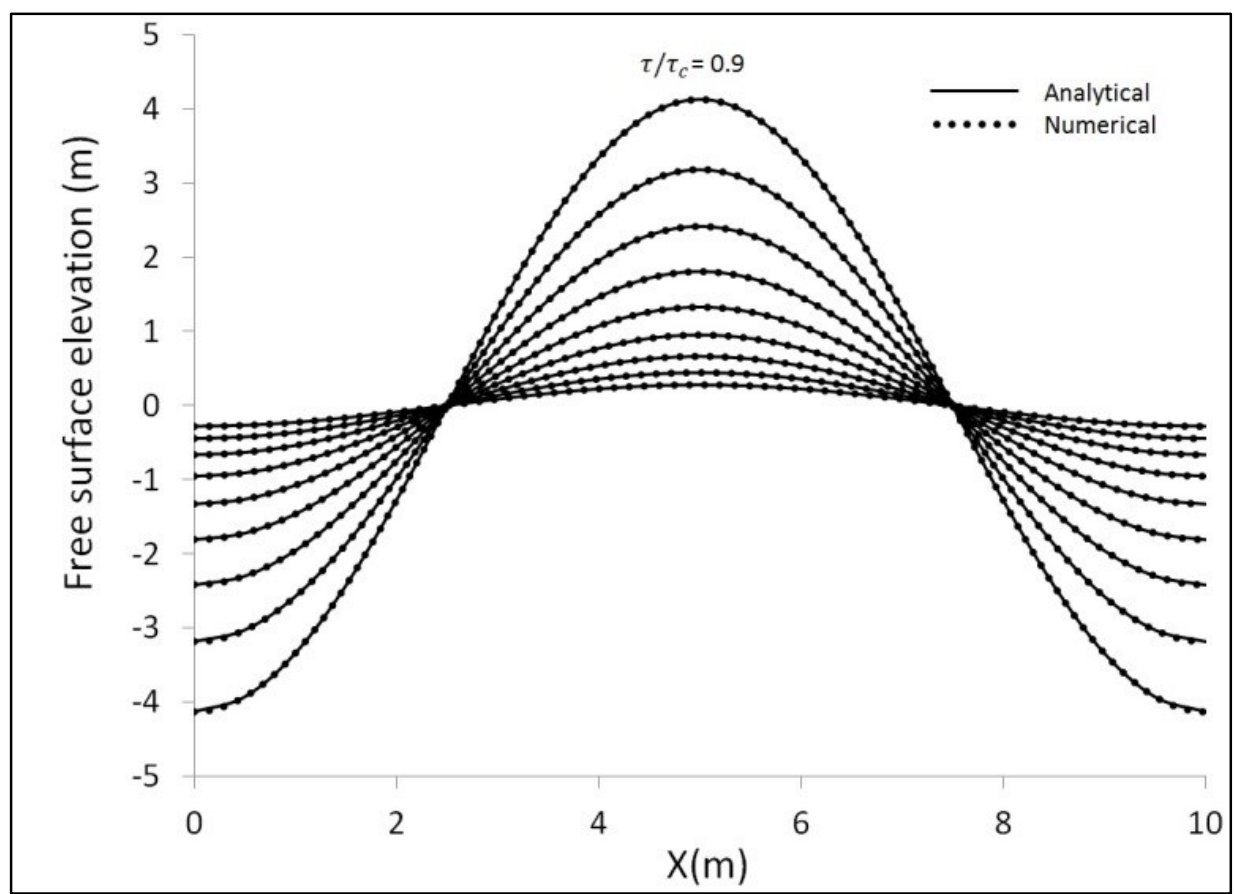

Figure 11: Standing wave dissipation: snapshots of free surface evolution from time $=0 \mathrm{~s}$ to time $=0.8 \mathrm{~s}$ in intervals of 0.1 seconds. Analytical solution (solid line) versus numerical solution (dots).

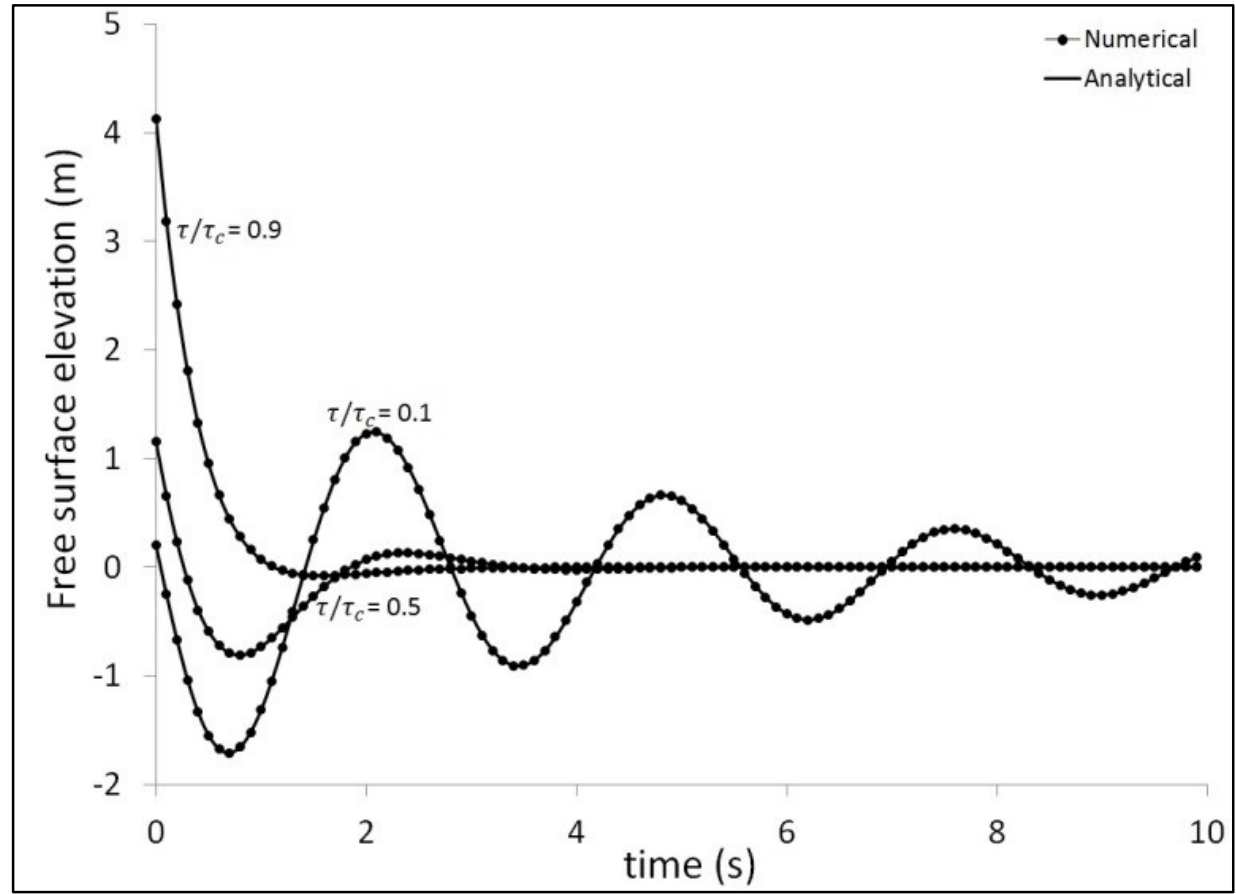

Figure 12: Standing wave dissipation: time evolution of central point of the free surface for $\tau / \tau_{\mathrm{c}}=0.1, \tau / \tau_{\mathrm{c}}=0.5$, and $\tau / \tau_{\mathrm{c}}=0.9$. Analytical solution (solid line) versus numerical solution (dots).

Based on [44], the model tests were carried out with the surge movement constraint by the action of a pre-stressed spring whose mission is to keep in place the structure during testing. This spring creates also a pitching moment, and therefore, the pitch movement will be affected.

Since no data regarding the pre-stressed spring used by the model basin was available, it could not be included in the simulation. Instead, simulations have been carried out in two cases: no surge and free surge. Figure 13 compares the experimental and numerical results from this thesis. It is observed 
that experimental results lay within the range of the two cases contemplated in the numerical simulations.

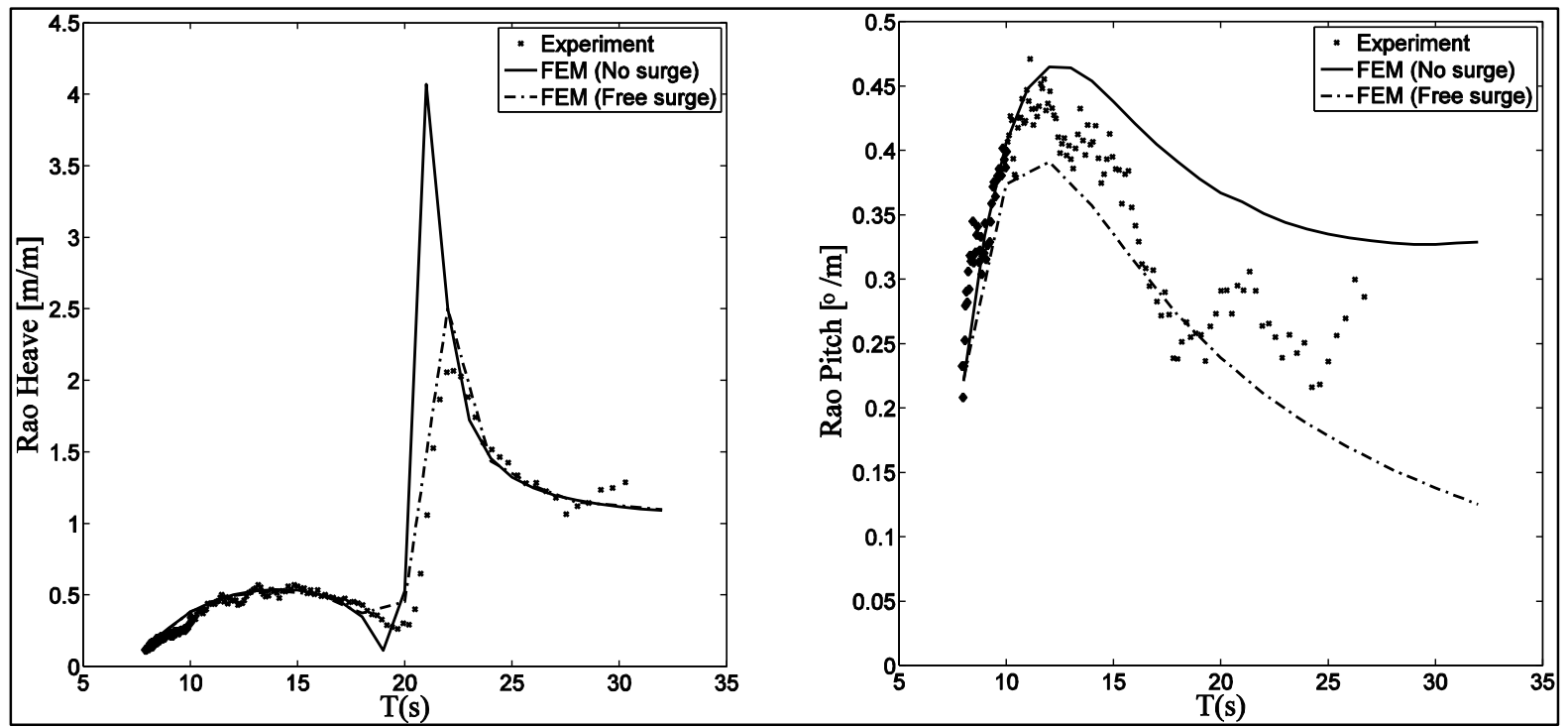

Figure 13: GVA 4000 RAOs comparison between experiments and present work.

\subsection{Inter-code comparison: Freely floating tension leg platform}

In this section we analyze the seakeeping behavior of a freely floating tension leg platform (TLP). The platform used is the ISSC TLP [45]. The platform mass is obtained by matching the displacement of the platform. The gravity center position and radii of inertia are provided in Table 4 . Figure 14 shows the mesh used for the present case study.

The gravity value used is $g=9.80665 \mathrm{~m} / \mathrm{s}^{2}$, density $\rho=1025 \mathrm{~kg} / \mathrm{m}^{3}$, and water depth is assumed to be infinite. Simulations were carried out for periods ranging between eight and fifteen seconds for three different wave headings. Figure 15 compares the RAOs obtained by the present FEM model and RAOs obtained by the well-known program WAMIT [46], which is based on the BEM and solves in the frequency domain. Results from WAMIT were obtained for the example case described in [46]. Results show good agreement between both numerical approaches.

Table 4: ISSC TLP particulars

\begin{tabular}{|c|c|}
\hline XG (m) & 0 \\
\hline YG (m) & 0 \\
\hline ZG (m) & 3 \\
\hline Ixx/Mass $\left.\mathbf{( m}^{\mathbf{2}}\right)$ & 38.876 \\
\hline Iyy/Mass $\left.\mathbf{( m}^{\mathbf{2}}\right)$ & 38.876 \\
\hline Izz/Mass $\left.\mathbf{( m}^{\mathbf{2}}\right)$ & 42.420 \\
\hline
\end{tabular}




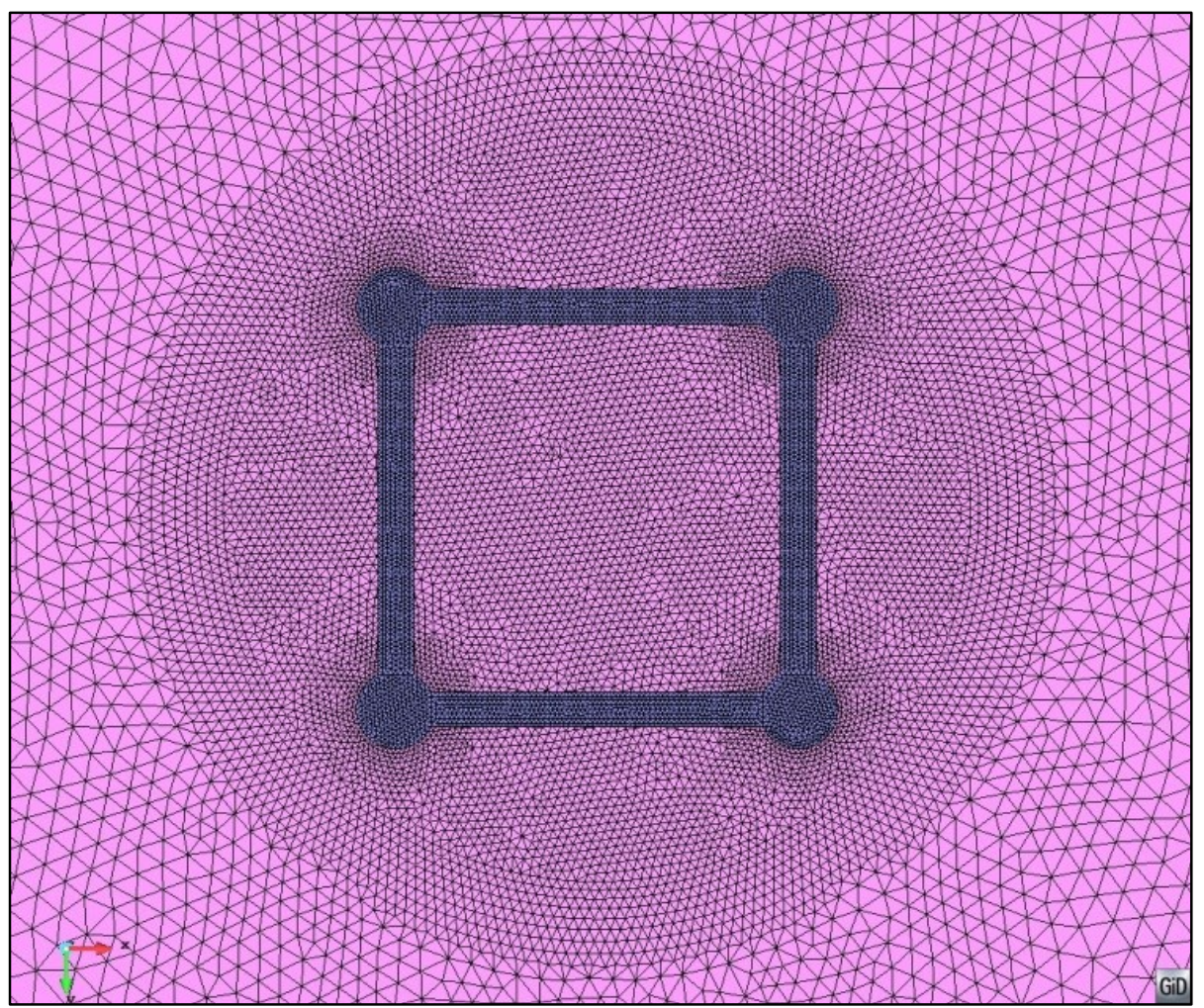

Figure 14: FEM mesh for ISSC TLP platform and free surface.

\subsection{Mean drift forces on hemisphere}

This is the case of estimating the mean drift forces of a hemisphere. The analytical solution for the fix hemisphere was obtained Fernandes and Levy [47], and for the freely floating hemisphere was obtained by Kudou [48] and reported by Pinkster [49]. In this section, we compared against analytical results for a hemisphere with particulars given in Table 5. Figure 16 (left) shows the mesh used for the calculations. It can be observed that mesh refinement is required in the area of the waterline in order to obtain accurate results of mean drift forces. Figure 16 (right) shows a snapshot of the wave elevation around the hemisphere. Figure 17 compares the analytical results against the numerical results. A good agreement is observed for the whole range of waves analyzed.

Table 5: hemisphere particulars

\begin{tabular}{|c|c|}
\hline Depth & Infinite \\
\hline Mass & Displacement \\
\hline Radius & $1 \mathrm{~m}$ \\
\hline CG & $(0,0,0)$ \\
\hline Number of tetrahedrons & 274283 \\
\hline Number of triangles & 22082 \\
\hline
\end{tabular}



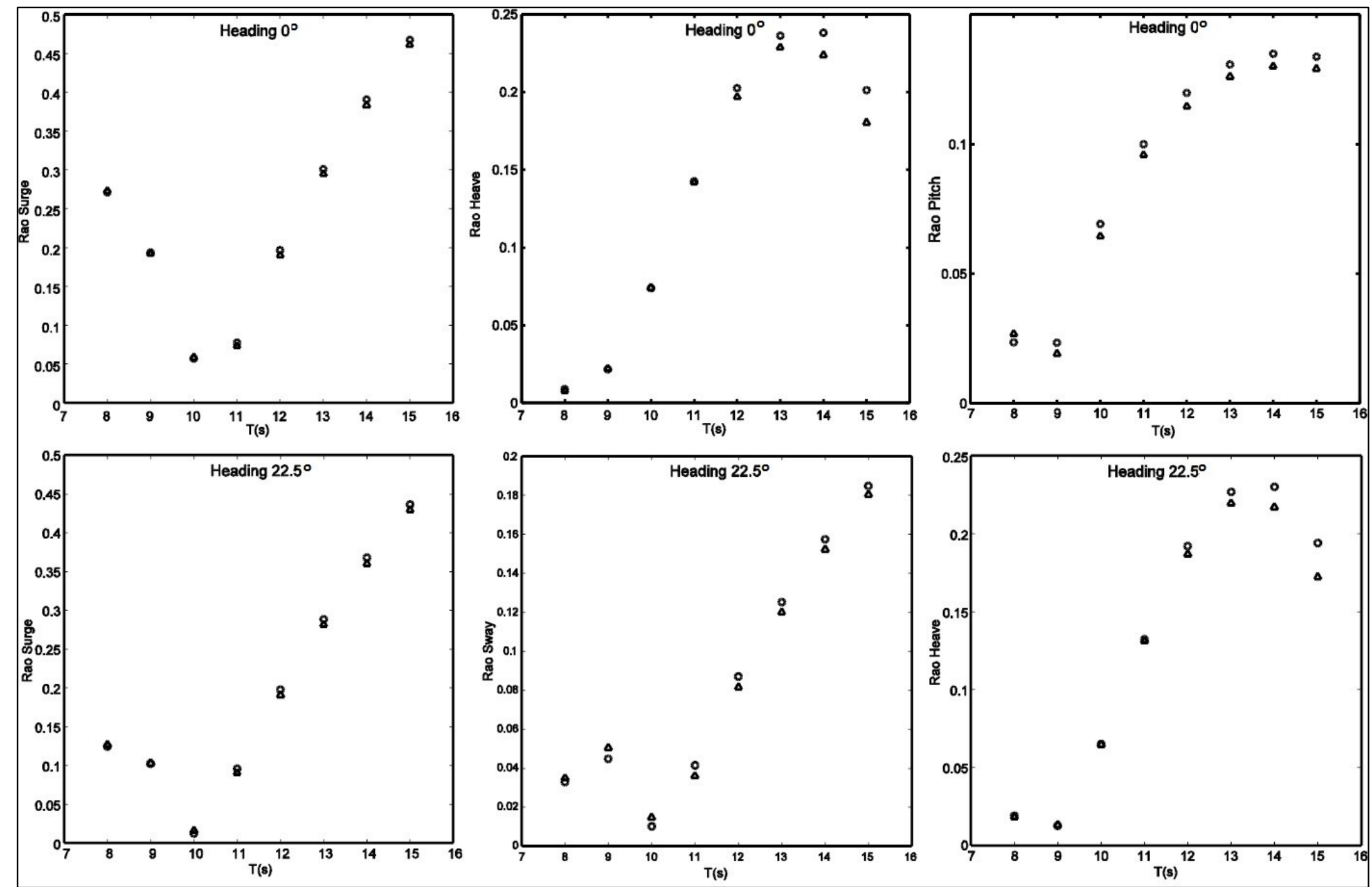

Figure 15: Response amplitude operator for freely floating TLP. Circles: WAMIT; triangles: FEM. 


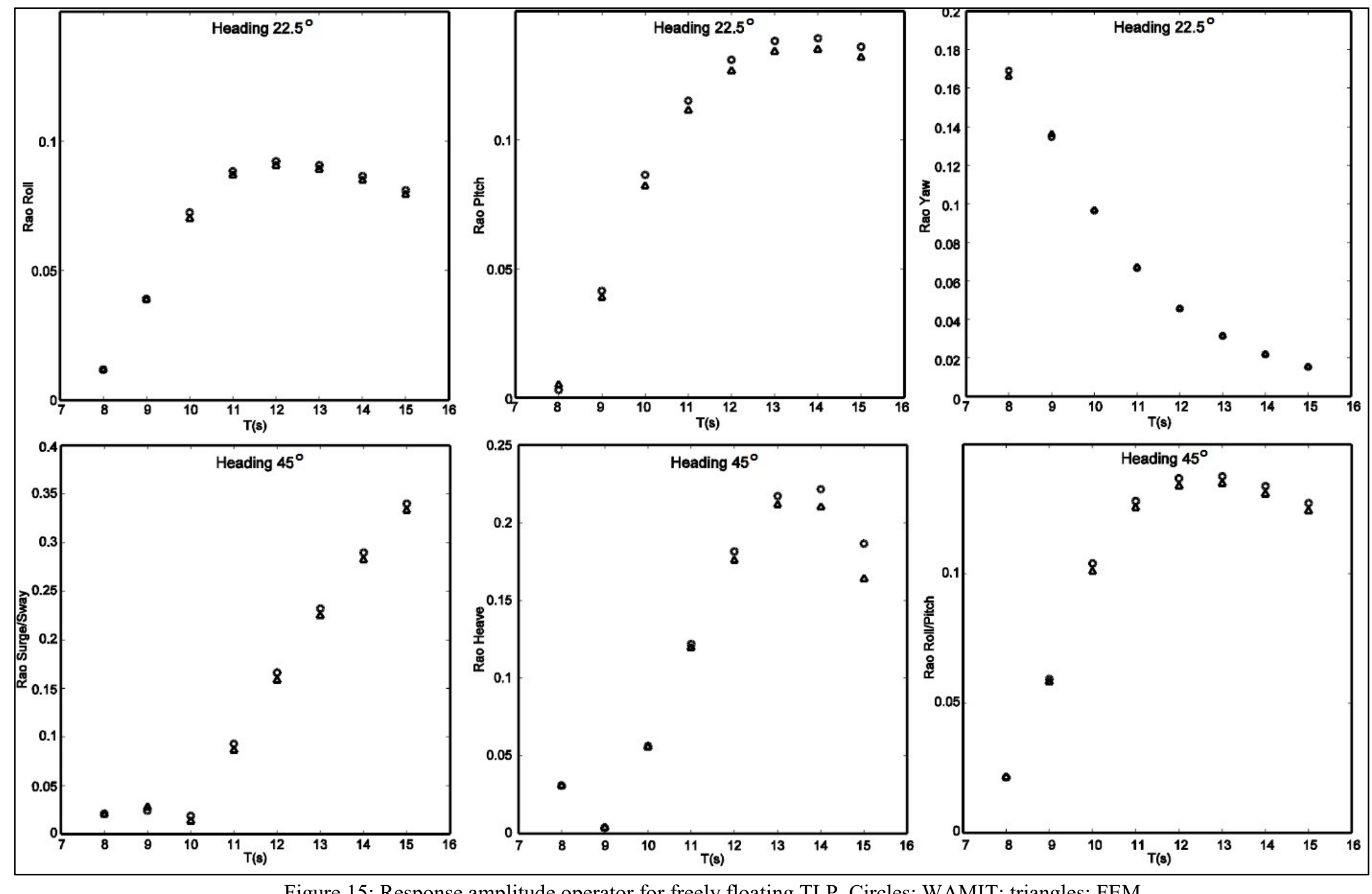

Figure 15: Response amplitude operator for freely floating TLP. Circles: WAMIT; triangles: FEM. 


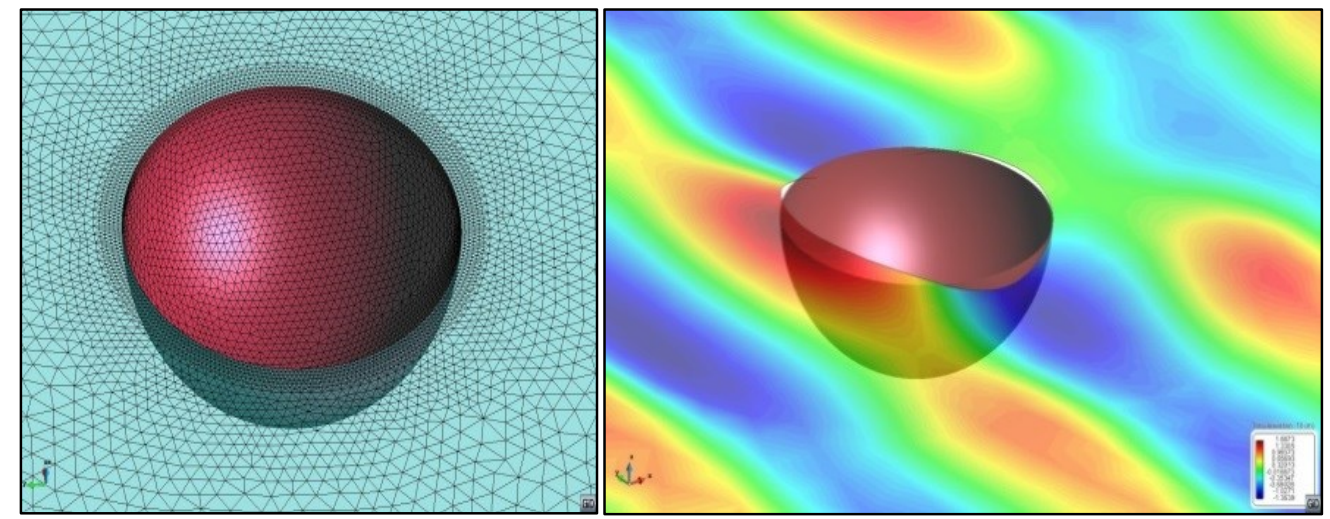

Figure 16: Hemisphere: mesh (left) and wave contours (right)

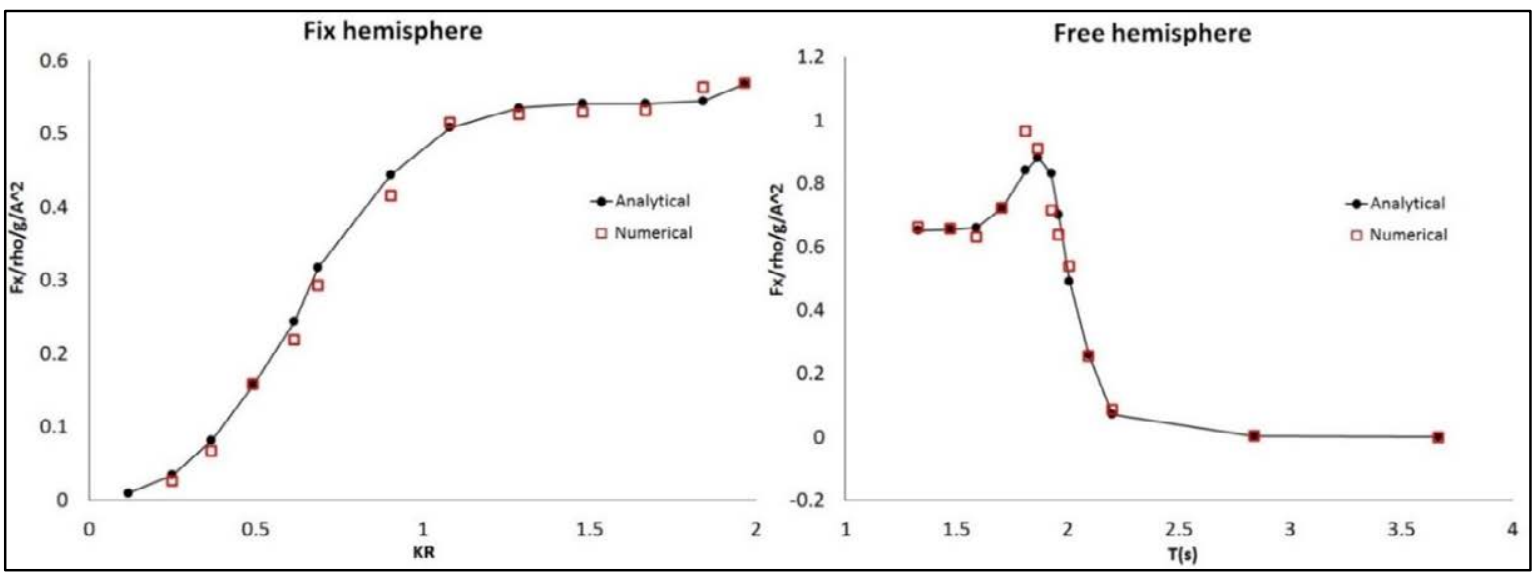

Figure 17: Mean drift forces on Hemisphere

\subsection{Diffraction of second-order waves by semi-submerged horizontal rectangular cylinder}

\subsubsection{Problem description}

This test case deals with the solution to the diffraction problem for second-order surface waves by a semi-submerged horizontal cylinder of rectangular cross section. The boundary-value problem is solved and the results are compared against the analytical solution obtained by the method of matched Eigen function expansions presented in [50]. Horizontal and vertical forces and the moment about the heel of the prismatic cylinder are analyzed for different monochromatic waves. A sketch of the problem under analysis is shown in Figure 18. Relevant geometry parameters are: $\mathrm{h}=1 \mathrm{~m}, \mathrm{~b}=1 \mathrm{~m}$, and $\mathrm{d}=0.2$ m.

The situation considered for analysis is the diffraction of waves by a fixed horizontal cylinder of rectangular cross section. The analysis is undertaken with the following assumptions: the fluid is inviscid and incompressible, the sea bottom and the cylinder are impervious, and the excitation is provided by normally incident plane waves of small amplitude and frequency. Several cases are run 
for different wave periods $(\mathrm{T}=0.897,1.003,1.07,1.16,1.445,2.299,4.17,6.37$ seconds $)$, and the simulation time is about 15 seconds, with an initialization time of 5 seconds. All degrees of freedom are restrained so that the body is completely fixed. Hence, only wave diffraction occurs but not radiation.

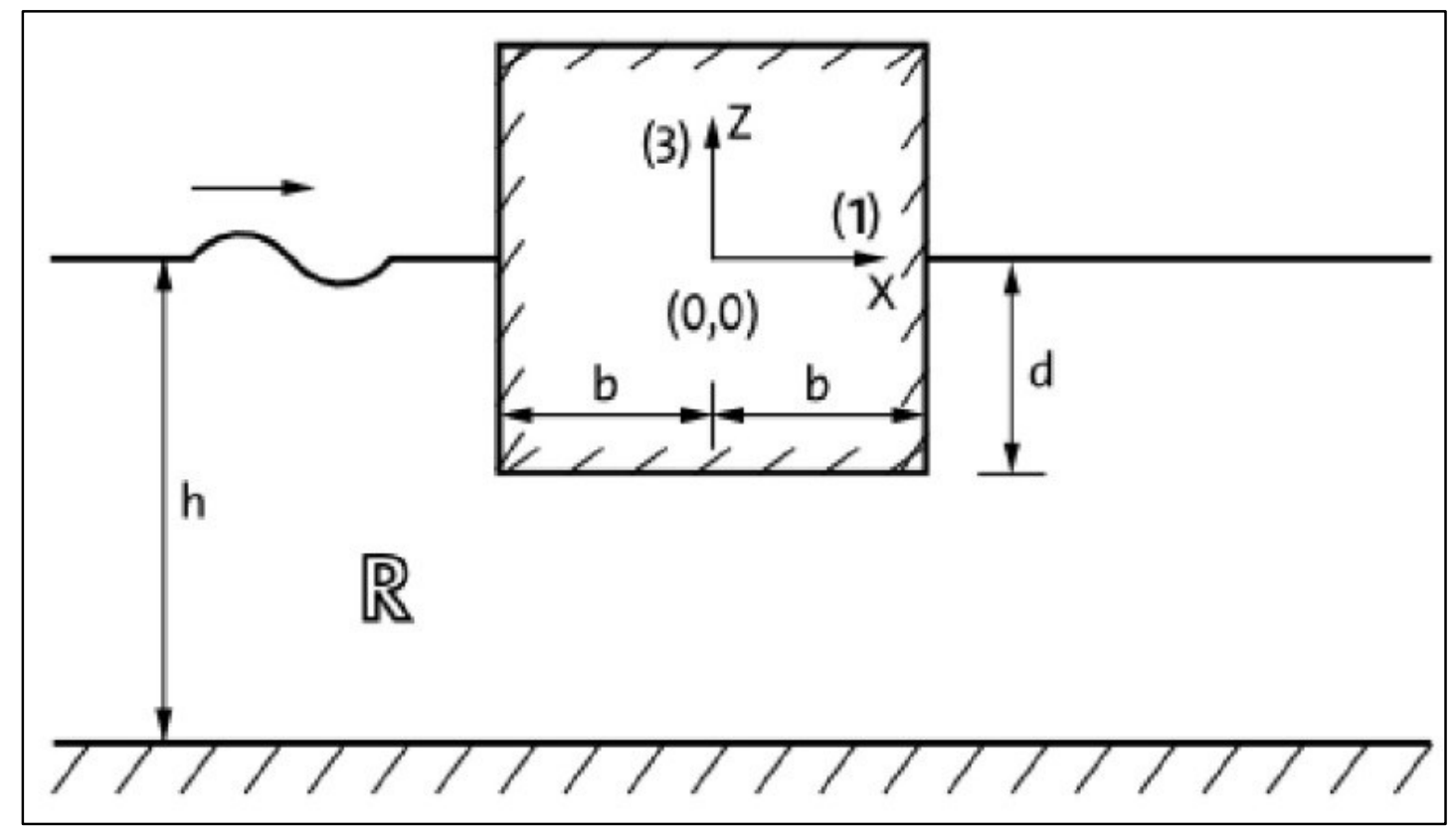

Figure 18: Problem layout

\subsubsection{Mesh generation}

Mesh properties for the present analysis are summarized in Table 6. Figure 19 shows an isometric view of the mesh used for the present analysis at the region close to the surface of the semisubmerged body.

Table 6: Mesh particulars

\begin{tabular}{|c|c|}
\hline Minimum element size & 0.01 \\
\hline Maximum element size & 0.1 \\
\hline Number of elements & 121687 \\
\hline Number of nodes & 22940 \\
\hline
\end{tabular}

\subsubsection{Verification of results}

Figure 20 shows the amplitude of the second-order horizontal and vertical forces, and the moment about y axis, for both the analytical results reported in [50] and the results obtained in this work. The second-order components of the forces (and moments) are normalized respect to the density of the fluid, the gravity, the square of the wave amplitude, and the water depth. Results are plotted against the dimensionless wave number $(\mathrm{kh})$. As it can be observed, a good agreement is obtained for the entire range of wave numbers. 


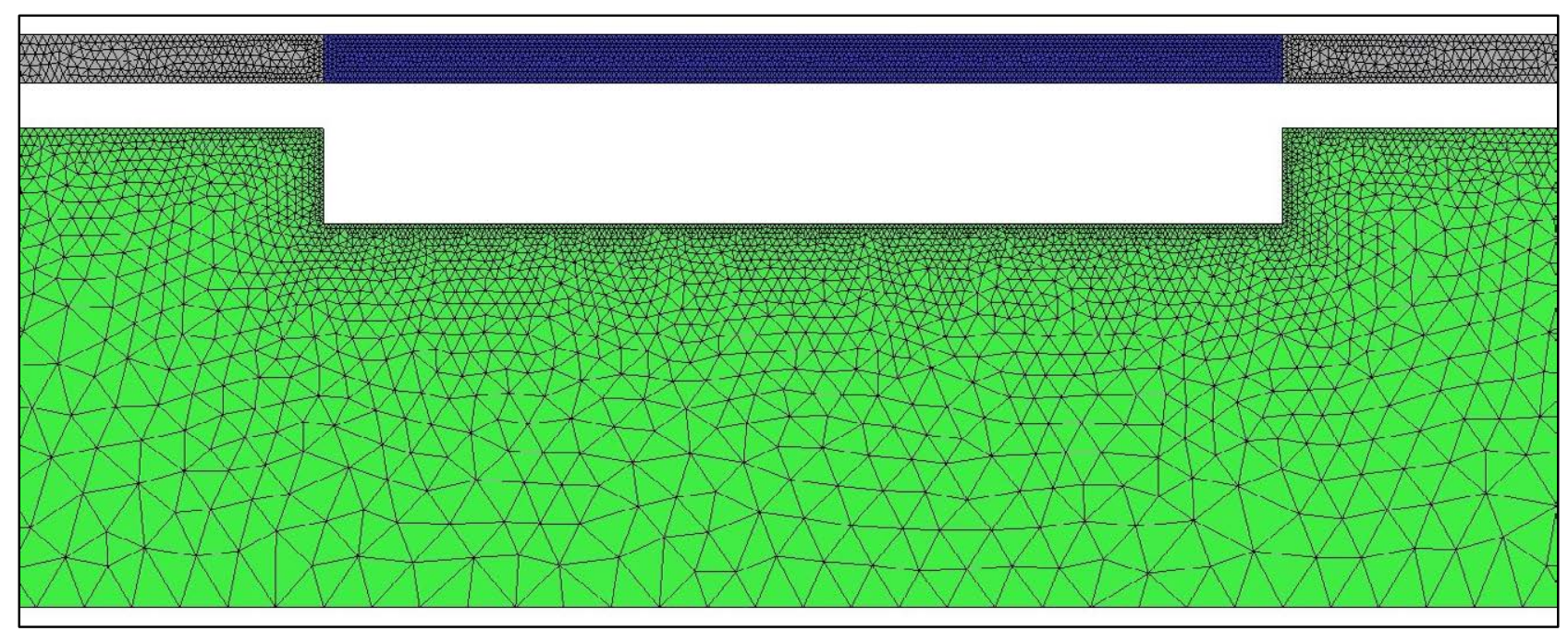

Figure 19: Generated mesh: Top view (up) and front view (down)

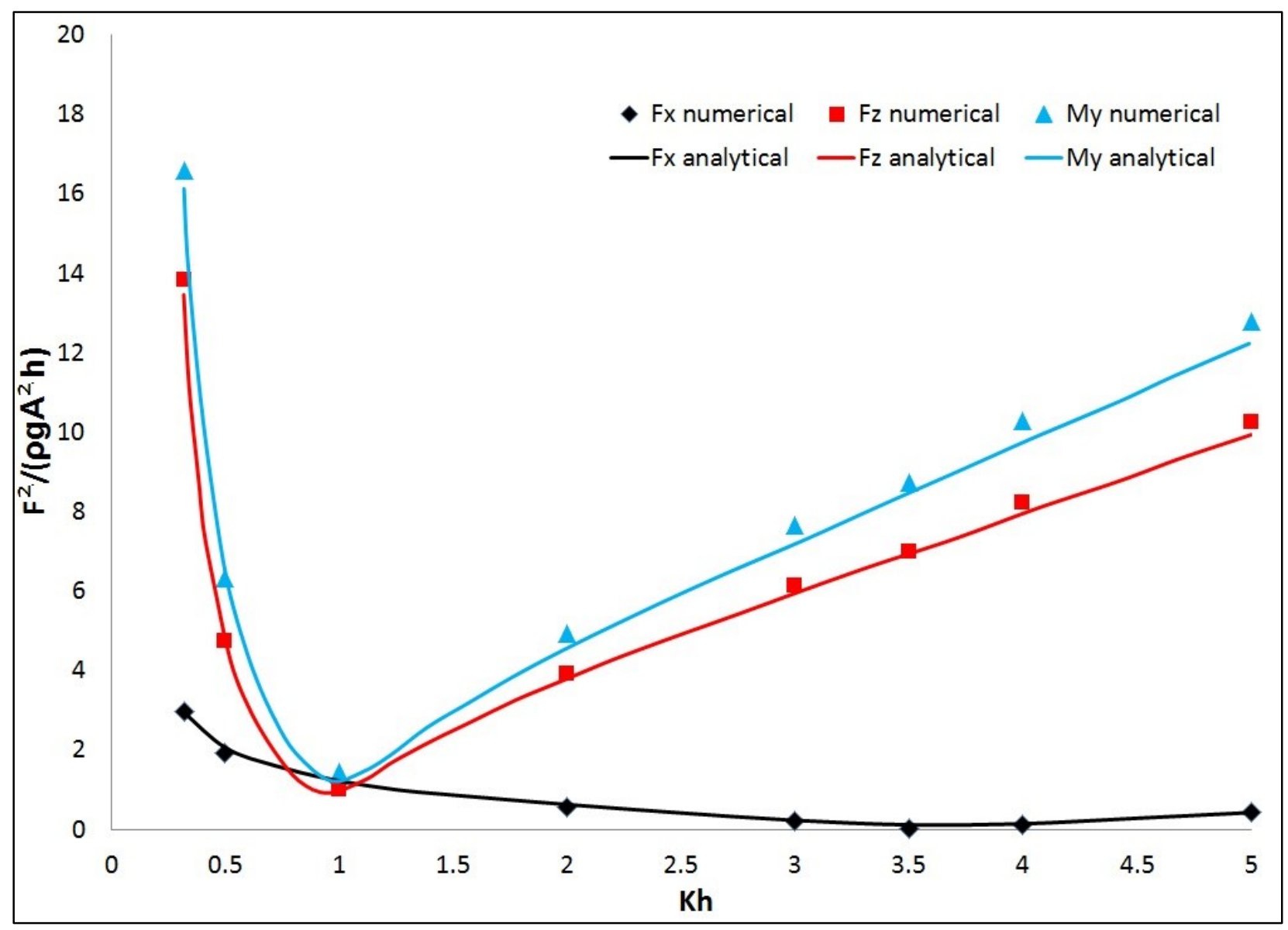

Figure 20: Analytical versus numerical 


\section{Chapter 6. WAVE RESISTANCE: VERIFICATION AND VALIDATION}

\subsection{Introduction}

This chapter aims at the verification of the numerical schemes and the validation of the mathematical models introduced in section 3.2 for wave resistance problems. Firstly, the wave making resistance problem is coped and numerical results are compared against analytical solution for a moving pressure distribution, and against experimental results for the Wigley and Series 60 hulls. Secondly, predictions of added resistance in waves for four modified Wigley hulls are validated against experimental data available.

\subsection{Wave making resistance of an elliptic pressure distribution}

\subsubsection{Problem description}

In 1962, Newman and Poole in [51] derived expressions for the wave resistance of a pressure distribution which is moving with constant forward speed along the free surface of a canal with constant width and depth, and based on a first-order approximation with uniform flow. In particular, an analytical expression for the elliptic pressure distribution was obtained. This expression might be one of the few available for wave making resistance problems, and hence its high value to verify numerical models.

The main particulars of the elliptic pressure distribution used to validate the present model are given in Table 7:

Table 7: Particulars for elliptic pressure distrib
\begin{tabular}{|c|c|}
\hline Length $(\mathbf{L})$ & $1 \mathrm{~m}$ \\
\hline Beam $(\mathbf{B})$ & $0.5 \mathrm{~m}$ \\
\hline Water depth $(\mathbf{H})$ & $5 \mathrm{~m}$ \\
\hline Channel width $(\mathbf{W})$ & $10 \mathrm{~m}$ \\
\hline Free surface pressure $\left(\boldsymbol{P}_{\boldsymbol{f} s}\right)$ & $1 \mathrm{~Pa}$ \\
\hline
\end{tabular}

The wave resistance is obtained integrating the pressure distribution over the free surface as follows:

$$
F_{W}=-\int_{S_{F S}} P_{f s} n_{x} d s
$$


It is assumed that the pressure distribution moves in the $\mathrm{x}$ direction. Then, in order to obtain the wave resistance, it is mandatory to be able to estimate the free surface deformation within the pressure patch.

\subsubsection{Convergence analysis}

Unstructured meshes have been used for the numerical calculations. It has been found that refinement at the edge of the pressure distribution were needed due to the discontinuity in the pressure and its large influence in the free surface deformation, especially at low Froude numbers where the generated waves have smaller wave lengths. Table 8 provides the particulars for each mesh, and Figure 21 shows the finest one.

Table 8: Characteristic mesh sizes

\begin{tabular}{|c|c|c|c|c|}
\hline & $\begin{array}{c}\text { Pressure edge } \\
\mathbf{( m )}\end{array}$ & $\begin{array}{c}\text { Free surface } \\
\text { inside pressure } \\
\mathbf{( m )}\end{array}$ & $\begin{array}{c}\text { Free surface } \\
\text { outside (m) }\end{array}$ & $\begin{array}{c}\text { Volume } \\
\text { underneath (m) }\end{array}$ \\
\hline Mesh 1 & 0.002 & 0.02 & 0.02 & 0.05 \\
\hline Mesh 2 & 0.003 & 0.03 & 0.03 & 0.075 \\
\hline Mesh 3 & 0.004 & 0.04 & 0.04 & 0.1 \\
\hline Mesh 4 & 0.006 & 0.06 & 0.06 & 0.15 \\
\hline Mesh 5 & 0.008 & 0.08 & 0.08 & 0.20 \\
\hline
\end{tabular}

Wave resistance was calculated for all meshes at Froude number $\mathrm{Fr}=0.2$, and for the streamline and FEM-SUPG schemes presented in sections 3.2.3 and 3.2.4 respectively. Table 9 provides the values of the relative errors. Figure 22 shows the free surface elevation computed for $\mathrm{Fr}=0.2$ with the FEM-SUPG and Mesh1.

Table 9: Wave resistance $R_{W}^{*}=\rho g F_{W} /\left(P_{f s}^{2} B\right)$ at Fr=0.2
\begin{tabular}{|c|c|c|c|c|c|c|}
\hline \multirow{2}{*}{ Analytical $\boldsymbol{R}_{W}^{*}=\mathbf{0 . 1 8 4 0}$} & Mesh 5 & Mesh 4 & Mesh 3 & Mesh 2 & Mesh 1 \\
\hline \multirow{2}{*}{ Streamline } & $R_{W}^{*}$ & 1.550 & 1.640 & 1.730 & 1.780 & 1.820 \\
\cline { 2 - 8 } & Error & $15.77 \%$ & $10.88 \%$ & $5.99 \%$ & $3.27 \%$ & $1.10 \%$ \\
\hline \multirow{2}{*}{ FEM-SUPG } & $R_{W}^{*}$ & 1.643 & 1.710 & 1.775 & 1.795 & 1.820 \\
\cline { 2 - 7 } & Error & $10.74 \%$ & $7.08 \%$ & $3.54 \%$ & $2.46 \%$ & $1.10 \%$ \\
\hline
\end{tabular}

\subsubsection{Verification}

The wave making resistance has been calculated for several Froude numbers for the finest mesh using the streamline and FEM-SUPG algorithm. Table 10 provides the numeric results obtained, as well as the analytical ones. It can be observed that the numerical results are able to reproduce the analytical ones with small errors. 


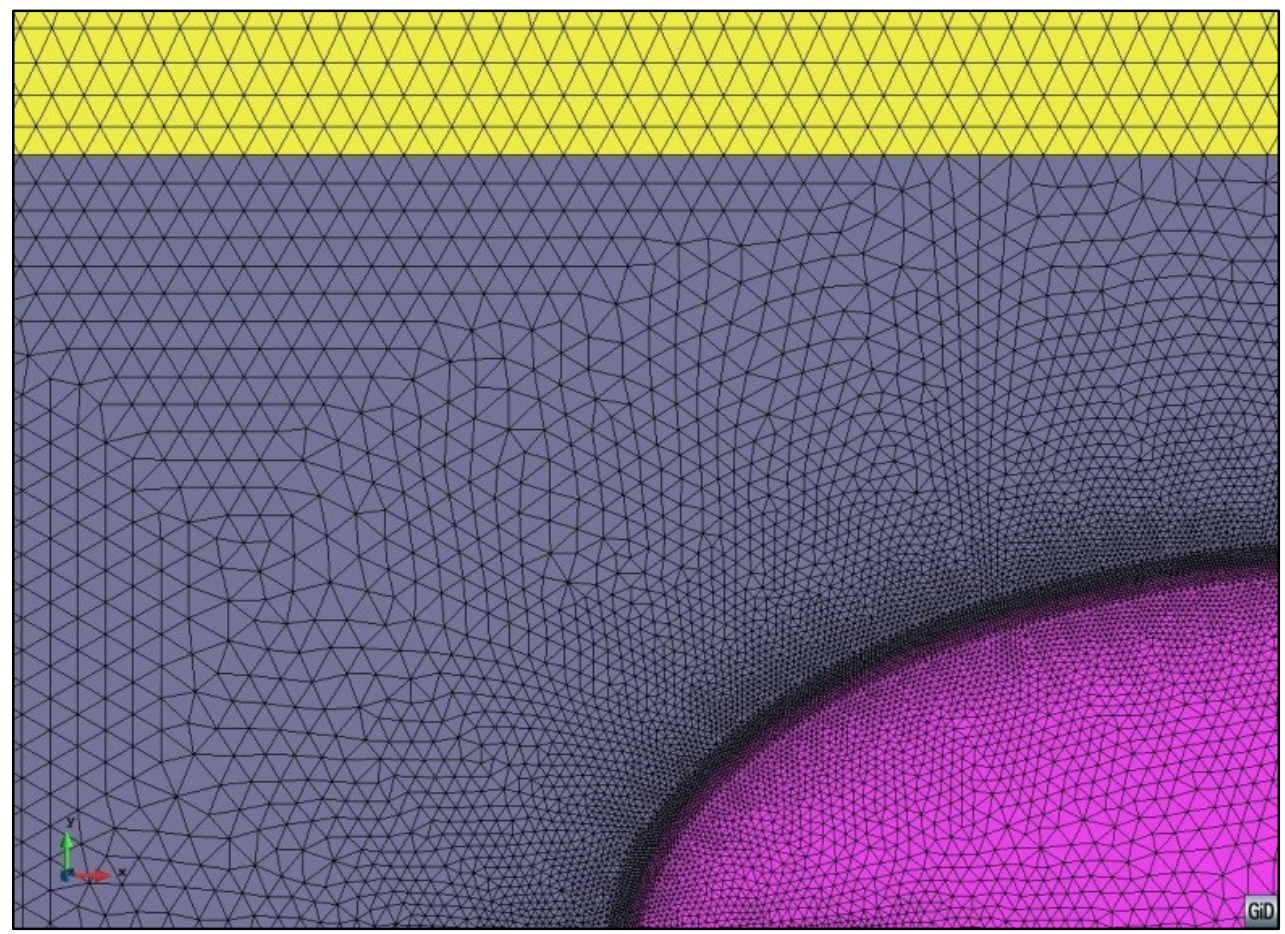

Figure 21:Finest Mesh for elliptic pressure patch

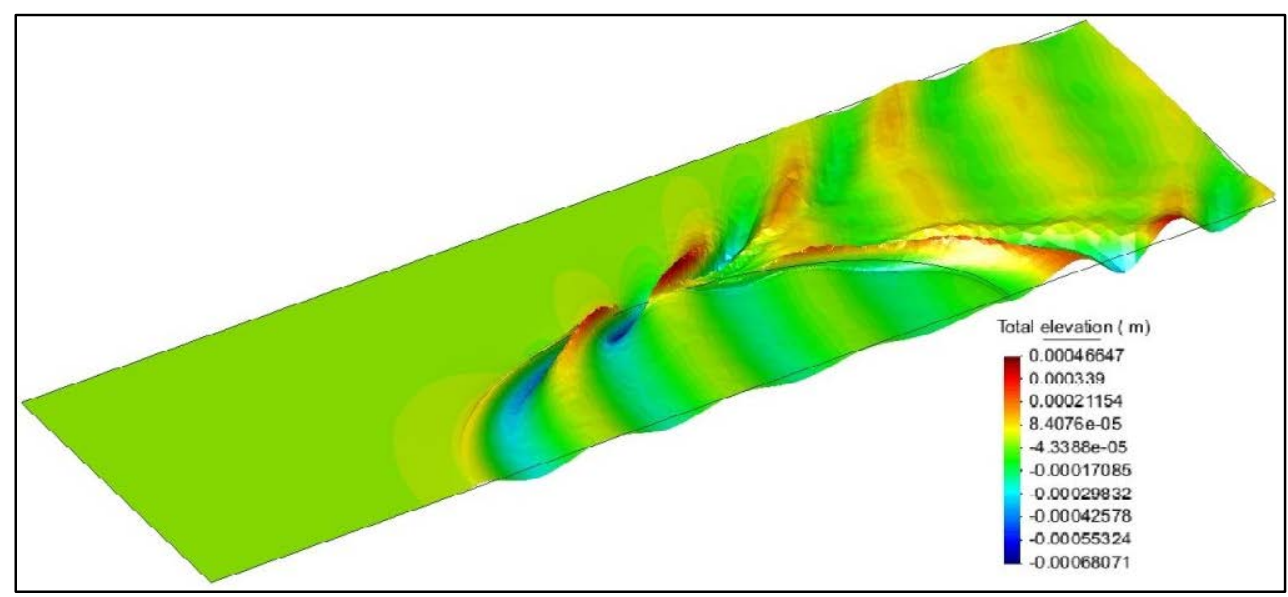

Figure 22: Free surface elevation for elliptic pressure distribution at $\mathrm{Fr}=0.2$

Table 10: Wave resistance for elliptic pressure distribution

\begin{tabular}{|c|c|c|c|}
\hline Fr & Streamline & FEM-SUPG & Analytical \\
\hline $\mathbf{0 . 2}$ & 1.820 & 1.82 & 1.84 \\
\hline $\mathbf{0 . 3}$ & 2.177 & 2.16 & 2.18 \\
\hline $\mathbf{0 . 4}$ & 1.67 & 1.65 & 1.64 \\
\hline $\mathbf{0 . 5}$ & 2.73 & 2.66 & 2.66 \\
\hline
\end{tabular}

\subsection{Wave making resistance of a Wigley hull}

\subsubsection{Case study}

The wave making resistance of a Wigley hull is analyzed. Simulations have been performed considering the non-linear free surface condition, the Neumann-Kelvin, and the double-body 
linearization (see 3.1.5 and 3.1.6). The streamline and FEM-SUPG schemes have been used for integrating the free surface boundary conditions. Wave resistance coefficients with and without second-order correction have been obtained.

A standard Wigley hull of length $\mathrm{L}=1 \mathrm{~m}$ and breadth $\mathrm{B}=0.1 \mathrm{~m}$ was used. An unstructured mesh was generated with an element size around the ship of $0.01 \mathrm{~m}$. The mesh generated consists of 84351 nodes and 476083 tetrahedral elements (see Figure 23).

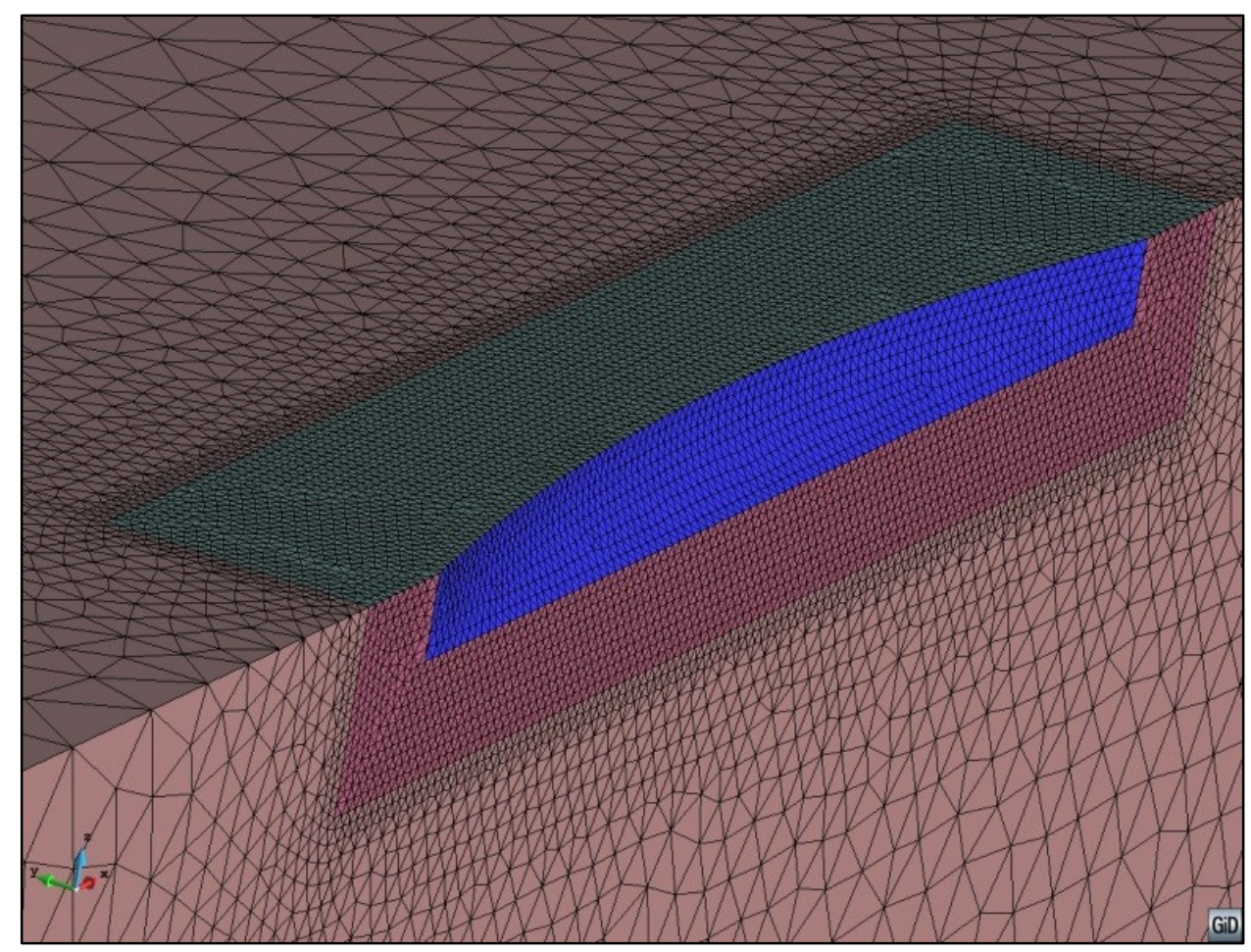

Figure 23: Mesh generated for Wigley hull

\subsubsection{Second-order correction and flow linearizations}

Figure 24 compares the first-order and second-order wave resistance coefficients $\left(\mathrm{C}_{\mathrm{w}}=\right.$ $\left.\mathrm{R}_{\mathrm{w}} /\left(0.5 \rho \mathrm{SU}^{2}\right)\right)$, in the case of a fixed model, obtained for the non-linear and linearized formulations. It can be easily observed that the increase of resistance due to the second-order correction term is noticeable.

On the one hand, the Neumann-Kelvin linearization leads to smaller values of wave resistance when compared to the non-linear and double-body approaches. On the other hand, the double-body and non-linear approaches predict similar results up to $\mathrm{Fr}=0.3$. For larger values of Froude number, when using the streamline scheme, the double-body is not as close to the non-linear as when using the SUPG scheme. 


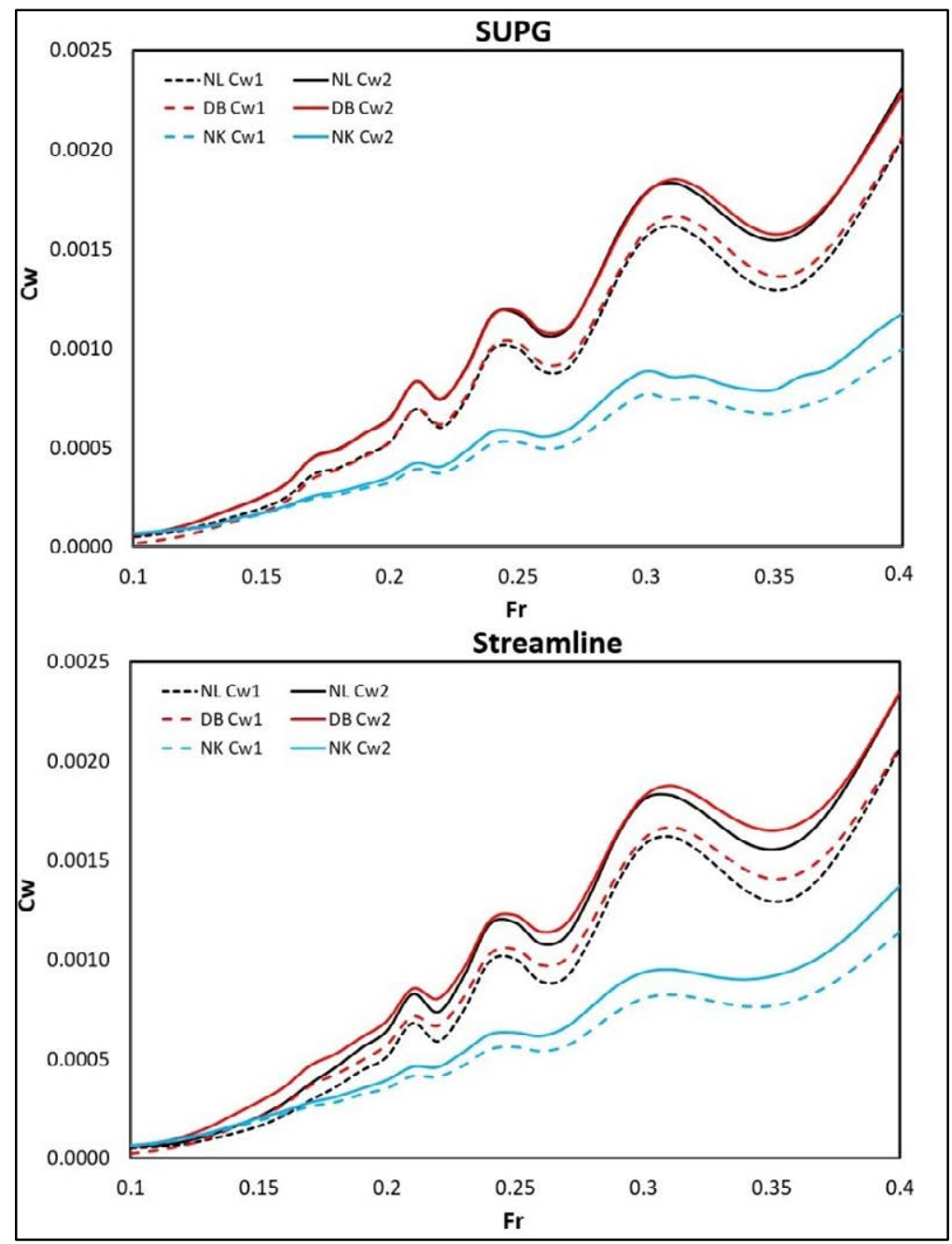

Figure 24: Wave resistance for Wigley hull: Non-linear (NL), Double-body (DB), and Kelvin (K). First-order (Cw1), and Second-order $(\mathrm{Cw})$.

\subsubsection{Validation}

Figure 25 compares the numerical results obtained in this thesis with several experimental results: IITC 84 (collection of experimental data) [26], Shearer and Cross [52], University of Iowa, University of Tokyo (UT), and the Ship Research Institute of Japan (SRI). The results from Iowa, UT and SRI can be found in [53]. Experimental data shows large dispersion and this might be mainly due to two main reasons: the uncertainty when carrying out model testing, and due to the consideration of a constant form factor with speed when separating wave making resistance from frictional resistance.

Taking into account the large dispersion of the experimental data, a fair agreement between the numerical and the experimental results. This agreement is better for the higher Froude number, as expected, since lower Froude numbers require a much finer discretization in order to reproduce the shorter waves generated. Figure 26 compares the wave profiles at the water line obtained numerically against experimental results, and a good agreement is also found. 


\subsection{Wave making resistance of a Series 60 hull}

\subsubsection{Case study}

The wave making resistance of a Series 60 hull is analyzed. Simulations have been performed considering the non-linear free surface condition, the Neumann-Kelvin, and the double-body linearization (see 3.1.5 and 3.1.6). The streamline and FEM-SUPG schemes have been used for integrating the free surface boundary conditions. Wave resistance coefficients with and without second-order correction have been obtained.

A standard Series 60 hull of length $\mathrm{L}=1 \mathrm{~m}$ and breadth $\mathrm{B}=0.130 \mathrm{~m}$ with block coefficient $\mathrm{C}_{\mathrm{b}}=0.6$ was used. An unstructured mesh, with an element size around the ship of $0.01 \mathrm{~m}$, was generated such the mesh consists of 130050 nodes and 739099 tetrahedral elements (see Figure 27).

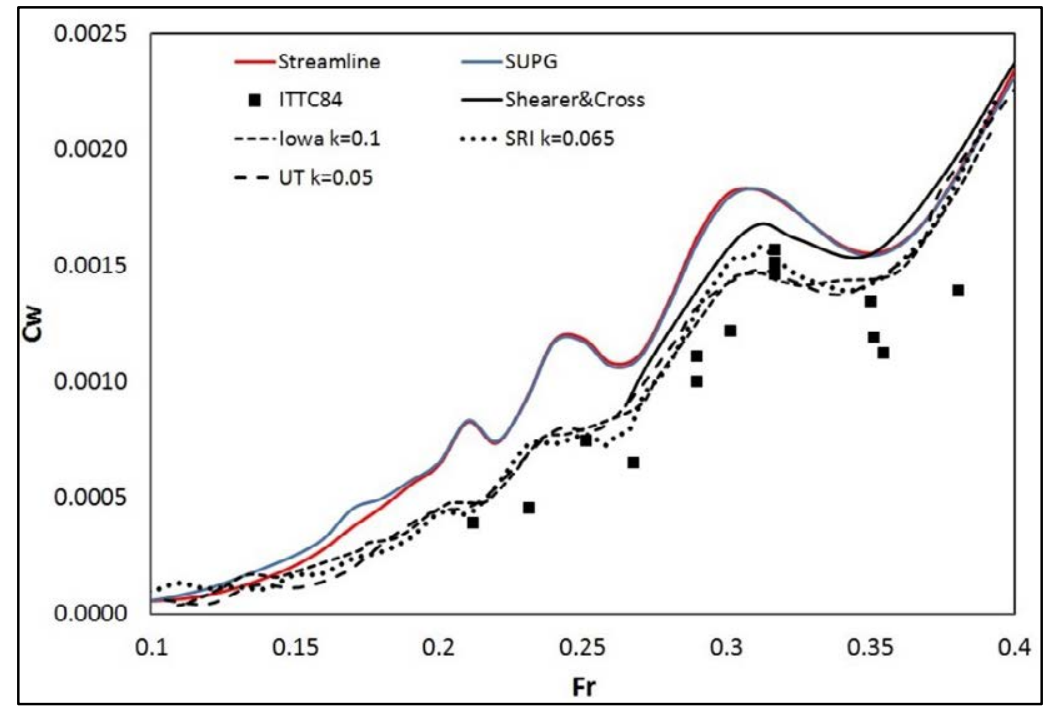

Figure 25: Streamline and SUPG versus experimental results.

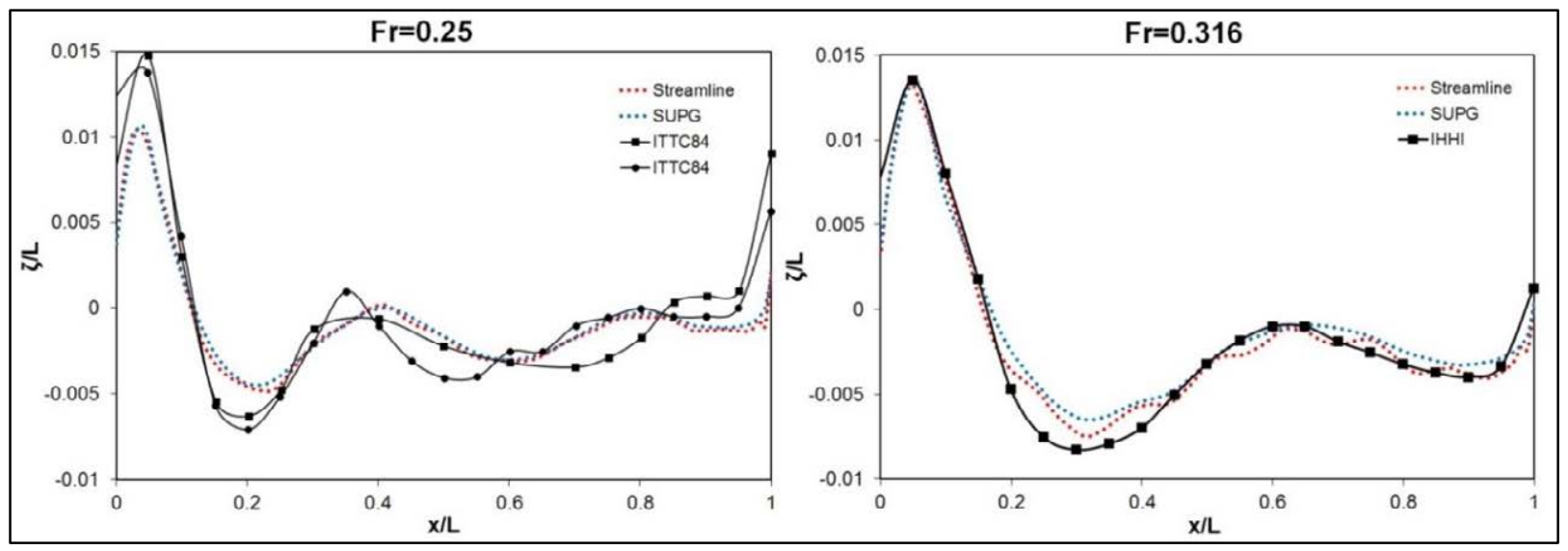

Figure 26: Wave profile over Wigley hull. 


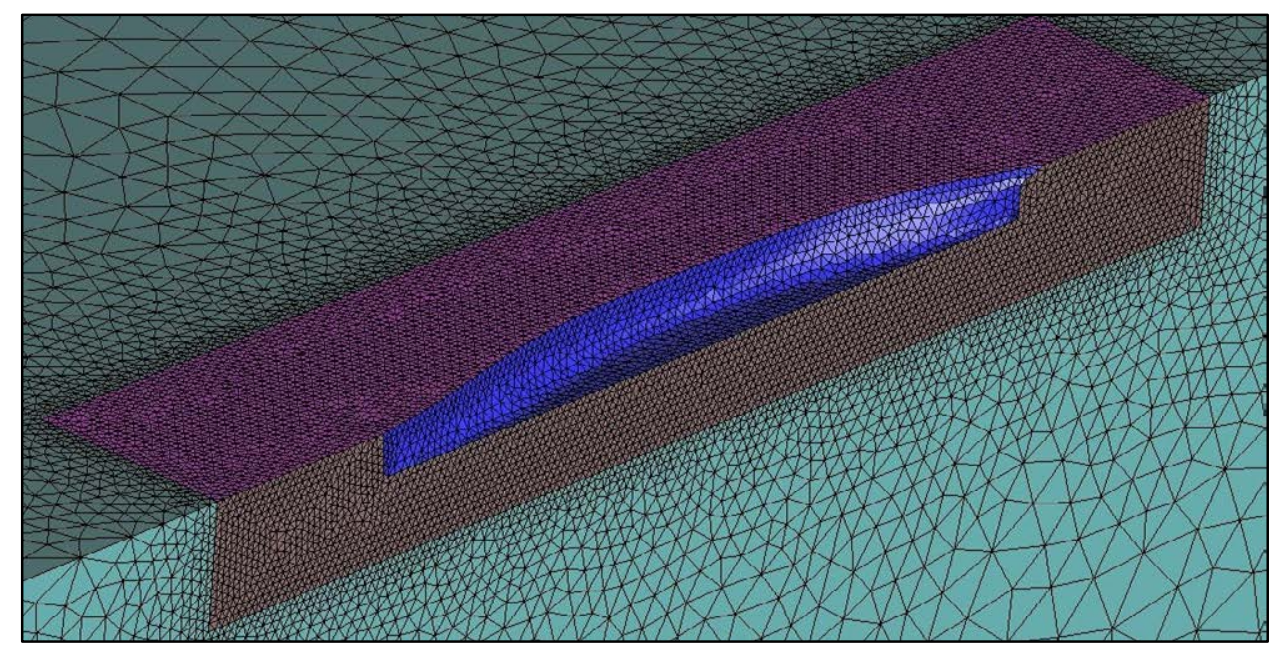

Figure 27: Series 60 hull mesh.

\subsubsection{Second-order correction and flow linearization}

Figure 28 compares the first-order and second-order wave resistance coefficients, in the case of a fixed model, obtained for the different free surface formulations. It can be easily observed that the increase of resistance due to the second-order term is noticeable.

Regarding the use of the Neumann-Kelvin or the double-body linearization, none of them are close to the non-linear approach solution. Hence, no linearization is recommended for hulls not as slender as the Wigley hull. In particular, when using the Neumann-Kelvin linearization, the deviation from the non-linear results is very important, probably due to the fact that in the area close to the ship the approximation of streamlines by straight lines is not appropriated.

\subsubsection{Validation}

Figure 29 compares the numerical results obtained in this work with several experimental results: IITC 84 (collection of experimental data) [26], University of Tokyo (UT), and the IshikawajimaHarima Heavy Industries Co., Ltd. (IHHI) in Japan. As for the Wigley hull, experimental results show large dispersion across different facilities. Overall, the numerical results are within the range of dispersion of the experimental ones.

Figure 30 compares the experimental and numerical wave profiles over the hull for $\mathrm{Fr}=0.316$ and a good agreement is found among them. 


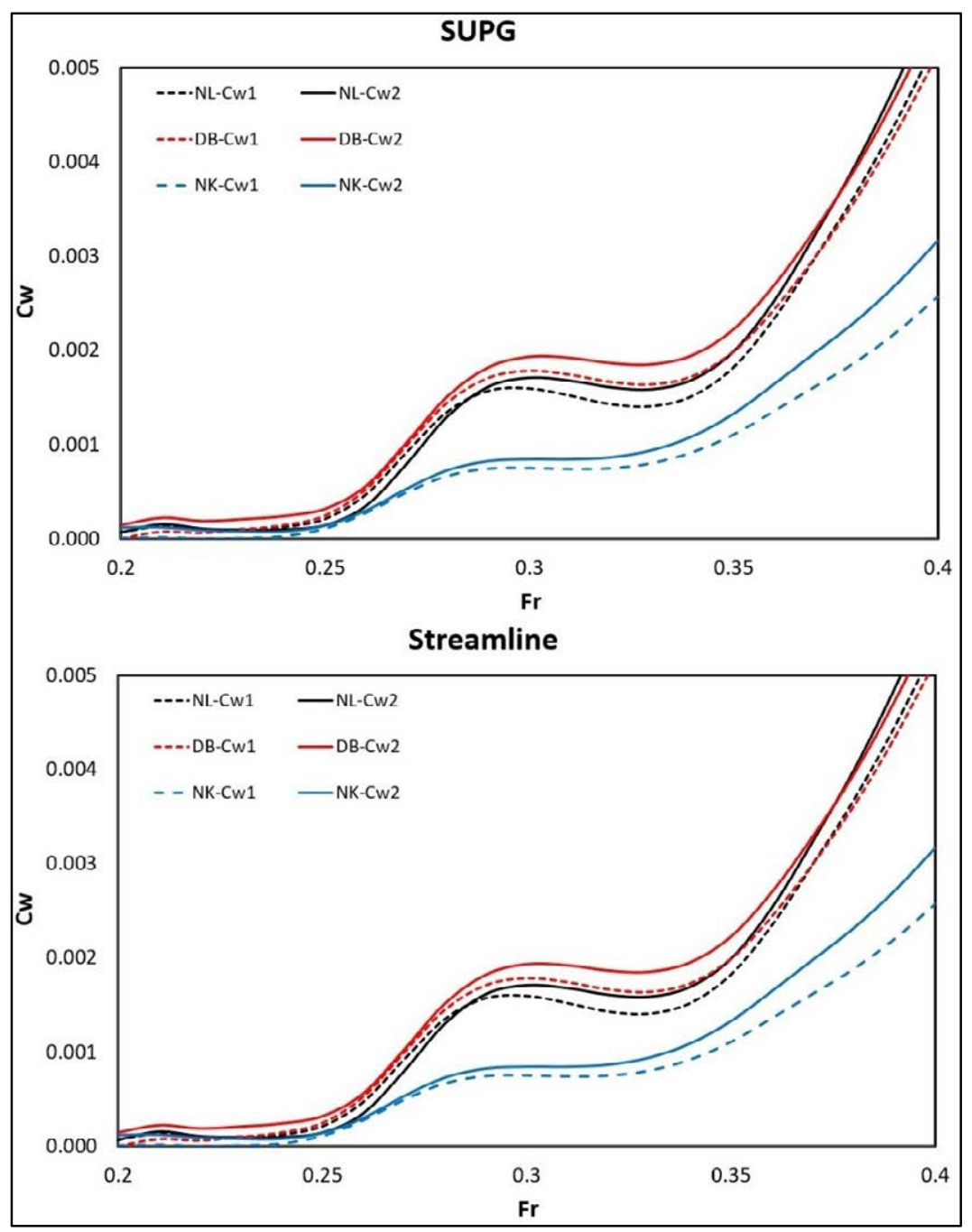

Figure 28: Wave resistance for Series 60 hull: Non-linear (NL), Double-body (DB), and Neumann-Kelvin (NK). Firstorder ( $\mathrm{Cw} 1)$, and second-order $(\mathrm{Cw})$.

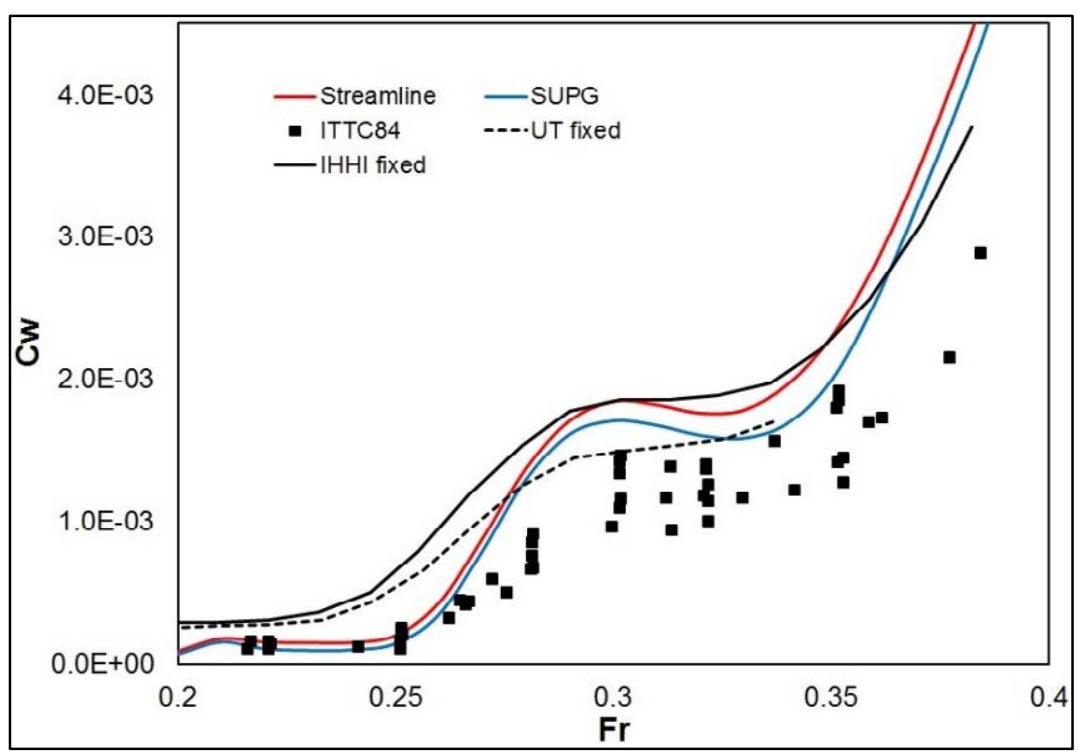

Figure 29: Comparison with experimental results. 


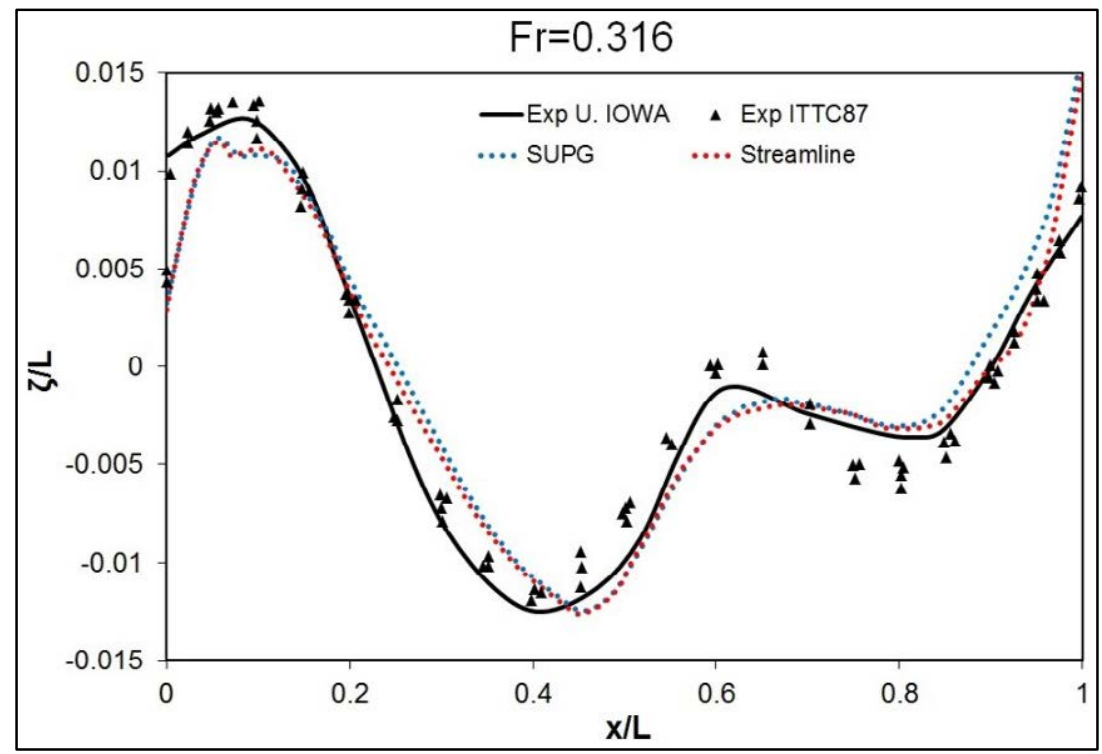

Figure 30: Wave profile over Series 60 hull

\subsection{Added resistance in waves of four modified Wigley hulls}

\subsubsection{Case study}

In [30], Journée carried out an extensive model test comparing with four modified Wigley hulls and measured the added resistance in waves. In this section, computed added resistance is compared to that obtained in [30] for the four modified Wigley hull models. The main particulars of these models are given in Table 11.

Table 11: Modified Wigley hulls particulars.

\begin{tabular}{|c|c|c|c|c|}
\hline & Wigley I & Wigley II & Wigley III & Wigley IV \\
\hline $\begin{array}{c}\text { Amidships section } \\
\text { coefficient, Cm }\end{array}$ & 0.9090 & 0.9090 & 0.6667 & 0.6667 \\
\hline Length to breadth ratio, L/B & 10 & 5 & 10 & 5 \\
\hline Length, L (m) & 1 & 1 & 1 & 1 \\
\hline Breadth, B (m) & 0.1 & 0.2 & 0.1 & 0.2 \\
\hline Draught, d (m) & 0.0625 & 0.0625 & 0.0625 & 0.0625 \\
\hline Displacement (m3) & 0.003504 & 0.007008 & 0.002889 & 0.005778 \\
\hline KG & 0.05667 & 0.0625 & 0.05667 & 0.0625 \\
\hline $\begin{array}{c}\text { Radius of inertia for pitch, } \\
\boldsymbol{k}_{y y} \text { (m) }\end{array}$ & 0.25 & 0.25 & 0.25 & 0.25 \\
\hline $\begin{array}{c}\text { Pitch damping (N/(m/s)) } \\
\text { damp }\end{array}$ & $0.02 \sqrt{4 I_{y y} K_{55}}$ & $0.10 \sqrt{4 I_{y y} K_{55}}$ & $0.02 \sqrt{4 I_{y y} K_{55}}$ & $0.02 \sqrt{4 I_{y y} K_{55}}$ \\
\hline
\end{tabular}

\subsubsection{Validation}

In order to estimate the added resistance $R_{a w}$, the resistance in still water is subtracted from to the average resistance value in waves, obtained via the mathematical model described in 3.3.6. The dimensionless wave resistance is then obtained as: 


$$
R_{a w}^{*}=\frac{R_{a w}}{\rho g A^{2} B^{2} / L},
$$

where $\mathrm{A}$ is the wave amplitude.

Simulations were carried out with the streamlines and FEM-SUPG schemes, and both of them with the non-linear approximations. Pitch damping was introduced in order to calibrate pitch movements around resonance, otherwise excessive pitch movement around resonance would modify the added resistance estimation.

Figure 31 shows a snapshot of the wave pattern generated by the Wigley III hull at $\mathrm{Fr}=0.3$ advancing against a monochromatic wave with wave length the length of the hull. Figure 32 shows the hull geometries and mesh sizes used on the hull and the near free surface.

Figure 33-Figure 36 show the heave and pitch RAOs, as well as $\mathrm{R}_{\mathrm{aw}}^{*}$, versus the dimensionless wavelength for the four modified Wigley hull models. Heave movements were measured at $0.075 \mathrm{~m}$ from the gravity center towards the stern.

Overall, results obtained with the streamline and the SUPG are quite similar to each other. Only in a few figures some slight deviation is observed from each other.

Regarding the experimental results, trends are well defined in general, although in some cases some scattering of results is observed. Numerical results for RAOS and $\mathrm{R}_{\mathrm{aw}}^{*}$ follow reasonably well the experimental data, although the goodness of the fitting depends on each case. Resonance frequencies are recovered well, although peak values of added resistance are often underestimated.

In [30] it was reported that in some case studies (for instance Wigley II at $\mathrm{Fr}=0.3$ and $\mathrm{Fr}=0.4$ ) it was not possible to stabilized the experiments, and therefore no results were obtained in those cases, which gives an idea on how complex it is to carry out this sort of studies. Moreover, added resistance in waves is a second-order force and quite small when compared to first-order forces, which makes very complicated measuring and separating those quantities. In fact, quite large models are needed to be able to measure second order forces, which is not the case in [30] where the model length was $3 \mathrm{~m}$. Since experimental data have significant uncertainties, implying that such results must be taken as qualitative rather than quantitative values. 


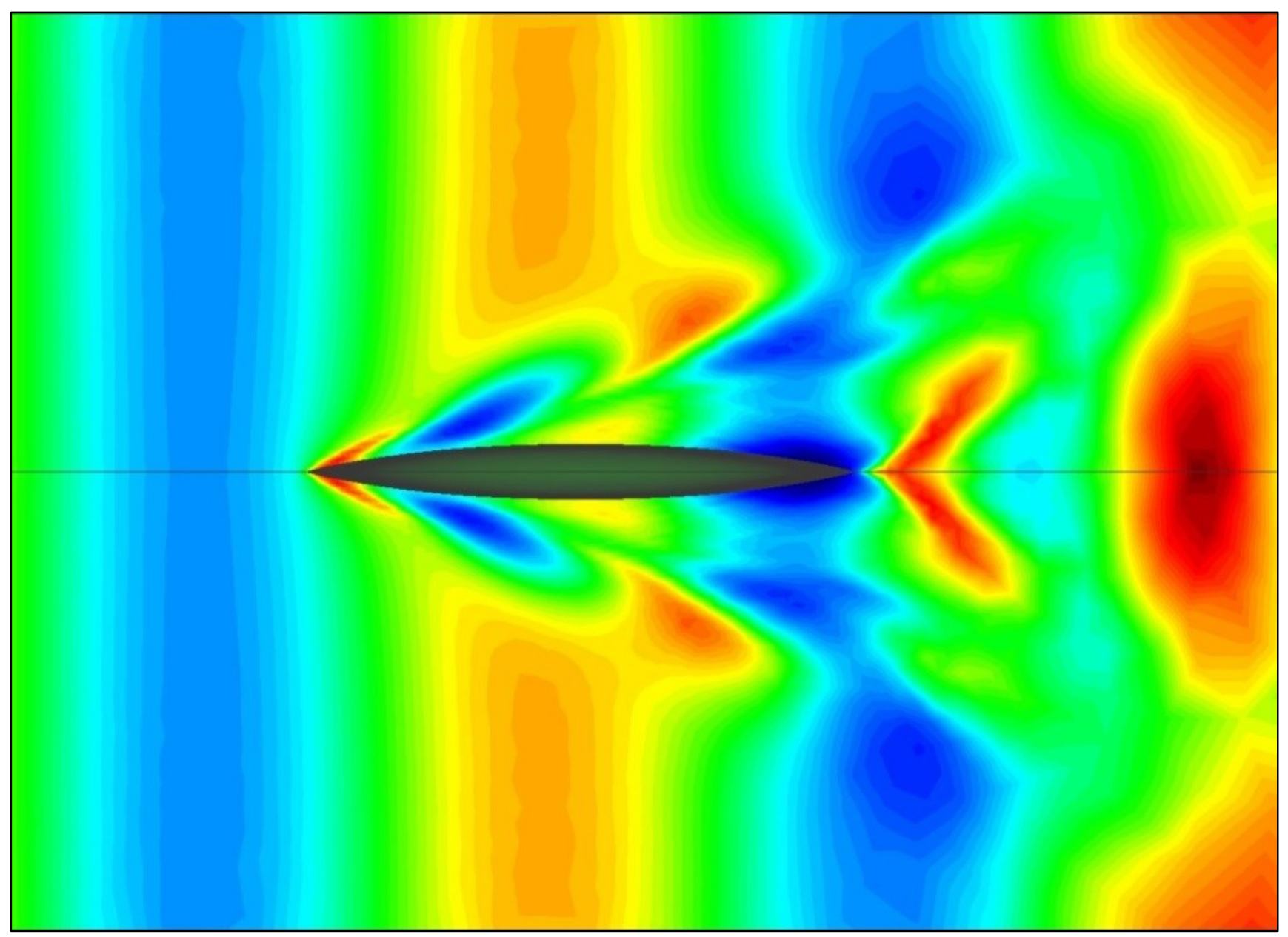

Figure 31: Wave pattern for Wigley III. Fr=0.3. $\lambda_{\mathrm{w}} / \mathrm{L}=1$. 


$$
\div
$$




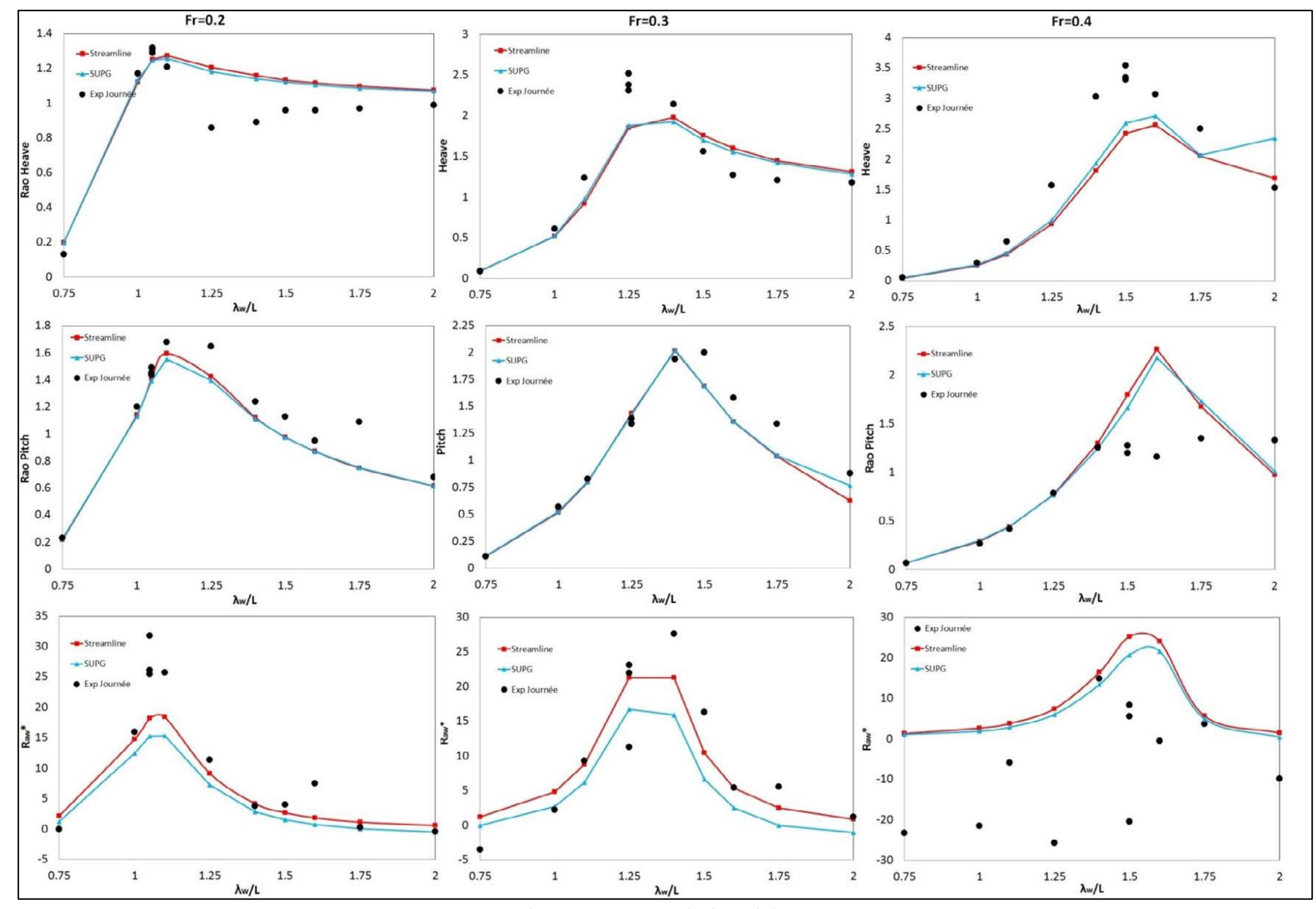

Figure 33: RAOs and $R_{a w}^{*}$ Wigley I. 


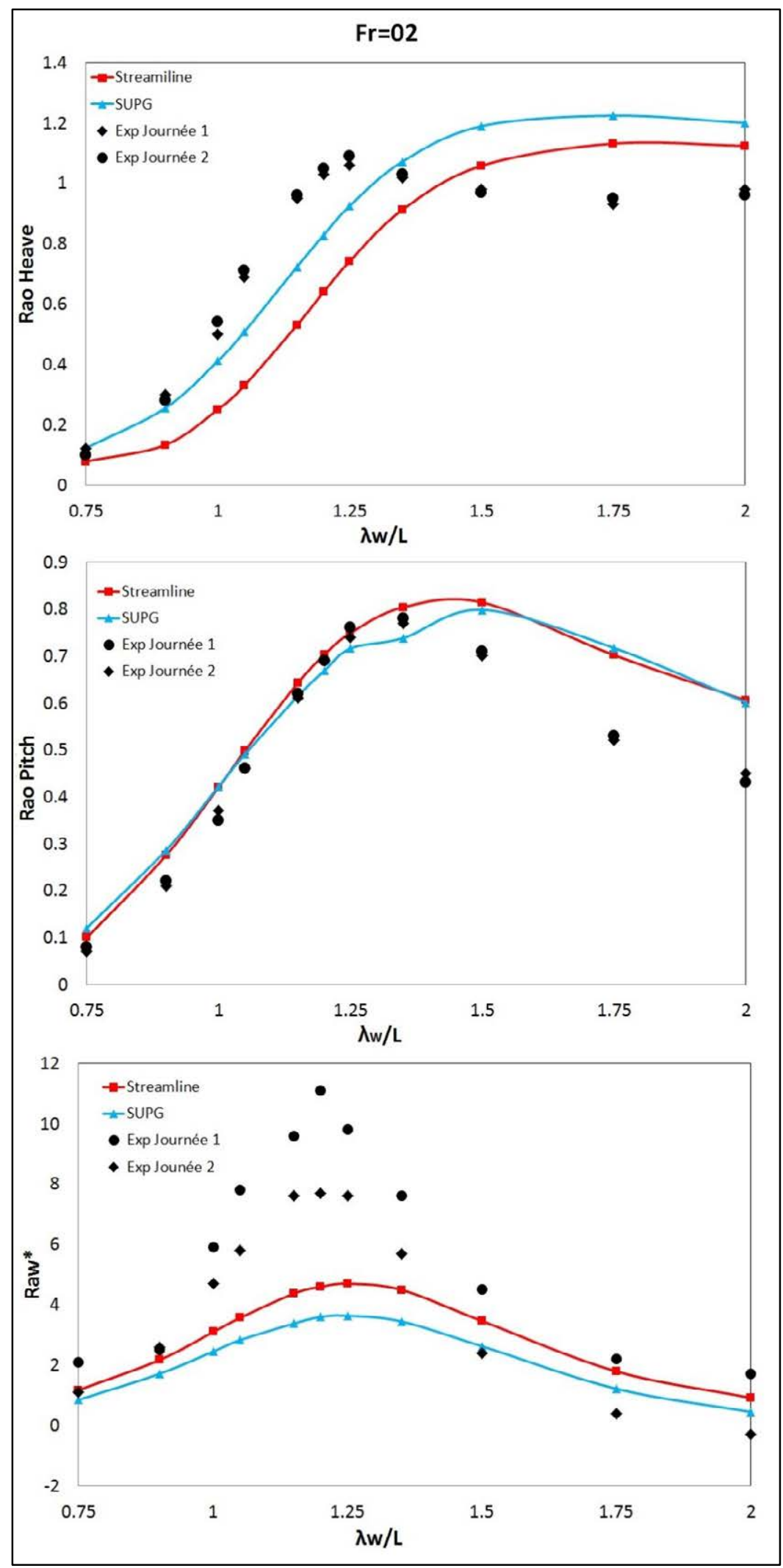

Figure 34: RAOs and $R_{a w}^{*}$ for Wigley II. 


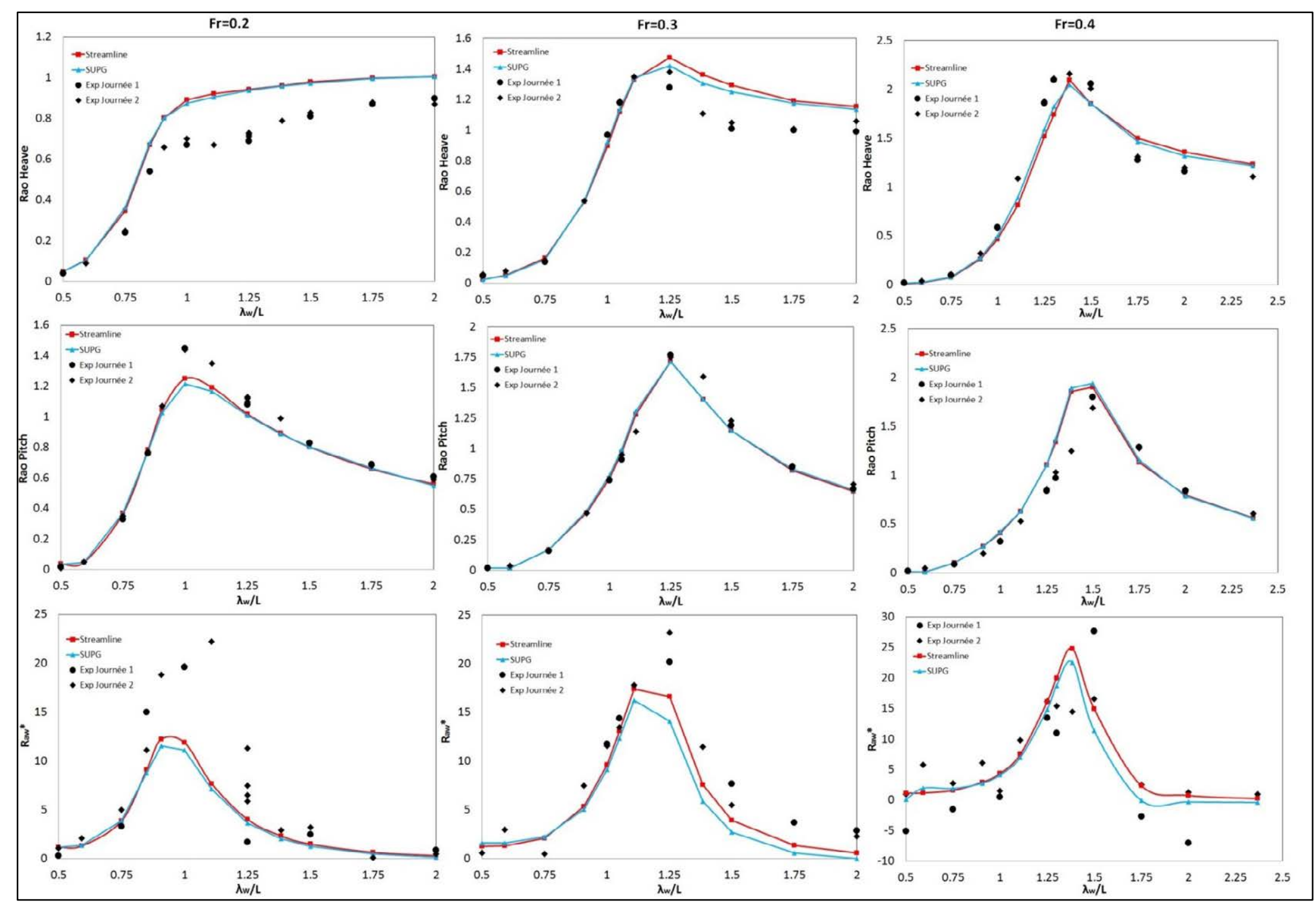

Figure 35: RAOs and $R_{a w}^{*}$ for Wigley III. 


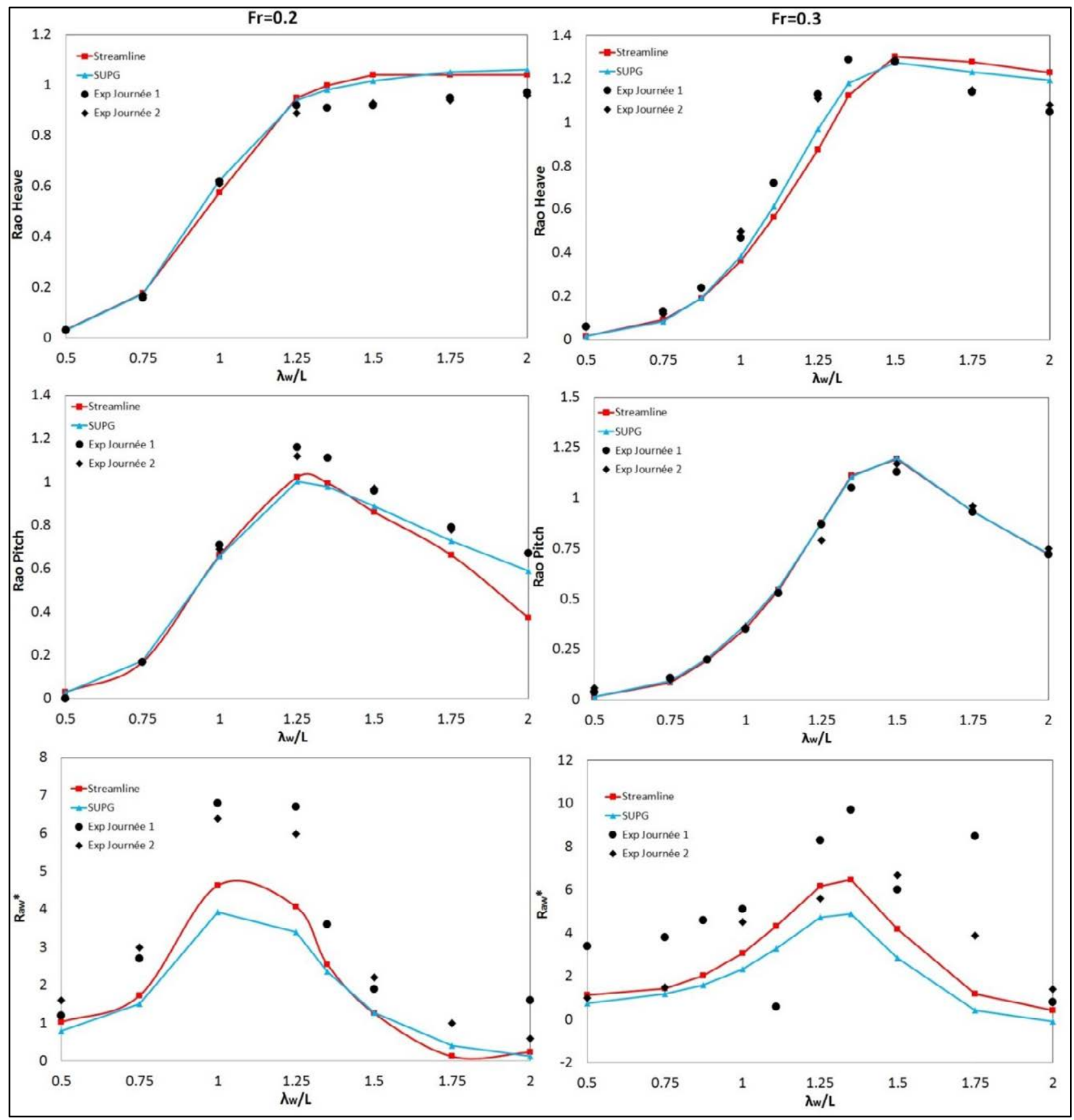

Figure 36: RAOs and $R_{a w}^{*}$ for Wigley IV. 


\section{Chapter 7. BODY DYNAMICS: VERIFICATION}

\subsection{Introduction}

The aim of this chapter is to show the details of how body links are introduced within the body dynamics solver for a simple case study. Numerical results obtained are discussed.

\subsection{Case study: 2D Rotating-ring pendulum}

In this section, a ring performing an oscillating motion is analyzed to verify the accuracy of the body dynamics solver along with body links. The gravity center of the ring is located at its geometrical center and one meter below the center of rotation (located at the origin of coordinates). The ring is one meter in radius, has a mass of $1 \mathrm{~kg}$, and all its mass is assumed to be concentrated around its perimeter. It is given an initial rotation of $90^{\circ}$ around the $\mathrm{Y}$ axis, which translates the gravity center from point $(0,0,-1)$ to point $(-1,0,0)$. Then the ring is left free to oscillate under the action of gravity. Figure 37 shows the rotating ring and its initial position.

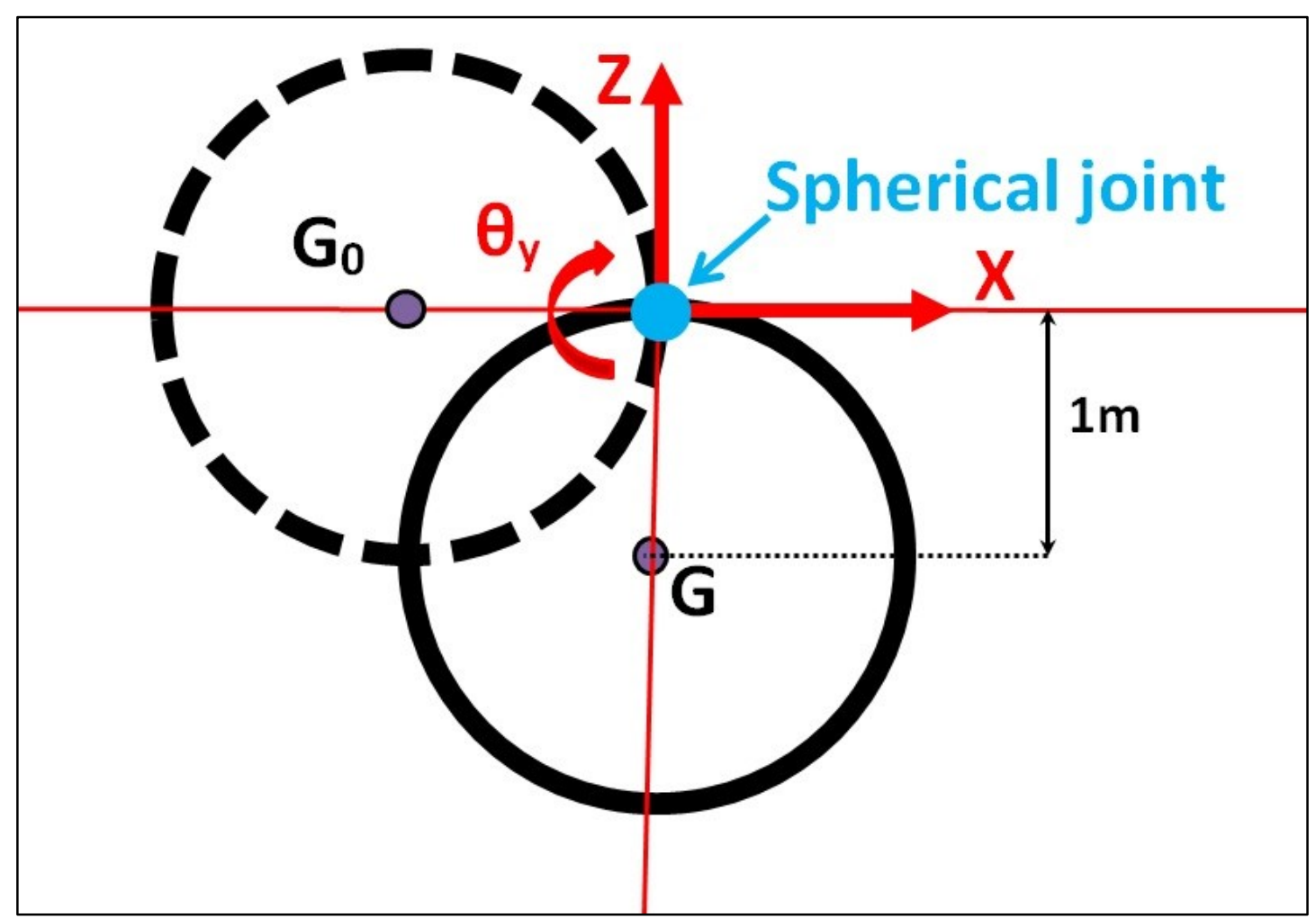

Figure 37: Rotating-ring layout. 


\subsection{Definition of body links}

The body links (kinematic constraints) are given by the following equations:

$$
\begin{aligned}
& \mathrm{f}_{1}\left(\mathrm{x}, \mathrm{z}, \theta_{\mathrm{y}}\right)=\mathrm{x}+\sin \left(\theta_{\mathrm{y}}\right)=0, \\
& \mathrm{f}_{2}\left(\mathrm{x}, \mathrm{z}, \theta_{\mathrm{y}}\right)=\mathrm{z}+\cos \left(\theta_{\mathrm{y}}\right)=0,
\end{aligned}
$$

being $\mathrm{x}, \mathrm{y}, \mathrm{z}$ the coordinates of the gravity center. The initial conditions are $\mathrm{x}^{0}=-1, \mathrm{z}^{0}=0$, and $\theta_{\mathrm{y}}^{0}=$ $90^{\circ}$. In a specific time step $t$ and iteration $k+1$, the constraint $\mathrm{f}_{\mathrm{i}}\left(\mathrm{x}^{\mathrm{t}, \mathrm{k}+1}, \mathrm{y}^{\mathrm{t}, \mathrm{k}+1}, \theta_{y}^{\mathrm{t}, \mathrm{k}+1}\right)=0$ is estimated via Eq. (4-10):

$$
\begin{aligned}
\mathrm{f}_{\mathrm{i}}\left(\mathrm{x}^{\mathrm{t}, \mathrm{k}}, \mathrm{y}^{\mathrm{t}, \mathrm{k}}, \mathrm{z}^{\mathrm{t}, \mathrm{k}}\right) & +\left(\frac{\partial f_{i}}{\partial x}\right)^{t, k}\left(\mathrm{x}^{\mathrm{t}, \mathrm{k}+1}-\mathrm{x}^{\mathrm{t}, \mathrm{k}}\right)+\left(\frac{\partial f_{i}}{\partial z}\right)^{t, k}\left(\mathrm{z}^{\mathrm{t}, \mathrm{k}+1}-\mathrm{z}^{\mathrm{t}, \mathrm{k}}\right) \\
+ & \left(\frac{\partial f_{i}}{\partial \theta_{\mathrm{y}}}\right)^{t, k}\left(\theta_{y}^{\mathrm{t}, \mathrm{k}+1}-\theta_{y}^{\mathrm{t}, \mathrm{k}}\right)=0
\end{aligned}
$$

where

$$
\begin{aligned}
& \left(\frac{\partial f_{1}}{\partial x}\right)^{t, k}=1 ;\left(\frac{\partial f_{1}}{\partial z}\right)^{t, k}=0 ;\left(\frac{\partial f_{1}}{\partial \theta_{\mathrm{y}}}\right)^{t, k}=\cos \left(\theta_{y}^{\mathrm{t}, \mathrm{k}}\right), \\
& \left(\frac{\partial f_{2}}{\partial x}\right)^{t, k}=0 ;\left(\frac{\partial f_{2}}{\partial z}\right)^{t, k}=1 ; \quad\left(\frac{\partial f_{2}}{\partial \theta_{\mathrm{y}}}\right)^{t, k}=-\sin \left(\theta_{y}^{\mathrm{t}, \mathrm{k}}\right) .
\end{aligned}
$$

Then, Eq. (7-2) can be written as:

$$
\begin{aligned}
& \mathrm{x}^{\mathrm{t}, \mathrm{k}+1}+\cos \left(\theta_{y}^{\mathrm{t}, \mathrm{k}}\right) \theta_{y}^{\mathrm{t}, \mathrm{k}+1}=-\sin \left(\theta_{\mathrm{y}}^{\mathrm{k}}\right)+\cos \left(\theta_{y}^{\mathrm{t}, \mathrm{k}}\right) \theta_{y}^{\mathrm{t}, \mathrm{k}}, \\
& \mathrm{z}^{\mathrm{t}, \mathrm{k}+1}-\sin \left(\theta_{y}^{\mathrm{t}, \mathrm{k}}\right) \theta_{y}^{\mathrm{t}, \mathrm{k}+1}=-\cos \left(\theta_{\mathrm{y}}^{\mathrm{k}}\right)-\sin \left(\theta_{y}^{\mathrm{t}, \mathrm{k}}\right) \theta_{y}^{\mathrm{t}, \mathrm{k}} .
\end{aligned}
$$

Let us remind the reader that $\mathrm{f}_{\mathrm{i}}\left(\mathrm{x}^{\mathrm{t}, \mathrm{k}}, \mathrm{z}^{\mathrm{t}, \mathrm{k}}, \theta_{y}^{\mathrm{t}, \mathrm{k}}\right)=0$ is not fulfilled in general during the iterative process, but it is fulfilled when the iterative process reaches convergence $\left|\mathbf{x}^{\mathrm{t}, \mathrm{k}+1}-\mathbf{x}^{\mathrm{t}, \mathrm{k}}\right|<\epsilon$.

By means of the Newmark's integration scheme used in the body dynamics solver, the acceleration and displacements are related (see Eq. (4-12)):

$$
\mathbf{x}^{t, k+1}=\mathbf{x}^{t-\Delta t}+\Delta t \dot{\mathbf{x}}^{t-\Delta t}+\frac{\Delta \mathrm{t}^{2}}{2}\left((1-2 \beta) \ddot{\mathbf{x}}^{t-\Delta t}+2 \beta \ddot{\mathbf{x}}^{t, k+1}\right),
$$

where $\beta$ is a parameter of the integration scheme. Inserting Eq. (7-7) into Eqs. (7-5)-(7-6):

$$
\begin{aligned}
& \ddot{\mathrm{x}}^{t, k+1}+\cos \left(\theta_{y}^{\mathrm{t}, \mathrm{k}}\right) \ddot{\theta}_{\mathrm{y}}^{t, k+1}=\mathrm{c}_{1}^{\mathrm{t}, \mathrm{k}}, \\
& \ddot{\mathrm{z}}^{t, k+1}-\sin \left(\theta_{y}^{\mathrm{t}, \mathrm{k}}\right) \ddot{\theta}_{\mathrm{y}}^{t, k+1}=\mathrm{c}_{2}^{\mathrm{t}, \mathrm{k}},
\end{aligned}
$$

where $c_{i}^{\mathrm{t}, \mathrm{k}}$ are terms that must be updated after each iteration, and: 


$$
\begin{aligned}
c_{1}^{t, k}=\frac{1}{\Delta \mathrm{t}^{2} \beta}( & \left.-\sin \left(\theta_{\mathrm{y}}^{\mathrm{k}}\right)+\cos \left(\theta_{y}^{\mathrm{t}, \mathrm{k}}\right) \theta_{y}^{\mathrm{t}, \mathrm{k}}\right) \\
& -\frac{1}{\Delta \mathrm{t}^{2} \beta}\left(\mathrm{x}^{t-\Delta t}+\Delta \mathrm{t} \dot{\mathrm{x}}^{t-\Delta t}+(1-2 \beta) \frac{\Delta \mathrm{t}^{2}}{2} \ddot{\mathrm{x}}^{t-\Delta t}\right) \\
& -\frac{\cos \left(\theta_{y}^{\mathrm{t}, \mathrm{k}}\right)}{\Delta \mathrm{t}^{2} \beta}\left(\theta_{\mathrm{y}}^{t-\Delta t}+\Delta \mathrm{t} \dot{\theta}_{\mathrm{y}}^{t-\Delta t}+(1-2 \beta) \frac{\Delta \mathrm{t}^{2}}{2} \ddot{\theta}_{y}^{t-\Delta t}\right), \\
c_{2}^{t, k}=\frac{1}{\Delta \mathrm{t}^{2} \beta}( & \left.-\cos \left(\theta_{\mathrm{y}}^{\mathrm{k}}\right)-\sin \left(\theta_{y}^{\mathrm{t}, \mathrm{k}}\right) \theta_{y}^{\mathrm{t}, \mathrm{k}}\right) \\
& -\frac{1}{\Delta \mathrm{t}^{2} \beta}\left(\mathrm{z}^{t-\Delta t}+\Delta \mathrm{tz} \dot{\mathrm{z}}^{t-\Delta t}+(1-2 \beta) \frac{\Delta \mathrm{t}^{2}}{2} \ddot{\mathrm{z}}^{t-\Delta t}\right) \\
& +\frac{\sin ^{t}\left(\theta_{y}^{\mathrm{t}, \mathrm{k}}\right)}{\Delta \mathrm{t}^{2} \beta}\left(\theta_{\mathrm{y}}^{t-\Delta t}+\Delta \mathrm{t} \dot{\theta}_{\mathrm{y}}^{t-\Delta t}+(1-2 \beta) \frac{\Delta \mathrm{t}^{2}}{2} \ddot{\theta}_{y}^{t-\Delta t}\right),
\end{aligned}
$$

or:

$$
\begin{aligned}
c_{1}^{t, k} & =-\frac{1}{\Delta \mathrm{t}^{2} \beta}\left(\mathrm{x}^{t, k}+\sin \left(\theta_{\mathrm{y}}^{\mathrm{k}}\right)\right)+\ddot{\mathrm{x}}^{t, k}+\cos \left(\theta_{y}^{\mathrm{t}, \mathrm{k}}\right) \ddot{\theta}_{y}^{t, k}, \\
\mathrm{c}_{2}^{t, k} & =-\frac{1}{\Delta \mathrm{t}^{2} \beta}\left(\mathrm{z}^{t, k}+\cos \left(\theta_{\mathrm{y}}^{\mathrm{k}}\right)\right)+\ddot{\mathrm{z}}^{t, k}-\sin \left(\theta_{y}^{\mathrm{t}, \mathrm{k}}\right) \ddot{\theta}_{y}^{t, k} .
\end{aligned}
$$

Lagrange multipliers are now introduced to include the constraints equations into the body dynamics (see Eq.(4-23)):

$$
\begin{gathered}
{\left[\begin{array}{ccccc}
M & 0 & 0 & 1 & 1 \\
0 & M & 0 & \cos \left(\theta_{y}^{\mathrm{t}, \mathrm{k}}\right) & -\sin \left(\theta_{y}^{\mathrm{t}, \mathrm{k}}\right) \\
0 & 0 & I & 0 & 0 \\
1 & \cos \left(\theta_{y}^{\mathrm{t}, \mathrm{k}}\right) & 0 & 0 & 0 \\
1 & -\sin \left(\theta_{y}^{\mathrm{t}, \mathrm{k}}\right) & 0 & 0 & 0
\end{array}\right]\left[\begin{array}{c}
(1-\alpha) \ddot{\mathrm{x}}^{t, k+1} \\
(1-\alpha) \ddot{\mathrm{z}}^{t, k+1} \\
(1-\alpha) \ddot{\theta}_{y}^{t, k+1} \\
\lambda^{t, k+1} \\
\mu^{t, k+1}
\end{array}\right]} \\
=\left[\begin{array}{c}
\mathrm{F}_{x}^{t, k} \\
\mathrm{~F}_{z}^{t, k} \\
\mathrm{M}_{y}^{t, k} \\
\mathrm{c}_{1}^{t, k} \\
\mathrm{c}_{2}^{t, k}
\end{array}\right]-\alpha\left[\begin{array}{c}
M \ddot{\mathrm{x}}^{t-\Delta t} \\
M \ddot{\mathrm{z}}^{t-\Delta t} \\
\ddot{\theta}_{y}^{t-\Delta t} \\
\mathrm{c}_{1}^{t, k} \\
\mathrm{c}_{2}^{t, k}
\end{array}\right],
\end{gathered}
$$

where $M$ is the ring mass, $I$ is the ring inertia, $\lambda$ and $\mu$ are the Lagrange multipliers, $\mathrm{F}_{\mathrm{x}}, \mathrm{F}_{\mathrm{y}}$ and $\mathrm{M}_{\mathrm{y}}$ are the external forces and moment acting on the ring, and $\alpha$ is the alpha parameter of the alpha BossakNewmark scheme (see section 4.5.2).

\subsection{Analysis of results}

A simulation has been carried out for 1000 seconds with a time step of $0.01 \mathrm{~s}$. An alpha parameter $\alpha=0$ provided unstable solution due to the introduction of energy into the system by the reaction 
forces. Hence, the alpha parameter had to be modified to $\alpha=-0.002$ to introduce some energy dissipation.

Figure 38 shows the surge, heave, and pitch movements of the ring for the time window between $990 \mathrm{~s}$ and 1000s. The expected oscillating motion is observed, with almost no dissipation on the motion amplitudes.

Figure 39 shows the residuals of the body links equations. Errors for both constraints are in the order of $10^{-6}$, while the terms of the constraint equation are in the order of 1 . Therefore, it can be stated that convergence was reached in order to fulfill the body links conditions, and the tolerance was small enough. Moreover, no sign of a steady increase with time is observed.

Figure 40 shows the evolution of the energy of the system over time. The initial energy is the potential energy at the initial position with zero velocity $\left(E_{0}=M g \Delta z=9.80665 \mathrm{~J}\right)$. After 1000 second of simulation, less than $1 \%$ of the initial energy has been lost due to numerical dissipation.

\subsection{Summary}

The body dynamics of a ring rotating harmonically around a spherical joint under the action of gravity has been simulated using the numerical techniques presented in Chapter 4 . Details on the derivation of the equations to be implemented have been provided. Results show that the dynamics has been solved properly, including the constraints imposed by the spherical joint. Also, low numerical dissipation has been introduced by the alpha Bossak-Newmark time integrator scheme.

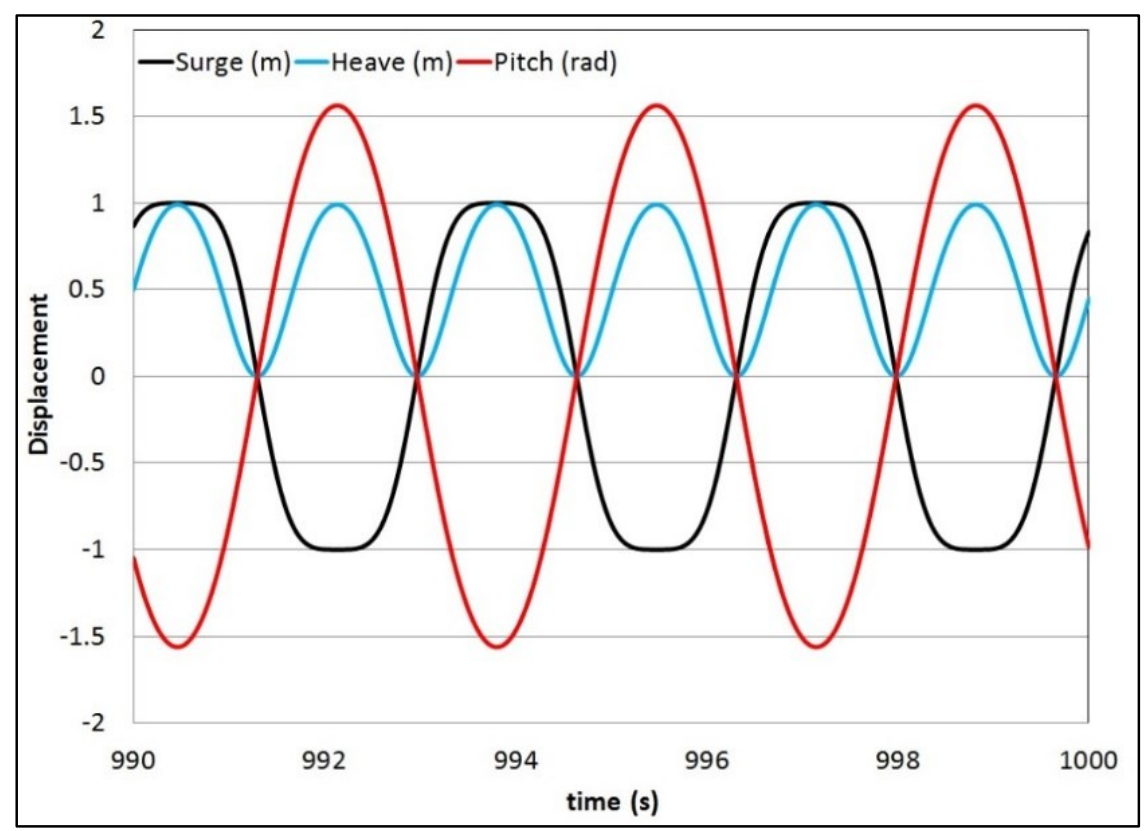

Figure 38: Rotating-ring movements. 


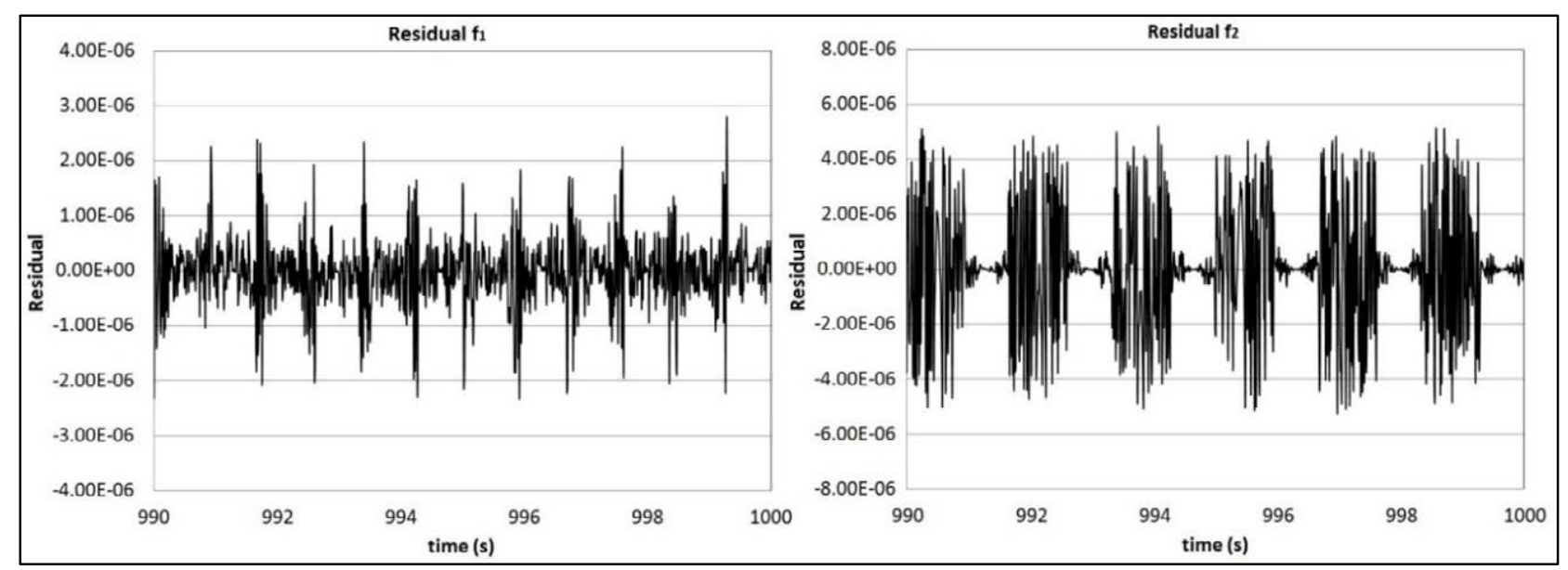

Figure 39: Body links residuals.

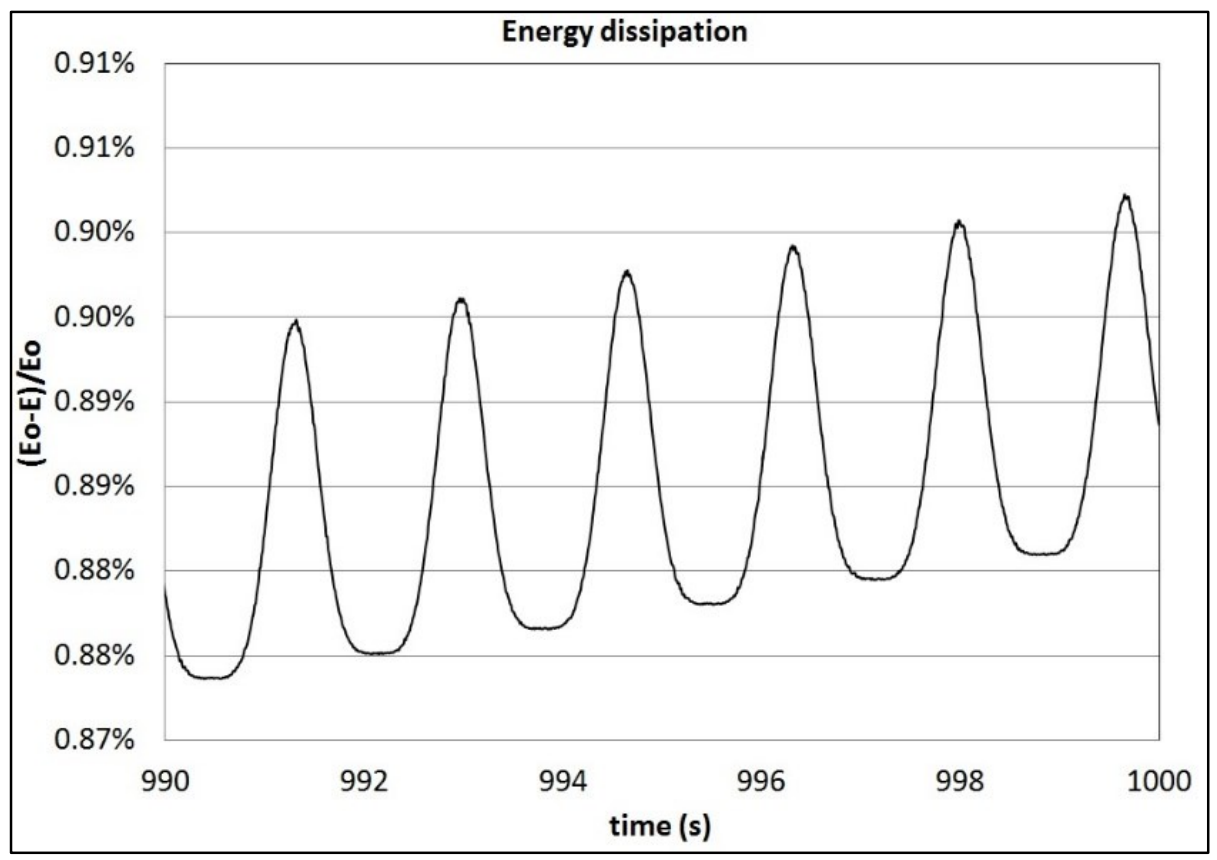

Figure 40: Energy dissipation along time. 


\section{Chapter 8. SOLVER ACCELERATION}

\subsection{Introduction}

Most of the computational effort of the proposed numerical algorithms is spent in solving the linear system resulting from the wave diffraction-radiation problem. Therefore, the main focus, in order to reduce the computational time, is speeding up this linear solver. Two techniques, that can be used combined, have been analyzed: the use of a deflated solver, and the use of parallel computing in graphic processing units.

\subsection{Solver deflation}

\subsubsection{Solver deflation: introduction}

Deflation works by considering piecewise constant approximations on coarse sub-domains of the computational domain. These piecewise constant approximations are associated to the low frequencies eigenmodes of the solution, which are also the slow convergence modes [54,55]. Then, these slow modes can be quickly approximated by solving a smaller linear system of equations that can be incorporated to the traditional preconditioned iterative solvers in order to accelerate the convergence of the solution. Hence, the use of preconditioning techniques is compatible with the use of deflation. Details on how deflation is implemented within the iterative solver can be found in [54].

The first step is to divide the domain into a set of coarse sub-domains capable of capturing the slow modes. Several techniques have been developed for this purpose [54,55]. Some authors have proposed a technique based on using seed-points to start building the sub-domains, and sub-domains are created by associating nodes to their closer seeds [54]. This technique has the disadvantage of requiring prescribing the seeds.

The algorithm developed in this work is based on level structure criteria, where nodes are associated to a central node based on the level structure rooted at this central node (concept from graph theory). A maximum level structure "L" is prescribed, where L refers to the neighboring level with respect to the central node. Figure 41 shows a domain decomposition obtained using this level structure technique with level $\mathrm{L}=2$. Ordered pair (sub-domain, level) is used to identify nodes and the subdomains they belong to, and their corresponding structure level. Notice that a node will accept to belong to a new sub-domain, if and only if, its structure level in that sub-domain is lower than its level 
in its current sub-domain. The algorithm used to obtain such decomposition is presented step by step next:

\section{Step 0: Assigned level L+1 to all nodes}

Step 1: Start building sub-domain 0: offer level 0 to the first node of the mesh (root node for sub-domain 0). It will become node (sub-domain, level) $=(0,0)$

Step 2: Identify neighbors of the root node (node $(0,0))$ and offer them level 1: nodes $(0,1)$

Step 3: Identify neighbors of nodes $(0,1)$, and offer them level 2 : nodes $(0,2)$

Step 4: The procedure is repeated until the prescribed level " $\mathrm{L}$ " is reached

Step 5: The first node still with level $\mathrm{L}+1$ (based on the numbering of the nodes) is used as root node for sub-domain 1 , and it is given level 0 , becoming the root node $(1,0)$

Step 6: Identify neighbors of the root node $(1,0)$ and offer them level 1 : nodes $(1,1)$ (Notice that some $(0, \mathrm{~L})$ nodes might become $(1,1))$

Step 7: Identify neighbors of nodes $(1,1)$, and offer them level 2: nodes $(1,2)$. (Notice that some $(0, \mathrm{~L})$ or $(0, \mathrm{~L}-1)$ nodes might become $(1,2)$ if $\mathrm{L}>2$ or $\mathrm{L}-1>2$ respectively)

Step 8: The procedure is repeated until the nodes $(1, \mathrm{~L})$ are identified

Step 9: Repeat Steps 5-8 until there is no node with level $\mathrm{L}+1$

This algorithm guarantees that any node cannot have a lower structure level than any other root node. It also guarantees the higher the prescribed level, the lower the number of sub-domains created.

Although the previous algorithm provides good results, it can be improved by using it twice. In a first round, rather than using the prescribed level L, a structure level $2 \mathrm{~L}$ is used instead. Then a first decomposition is found with a lower number of sub-domains and twice the maximum structure level. In a second round, the procedure is repeated with a maximum structure level $\mathrm{L}$ and using as root those nodes with higher levels. Figure 41 and Figure 42 show the sub-domain decomposition obtained using the one two rounds technique respectively. The first one shows the decomposition after the first round at level 2L, and the second shows the decomposition after the second round at level L starting from the first decomposition at level 2L. Notice that the level at the sub-domains boundaries is lower or equal than the prescribed level L, and always minimum with respect to any central node (node of level zero).

The recommended number of sub-domains might depend on the specific case under study. Usually in the order of hundred is a recommended value. If the matrix of the linear system to be solved remains the same during the calculation, the sub-domain decompositions will have to be carried out just once at the beginning. 
The deflated system has to be solved every iteration of the solver. In case the equations to be solved remain the same along the simulation, so does the deflated matrix. The inverse of the deflated matrix can be calculated just in the first time step, and then be stored. Hence, the resolution of the deflated system within each iteration can be substituted by a matrix vector multiplication.

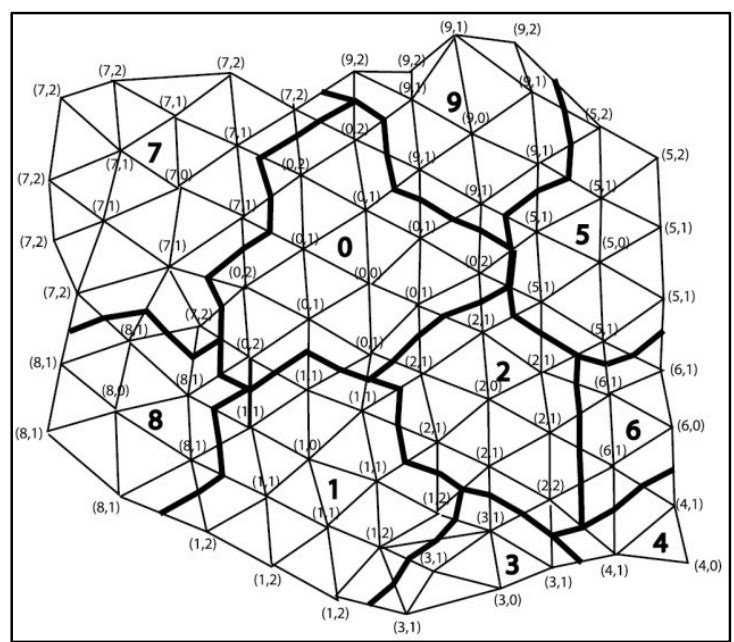

Figure 41: Sub-domain decomposition using the neighboring level algorithm.

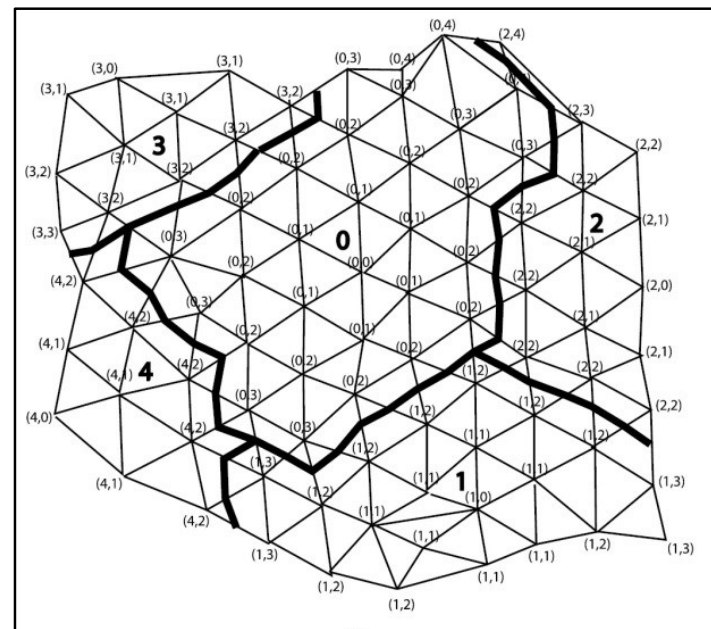

(a)

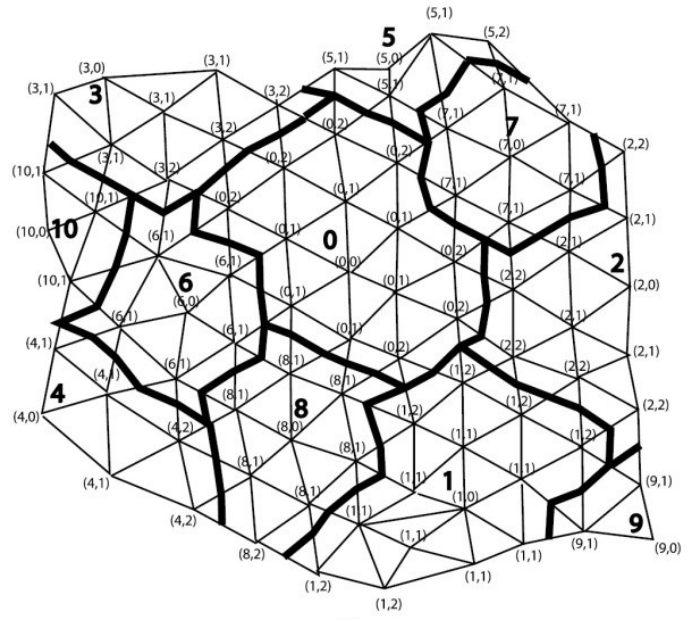

(b)

Figure 42: Sub-domain decomposition using the two-rounds neighboring level algorithm. (a) Decomposition after first round. (b) Decomposition after second round.

\subsubsection{Solver deflation: demonstration}

In order to check the performance of deflated preconditioned conjugate gradient in our problems, simulations using the ISSC TLP platform (analyzed in section 5.5) have been carried out using five different meshes. For each case, different levels of deflation have been used, and they have been compared to a non-deflated case. Table 12 provides the particulars of each case. 
Table 12: Case studies particulars

\begin{tabular}{|c|c|c|c|c|c|}
\hline & Case 1 & Case 2 & Case 3 & Case 4 & Case 5 \\
\hline Number of Nodes & 17804 & 51333 & 153758 & 459538 & 913149 \\
\hline Number of Elements & 101572 & 292705 & 792372 & 2335374 & 4640638 \\
\hline h (m) & 4 & 2 & 0.5 & 0.35 & 0.25 \\
\hline$\Delta \mathbf{t}(\mathbf{s})$ & 0.75 & 0.5 & 0.1 & 0.075 & 0.05 \\
\hline
\end{tabular}

Solver deflation is compatible with the use of preconditioners. Therefore, comparisons are made using the deflated conjugate gradient along with an ILU preconditioner. In the case of the deflated solver, different structure levels have been used, resulting in different number of sub-domains. In all cases, the same computer and CPU have been used.

Figure 43 shows the reduction in iterations for each case as the number of sub-domains changes. Table 13-Table 17 show the average number of iterations and speed up respect to the non-deflated case. The speed up has been obtained as the ratio of CPU times taken by the linear solver in the nondeflated and deflated cases. It can be observed that the number of iterations decreases as the dimension of the deflated subspace increases. This reduction in the number of iteration when using an ILU preconditioner indicates that the proposed technique for building sub-domains is performing well.

However, when using deflated solvers, a few operations are added in every solver iteration. Hence reducing iterations is not synonym of reducing CPU time. Based on the results obtained, it can be said that the larger the system, the larger the speed up that can be obtained, and the number of sub-domains must be larger as well. However, care must be taken because a wrong dimension of the deflated subspace might end up in increasing the CPU time. Moreover, for smaller cases, deflation might even not be capable of speeding up at all.

Deflation has been proved to be quite effective in reducing the number of iterations, which means that the sub-domain decomposition is working well. However, in our case study, deflation is not so effective in reducing CPU time. Moreover, depending on the level structure used, CPU time can be even degraded respect to the non-deflated solver. Therefore, choosing the right level structure is mandatory to optimize the performance of the deflation algorithm.

\subsection{GPU-CUDA acceleration}

\subsubsection{GPU-CUDA acceleration: introduction}

The fast development of the videogame industry is leading to higher computational capabilities of graphic processing units (GPUs). Hence, their use for heavy computations in computational fluid dynamics is becoming more common (see $[56,57]$ ). 


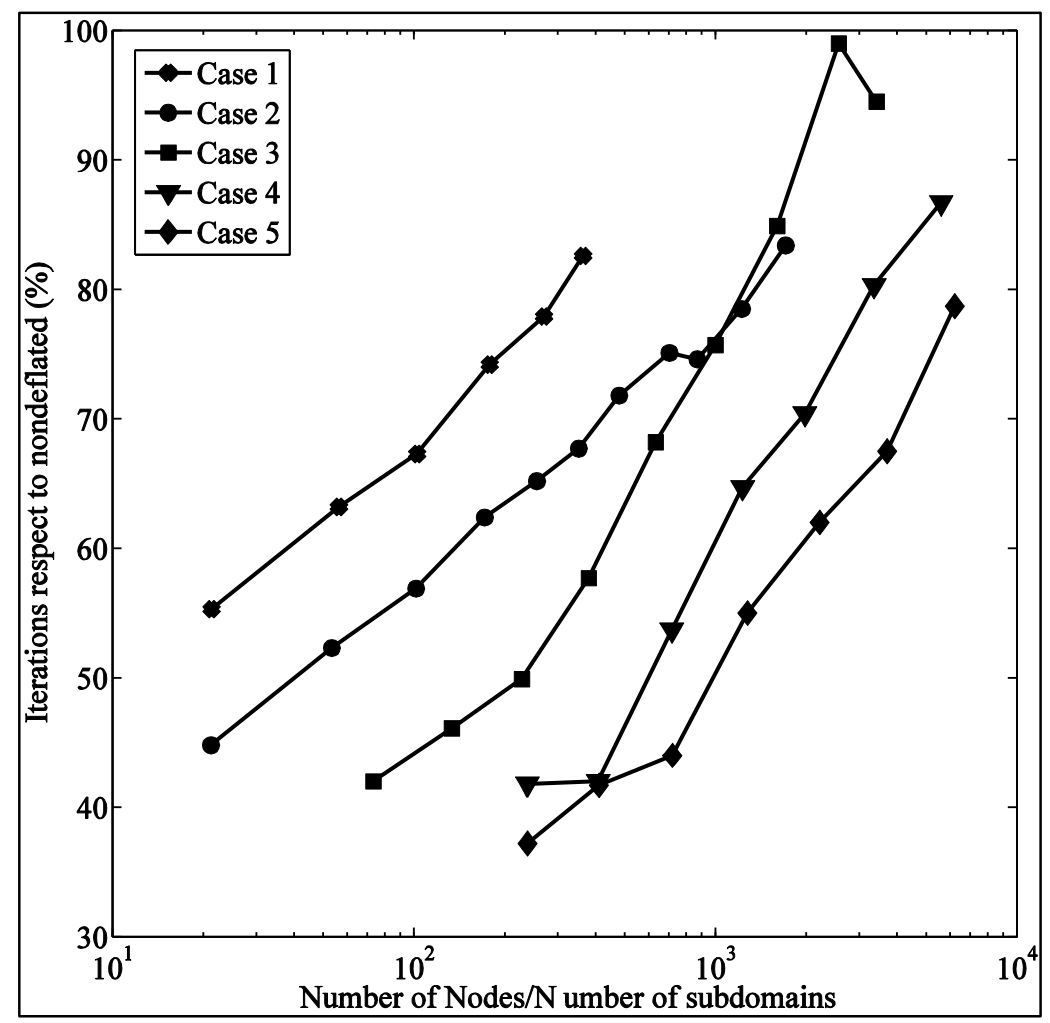

Figure 43: Deflated solver: number of iterations.

Table 13: Comparative among deflated solvers and non-deflated

Case 1: Number of nodes $=\mathbf{1 7 8 0 4}$

\begin{tabular}{|c|c|c|c|}
\hline Structure level & Number of Sub-domains & Average Number Iterations & Speed up \\
\hline $\mathbf{2}$ & $\mathbf{8 3 6}$ & 15.89 & $\mathrm{x} 0.556$ \\
\hline $\mathbf{3}$ & $\mathbf{3 1 6}$ & 18.18 & $\mathrm{x} 0.963$ \\
\hline $\mathbf{4}$ & $\mathbf{1 7 4}$ & 19.36 & $\mathrm{x} 0.989$ \\
\hline $\mathbf{5}$ & $\mathbf{1 0 0}$ & 21.34 & $\mathrm{x} 0.894$ \\
\hline $\mathbf{6}$ & $\mathbf{6 6}$ & 22.39 & $\mathrm{x} 0.871$ \\
\hline $\mathbf{7}$ & $\mathbf{4 9}$ & 23.74 & $\mathrm{x} 0.829$ \\
\hline \multicolumn{4}{|c|}{ Non-deflated } \\
\hline
\end{tabular}

Table 14: Comparative among deflated solvers and non-deflated

\begin{tabular}{|c|c|c|c|}
\hline \multicolumn{4}{|c|}{ Case 2: Number of nodes $=51333$} \\
\hline Structure level & Number of Sub-domains & Average Number Iterations & Speed up \\
\hline 2 & 2419 & 17.34 & $\mathrm{x} 0.310$ \\
\hline 3 & 963 & 20.23 & $\mathrm{x} 0.908$ \\
\hline 4 & 506 & 22.00 & x1.081 \\
\hline 5 & 299 & 24.15 & $\mathrm{x} 1.055$ \\
\hline 6 & 201 & 25.20 & $\mathrm{x} 1.023$ \\
\hline 7 & 146 & 26.17 & $\mathrm{x} 0.943$ \\
\hline 8 & 107 & 27.77 & $\mathrm{x} 0.907$ \\
\hline 9 & 73 & 29.04 & $\mathrm{x} 0.889$ \\
\hline 10 & 59 & 28.84 & $\mathrm{x} 0.912$ \\
\hline 11 & 42 & 30.36 & $\mathrm{x} 0.863$ \\
\hline 12 & 30 & 32.27 & $\mathrm{x} 0.789$ \\
\hline \multicolumn{2}{|c|}{ Non-deflated } & 38.68 & $\mathrm{x} 1$ \\
\hline
\end{tabular}


Table 15: Comparative among deflated solvers and non-deflated

\begin{tabular}{|c|c|c|c|}
\hline \multicolumn{4}{|c|}{ Case 3: Number of nodes $=153758$} \\
\hline Structure level & Number of Sub-domains & Average Number Iterations & Speed up \\
\hline 4 & 2101 & 20.82 & $\mathrm{x} 0.77$ \\
\hline 5 & 1153 & 22.82 & $\mathrm{x} 1.20$ \\
\hline 6 & 675 & 24.71 & $\mathrm{x} 1.30$ \\
\hline 7 & 405 & 28.59 & $\mathrm{x} 1.17$ \\
\hline 8 & 243 & 33.8 & $\mathrm{x} 1.00$ \\
\hline 9 & 154 & 37.5 & $\mathrm{x} 0.91$ \\
\hline 10 & 96 & 42.06 & $\mathrm{x} 0.82$ \\
\hline 11 & 60 & 49.03 & $\mathrm{x} 0.71$ \\
\hline 12 & 45 & 46.83 & $\mathrm{x} 0.72$ \\
\hline \multicolumn{2}{|c|}{ Non-deflated } & 49.53 & $\mathrm{x} 1$ \\
\hline
\end{tabular}

Table 16: Comparative among deflated solvers and non-deflated

\begin{tabular}{|c|c|c|c|}
\hline \multicolumn{5}{|c|}{ Case 4: Number of nodes $=\mathbf{4 5 9 5 3 8}$} \\
\hline Structure level & Number of Sub-domains & Average Number Iterations & Speed up \\
\hline $\mathbf{6}$ & $\mathbf{1 9 3 6}$ & 25.46 & $\mathrm{x} 1.01$ \\
\hline $\mathbf{7}$ & $\mathbf{1 1 2 8}$ & 25.58 & $\mathrm{x} 1.39$ \\
\hline $\mathbf{8}$ & $\mathbf{6 4 1}$ & 32.66 & $\mathrm{x} 1.22$ \\
\hline $\mathbf{9}$ & $\mathbf{3 7 4}$ & 39.38 & $\mathrm{x} 1.03$ \\
\hline $\mathbf{1 0}$ & $\mathbf{2 3 2}$ & 42.86 & $\mathrm{x} 0.94$ \\
\hline $\mathbf{1 1}$ & $\mathbf{1 3 7}$ & 48.90 & $\mathrm{x} 0.84$ \\
\hline $\mathbf{1 2}$ & $\mathbf{8 2}$ & 52.76 & $\mathrm{x} 0.76$ \\
\hline \multicolumn{4}{|c|}{ Non-deflated } \\
\hline
\end{tabular}

Table 17: Comparative among deflated solvers and non-deflated

\begin{tabular}{|c|c|c|c|}
\hline \multicolumn{5}{|c|}{ Case 5: Number of nodes $=\mathbf{9 1 3 1 4 9}$} \\
\hline Structure level & Number of Sub-domains & Average Number Iterations & Speed up \\
\hline $\mathbf{6}$ & $\mathbf{3 8 3 7}$ & 26.70 & $\mathrm{x} 0.54$ \\
\hline $\mathbf{7}$ & $\mathbf{2 2 2 9}$ & 29.95 & $\mathrm{x} 1.10$ \\
\hline $\mathbf{8}$ & $\mathbf{1 2 7 1}$ & 31.60 & $\mathrm{x} 1.40$ \\
\hline $\mathbf{9}$ & $\mathbf{7 1 7}$ & 39.55 & $\mathrm{x} 1.20$ \\
\hline $\mathbf{1 0}$ & $\mathbf{4 1 3}$ & 44.55 & $\mathrm{x} 1.07$ \\
\hline $\mathbf{1 1}$ & $\mathbf{2 4 6}$ & 48.50 & $\mathrm{x} 0.99$ \\
\hline $\mathbf{1 2}$ & $\mathbf{1 4 7}$ & 56.55 & $\mathrm{x} 0.83$ \\
\hline \multicolumn{4}{|}{ Non-deflated } \\
\hline
\end{tabular}

The implementation in this work is based on the well-known CUDA, a parallel computing platform and programming model invented by NVIDIA. It is focused in speeding up the iterative solver using the functions provided by the CUBLAS and CUSPARSE libraries.

Deflated and non-deflated preconditioned conjugate gradient (CG) algorithm has been implemented in CUDA, along with a sparse approximate inverse (SPAI) preconditioner [58] and incomplete LU decomposition (ILU) preconditioner. However, using the sparse lower and upper triangular solvers provided in the CUSPARSE library for ILU preconditioning resulted in poor 
performance. This poor performance can be expected since solving triangular system is not suitable for massive parallelization across tens of thousands threads [58], as required by GPUs in order to hide memory latency. Therefore we will omit the use of the ILU preconditioner when reporting GPU results.

Since the system matrix remains the same along the computation, preconditioners are calculated just once, in the first time step. Then, computational time invested in calculating preconditioners is negligible when compared to the total time spent in the linear solver during the whole simulation.

\subsubsection{GPU-CUDA acceleration: demonstration}

In this section an array of sixteen freely floating bodies in the presence of a regular wave is simulated. The case study consists of simulating the dynamics of an array of sixteen freely floating cylinders in the presence of a regular wave. Each cylinder is one meter in radius, and half meter in draft. Each one is freely floating without restriction. Then, since they are freely floating, the mass of each cylinder must equal the mass of the displaced water. The radii of gyration with respect to their own center of gravity are equal to one. The incident wave has a wave period of two seconds, amplitude of ten centimeters, and an incident direction of $22.5^{\circ}$. The cylinders are placed on a regular pattern $(4 \times 4)$ and the distance between the centers of adjacent cylinders is three meters. A fifty second simulations is carried out, and the time step used in each case has been calculated such as the dimensionless grid number $g \Delta t^{2} / h=0.5$, where $h$ represents the characteristic element size.

The case study is simulated with three levels of grid refinement (see Figure 44). Computational time spent to solve the linear system of equations in every time step during the simulations were measured and are reported next. The solver used is a non-deflated preconditioned conjugate gradient. Table 18 provides the particulars of the meshes and numerical parameters used. Figure 45 shows the free surface elevation at the end of the simulation.

Figure 46 compares the movements of the cylinder located in the upper right corner for the three meshes used. While some difference is found for the coarser mesh, results are very close for cases 2 and 3 as expected.

Table 18: Mesh properties and case study numerical parameters

\begin{tabular}{|l|c|c|c|c|}
\hline & Number of nodes & Number of elements & Characteristic element size (m) & Time step (s) \\
\hline Case 1 & 137556 & 799665 & 0.2 & 0.1010 \\
\hline Case 2 & 369410 & 2152774 & 0.1 & 0.0714 \\
\hline Case 3 & 1181302 & 6878083 & 0.05 & 0.0505 \\
\hline
\end{tabular}

Speed-up analyses are carried out when using different types of GPU/CPU architecture, as well as different types of preconditioners. When computing in parallel in the GPU, the sparse approximate inverse (SPAI) preconditioner will be used. When carrying out serial computation in the CPU, the SPAI and ILU preconditioners will be used. In this case study, the matrix system does not change 
along the simulation. Therefore, the preconditioner is only requested to be computed once. Also, let's remind that the use of the ILU preconditioner in GPU architectures is not recommended due to its poor parallelization across thousands of threads, as required by GPUs to hide memory latency.

In order to compare computational performance, we carried out simulations in four different platforms: two GPU platforms, and two CPU platforms. Table 19 provides the particulars of each platform used.

Table 19: Computing Plattforms used.

\begin{tabular}{|c|c|}
\hline Platform & Description \\
\hline GPU1 & NVIDIA GTX 280 \\
\hline CPU1 & Intel(R ) Core(TM )2 CPU Q9400 @ 2.66GHz 2.67GHz \\
\hline GPU2 & NVIDIA TESLA C2075 \\
\hline CPU2 & Intel(R ) Core(TM ) i7-3930K CPU @ 3.20GHz 3.20GHz \\
\hline
\end{tabular}

Since hardware evolve quite fast, it is necessary to take into account whether comparisons are made across hardware of the same generation. We have named GPU1 and CPU1 to the older platforms, belonging more or less to the same generation, and named CPU2 and GPU2 to the newer platforms, also belonging to the same generation. Therefore, when comparing computational time, it must be taken into account that the newer platforms were bought in 2012, while the older ones were bought in 2009.

Table 20 shows the computational time required for simulating fifty seconds with three different meshes and under different solver strategies. Notice that more than eighty percent of the computational time is spent in the linear solver. Therefore, speeding up this part of the code is the key point for acceleration. Table 21 and Table 22 show the speed up obtained as the ratio of time spent in the linear solver when using same generation CPU and GPU platforms.

When comparing GPU1 versus CPU1, the minimum speed up obtained in the linear solver are $\mathrm{x} 5.09, \mathrm{x} 6.73$, and $\mathrm{x} 8.83$ for cases 1,2 and 3 respectively. On the other hand, when comparing GPU2 versus CPU2, the minimum speed up obtained in the linear solver are $\mathrm{x} 3.98, \mathrm{x} 4.90$, and $\mathrm{x} 6.35$ for cases 1, 2 and 3 respectively. As expected, the speed up increases as the size of the case study increases.

When comparing GPU1 versus GPU2, it can be observed that the speed up obtained using the newer generation is in the order of 2 . We should also point out that the price of the newer is in the order of ten times the older.

Comparing the performance of GPU1 respect to CPU2, we observe that GPU1 performs better. It gets speed-ups of $x 2, x 2.58$, and $\times 3.53$ in each case study.

The fifty second simulation can be carried out with enough accuracy in some 20 minutes using GPU2. Since the length scale of the model is one meter, those fifty seconds are equivalent to 500 seconds if the radius of each cylinder were one hundred meters. This leads to a ratio between computational time and real time around 2 for some offshore engineering problems. 


\subsection{OpenMP parallelization}

OpenMP [59] has been used for parallelization of CPU solvers as well as for evaluating analytical formulas. For instance, when evaluating irregular seas modelled by spectra discretization based on Airy waves, a large number of waves might be used, requiring a significant CPU time compared to the solver CPU time. Open MP has been used in this work to reduce the CPU time of this type of computations.

\subsection{Summary}

Deflation has been proved to be quite effective in reducing the number of iterations required by the conjugate gradient. However, in our case study, deflation is not so effective in reducing CPU time as it is in reducing solver iterations. Solver deflation can speed up the solution and, the larger the size of the problem, the larger the speed up. But, depending on the level structure used, CPU time can be even degraded respect to the non-deflated solver. Therefore, choosing the right level structure based on the size of the problem is mandatory to optimize the performance of the deflated conjugate gradient.

GPUs have been used for solver acceleration. For this purpose, deflated and non-deflated preconditioned conjugate gradient (CG) algorithm has been implemented in CUDA, along with a sparse approximate inverse (SPAI) preconditioner and incomplete LU decomposition (ILU) preconditioner. Results indicate that GPUs-based solvers, using SPAI preconditioners, can perform faster than CPUs, and the speed-ups obtained in this work are in the range of 4-9, depending on the size of the problem, and the generation of GPU/CPU used. 


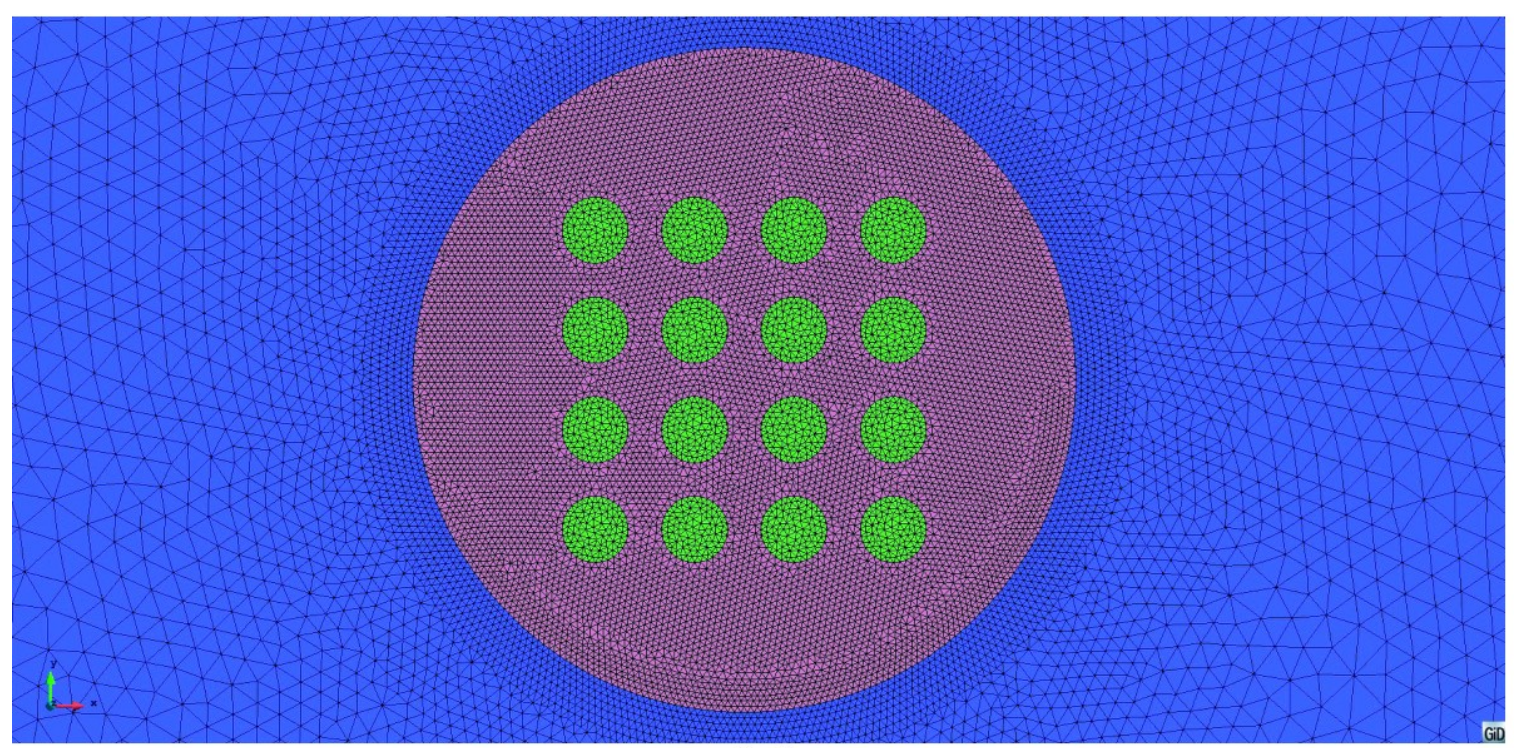

(a)

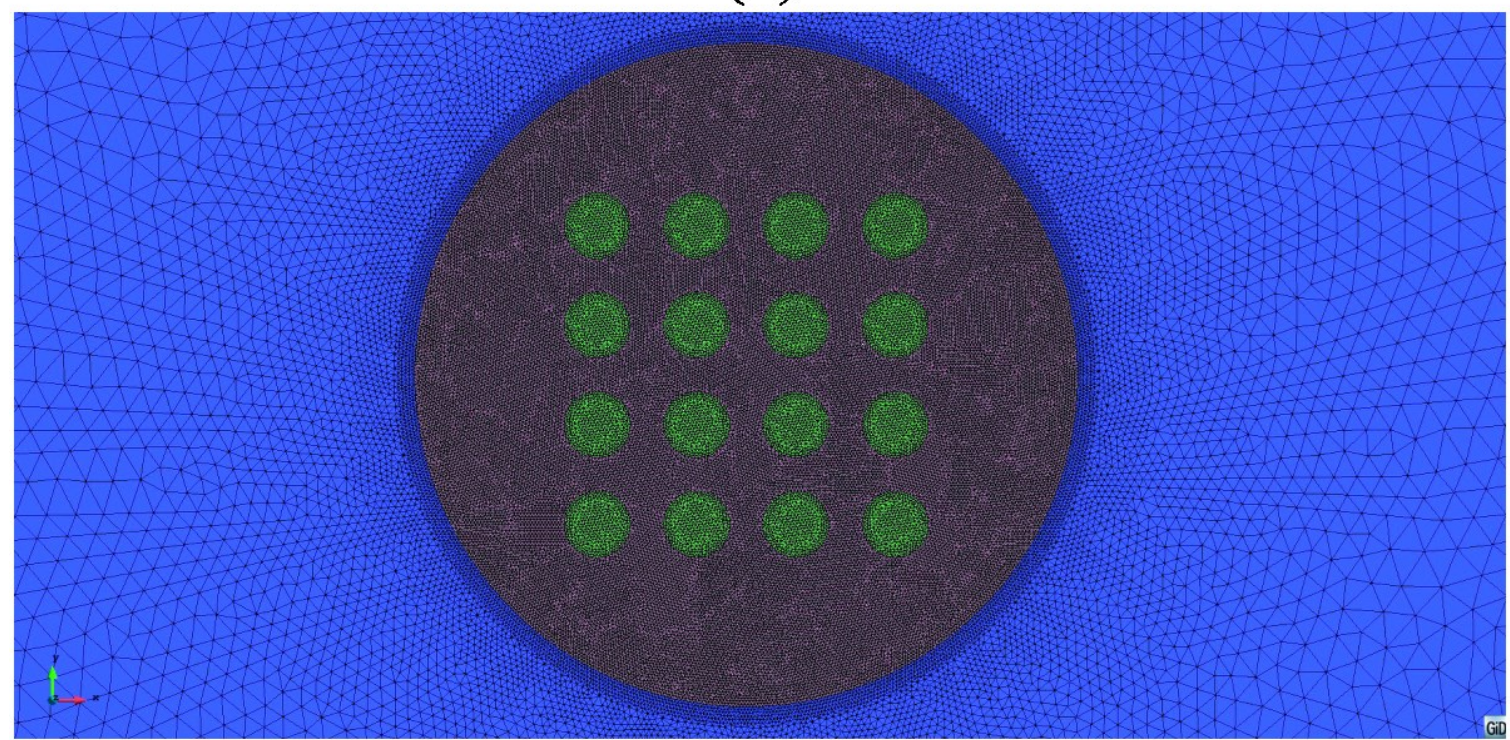

(b)

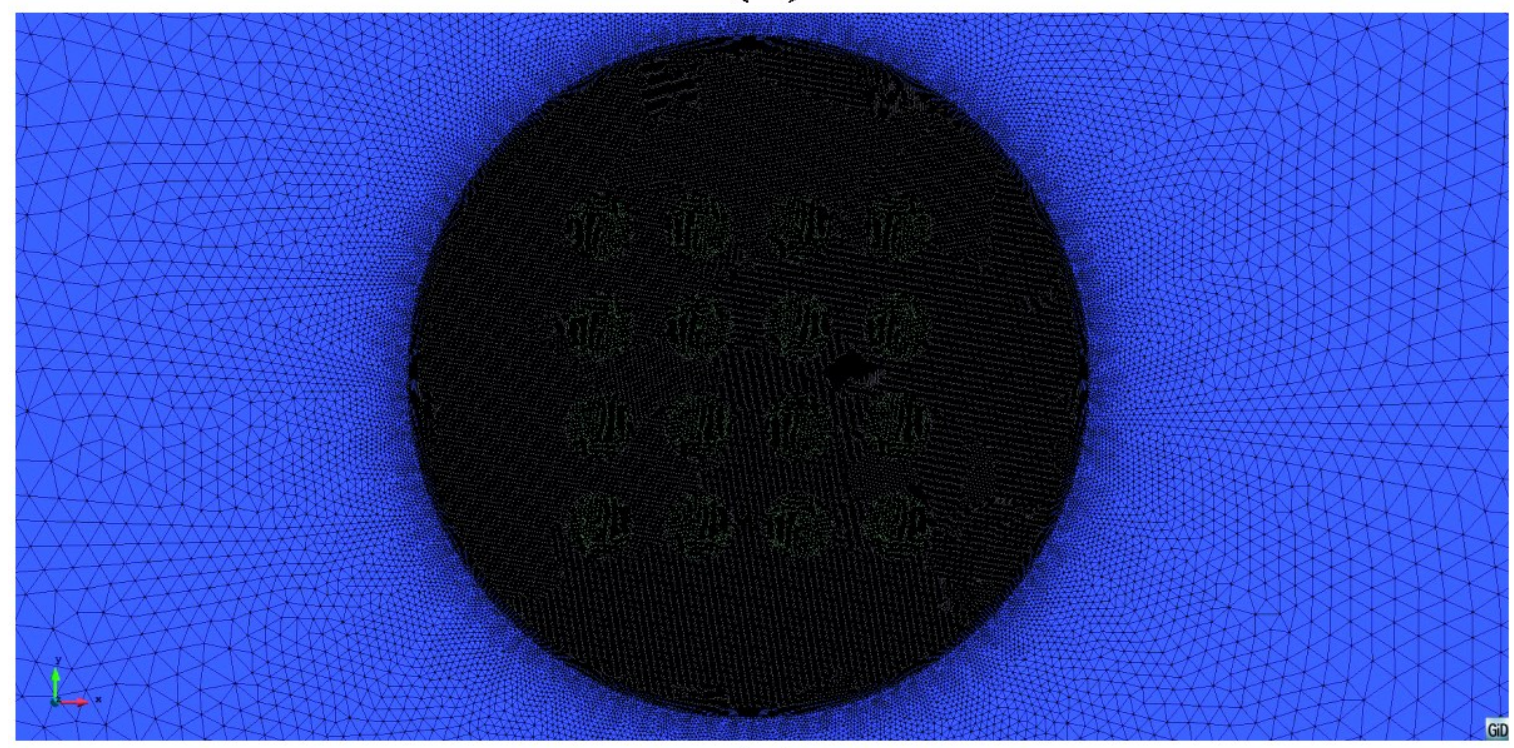

(c)

Figure 44: Mesh refinement for multibody system. 


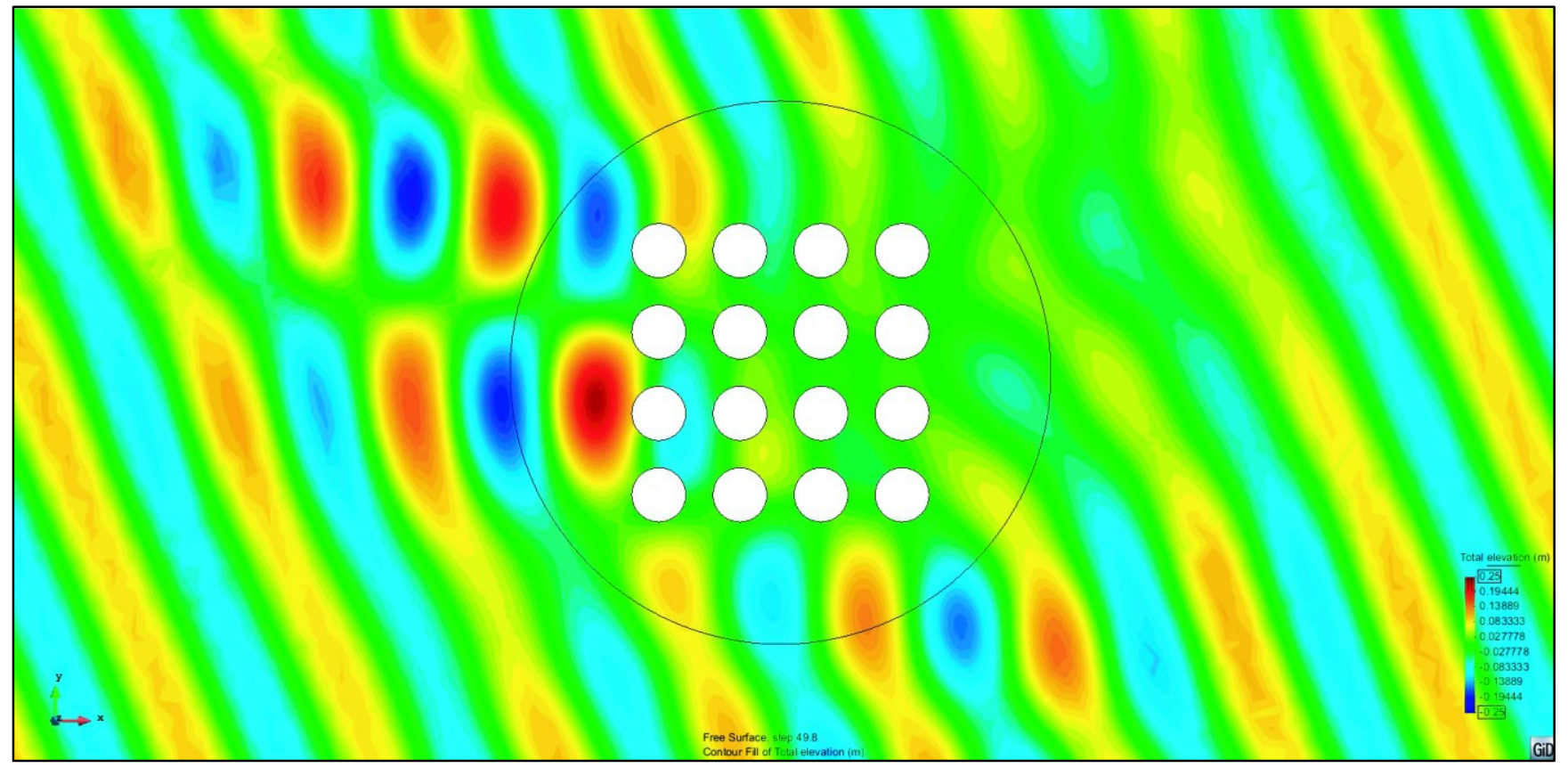

Figure 45: free surface contour field for multibody system and monochromatic incident wave. 

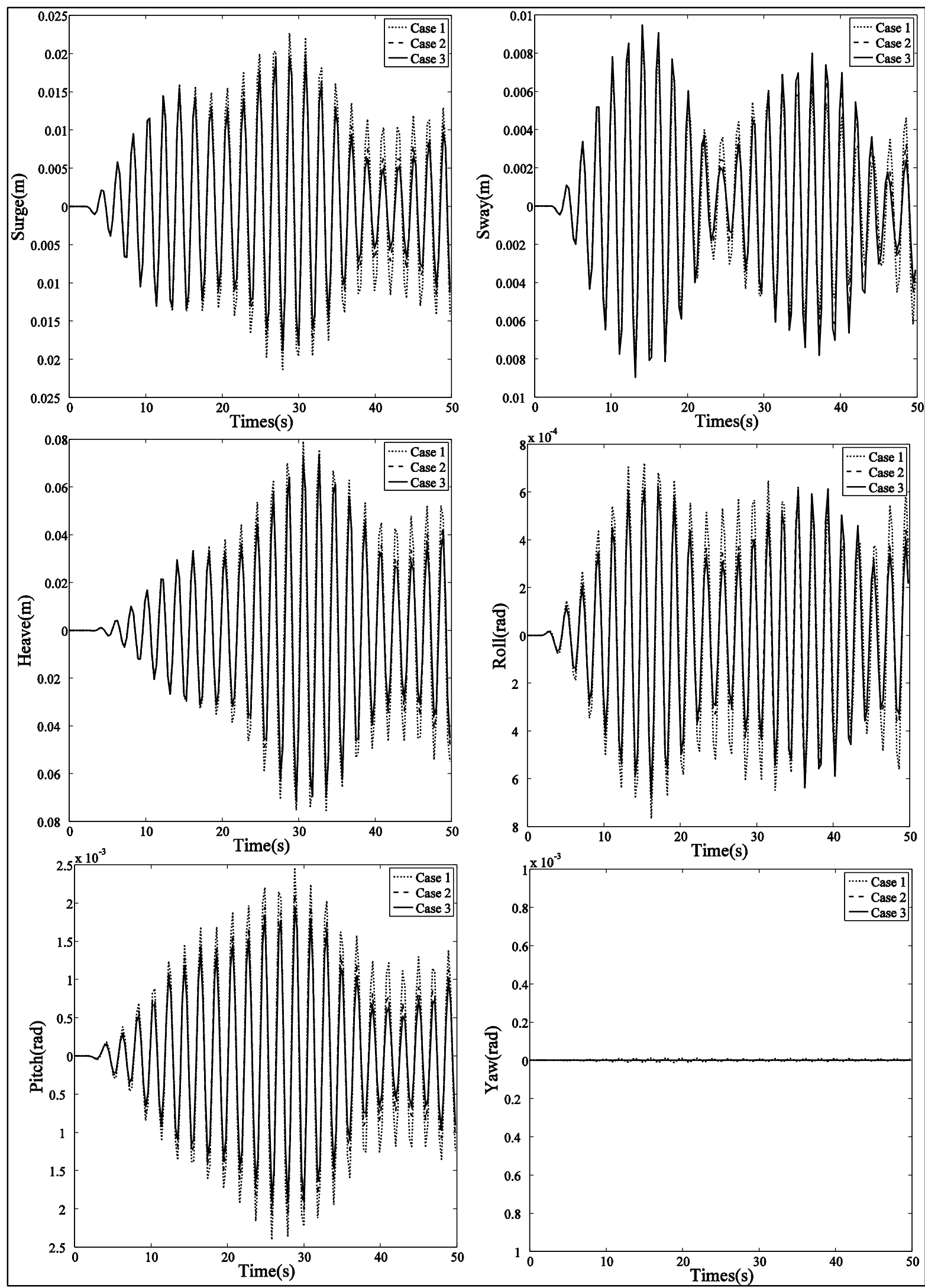

Figure 46:Multi-body simulation: movements of the body located at the upper right corner. Comparison using different level of mesh refinement. 
Table 20: Computational time spent for a fifty second simulation of an array sixteen floating cylinders.

\begin{tabular}{|c|c|c|c|c|c|}
\hline & PLATFORM & PRECONDITIONER & Solver time (s) & Total time (s) & Solver time(\%) \\
\hline \multirow{6}{*}{ Case 1} & GPU1 & \multirow{2}{*}{ SPAI } & 564 & 695 & 81.2 \\
\hline & \multirow{2}{*}{ CPU1 } & & 3256 & 3325 & 97.9 \\
\hline & & ILU & 2868 & 2938 & 97.6 \\
\hline & GPU2 & \multirow{2}{*}{ SPAI } & 282 & 314 & 89.7 \\
\hline & \multirow{2}{*}{ CPU2 } & & 1217 & 1249 & 97.5 \\
\hline & & ILU & 1123 & 1154 & 97.3 \\
\hline \multirow{6}{*}{ Case 2} & GPU1 & \multirow{2}{*}{ SPAI } & 2012 & 2284 & 88.1 \\
\hline & \multirow{2}{*}{ CPU1 } & & 15051 & 15312 & 98.3 \\
\hline & & ILU & 13549 & 13812 & 98.1 \\
\hline & GPU2 & CDAI & 1060 & 1178 & 90.0 \\
\hline & CDU? & 1 & 6052 & 6165 & 98.2 \\
\hline & C & ILU & 5198 & 5311 & 97.9 \\
\hline \multirow{6}{*}{ Case 3} & GPU1 & \multirow{2}{*}{ SPAI } & 8519 & 9854 & 86.4 \\
\hline & \multirow{2}{*}{ CPU1 } & & 85605 & 86740 & 98.7 \\
\hline & & ILU & 75223 & 76307 & 98.6 \\
\hline & GPU2 & \multirow{2}{*}{ SPAI } & 4743 & 5302 & 89.4 \\
\hline & \multirow{2}{*}{ CPU2 } & & 37479 & 37941 & 98.8 \\
\hline & & ILU & 30110 & 30588 & 98.4 \\
\hline
\end{tabular}

Table 21: Speed up obtained by using GPU1 respect to serial execution with CPU1.

\begin{tabular}{|c|c|c|c|c|c|c|}
\hline \multirow{2}{*}{} & \multicolumn{6}{|c|}{ Reference case } \\
\cline { 2 - 7 } & \multicolumn{3}{|c|}{ CPU1+SPAI } & \multicolumn{3}{c|}{ CPU1+ILU } \\
\cline { 2 - 7 } & Case 1 & Case 2 & Case 3 & Case 1 & Case 2 & Case 3 \\
\hline $\begin{array}{c}\text { GPU1+SPAI } \\
\text { (Speed-up) }\end{array}$ & $\mathrm{x} 5.77$ & $\mathrm{x} 7.48$ & $\mathrm{x} 10.04$ & $\mathrm{x} 5.09$ & $\mathrm{x} 6.73$ & $\mathrm{x} 8.83$ \\
\hline
\end{tabular}

Table 22: Speed up obtained by using GPU2 respect to serial execution with CPU2.

\begin{tabular}{|c|c|c|c|c|c|c|}
\hline \multirow{2}{*}{} & \multicolumn{6}{|c|}{ Reference case } \\
\cline { 2 - 7 } & \multicolumn{3}{|c|}{ CPU2+SPAI } & \multicolumn{3}{c|}{ CPU2+ILU } \\
\cline { 2 - 7 } & Case 1 & Case 2 & Case 3 & Case 1 & Case 2 & Case 3 \\
\hline $\begin{array}{c}\text { GPU2+SPAI } \\
\text { (Speed-up) }\end{array}$ & $\times 5.32$ & $\times 5.71$ & $\times 7.90$ & $\times 3.98$ & $\times 4.90$ & $\times 6.35$ \\
\hline
\end{tabular}


PART III: APLICATIONS 


\section{Chapter 9. ANALYSIS OF A SURFACE EFFECT SHIP SEAL DYNAMICS}

\subsection{Introduction}

A SES is a non-amphibious vehicle supported by an air cushion, with flexible seals at the bow and stern, and twin hulls, like a catamaran, at the sides. Due to the lack of air leakage at the craft sides, lift power can be reduced significantly compared with other type of Air-Cushion Vehicles (ACV). Also, it is possible to install conventional water propellers or waterjet propulsion, with rather smaller machinery space requirements compared to that for air propellers or fans used on ACVs. Furthermore, the SES can operate in modes of full displacement, partial air-cushion support, and full air-cushion support.

Predicting the overall performance of a SES is of paramount importance to support the design phase, as the motion of the ship can be affected by the interaction between the air, the cushion, the ship structure, the seals, the sea waves and the sea bottom in the shallow water region. Different approaches with different types of complexity and accuracy have been taken to cope with this type of analyses.

In the last decade, there have been extensive applications of Navier-Stokes models to naval hydrodynamics problems. In [60], an application for the calculation of the flow about a SES in still water, using a commercial Volume of Fluid model, has been presented. While, in [61], Mousaviraad et al. use an URANS solver for evaluating the maneuvering performance of a SES. While the outcome of the analyses is outstanding, the CPU-time reported in that thesis, makes this model quite unaffordable for being used during design stages.

Actually, it is a common consensus that solvers based on the Navier-Stokes equations are too expensive computationally speaking when it comes to simulate unsteady naval hydrodynamics problems. These sorts of problems can be more efficiently calculated using potential flow theory. This approach, jointly with the Stokes perturbation approximation, is widely used for analysis of seakeeping problems. In [62], Connell et al., use a boundary-element time-domain potential flow solver to calculate the multi-body seakeeping behavior of a T-Craft SES and a Large Medium-Speed Roll on/Roll off (LMSR) ship in different scenarios. While, in [63], the same computational solver is adapted to calculate the maneuvering properties of a SES.

Despite the complexity of the above referred SES computational models, none of them takes into account the seal dynamics, or the effect of free surface-seal interaction. However, the relevance of this 
interaction in the unsteady dynamics of a SES is well known $[64,65]$. The complexity of this phenomenon makes impossible to develop a theoretical background, and prompts many design parameters to be traditionally decided by empirical formulae [64]. Actually, only limited theoretical and computational models have been developed to analyze seal dynamics $[66,67,68]$.

This chapter focuses on the development of a computational model for the analysis of the complex and highly non-linear dynamic behavior of the seals in the interface between the air cushion and the water. The fluid solver used for this purpose uses the potential flow approach along with the streamline integration of the free surface. While this approximation is much simpler than using RANS computations, relevant outcomes can be obtained, allowing to significantly reducing computational time by 2 or 3 orders of magnitude even when computing on a regular desktop or laptop.

The developed fluid-structure interaction solver is based, on one side, on an implicit iteration algorithm, using a TCP/IP sockets link, able to communicate pressure forces and displacements of the seals at memory level and, on the other side, an innovative wetting and drying scheme able to predict the water action on the seals.

\subsection{Structural solver}

For the structural model of the, a computer code based on membrane and shell finite elements has been used. The membrane finite element used is a 3-noded membrane triangle. This element uses a total Lagrangian formulation and assumes a linear-elastic behavior of the material [69]. The kinematic description is formulated in large displacements and large deformations, making use of the right Cauchy-Green deformation tensor.

The shell finite element used is a corrotational 3-noded triangle shell element [70]. The core formulation of the bending part corresponds to a classical Discrete Kirchhoff plate Triangle (DKT). The core formulation of the membrane part corresponds to the Assumed Natural Deviatoric Strain (ANDES) approach [71]. The ANDES formulation represents a linear strain triangle with 3 corner nodes and 3 DOFs per node (two in-plane displacements and a drilling rotation).

An energy conserving/decaying time-stepping algorithm has been used for the dynamic analysis of the problem $[72,73,74]$. This approach ensures the stability in the non-linear geometric range.

In general, shell elements are the preferred option to model the seals behavior, because they can consider the actual flexural rigidity of the seals, despite being small. However, since the thickness of the seals is very small, numerical instabilities might appear since the tangent matrix is poorly conditioned by the large ratio between membrane and flexural rigidity of the seals. In these cases, the only solution is to neglect the flexural rigidity by using membrane elements. In a dynamic analysis, 
the membrane elements no longer have these problems since the tangent stiffness matrix is properly conditioned by the mass matrix.

\subsection{Coupled fluid-structure solver}

\subsubsection{Free surface-seal interaction (FSSI)}

Modelling the behavior of the seals of aircushion vehicles is not a trivial issue, due to the complex interaction of the seals with the free surface. In this thesis, a new algorithm for handling the free surface-seal interaction problem is formulated. It is based on finding an equivalent pressure field to be applied over the free surface such that the elevation of this one is limited by the location of the seal. That is to say, the seal acts as an upper limit for the free surface elevation.

The free surface boundary conditions are applied in different ways depending on whether or not the free surface is in contact with the seal. If the free surface is not in contact, the boundary conditions are applied as if there is no seal, but if there is contact, the implementation will be different in order to ensure that the free surface does not penetrate the seal, and the required free surface pressure to fulfil this condition is calculated and applied as a boundary condition.

The free surface node where the algorithm is to be applied is said to be dry if the seal is not in contact with the free surface at that location, and wet if it is. Figure 47 illustrates the wet and dry concepts.

The main challenge for an algorithm like this is to be capable of capturing when a node goes from dry to wet and vice versa, as well as estimating the pressure field on the wet nodes. Figure 48 shows the wetting and drying iterative algorithm. For a dry node, the implementation of both the kinematic and dynamic boundary condition is the same as for any other node not interacting with the seal. However, for wet nodes, the free surface boundary condition (3-55) is imposed forcing the free surface elevation to match the seal elevation, $\mathrm{H}_{\text {seal }}$, and ensuring that there is no flow across the seal. These two conditions are represented by the following equations:

$$
\begin{aligned}
& \eta_{\text {wet }}^{n+1}=H_{\text {seal }}^{n+1}, \\
& \left(\partial_{z} \phi\right)^{n+1}=2\left(\frac{\eta^{n+1}-\eta^{n}}{\Delta t}+\left|U^{n+1 / 2}\right|\left(\delta_{L} \eta\right)^{n+1 / 2}\right)-\left(\partial_{z} \phi\right)^{n},
\end{aligned}
$$

where $\eta$ is the free surface elevation, $\phi$ is the velocity potential, $H_{\text {seal }}$ is the seal height, $\Delta t$ is the time step, $U$ is the flow field, and $\delta_{L}$ represents the numerical derivative along the streamline. $\left(\partial_{z} \phi\right)^{n+1}$ is to be imposed as a Neumann condition instead of imposing $\phi^{n+1}$ as Dirichlet condition. 


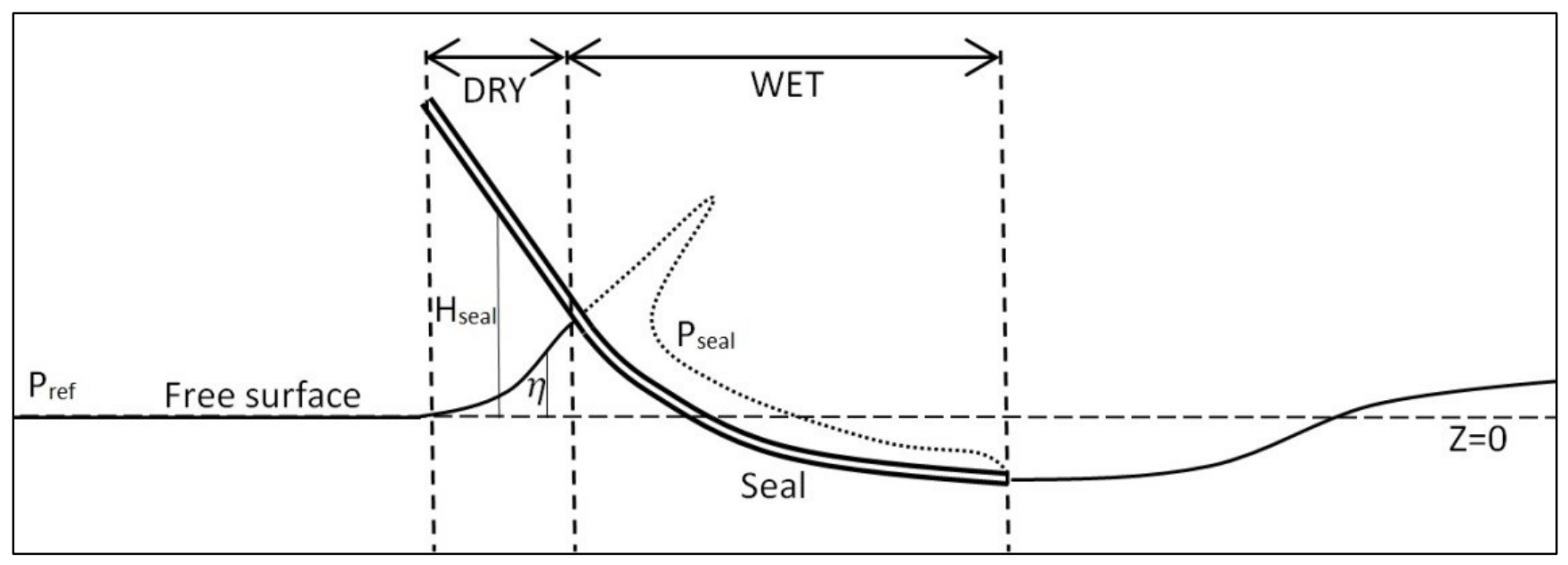

Figure 47: Wet and dry seal regions for free surface boundary condition implementations.

On the one hand, the switch from being a dry node to becoming a wet node is identified via the kinematic $\mathrm{BC}$ through the condition $\eta_{w e t}^{n+1}>H_{\text {seal }}^{n+1}$. On the other hand, the switch from being a wet node and becoming a dry one is carried out by comparing the dynamic pressure with the reference free surface pressure. The dynamic pressure on wet nodes is obtained via the dynamic BC (Eq. (3-54)):

$$
\begin{gathered}
P_{w e t}^{n+1}=-\rho\left[\frac{\phi^{n+1}-\phi^{n}}{\Delta t}+\left|U^{n+1}\right| \cdot\left(\delta_{L} \phi\right)^{n+1}-\frac{1}{2}\left(\nabla_{h} \phi\right)^{n+1} \cdot\left(\nabla_{h} \phi\right)^{n+1}\right. \\
\left.+\frac{1}{2}\left(\partial_{z} \phi\right)^{n+1}\left(\partial_{z} \phi\right)^{n+1}+g \eta^{n+1}\right]
\end{gathered}
$$

where $P_{w e t}$ is the free surface pressure, or pressure on the seal, for a wet node.

It was found that when the seal is interacting with the free surface, the term $\phi_{z}$ might become of the same order of magnitude than $U$. Hence the term $\frac{1}{2} \phi_{z}^{2}$ has been kept when calculating the pressure on a free surface node in contact with the seal.

\subsubsection{Free surface-flexible seas coupling algorithm}

The fluid-structure coupling is performed by an implicit interfield iteration algorithm [75], based on a Block Gauss-Seidel method [76]. For the i-th iteration of the current time step n, let $\phi^{n, i}, \eta^{n, i}$, $p^{n, i}$ be the vectors of the velocity potential, free surface elevation and pressure in the corresponding nodes of the fluid, $x^{n, i}$ the vector containing the three components of the displacement of the nodes of the seals structure, with $\mathrm{x}_{\mathrm{k}}^{\mathrm{n}, \mathrm{i}}$ the vector of the $\mathrm{k}$-th component of the displacement field, and $H_{\text {seal }}^{n, i-1}$ the vector of the elevation field of the seal nodes, then the fluid-structure interaction algorithm, can be written as: 


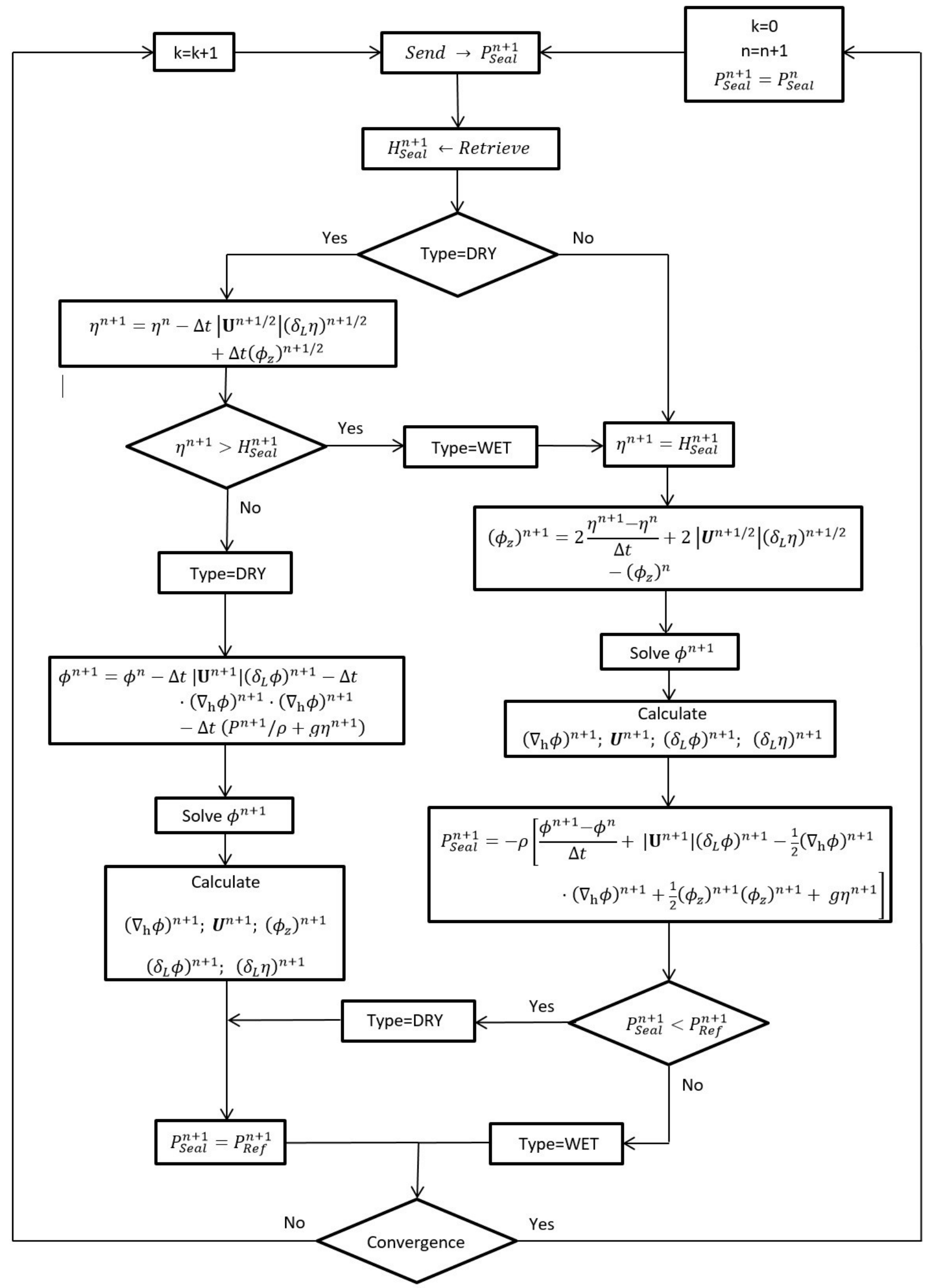

Figure 48: Wetting and drying algorithm. 


$$
\begin{gathered}
\left(p^{n, i}, \phi^{n, i}\right)=F^{p}\left(\phi^{n, i-1}, \eta^{n, i-1}, H_{\text {seal }}^{n, i-1}, \eta^{n-1}, \phi^{n-1}\right), \\
x^{n, i}=S^{q}\left(x^{n, i-1}, p^{n, i}, x^{n-1}, x^{n-2}\right), \\
H_{\text {seal }}^{n, i-1}=H_{\text {seal }}^{0}+x_{k}^{n, i},
\end{gathered}
$$

where $F, S$ represent the fluid and solid solver, and $\mathrm{p}$ and $\mathrm{q}$ are the number of internal iterations done within the non-linear loop of each solver. This way, the pressure field computed in the hydrodynamic solver is sent to the structural solver to compute the seals deformation. The resulting displacements are used to compute the new seal elevation on the fluid interface, and then calculate the pressure field for the following iteration.

Since the hydrodynamics and structural solvers used in this work are independent, the strategy developed to communicate both is based on the interchange of information at memory level by means of TCP-IP sockets. This way, only minor adaptations of the solvers were required. A pre-existing communication library has been used for this purpose. Furthermore, this library is capable of interpolating data between the boundary meshes of the fluid and solid interfaces (see Figure 49). The algorithm to interpolate data between meshes can be summarized as follows:

a. Two octree structures are created to store the seal mesh elements and the elements of the fluid patch affected by the seal deformation.

b. For every node of the seal mesh, the corresponding octree is used to identify the set of fluid elements where the node is likely to be. For every element of the set, it is checked whether or not the seal node rests on it. As a result of this search, the fluid element in contact with the seal node is selected.

c. Step b) is repeated for every node of the elements of the fluid patch affected by the seal deformation.

d. The shape functions of the selected elements are used to interpolate the information from the fluid to the structure and vice-versa. This information is stored in two matrixes; $I^{F S}$, that allows interpolating the pressure field from the fluid to the seal structure, and $I^{S F}$, that interpolates the seal deformation field to be used as boundary condition for the fluid flow solver.

Then, the fluid-structure interaction algorithm reads:

$$
\begin{gathered}
\left(p^{n, i}, \phi^{n, i}\right)=F^{p}\left(\phi^{n, i-1}, \eta^{n, i-1}, H_{\text {seal }}^{n, i-1}, \eta^{n-1, i-1}, \phi^{n-1}\right), \\
\check{p}^{n, i}=I^{F S}\left(p^{n, i}\right), \\
x^{n, i}=S^{q}\left(x^{n, i-1}, \check{p}^{n, i}, x^{n-1}, x^{n-2}\right), \\
\check{x}^{n, i}=I^{S F}\left(x^{n, i}\right), \\
H_{\text {seal }}^{n, i-1}=H_{\text {seal }}^{0}+\check{x}_{k}^{n, i} .
\end{gathered}
$$

Even though the iterations within each time step lead to the monolithic solution of the coupled problem, sometimes relaxation techniques are combined with this method to ensure the convergence 
of the coupling scheme. In this thesis, a relaxation method based on Aitken's acceleration has been adopted [42]. Let $\omega_{i}$ be the optimal relaxation parameter, obtained via Aitken's method, the resulting algorithm can be written as:

$$
\begin{gathered}
\left(p^{n, i}, \phi^{n, i}\right)=F^{p}\left(\phi^{n, i-1}, \eta^{n, i-1}, H_{\text {seal }}^{n, i-1}, \eta^{n-1, i-1}, \phi^{n-1}\right), \\
\hat{p}^{n, i}=\left(1-\omega_{i}\right) \hat{p}^{n, i-1}+\omega_{i} p^{n, i}, \\
\check{p}^{n, i}=I^{F S}\left(\hat{p}^{n, i}\right), \\
x^{n, i}=S^{q}\left(x^{n, i-1}, \check{p}^{n, i}, x^{n-1}, x^{n-2}\right), \\
\check{x}^{n, i}=I^{S F}\left(x^{n, i}\right), \\
H_{\text {seal }}^{n, i-1}=H_{\text {seal }}^{0}+\check{x}_{k}^{n, i} .
\end{gathered}
$$

As stated above, the free surface-seals interaction algorithm is based on finding an equivalent pressure field to be applied over the free surface such that the elevation of this one is limited by the location of the seal. For this purpose, the elevation of the seal over the reference free surface is required. The algorithm (9-5) assumes that the variation of this elevation is only due to the vertical displacements of the structure. This is a good enough approximation to predict the forces of the water on the seals and the free surface elevation in the seals region.

\subsection{Validation of FSSI algorithm with flexible bow seals}

\subsubsection{Validation: experiments at University of Michigan}

Zalek and Doctors [77] carried out an extensive experimental study of free surface and flexible seal interaction at the University of Michigan. Some of these data have been already used for validation purposes against numerical results based on a Smoothed Particle Hydrodynamics approach [78]. Figure 50 sketches the basic arrangements of the experiments, such as seal length, location of stiffeners and cables, and undeflected seal angle. Table 23 provides the seal properties used for the experiments [78].

In this thesis, the experimental cases shown in Table 24 were analyzed with the proposed fluidstructure interaction model. The test runs were simulated modelling the left half part of the seal and tank, using the actual width and depth of the channel of University of Michigan. The seals were modelled with shells elements.

Table 25 provides the main characteristics of the computational mesh used for the simulation, as

well as the length of the numerical channel used. For test run 45, a larger channel was necessary to avoid upstream and downstream interactions with the inlet and outlet boundaries respectively. 


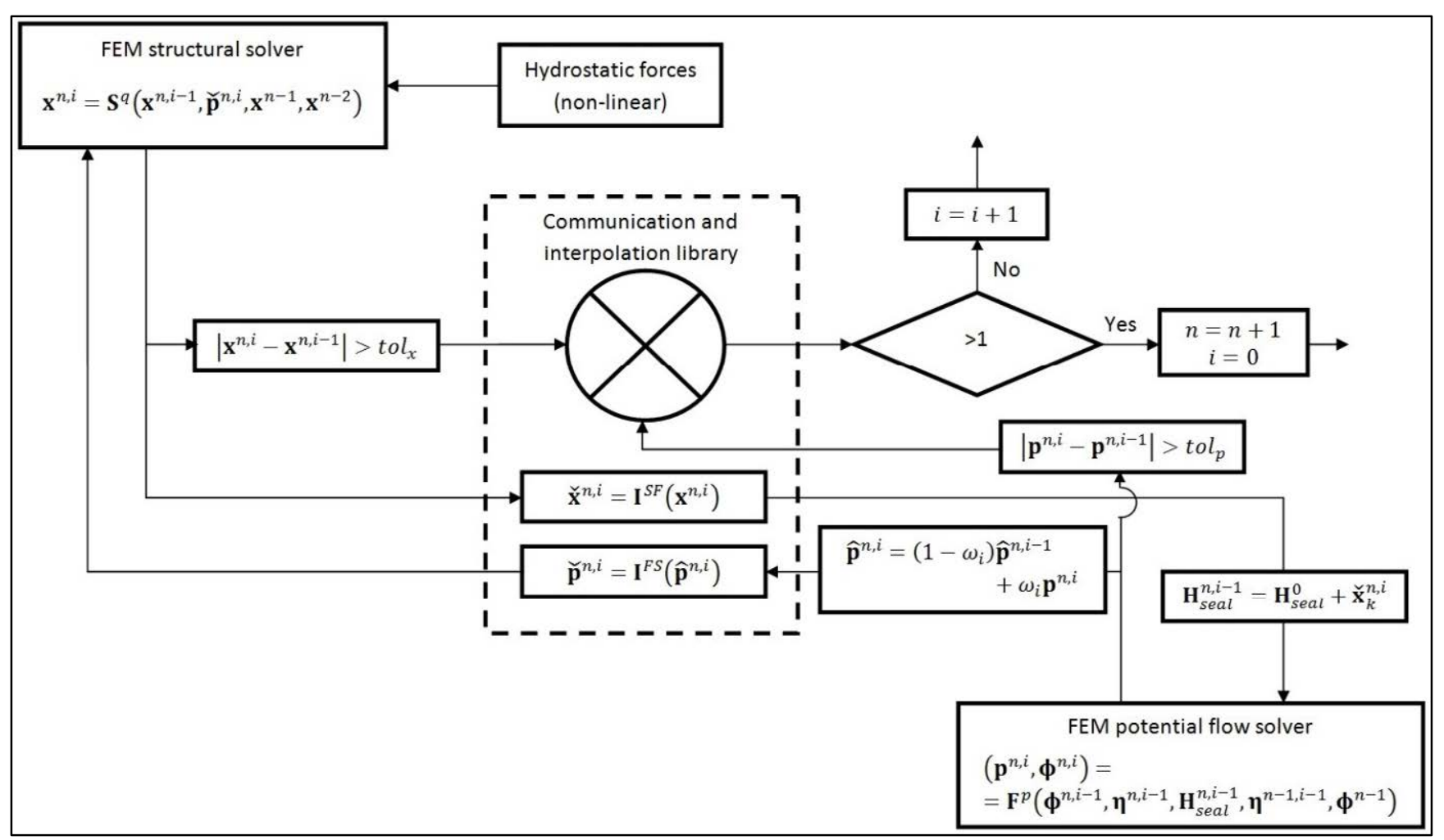

Figure 49: Fluid-structure interaction algorithm.

In the experiments, three stiffeners (rods) were fixed to the seal to reduce the transversal deformation. Since data regarding the properties of such stiffeners were not available, they were simulated by introducing very stiff beams at the same locations, but weightless, assuming that the ones used in the experiments were light enough to avoid significant perturbation in the seal behavior. Furthermore several cables were attached to the stiffeners to avoid the forward displacement of the seal due to the internal pressure. Since there is no available information about the length and exact disposition of these cables, they were simulated in the computational model by a boundary condition, constraining the longitudinal displacement of the points of the seal where the cables were attached.

Figure 51 shows the quasi-steady deformed front seal for test run number 10. Figure 52 compares the average seal deformation obtained numerically and experimentally. Although the computational results do not exactly match the experimental ones, they agree well in terms of the main trends under different conditions of velocity, seal immersion and internal pressure. In addition, the differences are likely within the uncertainty range of the data (not reported), since the unsteady and three-dimensional effects are relevant in most of the cases studied.

Figure 53 provides the top view of the hydrodynamic pressure distribution acting on the seal due to the free surface seal interaction. It can be observed that while in some cases the distribution is almost uniform in the transverse direction that is not the case in the other ones, resulting in relevant three dimensional effects, as mentioned before. 


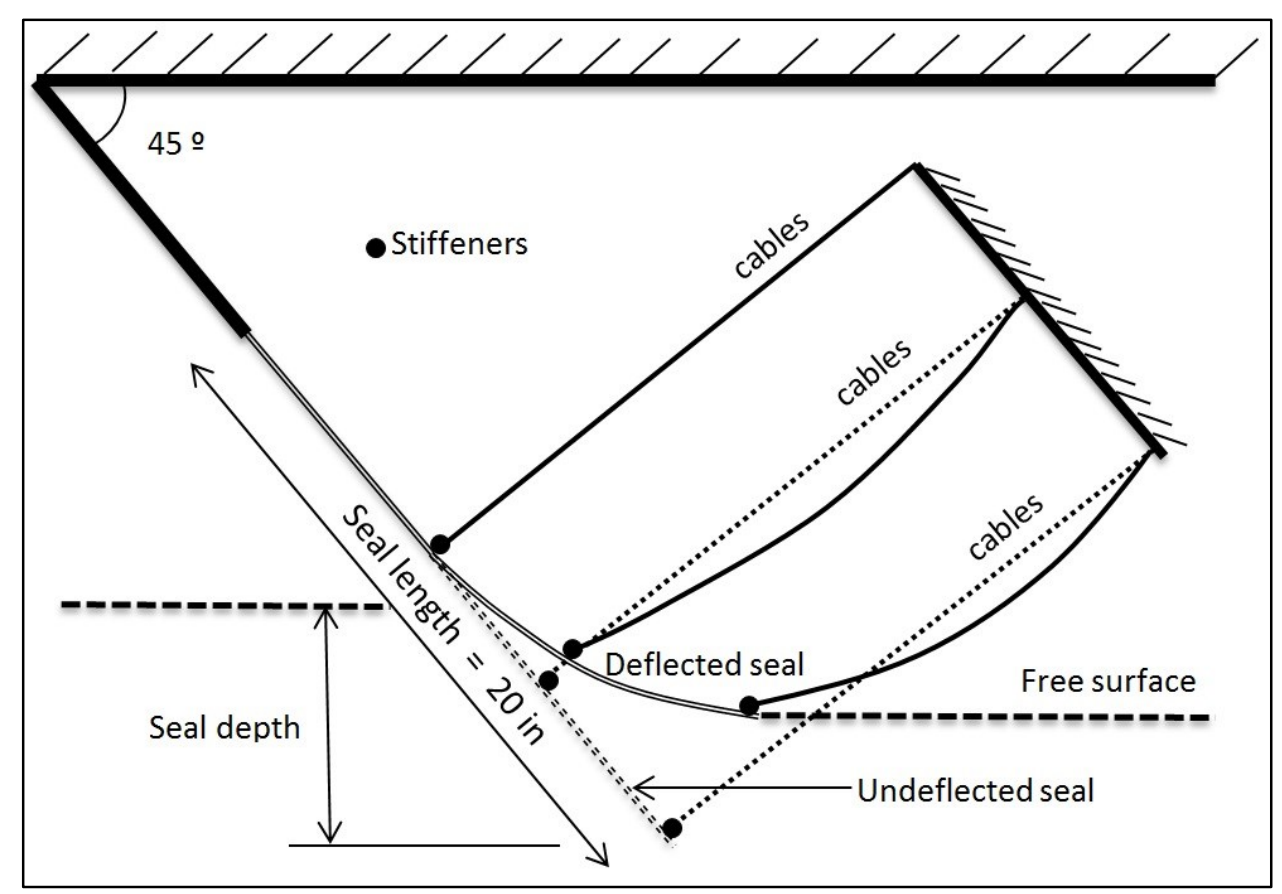

Figure 50: Experiments setup at University of Michigan [77].

Table 23: Seal properties

\begin{tabular}{|c|c|}
\hline Thickness & $3.175 \mathrm{~mm}$ \\
\hline Young Modulus & $12.74 \mathrm{MPa}$ \\
\hline Poisson coefficient & 0.25 \\
\hline Density & $1107 \mathrm{~kg} / \mathrm{m} 3$ \\
\hline
\end{tabular}

Table 24: Test run particulars

\begin{tabular}{|c|c|c|c|c|c|c|}
\hline \multirow{2}{*}{ Test run [77] } & \multicolumn{2}{|c|}{ Seal Immersion } & \multicolumn{2}{c|}{ Velocity } & \multicolumn{2}{c|}{ Internal Pressure } \\
\cline { 2 - 7 } & $\mathbf{( i n )}$ & $\mathbf{( m )}$ & $\mathbf{( f t / s )}$ & $\mathbf{( m / s )}$ & (in. H2O $)$ & $\mathbf{( P a )}$ \\
\hline $\mathbf{1}$ & 9 & 0.2286 & 6 & 1.8288 & 1.42 & 353.3 \\
\hline $\mathbf{1 0}$ & 9 & 0.2286 & 8 & 2.4384 & 1.01 & 251.3 \\
\hline $\mathbf{1 1}$ & 9 & 0.2286 & 8 & 2.4384 & 1.91 & 475.3 \\
\hline $\mathbf{1 6}$ & 9 & 0.2286 & 8 & 2.4384 & 4.24 & 1055 \\
\hline $\mathbf{3 2}$ & 7.5 & 0.1905 & 6 & 1.8288 & 1.28 & 318.5 \\
\hline $\mathbf{4 5}$ & 7.5 & 0.1905 & 9 & 2.7432 & 4.01 & 997.8 \\
\hline
\end{tabular}

Table 25: Computational domain and numerical particulars

\begin{tabular}{|c|c|c|c|c|c|c|}
\hline \multirow{2}{*}{$\begin{array}{l}\text { Test } \\
\text { run }\end{array}$} & \multicolumn{2}{|c|}{ Seal } & \multicolumn{2}{|c|}{ Water volume } & \multirow{2}{*}{$\begin{array}{c}\text { Time step } \\
\text { (s) }\end{array}$} & \multirow{2}{*}{$\begin{array}{c}\text { Domain } \\
\text { length } \\
\text { (m) }\end{array}$} \\
\hline & $\begin{array}{c}\text { Number of } \\
\text { Nodes }\end{array}$ & $\begin{array}{c}\text { Number of } \\
\text { Elements }\end{array}$ & $\begin{array}{c}\text { Number of } \\
\text { Nodes }\end{array}$ & $\begin{array}{c}\text { Number of } \\
\text { Tetrahedron }\end{array}$ & & \\
\hline 1 & 234 & 424 & 58136 & 297188 & 0.002 & 5.3 \\
\hline 10 & 234 & 424 & 58315 & 297888 & 0.001 & 5.3 \\
\hline 11 & 234 & 424 & 58315 & 297888 & 0.001 & 5.3 \\
\hline 16 & 234 & 424 & 58240 & 297643 & 0.002 & 5.3 \\
\hline 32 & 234 & 424 & 56112 & 287493 & 0.002 & 5.3 \\
\hline 45 & 234 & 424 & 80325 & 419569 & 0.001 & 15.3 \\
\hline
\end{tabular}




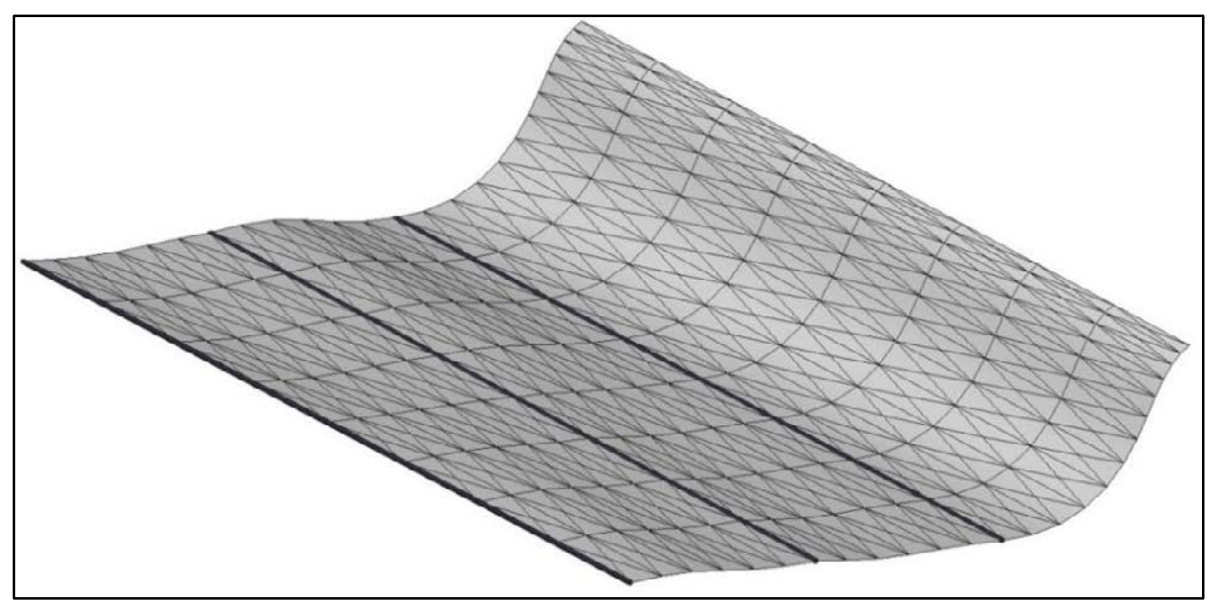

Figure 51: Seal deformation and structural mesh for University of Michigan test run 10 [77].

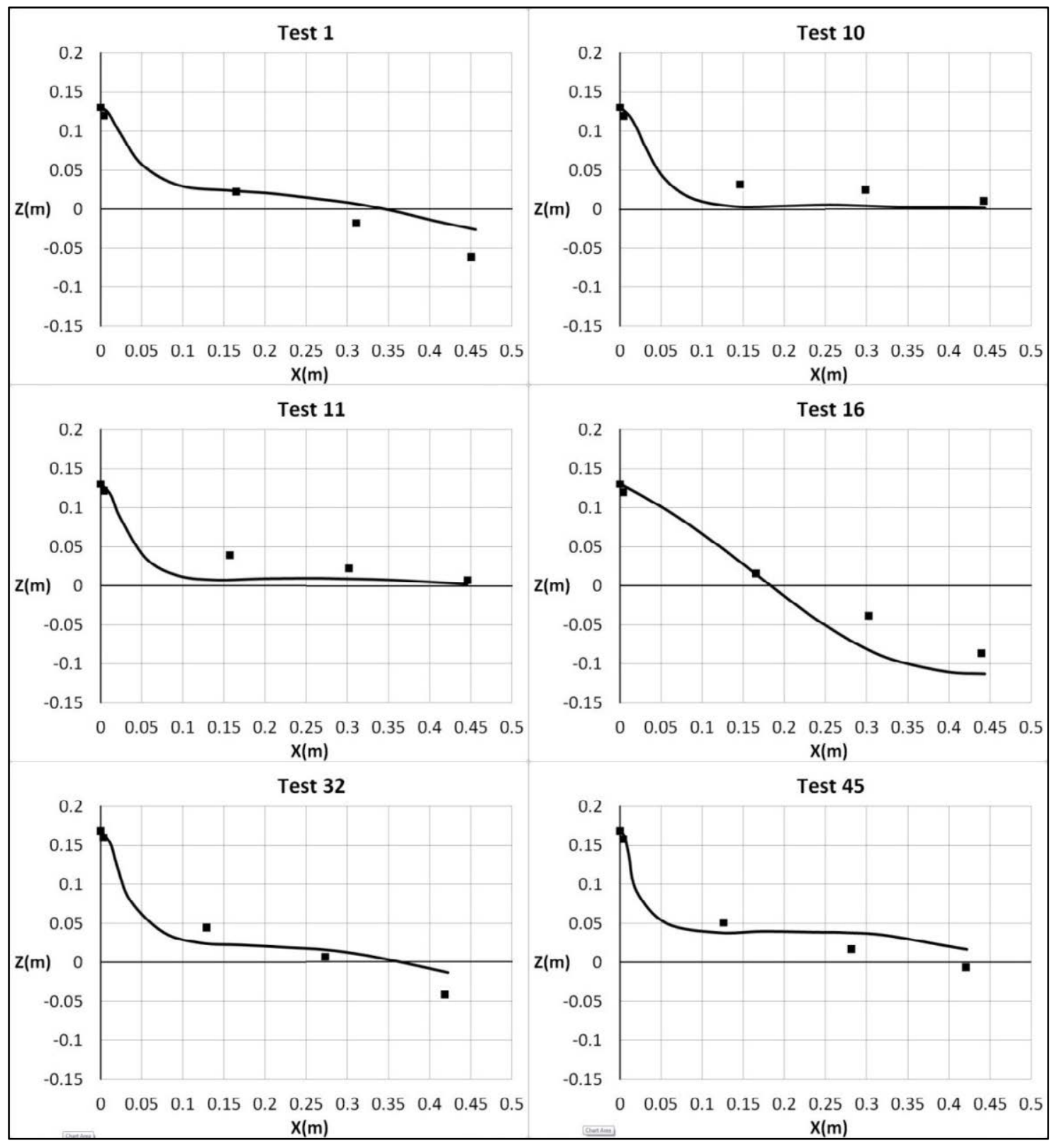

Figure 52: Seal deformation: University of Michigan experimental results (squares) [77] versus numerical (solid line). 


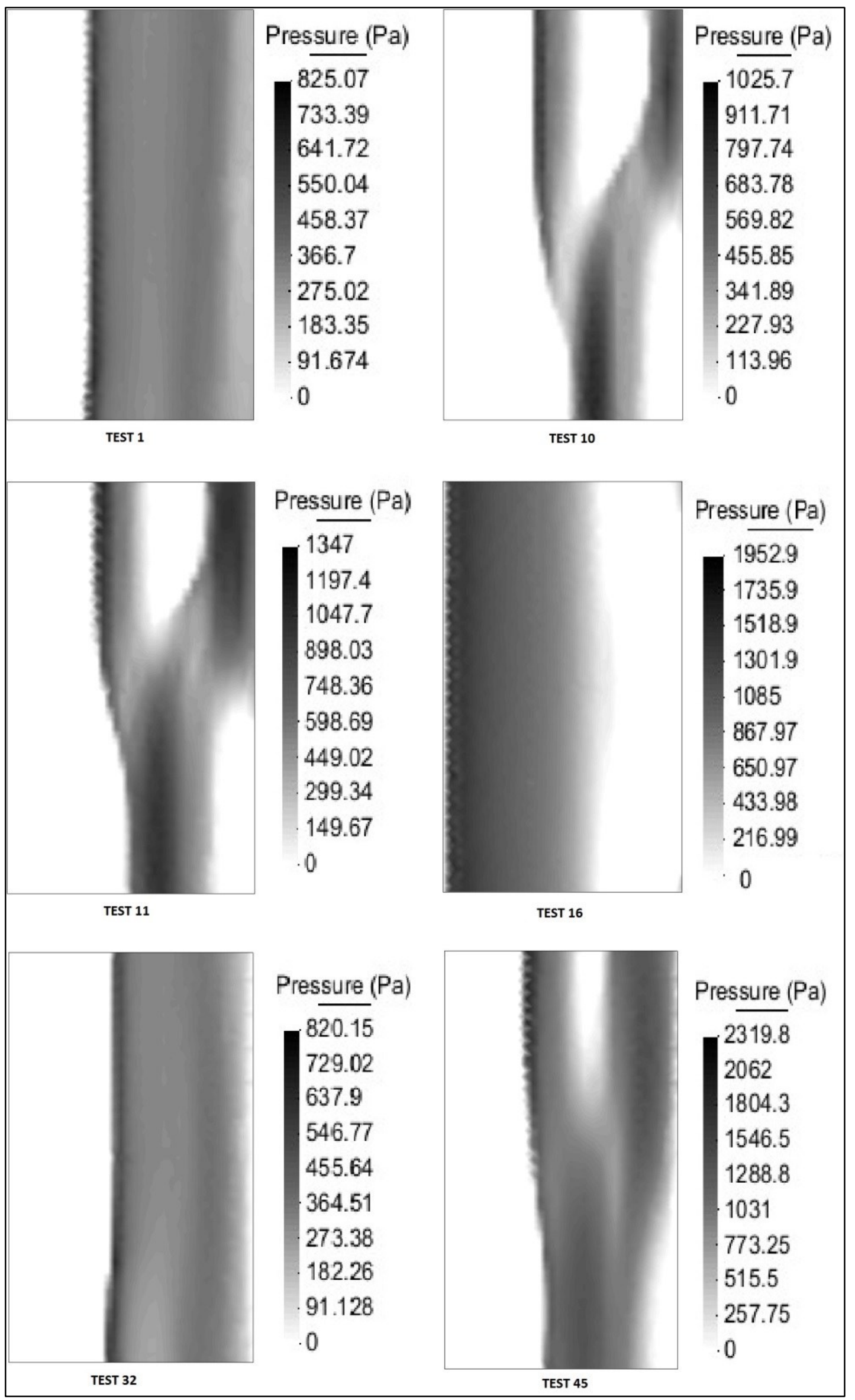

Figure 53: Top view of pressure distribution over seal for University of Michigan selected test cases. 


\subsubsection{Validation: experiments at the U.S Navy's Large Cavitation Channel (LCC)}

Wiggins et al. [79] developed a large scale testing platform for finger bow seals at the U.S. Navy's Large Cavitation Channel. The purpose of those tests was to provide experimental results to be used for validation purposes of analytical/numerical approaches to the problem. Figure 54 sketches the model setup at the LCC facilities.

The seal material used is the same one that the one used in University of Michigan experiments and described in the previous section. In this case, a membrane model was used for the computational analysis of the structure, since preliminary tests with a shell model showed an unstable behavior, attributed to the poor condition of the tangent matrix.

With regards to the test condition, a free stream flow of $6 \mathrm{~m} / \mathrm{s}$, seal immersion of $15 \mathrm{~cm}$, and internal pressure of $1.5 \mathrm{KPa}$ [79] was considered. Other data needed for the setup of the case under study are provided in Table 26.

Table 27 provides the main characteristics of the computational mesh used for the simulation, as well as the length of the numerical channel used. Figure 55 shows the computational mesh used for solving the fluid problem.

Figure 56(a) shows the steady seal deformation obtained in the experiments under the abovementioned test conditions. Figure 56(b), (c) and (d) show seal deformation obtained by the numerical approach proposed in this work under the same test conditions. Free surface elevation at the seal and aircushion areas are shown in Figure 57(a). A top view of the pressure distribution over finger seals is shown in Figure 57(b). The bow seals deformation obtained in this analysis agrees well qualitative with the experimental information available in reference [79]

\subsection{Summary}

This chapter has documented the advances in the development of a fluid-structure interaction algorithm for evaluation of a SES seal dynamics. The fluid solver developed for this purpose uses a potential flow approach along with a stream-line integration of the free surface. The work focuses on the free surface-structure interaction algorithm to simulate complex and highly non-linear dynamic behavior of the seals in the interface between the air cushion and the water.

The developed fluid-structure interaction solver is based, on one side on an implicit iteration algorithm, communicating variables at memory level, and on the other side on an innovative wetting and drying scheme. 
Special care has to be taken to ensure the convergence of the fluid-structure interaction algorithm due to the highly flexible and non-linear nature of the seal. We found that a relaxation method based on Aitkens' methods worked well under all the cases analyzed.

Finally, different validation and demonstration cases have confirmed the validity of the approach to study the dynamic behavior of the seals.

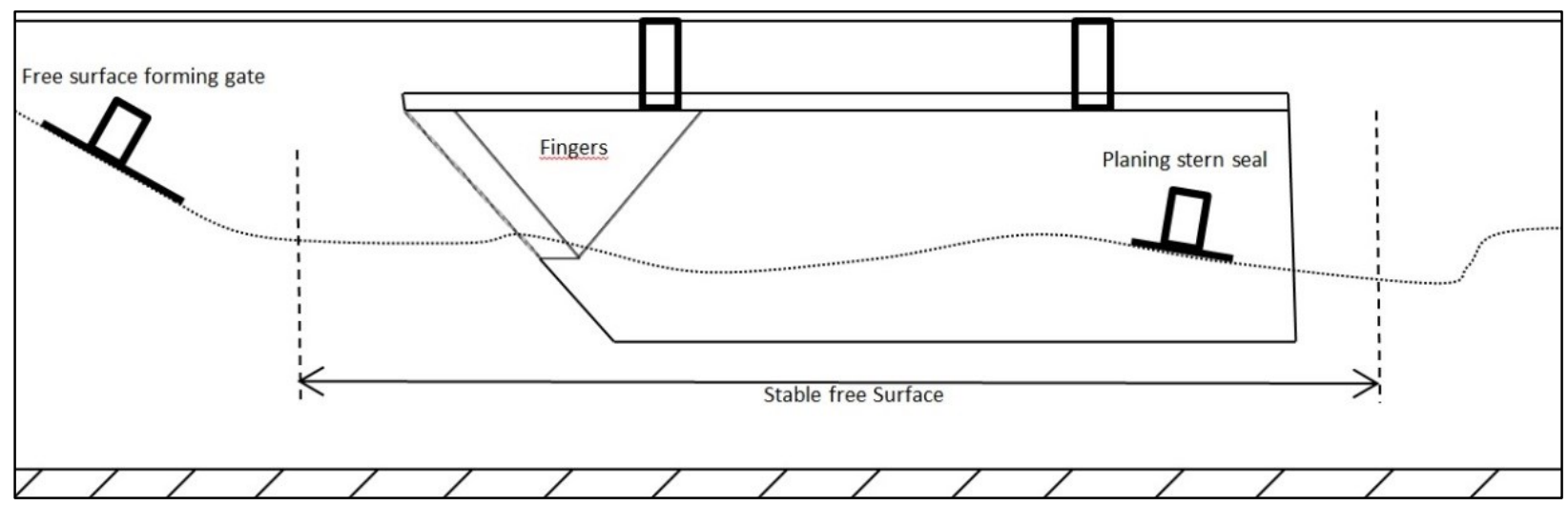

Figure 54: LCC model setup sketch [79]

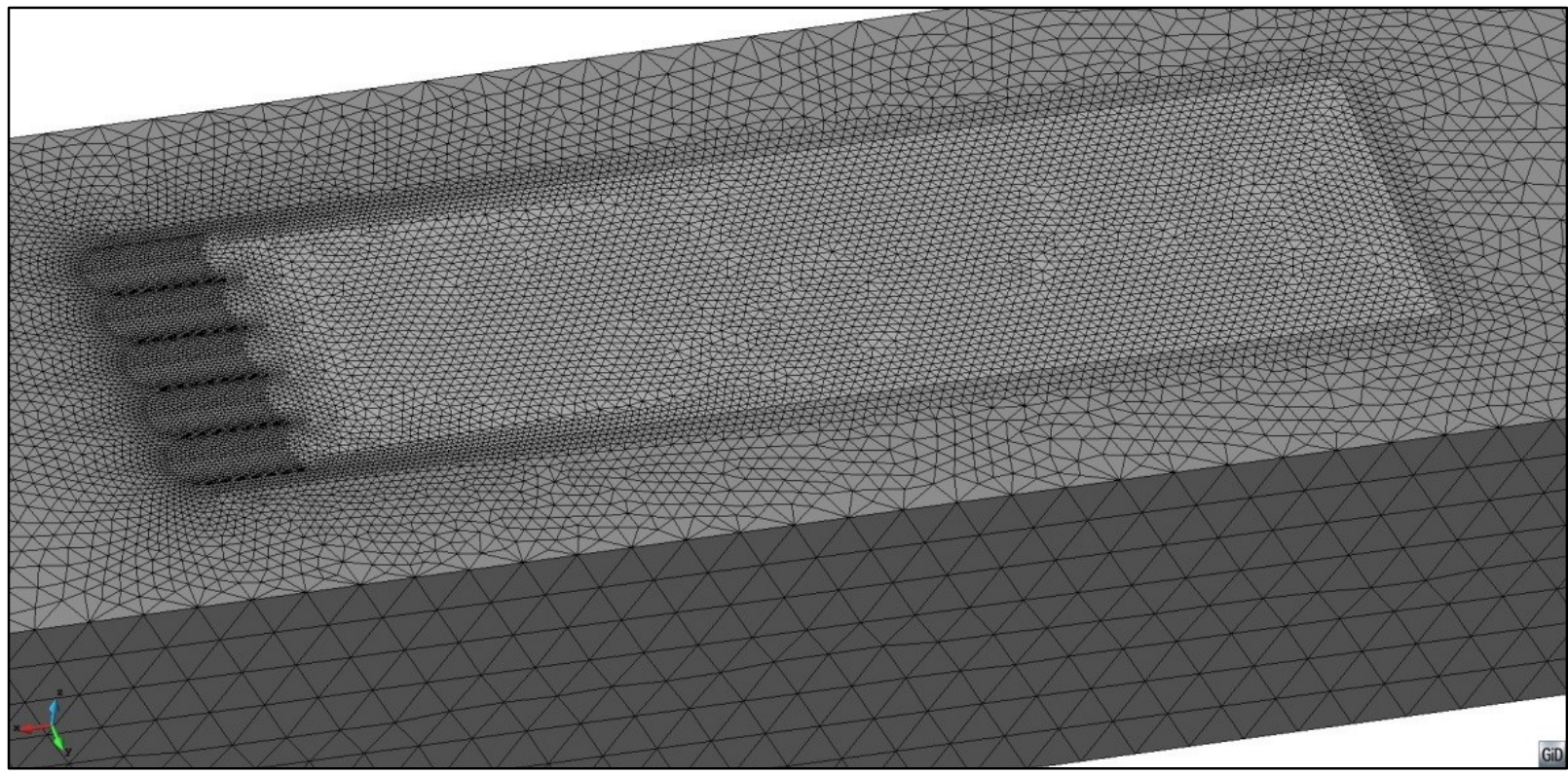

Figure 55: Computational mesh for potential flow solver for LCC test case [79].

Table 26: Tests particulars [79]

\begin{tabular}{|c|c|}
\hline Breadth of cushion (m) & 1.52 \\
\hline Sidewall depth $(\mathbf{m})$ & 1.68 \\
\hline Sidewall draft at zero immersion $(\mathbf{m})$ & 0.42 \\
\hline Channel width $(\mathbf{m})$ & 3.05 \\
\hline Number of Fingers 5 & 5 \\
\hline Finger height (m) & 1.00 \\
\hline Finger width $(\mathbf{m})$ & 0.31 \\
\hline Bow seal angle $\left.\mathbf{(}^{\mathbf{o}}\right)$ & 50.00 \\
\hline
\end{tabular}


Table 27: Computational domain and numerical particulars

\begin{tabular}{|c|c|c|}
\hline \multirow{2}{*}{ Fingers } & Number of Nodes & 7023 \\
\cline { 2 - 3 } & Number of Elements & 13436 \\
\hline \multirow{2}{*}{ Water domain } & Number of Nodes & 120942 \\
\cline { 2 - 3 } & Number of Tetrahedron & 659687 \\
\hline \multicolumn{2}{|c|}{ Time step (s) } & 0.0005 \\
\hline \multicolumn{2}{|c|}{ Domain length (m) } & 21 \\
\hline
\end{tabular}

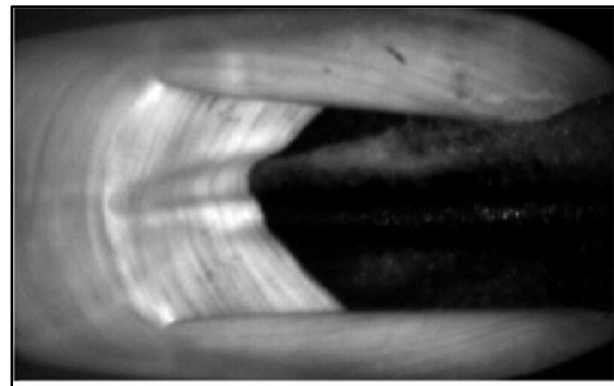

(a)

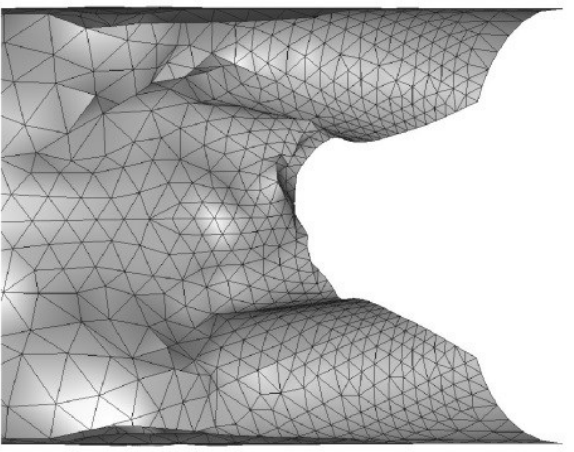

(c)

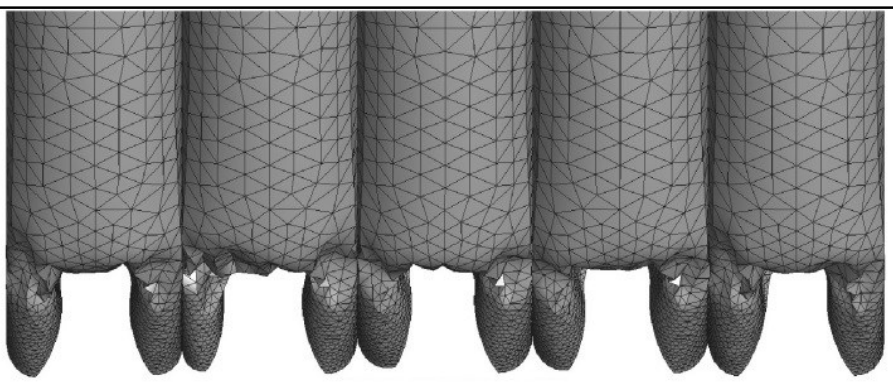

(b)

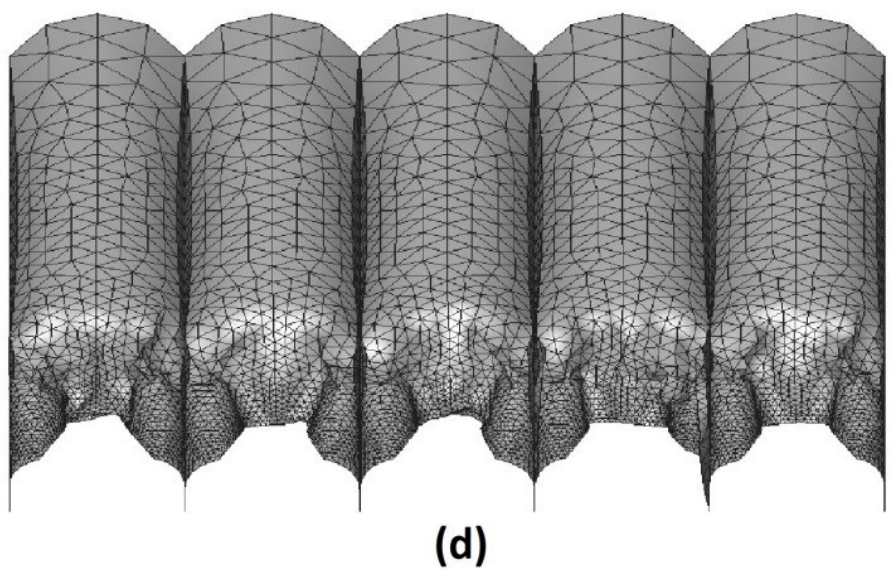

(d)

Figure 56: Experimental results (a) [79] versus numerical results (b), (c) and (d). Picture obtained in the experimental tests reported in [80] (a); Front view of deformed fingers (b); deformed finger detail (c); top view (d). 


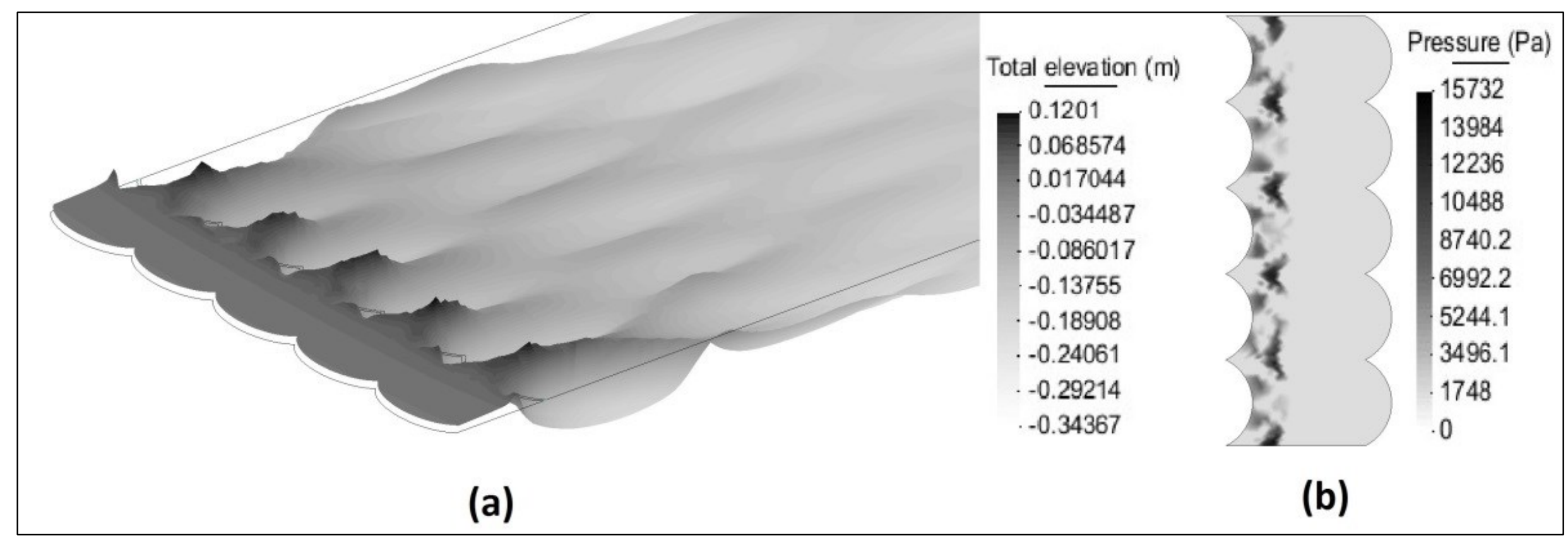

Figure 57: Numerical results for LCC test case [79]: free surface elevation (a) and top view of pressure distribution on fingers (b). 


\section{Chapter 10. ANALYSIS OF COUPLED SEAKEEPING - SLOSHING PROBLEMS BY FEM- SPH COUPLING}

\subsection{Introduction}

The aim of this chapter is to carry out numerical simulations in the time domain of seakeeping problems taking into account internal flows in tanks, including sloshing. To achieve this aim, a Smooth Particle hydrodynamics ( $\mathrm{SPH}$ ) solver is coupled in the time domain to the FEM diffraction-radiation solver presented in sections $0-2.3$.

There are a series of marine operations and/or navigation conditions in which the coupling between internal flow in tanks and seakeeping dynamics can be crucial in configuring the global dynamic response of the vessel. The assessment of such coupling effects may be thus extremely important in order to estimate the viability of certain operations as well as the risks associated to specific load conditions during navigation. Such navigation conditions and/or operations include: those carried out by offshore vessels, equipped with anti-roll tanks, commonly in the oil\&gas industry, consisting of deploying piping systems, cables, drilling equipment, etc... (see e.g. [81]); those referred to the offloading of oil or liquefied natural gas (LNG) from an Floating Production, Storage and Offloading (FPSO), bunkering vessel or a floating LNG (FLNG) to a shuttle, regular vessel or LNG carrier, respectively, in side-by-side configurations (see e.g. [82]); the transport in partially filled tanks of such LNG or its storage in FLNG tanks (see [83] for a review on FLNG vessels hydrodynamics).

Due to their large economic cost and much larger penalties in case of any unexpected problem, the planning of above mentioned operations or the definition of filling level thresholds of tanks require a deep understanding of the coupled dynamics between the vessel and the internal flows. In order to achieve such understanding it is important to notice that while the external dynamics can be usually resolved in relatively large time scales, the internal dynamics may incorporate violent sloshing flows, which requires resolving complex free-surface dynamics with much shorter time scales. In addition, when wave excitation is present, for low wave amplitudes the motions are usually linear with amplitude and frequency, while for moderate or large wave amplitudes the response can be extremely nonlinear. For these reasons, in order to simultaneously resolve the seakeeping and internal flow dynamics, different types of solvers are typically used for each of them. For instance, Zhao et al. [84] used a 
frequency domain linear BEM approach to obtain hydrodynamic coefficients and wave forces, which is coupled in the time-domain with a nonlinear potential solver for the internal dynamics. Another example is the commonly cited reference of Kim et al. [85] who also resort to frequency domain calculations and resolve the internal flow modelled with Euler equations, with a finite difference scheme. With the same technique for ship motions Li et al. [86] solved the internal flows with the open-source volume of fluid based solver OpenFOAM.

In order to solve the seakeeping dynamics, time domain diffraction-radiation solvers based on FEM can be an option [87]. Their capability to naturally incorporate non-linearities is larger than those timedomain solvers based on frequency domain pre-calculations such as the ones used by [84], [85] or [86]. Those solvers have been successfully used, for instance, with coupled mooring models [87] and for side-by-side problems [88]. However seakeeping FEM based solvers, to the author's knowledge, have not yet been used to model coupled sloshing and ship motions. Due to the onset of violent sloshing, internal dynamics can be extremely complex and therefore computationally expensive to resolve. In this context, SPH solvers can be a competitive option since, on the one hand, they are able to cope with extremely non-linear and fragmented free-surface flows, and on the other, they are easily parallelized with cost competitive graphic card processing units.

SPH solvers have been successfully used in the past for solving coupled dynamics of single angular motion coupled with sloshing problems [89,90,91], and their parallelization with GPUs has recently been subject of significant attention (see e.g. $[92,93,94,95])$. In this work, the internal flow dynamics is solved using the pre-existing code AquaGPUSPH [96].

This chapter is organized as follows: section 10.2 introduces the internal flow and wave diffractionradiation solvers; section 10.3 describes the rigid body dynamics solver; section 10.4 focuses on the details of the coupling algorithm, as well as in the communication between solvers; section 10.5 presents a validation study comparing the results obtained in this work against experimental data available for three different case scenarios; finally, section 10.6 provides summary of this chapter.

\subsection{Internal flow solver: AQUAgpusph}

AQUAgpusph [96] is a recently released free 3D SPH solver, licensed under GPLv3, and accelerated with OpenCL. SPH is a meshless numerical method that was developed in the seventies and first applied in the nineties to free-surface flows [97], with consistency of the operators in these contexts demonstrated by $[98,99,100]$. A recent review on the SPH method can be found in [101]. AQUAgpusph uses the weakly compressibility assumption [97] in order to model incompressible flows. Its source code is available for downloading at [102]. AQUAgpusph validation is thoroughly 
documented in $[96,103]$. In [96] the solver is validated for impact pressure in dam-break flows and for the same TLD case studied by $[89,90,91]$.

\section{$10.3 \quad$ Inserting SPH loads}

Since the body dynamics solver is implicit and non-linear, it requires iterating in order to achieve convergence within each time step (the reader is referred to 0 for the details of the body dynamics solver). As shown in Figure 58, there are two iterative loops within each time step, and $\overline{\bar{M}}$ is the body mass matrix, $X$ is the body position vector, $\dot{X}$ is the body velocity vector, $\ddot{X}$ is the body acceleration vector, $F_{0}$ are the external forces other than the hydrodynamics and $\mathrm{SPH}$ ones, $F_{H}$ are the hydrodynamics ones, and $F_{S P H}$ are the internal tanks vector of forces and moments computed by the SPH solver. Superscript " $n$ " represents the current time step, " $k$ " represents the k-th iteration of the solver loop, and " $l$ " represents the 1-th iteration of the body dynamics loop.

On the one hand, the outer loop iterates over the hydrodynamics solver obtained from the diffraction-radiation problem. On the other hand, the inner loop iterates over the body dynamics solver updating any other loads acting on the body dynamics.

\subsection{Coupling scheme}

\subsubsection{Communication issues}

The seakeeping and internal flow solvers are independently developed and compiled. Therefore, the development and implementation of a communication strategy between them is a key point.

The general idea of the designed coupling scheme is trivial: body movements calculated by the seakeeping solver are sent to the internal flow solver where forces and moments are evaluated and sent back to the seakeeping solver. For the exchange of data, the seakeeping solver has a TCL interface, allowing to execute TCL scripts that can access different internal data structures of the seakeeping solver during the calculation process. The internal flow solver includes a Python-based interface, developed to interchange data with external sources.

In order to implement the communication scheme, a TCL script [104] was built. This script is responsible for the communication between the two programs. It is interpreted and executed by the seakeeping solver and communicates with different instances of the internal flow solver. Figure 59 explains the communication scheme.

The exchange of data is carried out with respect to an instantaneous reference system. Table 28 and Table 29 show the data sent from the seakeeping solver to the internal flow solver and vice versa. 
The instantaneous reference system at time $t, R(t)$, is defined such that $R(0)$ coincides with the global reference system OXYZ. R(t) describes the trajectory of the point of the rigid body located initially at the origin of the global system, and its axes $\mathrm{X}_{\mathrm{t}} \mathrm{Y}_{\mathrm{t}} \mathrm{Z}_{\mathrm{t}}$ remain parallel to the global axes. To further understand the reference systems used, a simple rigid body movement is presented in Figure 60 showing the displacements and rotations.

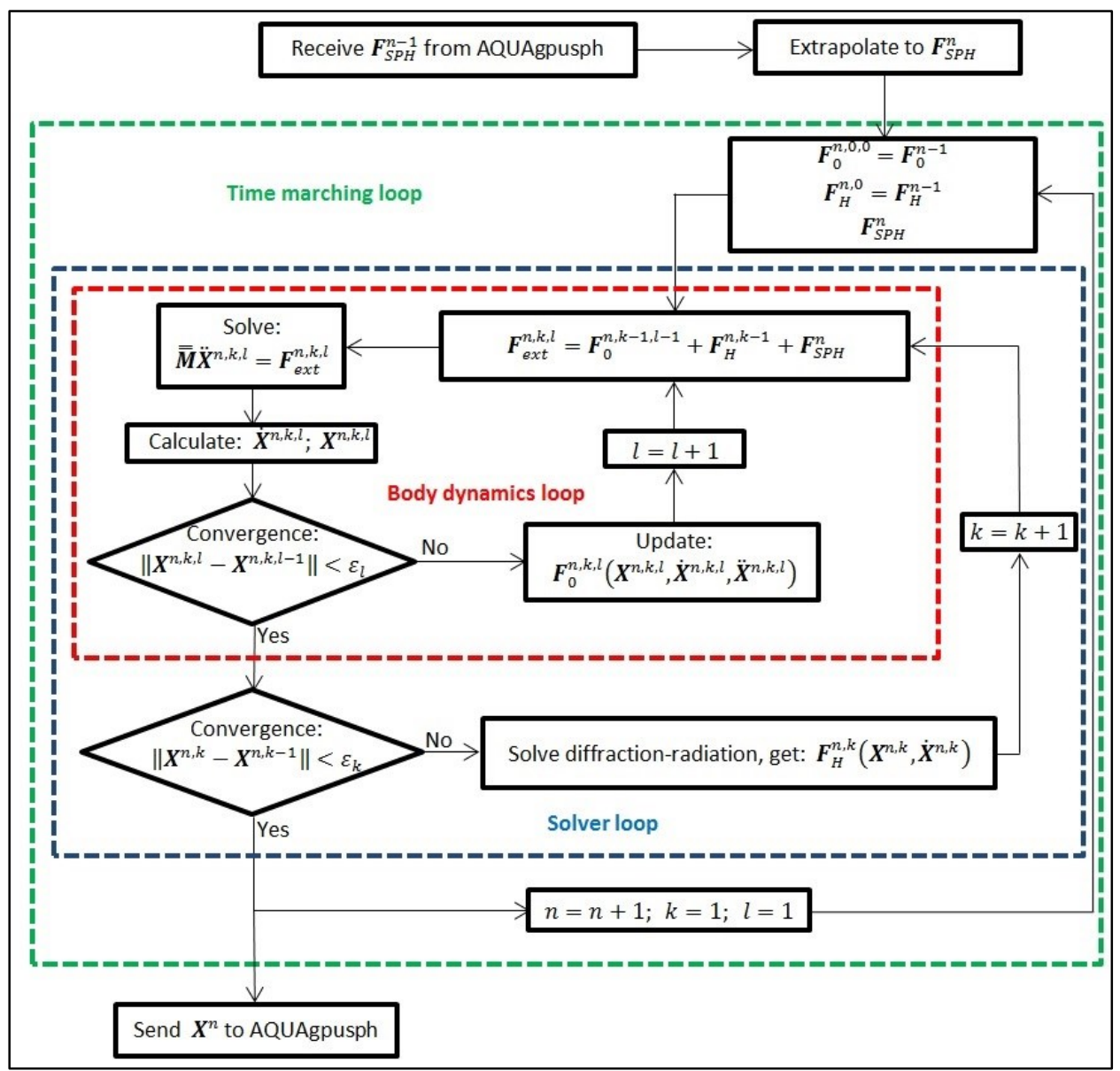

Figure 58: Body dynamics solver algorithm including loads from the internal flow solver.

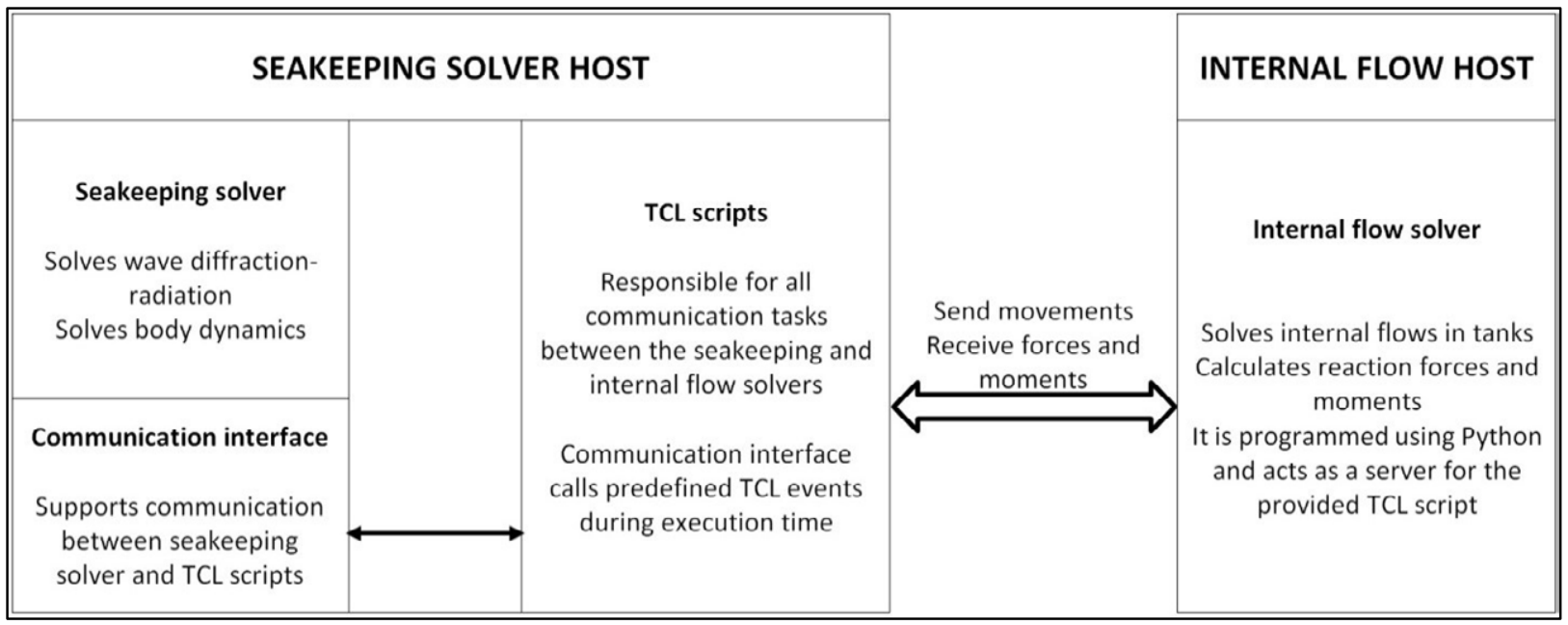

Figure 59: Communication scheme 
Table 28: data sent from the seakeeping solver to the internal flow solver

\begin{tabular}{|c|c|}
\hline $\mathrm{t}$ & Elapsed simulation time at each time-step \\
\hline $\mathrm{R}(\mathrm{t})=\left(\mathrm{X}_{\mathrm{R}}(\mathrm{t}), \mathrm{Y}_{\mathrm{R}}(\mathrm{t}) ; \mathrm{Z}_{\mathrm{R}}(\mathrm{t})\right)$ & $\begin{array}{l}\text { Coordinates of the body reference point R referred to the global } \\
\text { system OXYZ }\end{array}$ \\
\hline$\Theta(t)=\left(\Theta_{x}(t), \Theta_{y}(t) ; \Theta_{z}(t)\right)$ & Rotation Angles referred to the global system OXYZ of the body \\
\hline $\mathrm{V}_{\mathrm{R}}(\mathrm{t})=\left(\mathrm{V}_{\mathrm{x}}(\mathrm{t}), \mathrm{V}_{\mathrm{y}}(\mathrm{t}) ; \mathrm{V}_{\mathrm{z}}(\mathrm{t})\right)$ & $\begin{array}{l}\text { Linear velocities of the body reference point R referred to the global } \\
\text { system OXYZ }\end{array}$ \\
\hline$\omega(\mathrm{t})=\left(\omega_{\mathrm{x}}(\mathrm{t}), \omega_{\mathrm{y}}(\mathrm{t}) ; \omega_{\mathrm{z}}(\mathrm{t})\right)$ & Angular velocities of the body referred to the global system OXYZ \\
\hline
\end{tabular}

Table 29: data sent from the internal flow solver to the seakeeping solver

\begin{tabular}{|c|c|}
$F(t)=\left(F_{x}(t), F_{y}(t) ; F_{z}(t)\right)$ & Internal flow forces referred to the global system OXYZ \\
\hline$M(t)=\left(M_{x}(t), M_{y}(t) ; M_{z}(t)\right)$ & Internal flow moments referred to the global system OXYZ \\
\hline
\end{tabular}

\subsubsection{Coupling algorithm}

Since both solvers run in the time domain, the coupling is based on the exchange of data given in Table 28 and Table 29 at some specific time steps while both solvers are running. The time step required by the SPH solver $\left(\Delta \mathrm{t}_{\mathrm{SPH}}\right)$ stability criteria is usually in the order of 10 to 1000 times smaller than the time step used by the seakeeping solver $\left(\Delta \mathrm{t}_{\mathrm{FEM}}\right)$, depending on the case under study, the grid resolution and number of particles. Hence an explicit staggered approach was selected to couple both solvers. This scheme is summarized next:

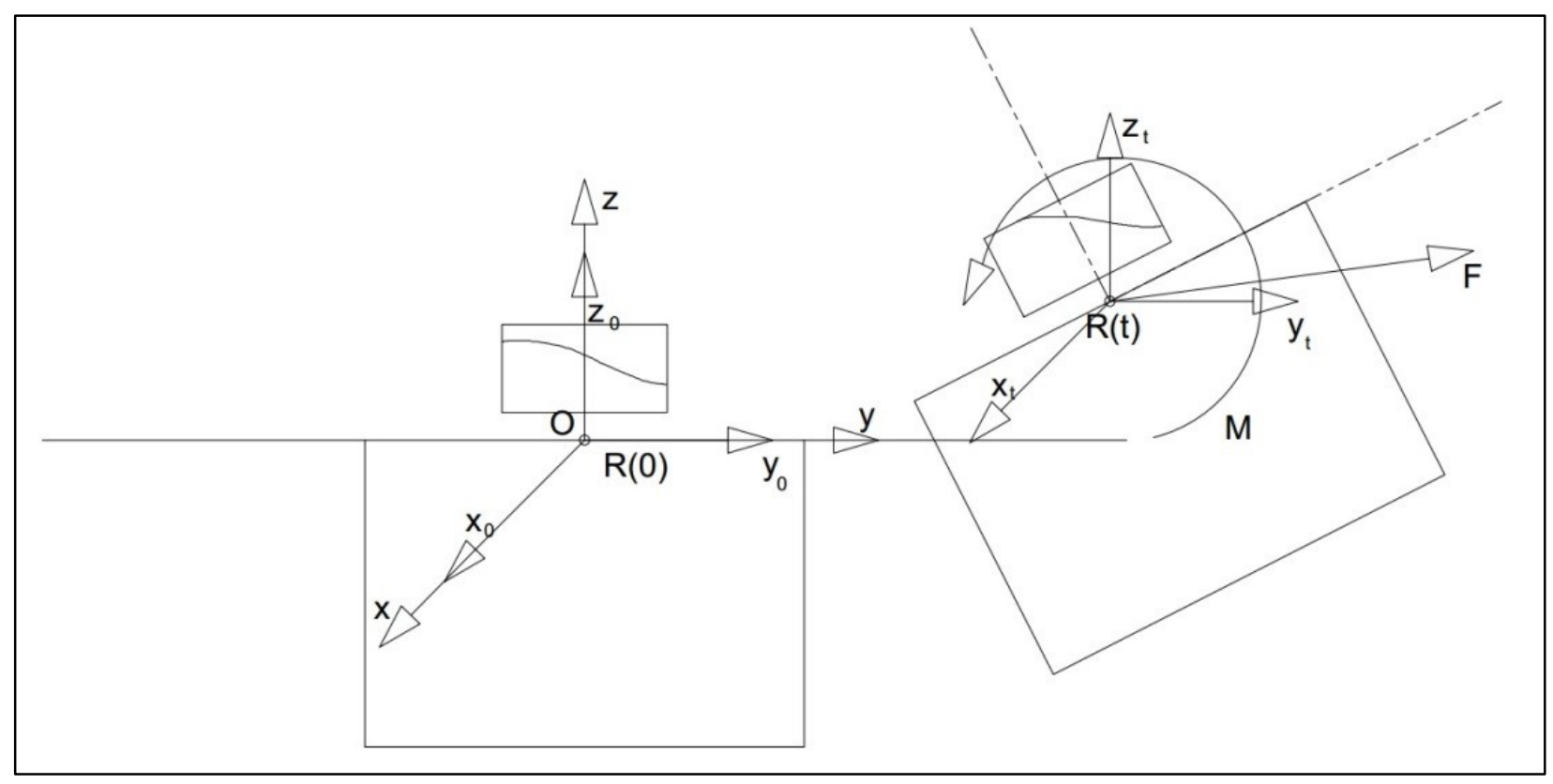

Figure 60: Rigid body movement and reference systems used. 
Forces and moments calculated by the internal flow solver at time $=n \Delta t_{F E M}$ are sent to the seakeeping solver.

1. The seakeeping solver extrapolates forces and moments from the internal flow solver at time $=$ $(n+1) \Delta t_{F E M}$ using a five points Lagrange polynomial.

2. The seakeeping solver calculates movements at time $=(n+1) \Delta t_{F E M}$.

3. The seakeeping solver sends movements to the internal flow solver at time $=(n+1) \Delta t_{F E M}$.

4. The internal flow solver runs from time $=n \Delta t_{F E M}$ to time $=(n+1) \Delta t_{F E M}$ interpolating the body movements sent by the seakeeping solver.

Figure 61 provides the details of the coupling algorithm implemented between the seakeeping and internal flow solvers.

\section{$10.5 \quad$ Validation}

\subsubsection{Barge with water in tanks}

\subsubsection{Case description}

In order to validate the present coupled internal flow-seakeeping solvers, experimental results obtained by [105] have been used to compare with. The experiments consist of the study of the seakeeping response of a barge-like ship, where a tank is extending over a relatively large part of the model. There are two tanks next to each other at the mid-ship whose transverse dimension is close to the model breadth (see Figure 62).

Figure 63 shows the unstructured mesh used in the seakeeping solver for the present case study. The mesh size in the floating line is $2.5 \mathrm{~cm}$, and a total of 236144 tetrahedral elements were used.

\subsubsection{Case 1: same water level in tanks}

The first case study to analyze is that where the water level is $19 \mathrm{~cm}$ in both tanks. To achieve the target draft of $10.8 \mathrm{~cm}$, additional mass of $40 \mathrm{~kg}$ was added on the deck of the barge. Table 30 provides the particulars of the barge including the additional mass. Only roll values were provided in Molin et al. [105]. Numerical simulations were carried out with free sway, heave and roll in order to better approximate the experiments. Since both tanks are filled with the same water level, and movements are occurring in the OYZ plane, only one tank was simulated and the second was assumed to behave equally.

Figure 64 represents the experimental results for the case study where both tanks are filled with the same water level $(19 \mathrm{~cm})$. The roll RAO in the experiments was obtained via spectral analysis from irregular motion tests. The wave spectrum used was a JONSWAP with particulars provided in Table 31. 


\begin{tabular}{|c|c|}
\hline SPH solver & FEM solver \\
\hline $\begin{array}{c}\text { Initialization: } \\
\text { Setup python TCP server. }\end{array}$ & $\begin{array}{l}\text { Initialization: } \\
\text { Establish connection with SPH server. }\end{array}$ \\
\hline $\begin{array}{c}\text { Calculate: } \\
\text { Static problem. }\end{array}$ & WAIT \\
\hline \multicolumn{2}{|c|}{ Start time iteration at: TIME $=0$} \\
\hline $\begin{array}{c}\text { SEND } \\
\mathbf{F}(\mathrm{t}=0) \quad(\text { weight of water in tank }) ; \mathbf{M}(\mathrm{t}=0)\end{array}$ & $\begin{array}{c}\text { RECEIVE } \\
\mathbf{F}(\mathrm{t}=0) ; \mathbf{M}(\mathrm{t}=0)\end{array}$ \\
\hline \multirow[b]{2}{*}{ WAIT } & $\begin{array}{c}\text { EXTRAPOLATE } \\
\text { Fe }\left(\mathrm{t}=\Delta t_{F E M}\right)=\mathbf{F}(\mathrm{t}=0) \\
\operatorname{Me}\left(\mathrm{t}=\Delta t_{F E M}\right)=\mathbf{M}(\mathrm{t}=0)\end{array}$ \\
\hline & $\begin{array}{c}\text { CALCULATE } \\
\text { FEM runs from } \mathrm{t}=0 \text { to } \mathrm{t}=\Delta \boldsymbol{t}_{F E M} \\
\mathbf{R}\left(\mathrm{t}=\Delta t_{F E M}\right), \boldsymbol{\Theta}\left(\mathrm{t}=\Delta t_{F E M}\right) ; \mathbf{V R}\left(\mathrm{t}=\Delta t_{F E M}\right) ; \mathbf{W}\left(\mathrm{t}=\Delta t_{F E M}\right)\end{array}$ \\
\hline $\begin{array}{c}\text { RECEIVE } \\
\text { time }=\Delta t_{S F} ; \mathbf{R}\left(\mathrm{t}=\Delta t_{F E M}\right), \boldsymbol{\Theta}\left(\mathrm{t}=\Delta t_{F E M}\right) ; \\
\mathbf{V}\left(\mathrm{t}=\Delta t_{F E M}\right) ; \mathbf{W}\left(\mathrm{t}=\Delta t_{F E M}\right)\end{array}$ & $\begin{array}{c}\text { SEND } \\
\text { time }=\Delta t_{S F} ; \mathbf{R}\left(\mathrm{t}=\Delta t_{F E M}\right), \boldsymbol{\Theta}(\mathrm{t}=\Delta F E M) \\
\mathbf{V R}\left(\mathrm{t}=\Delta t_{F E M}\right) ; \mathbf{W}\left(\mathrm{t}=\Delta t_{F E M}\right)\end{array}$ \\
\hline \multicolumn{2}{|r|}{ TIME $=\Delta t_{F E M}$} \\
\hline \multicolumn{2}{|c|}{$\ldots$} \\
\hline \multicolumn{2}{|c|}{ TIME $=\mathrm{i} \cdot \Delta t_{F E M}$} \\
\hline $\begin{array}{c}\text { CALCULATE } \\
\text { SPH runs from } \mathrm{t}=(\mathrm{i}-1) \cdot \Delta t_{F E M} \text { to } \mathrm{t}=\mathrm{i} \cdot \Delta t_{F E M}\end{array}$ & WAIT \\
\hline $\begin{array}{c}\text { SEND } \\
\mathbf{F}\left(\mathrm{t}=\mathrm{i} \cdot \Delta t_{F E M}\right), \mathbf{M}\left(\mathrm{t}=\mathrm{i} \cdot \Delta t_{F E M}\right)\end{array}$ & $\begin{array}{c}\text { RECEIVE: } \\
\mathbf{F}\left(\mathrm{t}=\mathrm{i} \cdot \Delta t_{F E M}\right), \mathbf{M}\left(\mathrm{t}=\mathrm{i} \cdot \Delta t_{F E M}\right)\end{array}$ \\
\hline \multirow{2}{*}{ WAIT } & $\begin{array}{c}\text { EXTRAPOLATE } \\
\mathbf{F e}\left(\mathrm{t}=(\mathrm{i}+1) \Delta t_{F E M}\right), \mathbf{M e}\left(\mathrm{t}=(\mathrm{i}+1) \Delta t_{F E M}\right) \text { as: } \\
\mathbf{F e}\left((\mathrm{i}+1) \cdot \Delta t_{F E M}\right)=\mathrm{f}\left(\mathbf{F}\left(\mathrm{i} \cdot \Delta t_{F E M}\right), \mathbf{F}\left((\mathrm{i}-1) \cdot \Delta t_{F E M}\right), \ldots\right) \\
\mathbf{M e}\left((\mathrm{i}+1) \cdot \Delta t_{F E M}\right)=\mathrm{f}\left(\mathbf{M}\left(\mathrm{i} \cdot \Delta t_{F E M}\right),\right. \\
\left.\mathbf{M}\left((\mathrm{i}-1) \cdot \Delta t_{F E M}\right), \ldots\right)\end{array}$ \\
\hline & $\begin{array}{c}\text { CALCULATE } \\
\text { FEM runs from } \mathrm{t}=\left(\mathrm{i} \cdot \Delta t_{F E M}\right) \text { to } \mathrm{t}=(\mathrm{i}+1) \cdot \Delta t_{F E M} \\
\mathbf{R}\left(\mathrm{t}=(\mathrm{i}+1) \cdot \Delta t_{F E M}\right), \mathbf{\Theta}\left(\mathrm{t}=(\mathrm{i}+1) \cdot \Delta t_{F E M}\right) \\
\left.\mathbf{V R}\left(\mathrm{t}=(\mathrm{i}+1) \cdot \Delta t_{F E M}\right) ; \mathbf{W}(\mathrm{t}=(\mathrm{i}+1) \cdot) \cdot \Delta t_{F E M}\right)\end{array}$ \\
\hline $\begin{array}{c}\mathbf{R E C E I V E} \\
\text { time }=\mathrm{i} \cdot \Delta t_{F E M} \\
\mathbf{R}\left(\mathrm{t}=\mathrm{i} \cdot \Delta t_{F E M}\right), \boldsymbol{\Theta}\left(\mathrm{t}=\mathrm{i} \cdot \Delta t_{F E M}\right) \\
\mathbf{V}\left(\mathrm{t}=\mathrm{i} \cdot \Delta t_{F E M}\right) ; \mathbf{W}\left(\mathrm{t}=\mathrm{i} \cdot \Delta t_{F E M}\right)\end{array}$ & $\begin{array}{c}\text { SEND } \\
\text { time }=\mathrm{i} \cdot \Delta t_{F E M} \\
\mathbf{R}\left(\mathrm{t}=\mathrm{i} \cdot \Delta t_{F E M}\right), \boldsymbol{\Theta}\left(\mathrm{t}=\mathrm{i} \cdot \Delta t_{F E M}\right) \\
\mathbf{V R}\left(\mathrm{t}=\mathrm{i} \cdot \Delta t_{F E M}\right) ; \mathbf{W}\left(\mathrm{t}=\mathrm{i} \cdot \Delta t_{F E M}\right)\end{array}$ \\
\hline
\end{tabular}

Figure 61: Internal flow-seakeeping coupling algorithm 


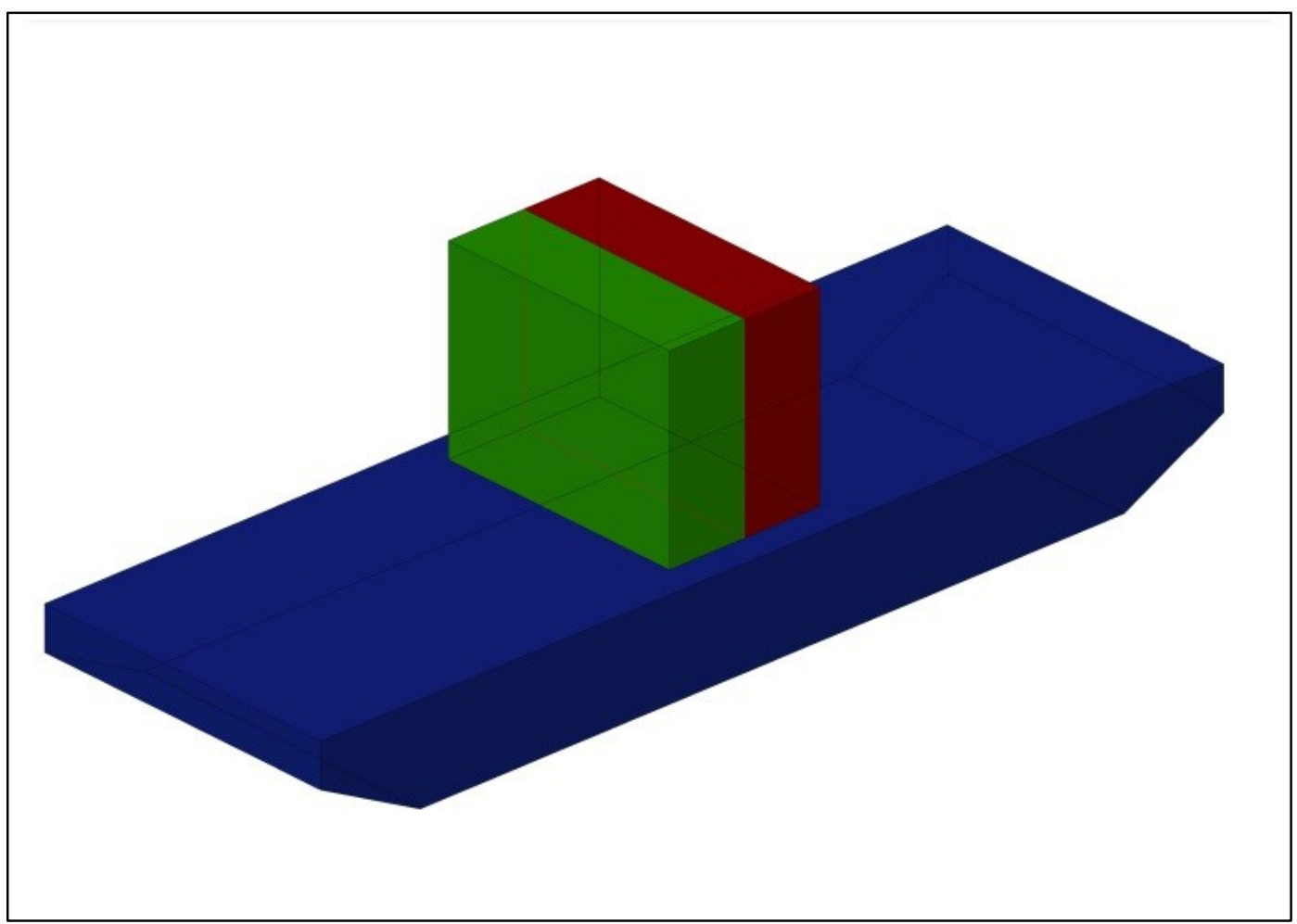

Figure 62: Barge with tanks.

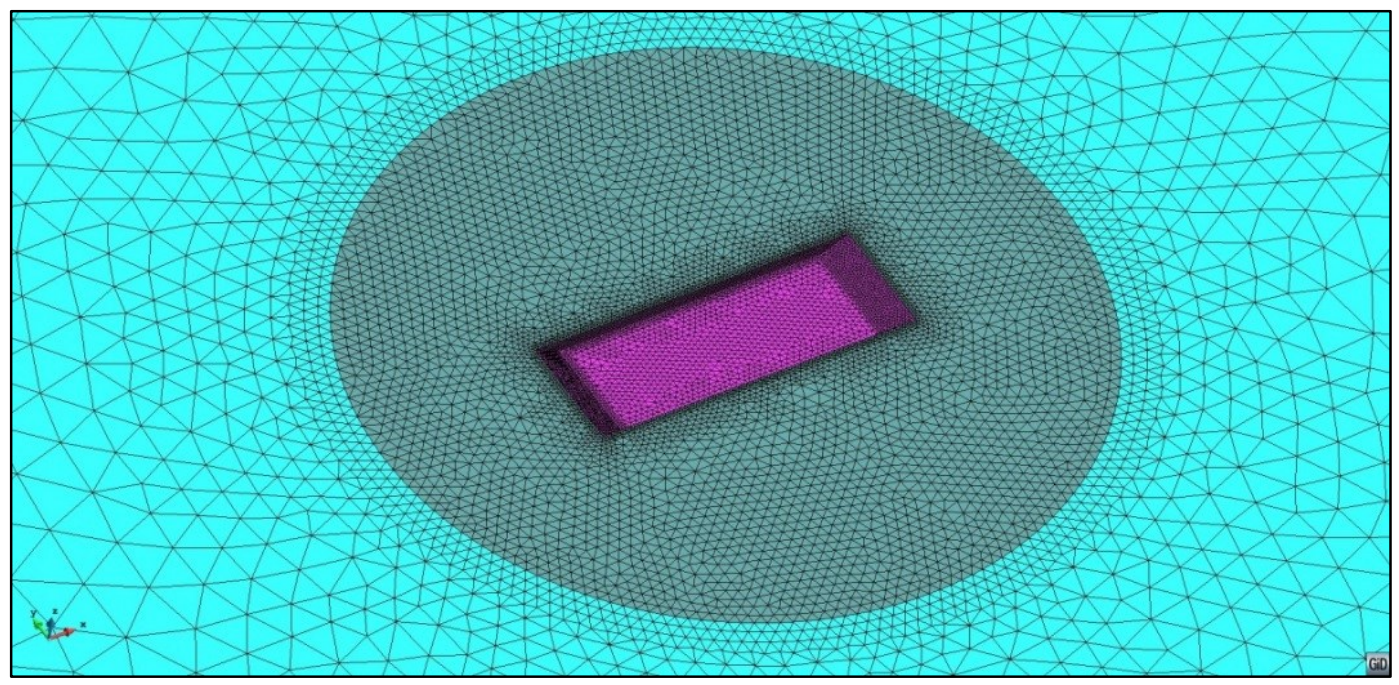

Figure 63: FEM mesh.

Table 30: Barge main particulars including the additional mass of 40kg on deck.

\begin{tabular}{|c|c|}
\hline Length & $3 \mathrm{~m}$ \\
\hline Breath & $1 \mathrm{~m}$ \\
\hline Target draft & $0.108 \mathrm{~m}$ \\
\hline Displacement & $275 \mathrm{~kg}$ \\
\hline XG & $0 \mathrm{~m}$ \\
\hline YG & $0 \mathrm{~m}$ \\
\hline ZG & $0.14 \mathrm{~m}$ \\
\hline Radii of gyration respect to G: Rxx & $0.3704 \mathrm{~m}$ \\
\hline
\end{tabular}


Table 31: Jonswap spectrum used in experiments.

\begin{tabular}{|c|c|}
\hline Peak enhancement factor $\boldsymbol{\gamma}$ & 2 \\
\hline Significant wave height $\mathbf{H}_{\mathbf{S}}$ & $6.6 \mathrm{~cm}$ \\
\hline Peak period $\mathbf{T}_{\mathbf{p}}$ & $1.6 \mathrm{~s}$ \\
\hline
\end{tabular}

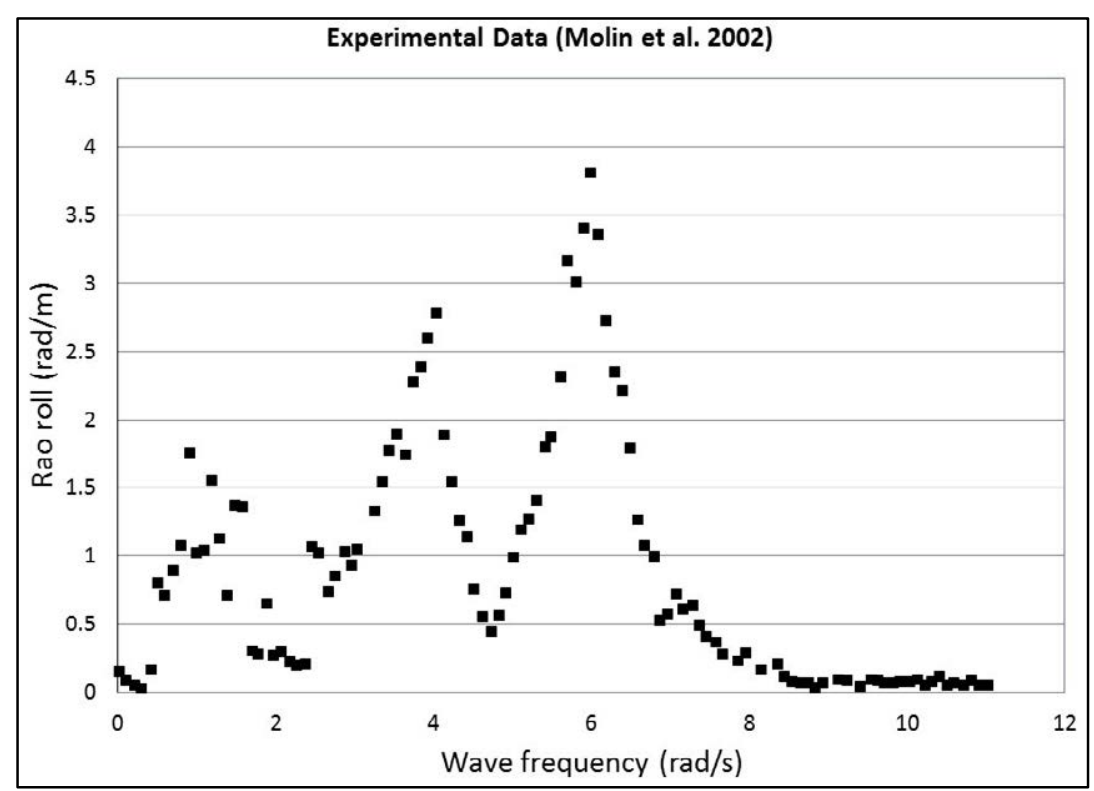

Figure 64: Experimental results obtained in Molin et al. [105].

\section{Monochromatic wave test}

First, a calibration of the roll damping to account for viscous effects is carried out. A monochromatic test with wave amplitude of $2 \mathrm{~cm}$ and wave frequency $\omega=4.026 \mathrm{rad} / \mathrm{s}$, which is where the first resonance appears, was used. The test was carried out with $10^{5}$ particles and it was found that a roll damping factor of $\mathrm{C}=0.11 \sqrt{4 \mathrm{I}_{\mathrm{xx}} \mathrm{K}_{44}}$ was necessary to reproduce the RAO value of the experiments. Ludvigsen et al. [106] reported a value of $8 \%$ of the critical damping.

Second, several tests were carried out to check the effect of increasing the number of particles in the SPH solver. It was selected a wave frequency $\omega=5.984 \mathrm{rad} / \mathrm{s}$, which corresponds to the second resonance frequency in Figure 64. All cases were simulated including a damping factor of $\mathrm{C}=$ $0.11 \sqrt{4 \mathrm{I}_{\mathrm{xx}} \mathrm{K}_{44}}$, where $\mathrm{I}_{\mathrm{xx}}$ is the roll inertia of the system barge plus weights, and $\mathrm{K}_{44}$ is the roll hydrostatic restoring coefficient.

Table 32 provides the roll amplitude obtained for each case. The amplification factor converges, slowly, to a value about 2.7 .

Table 32: Roll amplitude in $\mathrm{rad} / \mathrm{m}$ for monochromatic test cases.

\begin{tabular}{|c|c|}
\hline Number of particles & RAO Roll [rad/m] \\
\hline $\mathbf{1 0 , 0 0 0}$ & 2.29 \\
\hline $\mathbf{3 5 , 0 0 0}$ & 2.45 \\
\hline $\mathbf{1 0 0 , 0 0 0}$ & 2.55 \\
\hline $\mathbf{2 5 0 , 0 0 0}$ & 2.60 \\
\hline $\mathbf{5 0 0 , 0 0 0}$ & 2.64 \\
\hline
\end{tabular}




\section{Irregular wave test}

Next a RAO analysis test is carried out. The irregular waves used in the numerical simulation correspond to a JONSWAP spectrum with similar characteristics of that used in the experiments (see Table 31). The discretization of the wave spectrum was done using 51 frequencies from 2.5 to 8 radians per second. Also a roll damping $\mathrm{C}=0.11 \sqrt{4 \mathrm{I}_{\mathrm{xx}} \mathrm{K}_{44}}$ was used.

Figure 65 compares the results obtained in the present work using $10^{5}$ particles against those from experiments and calculated by WADAM in Ludvigsen et al. [106] using a potential flow approach to account for the fluid dynamics within the tank. It can be observed that while WADAM provides a smooth curve of results, experiments and the present work show irregularities. This might be because the SPH approach for the fluid flow in the tank is nonlinear, which contributes to energy transfer among frequencies.

For frequencies around $\omega=6 \mathrm{rad} / \mathrm{s}$, experiments show larger values than those obtained in the computational analyses. On the other hand, results obtained in this work are a bit larger than those obtained by WADAM, and they both compare well with the experiment for frequencies away from $\omega=6 \mathrm{rad} / \mathrm{s}$.

\section{Computational time}

Table 33 provides some data regarding the computational effort needed for the present approach. The computational time corresponds to those cases presented in the monochromatic wave test. It is observed that the calculation of the fluid flow within the tank using the SPH with more than $3.5 \cdot 10^{4}$ particles is much more expensive than calculating the seakeeping behavior. Furthermore, if the number of particles is bumped up to $5 \cdot 10^{5}$ then this solver takes more than $98 \%$ of the calculation time.

\subsubsection{Case 2: different water level in tanks}

The second case study is the case where the water level in one tank is $19 \mathrm{~cm}$ and in the other 39 $\mathrm{cm}$. No additional mass is necessary to achieve the target draft like in the previous case study. The wave amplitude distribution used to mimic the irregular wave conditions of the experiments is provided in the appendix. The discretization of the wave spectrum was done using one 101 frequencies ranging from 2.5 to $8 \mathrm{rad} / \mathrm{s}$. Also a roll damping $\mathrm{C}=0.11 \sqrt{4 \mathrm{I}_{\mathrm{xx}} \mathrm{K}_{44}}$ was used. The main particulars of the barge for this case study are given in Table 34 .

Figure 66 compares the results obtained in the present work against those from experiments and calculated by WADAM in Ludvigsen et al. [106]. One hundred thousand particles were used in the 19 $\mathrm{cm}$ filling tank, and $2 \cdot 10^{5}$ particles in the $39 \mathrm{~cm}$ filling tank. In this case study, three resonance peaks appear. For the first peak, with frequencies around $\omega=4 \mathrm{rad} / \mathrm{s}$, the largest deviation from numerical to experimental results is found. The second peak, with frequencies that are around $\omega=5.25 \mathrm{rad} / \mathrm{s}$ 
no apparent deviation of the frequency of the peaks is observed and the numerical results around the sharp peak achieve a very similar value to the experimental results. Finally, for frequencies around $\omega=$ $7 \mathrm{rad} / \mathrm{s}$, no deviation in frequency is observed, but numerical results are below the predicted peak value.

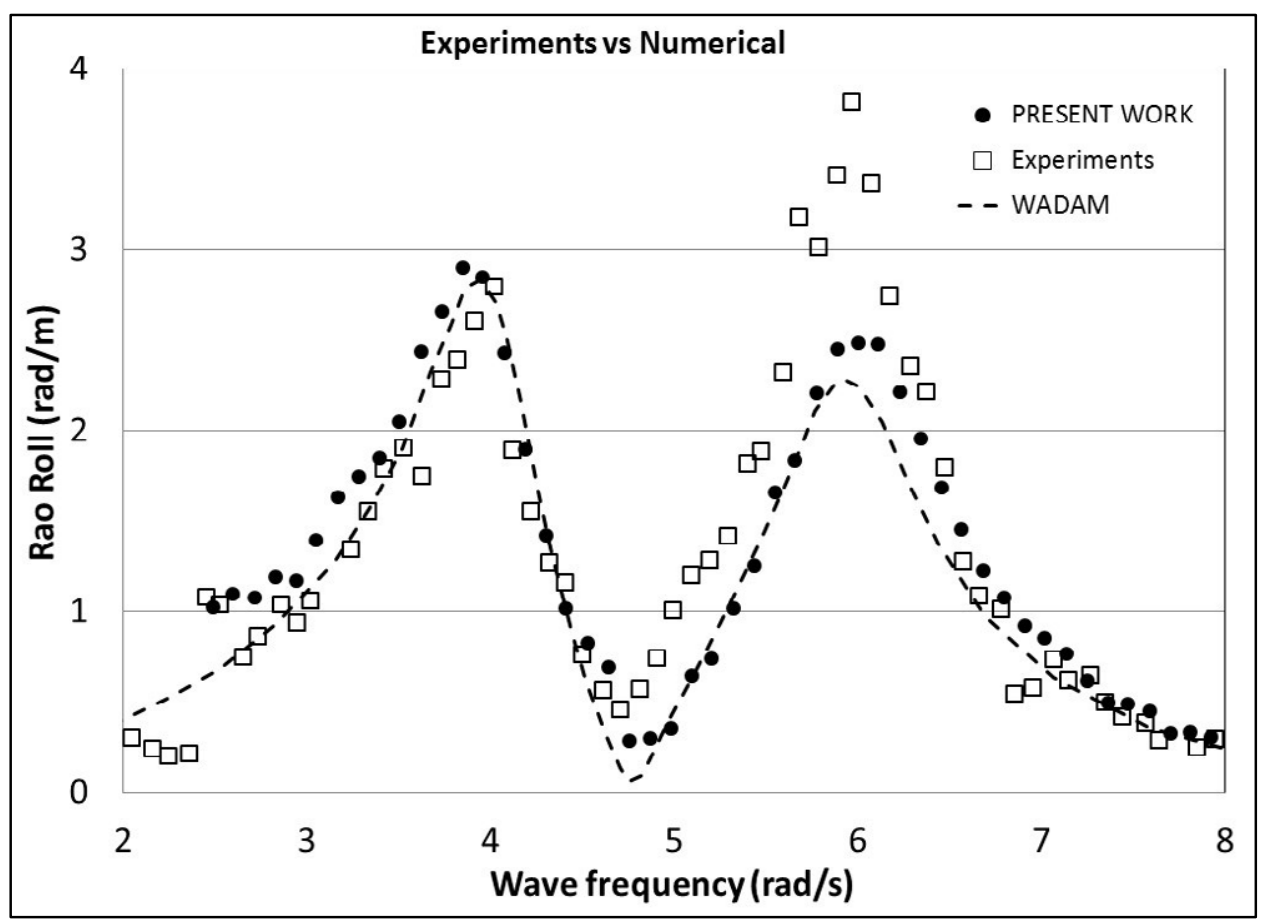

Figure 65: Results comparison among FEM-SPH coupling (present work), WADAM Ludvigsen et al. [106], and experiments Molin et al. [105].

Table 33: Computational time

\begin{tabular}{|c|c|c|c|c|c|}
\hline $\begin{array}{c}\text { Number } \\
\text { of particles }\end{array}$ & $\mathbf{\Delta} \mathbf{t}_{\mathbf{S F}}(\mathbf{s})$ & $\begin{array}{c}\text { Simulation } \\
\text { time (s) }\end{array}$ & $\begin{array}{c}\text { Computational } \\
\text { time (s) }\end{array}$ & $\begin{array}{c}\text { SPH } \\
(\mathbf{\% )}\end{array}$ & $\begin{array}{c}\text { FEM } \\
(\mathbf{\% )}\end{array}$ \\
\hline $\mathbf{1 0 , 0 0 0}$ & 0.01 & 30 & 1938 & 33.5 & 66.5 \\
\hline $\mathbf{3 5 , 0 0 0}$ & 0.01 & 30 & 4109 & 68.4 & 31.6 \\
\hline $\mathbf{1 0 0 , 0 0 0}$ & 0.01 & 30 & 13110 & 88.5 & 11.5 \\
\hline $\mathbf{2 5 0 , 0 0 0}$ & 0.01 & 30 & 41577 & 95.5 & 4.5 \\
\hline $\mathbf{5 0 0 , 0 0 0}$ & 0.01 & 30 & 98616 & 98.2 & 1.8 \\
\hline
\end{tabular}

Table 34: Barge main particulars with no additional mass on deck.

\begin{tabular}{|c|c|}
\hline Target draft & $0.108 \mathrm{~m}$ \\
\hline Displacement & $275 \mathrm{~kg}$ \\
\hline XG & $0 \mathrm{~m}$ \\
\hline YG & $0 \mathrm{~m}$ \\
\hline ZG & $0.132 \mathrm{~m}$ \\
\hline Radii of gyration respect to G: Rxx & $0.414 \mathrm{~m}$ \\
\hline
\end{tabular}




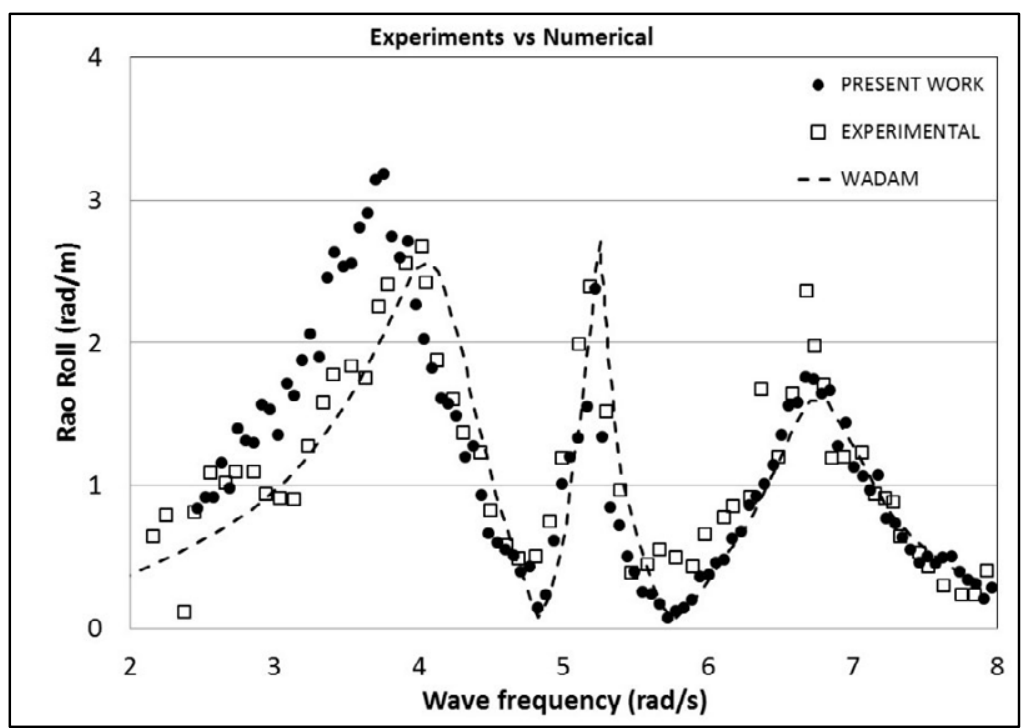

Figure 66: Results comparison among FEM-SPH coupling (present work), WADAM Ludvigsen et al. [106], and experiments Molin et al. [105].

\subsubsection{Antiroll tank analysis}

Bai and Rhee [107] provided experimental RAOs, obtained in a model basin, for a supply vessel equipped with an anti-roll tank (ART). Later on, Kim et. al $[108,85]$ carried out numerical simulations of a modified S175 hull in order to compare the results with the experimental data provided by Bai and Rhee [107]. The modified S175 hull used by Kim et. al was an approximation to the supply vessel used by Bai and Rhee and results obtained by Kim et. al numerically showed the same trends than the experimental ones.

In this work, Kim et. al approach, using a modified S175 hull to approximate the supply one used by Bai and Rhee, has been followed. The same aspect ratios of beam to length, and draft to length used by Kim [85], for the modified S175 hull and the ART, have been considered. However, since no information regarding the exact geometry was found, neither for the position of the gravity center nor for the inertias. Thus some parameters as well as the hull form have been estimated.

The S175 hull form provided by the ITTC web site was taken as a first estimate for the hull form. Then, it was proceeded to scale the ship to fulfill the aspect ratios provided by Kim et. al in [85]. The resulting geometry is provided in [109]. The longitudinal positions of the gravity center and ART were estimated as the longitudinal position of the buoyancy center. The transverse positions was set to $\mathrm{Y}=$ $0 \mathrm{~m}$, and the vertical position of the gravity center and roll radii of gyration were estimated to approximate roll RAOS results without ART provided by Kim et. al. [85]. Table 35 and Table 36 provide the main particulars of the hull form and ART used in this work. Figure 67 shows the modified S175 hull with the ART in place. 
Roll RAOs were obtained for a series of tests with monochromatic waves and were normalized with respect to the maximum wave slope $\mathrm{A} \cdot \mathrm{k}$, where $\mathrm{A}$ is the wave amplitude, and $\mathrm{k}$ the wave number. Also a damping factor of $\mathrm{C}=0.04 \sqrt{4 \mathrm{I}_{\mathrm{xx}} \mathrm{K}_{44}}$ was used to calibrate the $\mathrm{RAO}$ value near resonance.

Figure 68 compares the experimental results obtained by Bai and Rhee [107] with the numerical results obtained in this work where the results are shown with and without ART. Ten thousand particles were used in the SPH solver for the ART SPH simulation. The modified S175 was calibrated to show a behaviour similar to the supply vessel of Bai and Rhee without ART, as can be observed by comparing the RAOs without ART. When inserting the ART the results tend to be the same to the ones obtained by Bai and Rhee with a very similar reduction of roll movements.

Figure 69 presents some snapshots of the modified S175 hull with the ART at the resonance wave frequency. It can be observed that the roll motion is very small, and the fluid flow inside the ART is very nonlinear, exhibiting wave breaking phenomena.

Table 35: Modified S175 main particulars without ART
\begin{tabular}{|c|c|}
\hline Length between perpendiculars $(L)$ & $47.6 \mathrm{~m}$ \\
\hline Breadth & $13.7088 \mathrm{~m}$ \\
\hline Draft & $3.9984 \mathrm{~m}$ \\
\hline Displacement & $1498.4 \mathrm{~T}$ \\
\hline $\mathbf{X}_{\mathbf{F P}}$ & $23.8 \mathrm{~m}$ \\
\hline $\mathbf{X}_{\text {AP }}$ & -23.8 \\
\hline XG & $-0.73 \mathrm{~m}$ \\
\hline YG & 0 \\
\hline ZG & $-0.25 \mathrm{~m}$ \\
\hline Radius of roll gyration Rxx & $6.26 \mathrm{~m}$ \\
\hline
\end{tabular}

Table 36: Particulars of the ART

\begin{tabular}{|c|c|}
\hline Length & $2.8 \mathrm{~m}$ \\
\hline Breadth & $13.699 \mathrm{~m}$ \\
\hline Draft & $2.4 \mathrm{~m}$ \\
\hline Filling & $50 \%$ \\
\hline XT (mid tank) & $-0.73 \mathrm{~m}$ \\
\hline YT (mid tank) & $0 \mathrm{~m}$ \\
\hline ZT (base tank) & $-1.8564 \mathrm{~m}$ \\
\hline
\end{tabular}




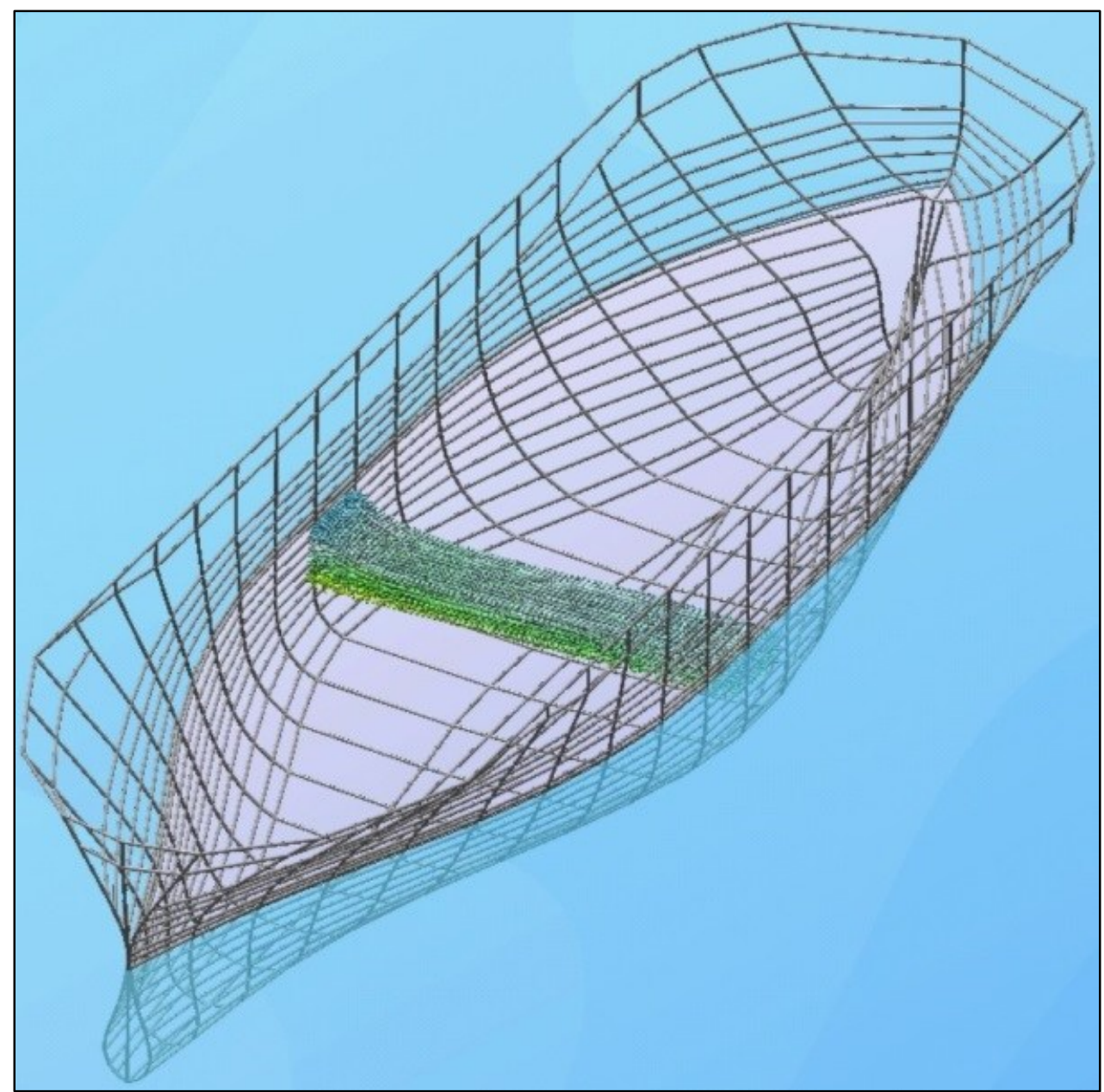

Figure 67: Modified S175 hull with ART.

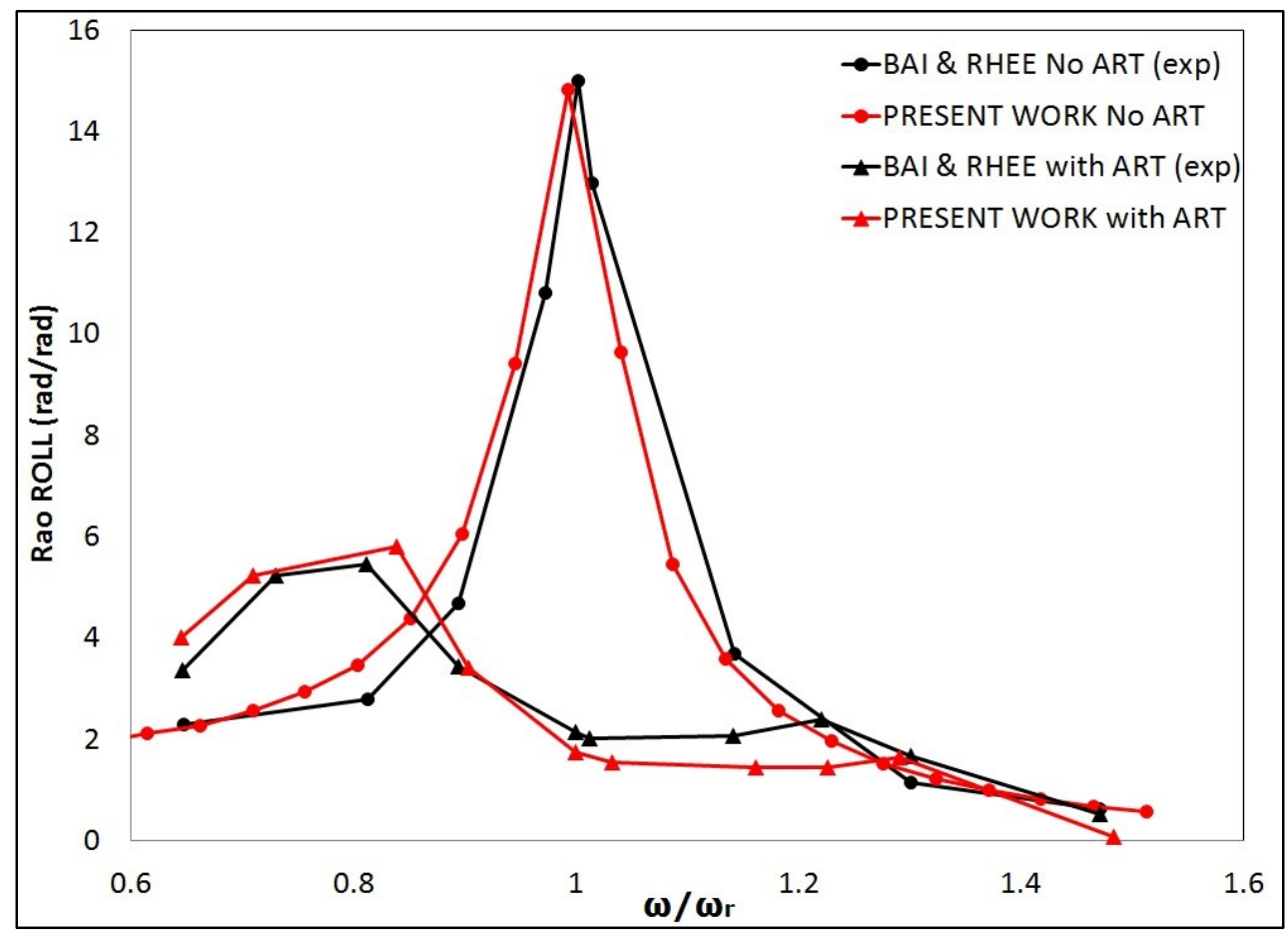

Figure 68: Experimental vs present work. Roll RAOs for monochromatic wave tests with $\mathrm{A} / \mathrm{L}=0.005$. Roll RAOs are obtained with maximum wave slope AK. 


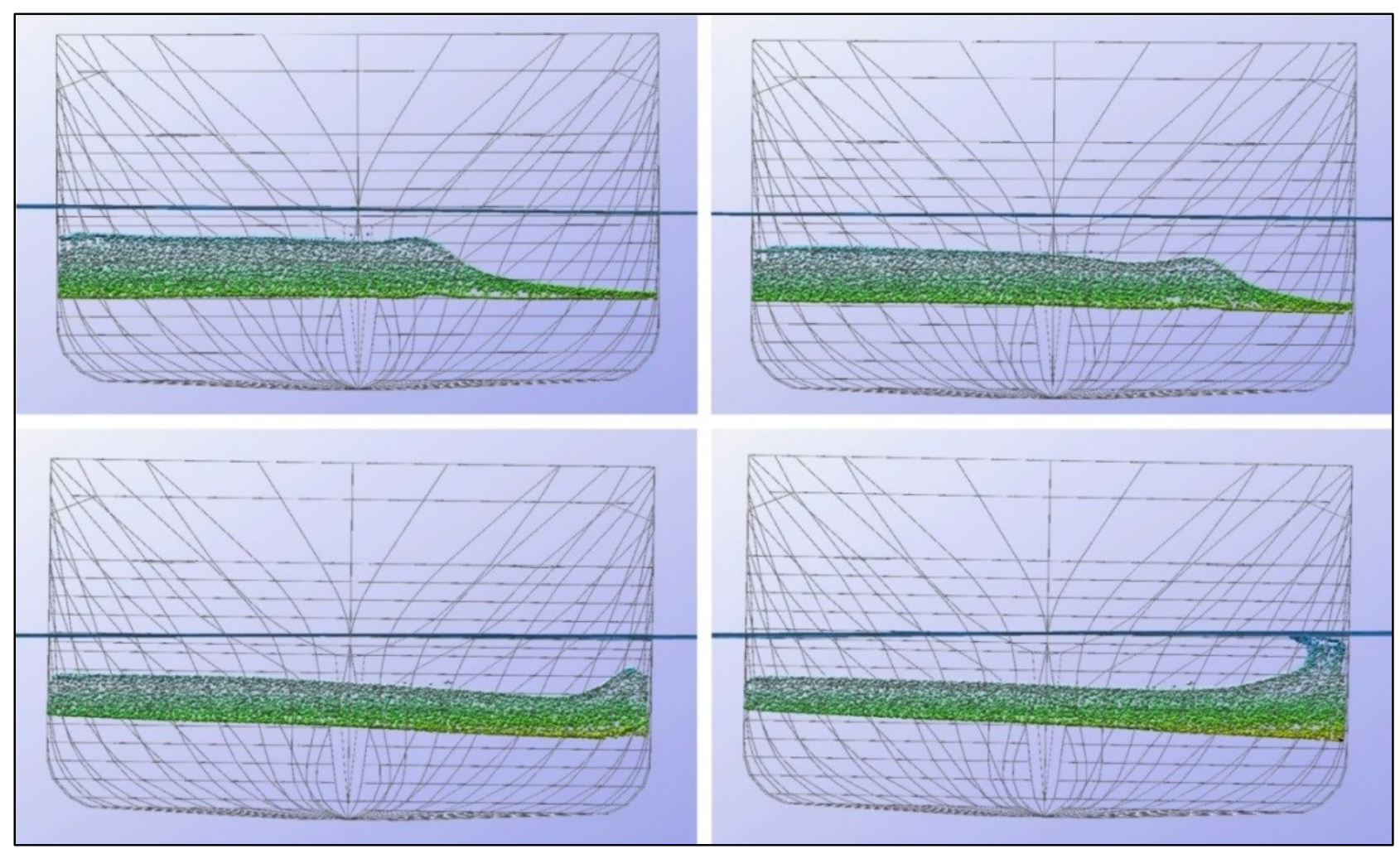

Figure 69: Snapshots of modified S175 with SRT for $\omega \sqrt{\mathrm{L} / \mathrm{g}}=1.55$ and $\mathrm{A} / \mathrm{L}=0.005$.

\subsubsection{D vessel including internal flows}

Zhao et al. [110] carried out a series of two-dimensional model tests to study the hydrodynamic performance of a FLNG section including internal flow oscillations. The FLNG section was ballasted with fresh water and equivalent solid weights respectively, to clarify the coupling effects. RAOs of both motion responses and internal sloshing flows were calculated based on measured data. Particulars of the vessel section, internal tank, and complete model are provided in Table 37-Table 39, and Figure 70 shows the 3D FEM mesh model used for the simulation.

A white noise spectrum, with an energy density approximated to the one used in the experiments, was used to carry out the simulations. It consists of 51 frequencies equally spaced between $\omega_{\min }=$ $0.3918 \mathrm{rad} / \mathrm{s}$ and $\omega_{\max }=16.6165 \mathrm{rad} / \mathrm{s}$, with a wave amplitude of $\mathrm{A}=0.14 \mathrm{~m}$. First of all, uncoupled simulations with the equivalent solid weight were carried out to calibrate damping coefficients. Comparing with the solid weight experimental results (see Figure 71 left), damping coefficients were estimated as $\mathrm{C}_{33}=0.2 \sqrt{4 \mathrm{MK}_{33}}$ for heave, and $\mathrm{C}_{44}=0.055 \sqrt{4 \mathrm{I}_{\mathrm{xx}} \mathrm{K}_{44}}$ for roll. Secondly, coupled simulations were carried out using around $10^{4}$ particles within the tank. RAOs comparisons are provided in Figure 71 (right).

Overall, the results obtained in this thesis fit well the experimental results provided by Zhao et al. [110]. Some differences appear regarding the resonance frequency in roll, which might be due to the experimental setup to enforce the $2 \mathrm{D}$ condition, which is not fully simulated in the computational 
model. Besides, the numerical results for the coupled problem show a peak value in frequencies around the uncoupled resonance frequency with $\omega=\sim 0.6 \mathrm{rad} / \mathrm{s}$. Although this effect is not present in the experimental results, it was observed in the previous case study with the ART (see Figure 68). Considering this, the appearance of this peak is not surprising, and whether it should appear or not is questionable.

\subsection{Summary}

In this chapter, a SPH flow solver and the FEM seakeeping solver were coupled to perform coupled simulations of seakeeping dynamics and internal flows in tanks simultaneously. Since both solvers run independently in the time-domain, a coupling algorithm, including a communication strategy, was developed.

The SPH-FEM coupling showed to be effective for solving coupled seakeeping dynamics with internal flows including sloshing. It was validated for several cases against available experimental data, providing good agreement. For the specific case study of the modified S175 hull with ART, where sloshing effects were observed, good agreement was found, demonstrating the capability of incorporating highly non-linear phenomena. As a result, the proposed coupling strategy has the capability of performing complex seakeeping analysis coupled with highly non-linear internal flows in reasonable computational times.

Table 37: Particulars of the internal tank (test case [110]).

\begin{tabular}{|c|c|c|}
\hline Tank & Full scale & model scale \\
\hline Length $(\mathbf{m})$ & 40 & 0.8 \\
\hline Breadth $(\mathbf{m})$ & 10 & 0.2 \\
\hline Water level $(\mathbf{m})$ & 18 & 0.36 \\
\hline Mass $(\mathbf{k g})$ & $7.2 \mathrm{E}+06$ & 57.6 \\
\hline ZG $(\mathbf{m})$ & -4.72 & -0.0944 \\
\hline Inertia respect to tank CG $\mathbf{~ x x}\left(\mathbf{k g ~ m}^{\mathbf{2}}\right)$ & $1.15 \mathrm{E}+09$ & 3.69 \\
\hline Radius of roll gyration $\mathbf{R x x}(\mathbf{m})$ & 12.7 & 0.25 \\
\hline
\end{tabular}

Table 38: Particulars of the vessel section (test case [110]).

\begin{tabular}{|c|c|c|}
\hline Section & Full scale & Model scale \\
\hline Length $(\mathbf{m})$ & 50 & 1 \\
\hline Breadth $(\mathbf{m})$ & 17.5 & 0.35 \\
\hline Mass $(\mathbf{k g})$ & $4.805 \mathrm{E}+06$ & 38 \\
\hline ZG $(\mathbf{m})$ & -1.0473 & -0.021 \\
\hline Inertia respect to section CG Ixx $\left(\mathbf{k g ~ m}^{\mathbf{2}}\right)$ & $1.027 \mathrm{E}+09$ & 3.287 \\
\hline Radius of roll gyration Rxx $\mathbf{( m )}$ & 14.621 & 0.2924 \\
\hline
\end{tabular}


Table 39: Particulars of the complete model (test case [110]).

\begin{tabular}{|c|c|c|}
\hline Complete model & Full scale & model scale \\
\hline Draft $(\mathbf{m})$ & 13.72 & 0.2744 \\
\hline Mass $(\mathbf{k g})$ & $1.20 \mathrm{E}+07$ & 96.04 \\
\hline ZG $(\mathbf{m})$ & -3.25 & -0.065 \\
\hline Inertia respect to complete model CG Ixx $\left.\mathbf{( k g ~ m}^{\mathbf{2}}\right)$ & $2.22 \mathrm{E}+09$ & 7.1054 \\
\hline Radius of roll gyration Rxx $(\mathbf{m})$ & 13.6 & 0.272 \\
\hline
\end{tabular}

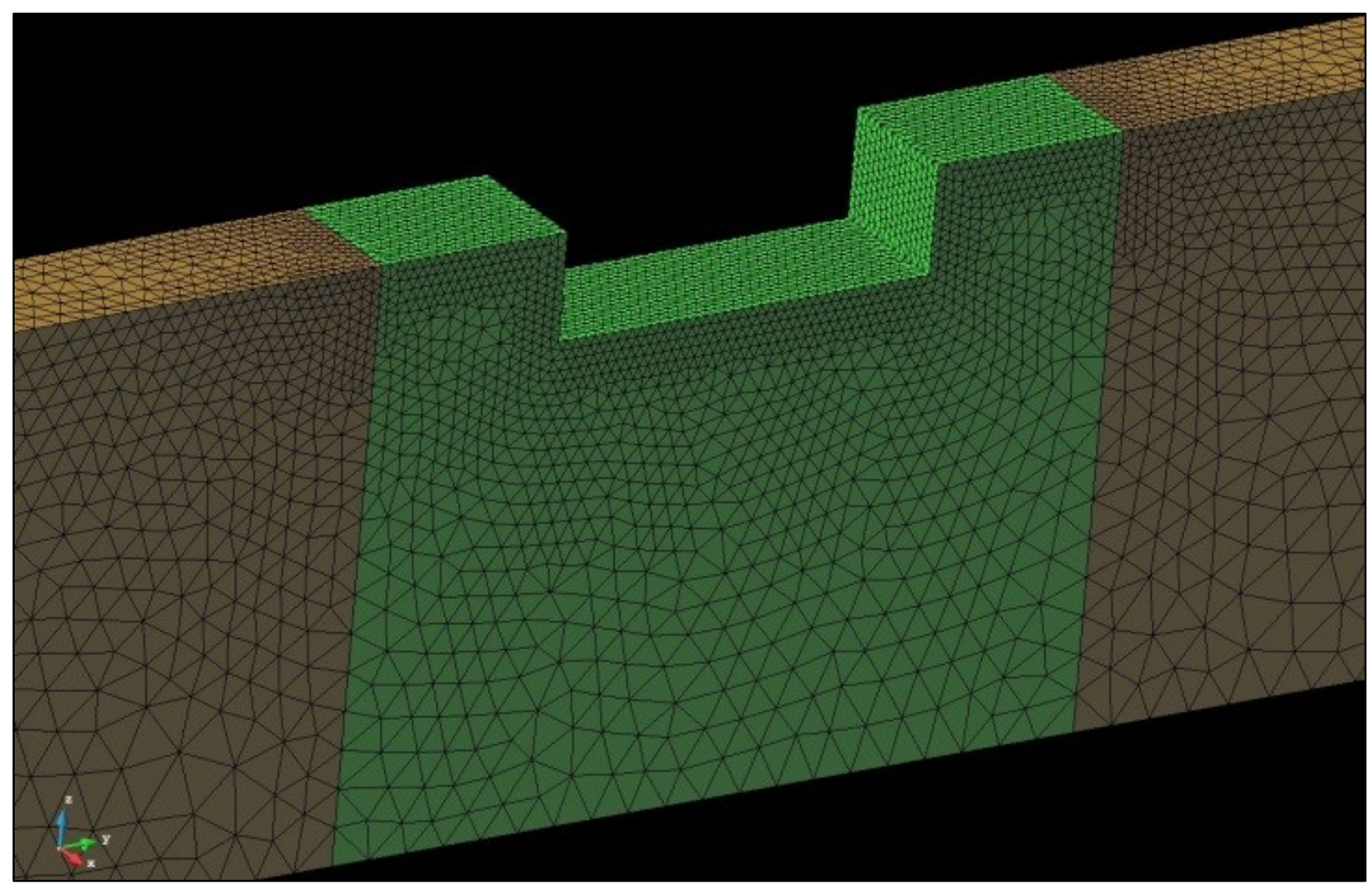

Figure 70: FEM -3D mesh for 2D cross section (test case [110]). 


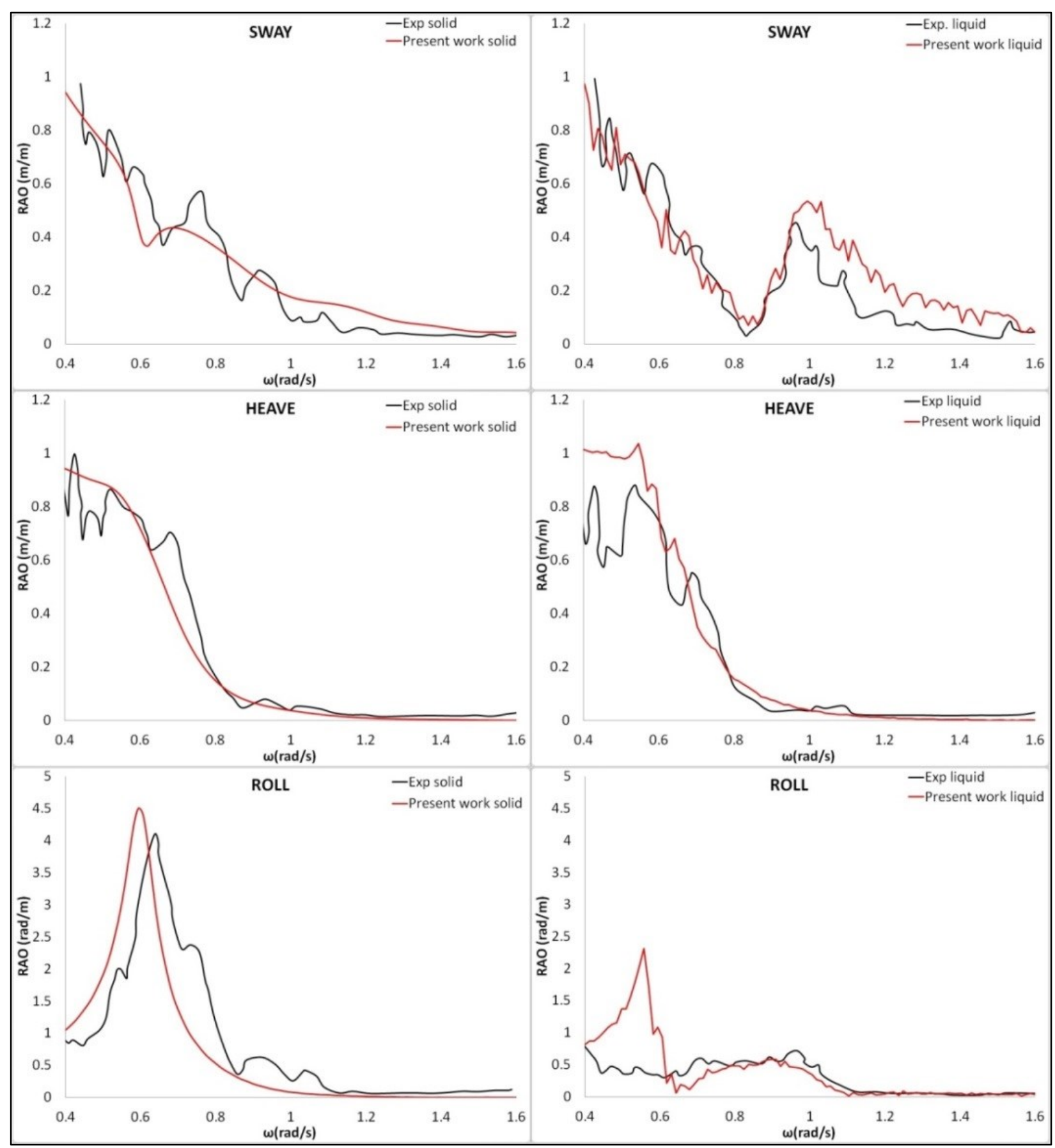

Figure 71: Experimental [110] versus present work. RAOs comparison. Solid case on the left, and liquid (coupled) case on the right. 


\section{Chapter 11. ANALYSIS OF FLOATING WIND TURBINES RESPONSE}

\subsection{Introduction}

Research trends in marine renewable energies have as one of its prominent topics the offshore wind energy. The technology for marine wind turbines is currently well-developed, but limited for fixed installations in shallow-water areas. The next horizon is focused on deep-water technology [111], but different challenges for Floating Offshore Wind Turbines (FOWT) are not solved yet [112], such as the dynamic stability in the presence of non-linear ambient loads [113]. In fact, an accurate prediction of the dynamic response of a FOWT, considering the interaction among the hydrodynamics, mooring, and aerodynamics of the turbine, is identified as one of the key challenges for the simulation tools required to design the future FOWTs $[114,115,116]$.

Standard design procedures and simulation tools for marine structures come from the existing technology and from experience of the oil and gas industry offshore. For instance, the classic simulation approaches are based on uncoupled formulations, where the hydrodynamic response of the floater is linearised and can be decoupled from the mooring [117,118]. Recently, coupled simulations have been adopted to solve the seakeeping of FOWT devices, since the dynamic of FOWT offers a high complexity due to the variety of loads and non-linear effects. Anyhow, the interaction among different components, such as the wind turbine structure, the rotor dynamics, the mooring arrangements, and the floating structure must be taken into account in a more accurate way [119].

The analysis of FOWTs should be carried out with simulation codes capable to include the physics governing the dynamic response of these devices. With regards to marine structures, Low and Langley [120] showed that the dynamic response of a floating production system in a random sea can be split in two timescales: low frequency and wave frequency responses. Moreover the seakeeping of the floating device and the dynamics of the mooring lines are coupled. Then, the analysis should take into account the interaction between them, and the time-domain analysis seems to be the choice way to simulate this sort of coupled problems. In fact, the American Bureau of Shipping [121] considers that the global seakeeping analysis of a FOWT should take into account: the unsteady wind loads, wind turbine control systems, wind turbine-platform interaction, wave actions over the platform, currents, mooring loads, and any other types of external actions. It will be presented later on that the timedomain approach allows handling these actions in a natural manner. 
The frequency-domain approach has difficulties to accurately handle non-linearities such as those arising from the mooring lines, and the low frequency components of the wave-body interaction, as appointed by Low [122], while the time-domain analysis can straightforwardly include any nonlinearity within each time step in a natural manner. Details on the mooring models used in this chapter were developed by Gutiérrez-Romero in [123].

\subsection{Validation of coupled seakeeping-mooring solver}

A fully coupled analysis of the OC3 spar buoy FOWT, named Hywind [124], is presented next. Main particulars of the FOWT are presented in $[125,126]$, and a general view of the buoy concept can be observed in Figure 72 .

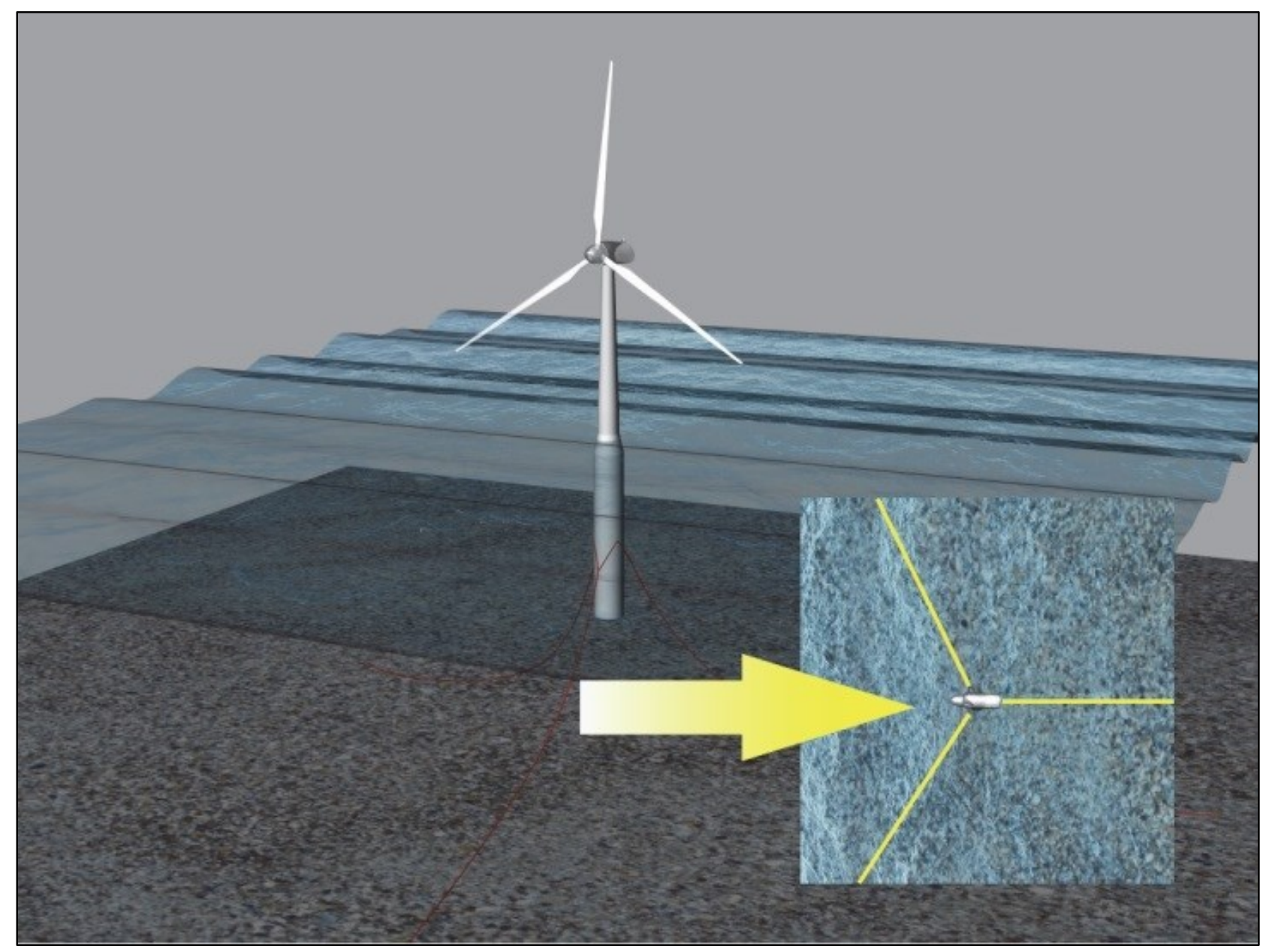

Figure 72: General view of the spar buoy wind turbine concept (OC3-Hywind concept).

\subsubsection{RAO Analysis}

First, a RAO analysis is performed in the absence of wind. The frequency results generated by the seakeeping solver are obtained after applying a Fourier transform to the resulting time history of 
displacements. Figure 73 shows an inter-code comparison, finding a good agreement among different solvers.

\subsubsection{Mooring analysis around pitch resonance}

Next the influence of three different mooring models on the OC3 spar buoy FOWT is analyzed. These models are: the linear cable model (that behaves as springs and is represented by a linearised mooring matrix [126]); the Quasi-Static model, similar to the one presented in [130]; and the dynamic model developed by Gutiérrez-Romero [123]. Six first-order monochromatic waves are used in the analysis. Key parameters of the mooring layout and cable properties can be found in Table 40 .

Figure 74 compares the pitch motion for each mooring model. It can be observed that there are big differences in the results around the pitch resonance $(\mathrm{Tw}=30 \mathrm{~s})$, while the results are quite similar for the cases with $\mathrm{Tw}=10 \mathrm{~s}$ and $\mathrm{Tw}=55 \mathrm{~s}$. These results suggest that the use of the linear model can lead to big errors near resonance, and to magnify safety factors that contribute to an increase of costs.

\subsubsection{Simulation in operational conditions}

Four analyses of the OC3 spar buoy in operational conditions are presented. The different analyses are carried out in similar environmental conditions, but using first-order and second-order irregular waves. Furthermore, additional studies including Quasi-Static and dynamic mooring models are performed. The goal of these analyses is to evaluate the effects of the mooring model and the wave order on the dynamics of the system, as well as to estimate tensions in the mooring lines.

On the one hand, the wind turbine system is assumed to be operating at an average wind speed of $11.4 \mathrm{~m} / \mathrm{s}$, which generates the maximum thrust and torque. FASTLognoter $[127,128]$ has been used to linearise with FAST [127], the behavior of the wind turbine around the operating wind speed. Restoring and damping matrices resulting from the linearization of the wind turbine system are included into the global dynamics. It should be remarked that the rotational and periodicity effects are considered in the calculation of the steady state matrix. In addition, the wind loads are estimated considering nonuniform wind flow, with an average wind speed of $11.4 \mathrm{~m} / \mathrm{s}$. The wind flow profile is obtained using Turbsim [129], and the wind loads on the wind turbine are obtained from FAST/AeroDyn [127]. A JONSWAP spectrum with a mean wave period $\mathrm{Tm}=12.0 \mathrm{~s}$, and significant wave height $\mathrm{Hs}=6.0 \mathrm{~m}$ is considered. The key parameters of the different case studies are presented in Table 41.

Two different types of mooring models are analyzed; one based on the Quasi-Static catenary model [130], and the other based on the dynamic cable model solved by a non-linear FEM formulation (NFEM) [123]. For the dynamic cable analysis, each mooring line is divided into 200 elements. Figure 75, Figure 76, and Figure 77 show the computed heave, roll and pitch motions for $300 \mathrm{~s}$. Noticeable 
differences are found between the first-order and second-order movements, while the quasistatic (QS) and NFEM mooring models offer quite similar results. Table 42 shows the mean and RMS values, as well as the motion amplitude for the first-order and second-order movements. When comparing the QS and the NFEM models, only slight differences are observed. In particular, the second-order pitch motion is higher when using the NFEM model, compared to the QS model, while the other values remain with similar trends for both models. Figure 78 shows the tension for each mooring line at the fairlead point. Table 43 compares the maximum, minimum, average, and RMS tension values at the fairlead points, obtaining similar values for both mooring models. It is emphasized that the NFEM mooring model allows considering dynamic effects which are not taken into account by QS models. However, in deep water, it was reported [120] that dynamic effects are negligible for catenary type lines.

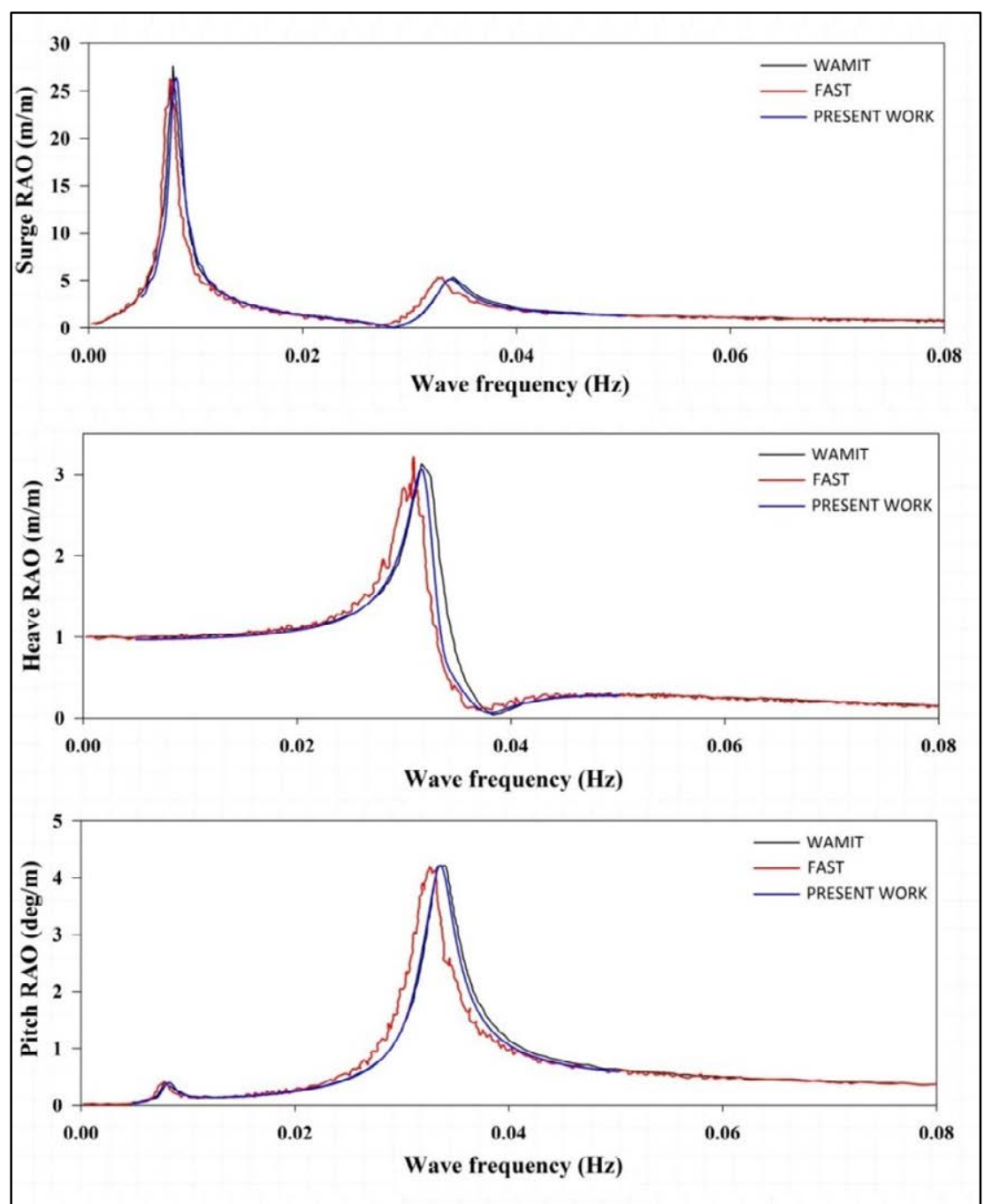

Figure 73: OC3-Hywind concept. Comparison between the computed results with those from [131] for a rigid wind turbine with no wind. 
Table 40: Key parameters of pitch resonance study [126]

\begin{tabular}{|c|c|c|}
\hline & Item Value & Value \\
\hline \multicolumn{2}{|c|}{ Wave amplitude [m] } & $1.0 \mathrm{~m}$ \\
\hline \multicolumn{2}{|c|}{ Wave period $[\mathrm{s}]$} & $10,20,25,35,40,55$ \\
\hline \multicolumn{2}{|c|}{ Number of mooring lines } & 3 \\
\hline \multicolumn{2}{|c|}{ Angle between adjacent lines $\left[{ }^{\circ}\right]$} & 120 \\
\hline \multicolumn{2}{|c|}{ Anchors depth (below SWL) [m] } & 320 \\
\hline \multicolumn{2}{|c|}{ Fairleads depth (below SWL) [m] } & 70 \\
\hline \multicolumn{2}{|c|}{ Fairleads radius from Centerline [m] } & 853.9 \\
\hline \multirow{4}{*}{ Mooring line } & Unstretched length [m] & 902.2 \\
\hline & Diameter [m] & 0.9 \\
\hline & Linear mass density $[\mathrm{kg} / \mathrm{m}]$ & 77.71 \\
\hline & Extensional stiffness [N] & $3.84 \times 10^{8}$ \\
\hline
\end{tabular}

\subsection{Summary}

The Hywind FOWT has been analyzed under realistic operational conditions. Second-order coupled simulations have been carried out considering a real wind profile, and with two types of mooring models; the QS and the NFEM. Results from both models have been compared, and the comparison suggests that using a QS model for the fatigue assessment of mooring lines could overestimate their fatigue life.

Table 41: Key parameters of fully coupled simulations for Hywind floating offshore wind turbine

\begin{tabular}{|c|c|c|c|c|}
\hline & Case 1 & Case 2 & Case 3 & Case 4 \\
\hline Average wind velocity $[\mathrm{m} / \mathrm{s}]$ & 11.4 & 11.4 & 11.4 & 11.4 \\
\hline Wind direction $\left[{ }^{\circ}\right]$ & 0 & 0 & 0 & 0 \\
\hline Wave spectrum & Jonswap 1st & Jonswap 1st & Jonswap 2nd & Jonswap 2nd \\
\hline Significant wave height [m] & 6.0 & 6.0 & 6.0 & 6.0 \\
\hline Peak period [s] & 12 & 12 & 12 & 12 \\
\hline Mean wave direction $\left[{ }^{\circ}\right]$ & 0 & 0 & 0 & 0 \\
\hline Mooring model & QS & Nonlinear-FEM & QS & Nonlinear-FEM \\
\hline Number of mooring lines & 3 & 3 & 3 & 3 \\
\hline Number of elements per line & 200 & 200 & 200 & 200 \\
\hline
\end{tabular}




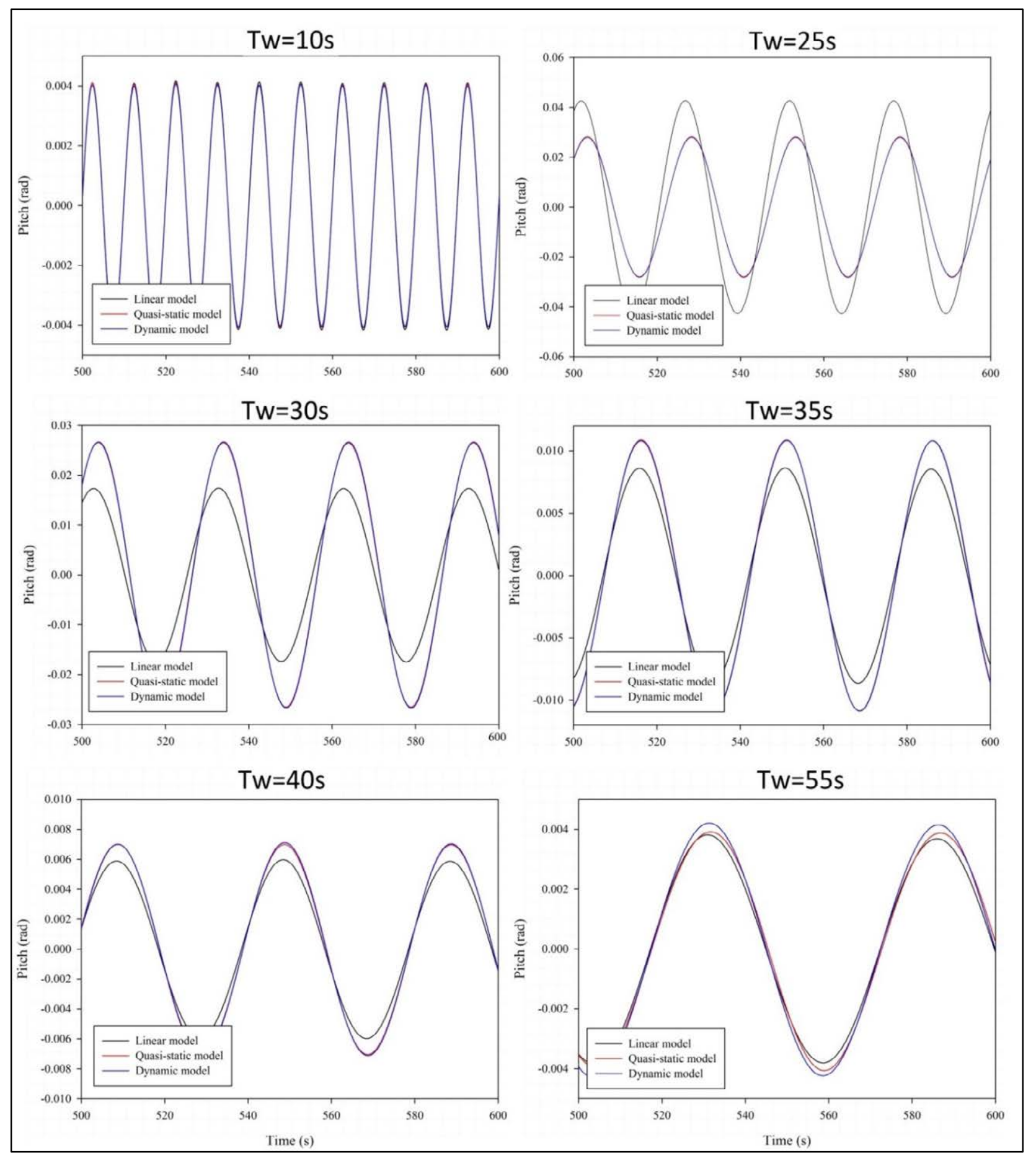

Figure 74: Results obtained for mooring analysis around pitch resonance of OC3-Hywind. 


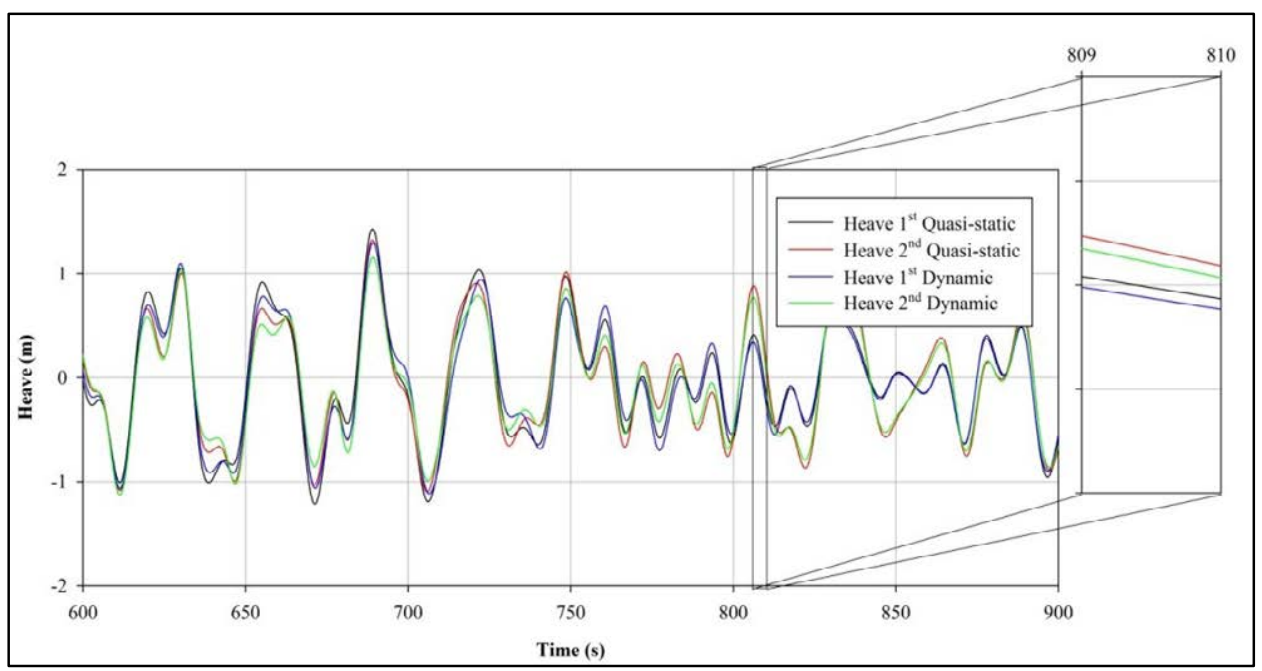

Figure 75: Comparison between heave motion for first and second-order wave environment for Case 1-4

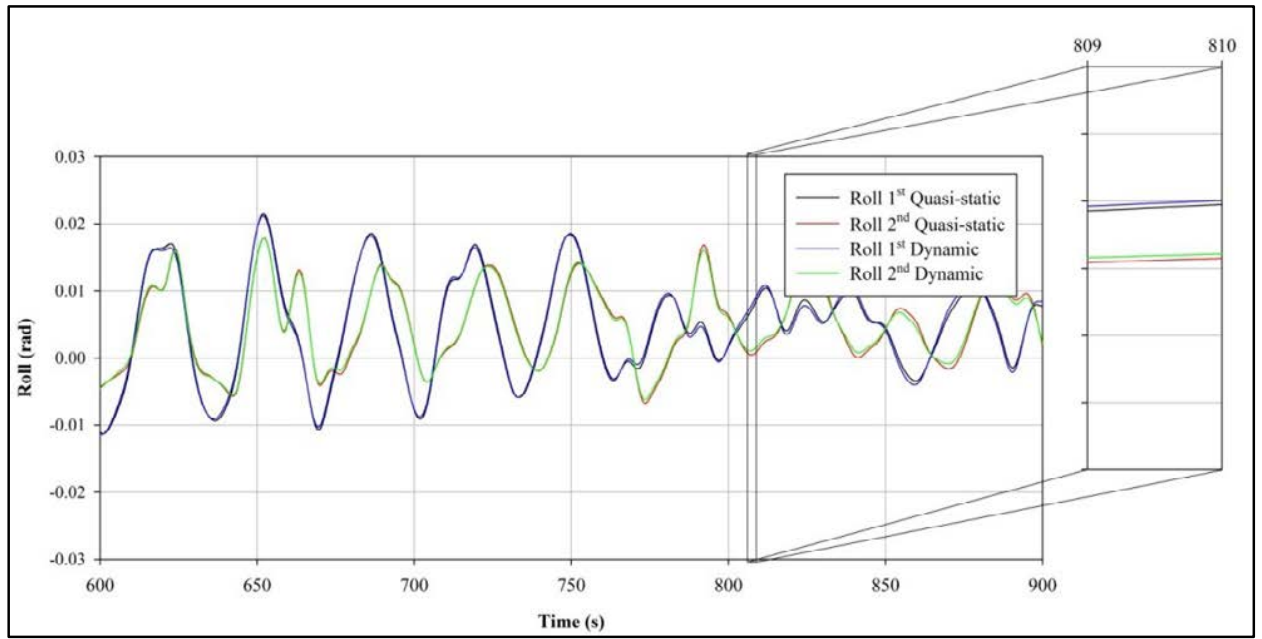

Figure 76: Comparison between roll motion for first and second-order wave environment for Case 1-4.

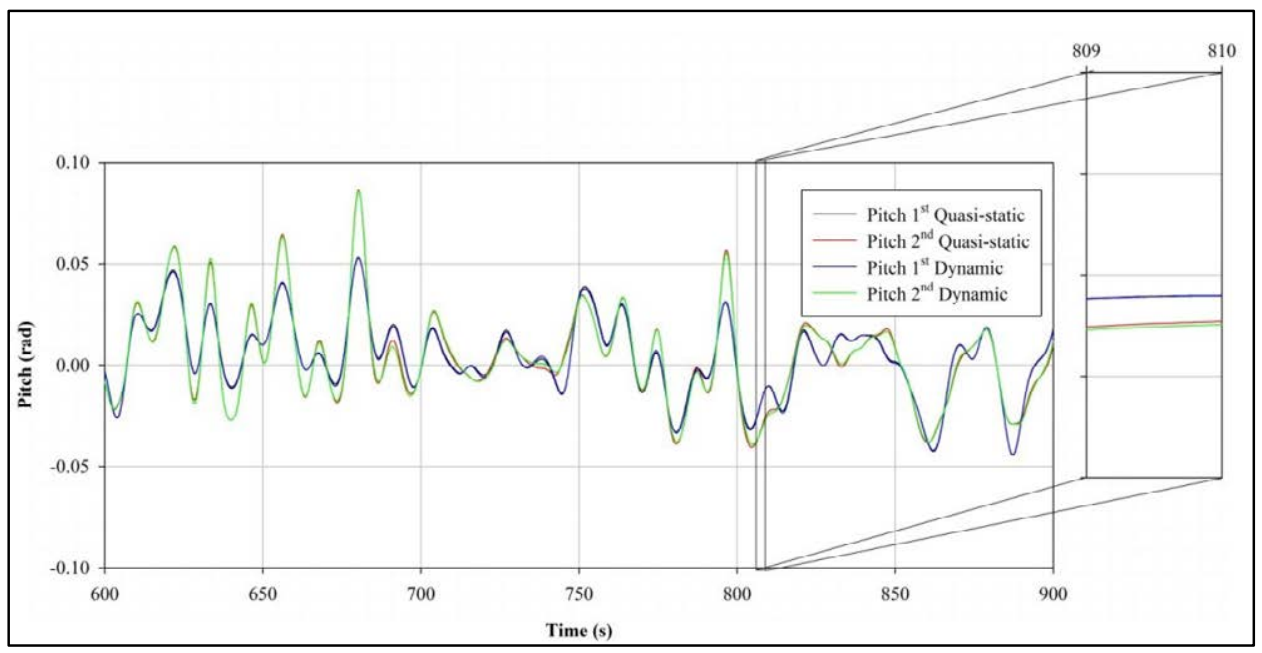

Figure 77: Comparison between pitch motion for first and second-order wave environment for Case 1-4. 


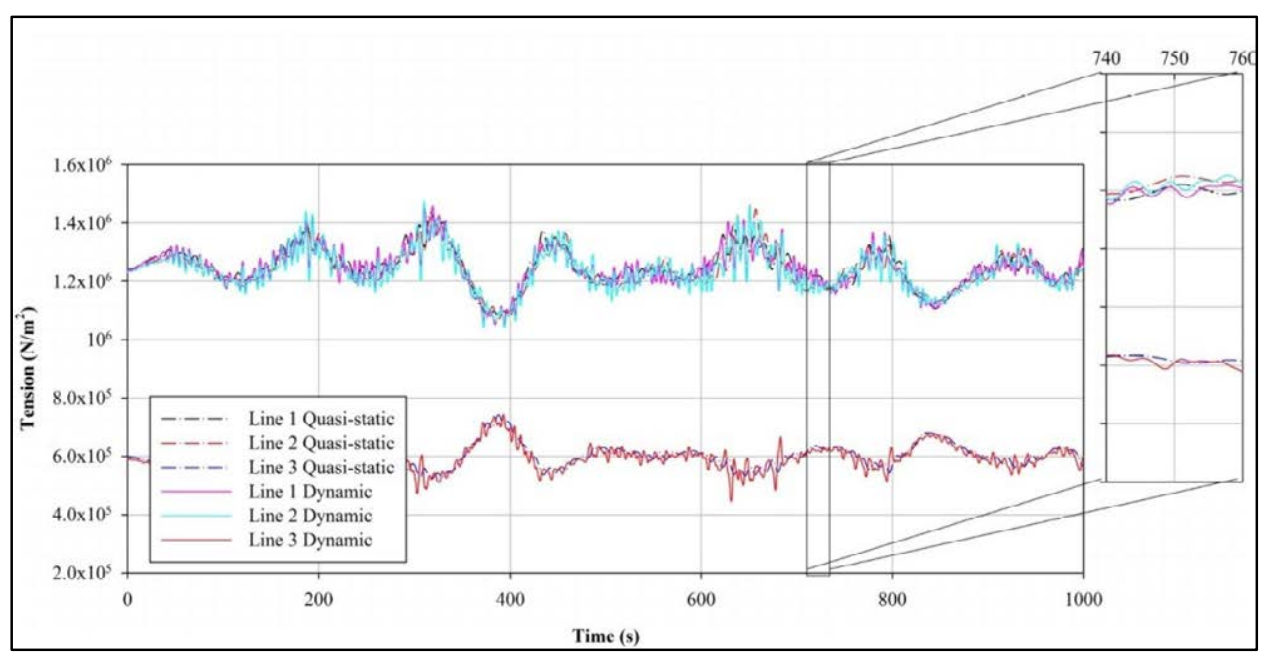

Figure 78: Comparison of fairlead tension of each mooring line for Cases 3 and 4.

Table 42: Comparison between mean, amplitude and RMS values of FOWT motions for first and second-order wave environment

\begin{tabular}{|c|c|c|c|c|c|c|}
\hline \multicolumn{7}{|c|}{ QS } \\
\hline & Surge [m] & Sway [m] & Heave [m] & Roll [deg] & Pitch [deg] & Yaw [deg] \\
\hline Mean 1st & 0.04 & 0.00 & 0.00 & 0.29 & 0.41 & 0.21 \\
\hline Mean 2nd & 0.04 & 0.00 & 0.00 & 0.29 & 0.41 & 0.21 \\
\hline Amplitude 1st & 14.96 & 1.34 & 2.75 & 1.91 & 6.12 & 10.38 \\
\hline Amplitude 2nd & 14.96 & 1.34 & 2.75 & 1.91 & 6.12 & 10.38 \\
\hline RMS 1st & 2.76 & 0.21 & 0.53 & 0.52 & 1.09 & 1.92 \\
\hline RMS 2nd & 2.73 & 0.24 & 0.45 & 0.48 & 1.22 & 1.91 \\
\hline \multicolumn{7}{|c|}{ NFEM } \\
\hline & Surge [m] & Sway [m] & Heave [m] & Roll [deg] & Pitch [deg] & Yaw [deg] \\
\hline Mean 1st & 0.00 & 0.00 & -0.01 & 0.29 & 0.40 & 0.21 \\
\hline Mean 2nd & 0.10 & 0.00 & -0.03 & 0.30 & 0.42 & 0.28 \\
\hline Amplitude 1st & 13.82 & 1.12 & 2.61 & 1.92 & 6.03 & 10.31 \\
\hline Amplitude 2nd & 15.19 & 1.55 & 2.59 & 2.02 & 8.00 & 10.83 \\
\hline RMS 1st & 2.54 & 0.18 & 0.50 & 0.5 & 1.09 & 1.92 \\
\hline RMS 2nd & 2.73 & 0.24 & 0.45 & 0.48 & 1.22 & 1.91 \\
\hline
\end{tabular}

Table 43: Comparison between maximum, minimum, max-min, mean, and RMS values of fairlead tension for Quasistatic and Dynamic mooring models

\begin{tabular}{|c|c|c|c|c|c|c|}
\hline Line & Case & Max [MN] & Min [MN] & Max-Min[MN] & Mean [MN] & RMS [MN] \\
\hline $\mathbf{1}$ & 3 & 1.435 & 1.073 & 0.362 & 1.250 & 1.252 \\
\hline $\mathbf{2}$ & 3 & 1.446 & 1.072 & 0.374 & 1.240 & 1.242 \\
\hline $\mathbf{3}$ & 3 & 0.7411 & 0.5209 & 0.220 & 0.5991 & 0.6003 \\
\hline $\mathbf{1}$ & 4 & 1.457 & 1.052 & 0.405 & 0.1246 & 1.248 \\
\hline $\mathbf{2}$ & 4 & 1.471 & 1.040 & 0.431 & 1.236 & 1.238 \\
\hline $\mathbf{3}$ & 4 & 0.7421 & 0.4510 & 0.291 & 0.5923 & 0.5936 \\
\hline
\end{tabular}




\section{Chapter 12. ANALYSIS OF AN OSCILLATING WATER COLUMN DEVICE}

\subsection{Introduction}

Nowadays, harvesting marine energy is being investigated in order to obtain clean and renewable energy. Among all the designs for that purpose, one of the most relevant is the one based on the oscillating water column (OWC) principle. This chapter aims at showing how to analyze the energy extraction performance of an oscillating water column device with the simulation tools developed in this thesis.

\subsection{Oscillating water column device}

The OWC device considered in this thesis consists of an empty circular column, completely opened at its base, and with a small opening at the top side that works as an inlet for a turbine. The column is placed fixed in the sea under the action of waves, which induce an oscillating movement of the free surface trapped inside the column. This oscillating movement induces airflow across the turbine, which converts the wave energy into mechanical energy. Moreover the pressure inside the OWC changes and this pressure variation is to be taken into account within the dynamic free surface condition. Figure 79 shows the geometry and main particulars of the device to be analyzed.

\subsection{Environment and computational domain}

A $10 \mathrm{~m}$ deep water domain is considered. In order to be able to solve accurately the wave diffraction problem, a mesh size of $0.2 \mathrm{~m}$ for the OWC device and a mesh size of $0.5 \mathrm{~m}$ for the near field volume are used. A wave damping zone is created starting at 7 meters away from the OWC centerline in order to dissipate the diffracted waves. The resulting mesh consists of 52388 nodes and 301545 elements (see Figure 80).

\subsection{Turbine model}

A wells type turbine has been chosen for the analysis of the OWC turbine. The pressure drop across the turbine and the power generated are modeled with the following equations: 


$$
\begin{aligned}
& p=\frac{Q|Q|}{0.006096}, \\
& P=-0.0051\left|\frac{p}{1000}\right|^{3}+0.2815\left(\frac{p}{1000}\right)^{2}+1.1857\left|\frac{p}{1000}\right|,
\end{aligned}
$$

where $Q$ is the airflow across the turbine in $\mathrm{m}^{3} / \mathrm{s}, p$ is the pressure inside the OWC chamber in $\mathrm{Pa}$, and $\mathrm{P}$ is the power generated by the turbine in Kw. Figure 81 shows the characteristics curves of the turbine model.

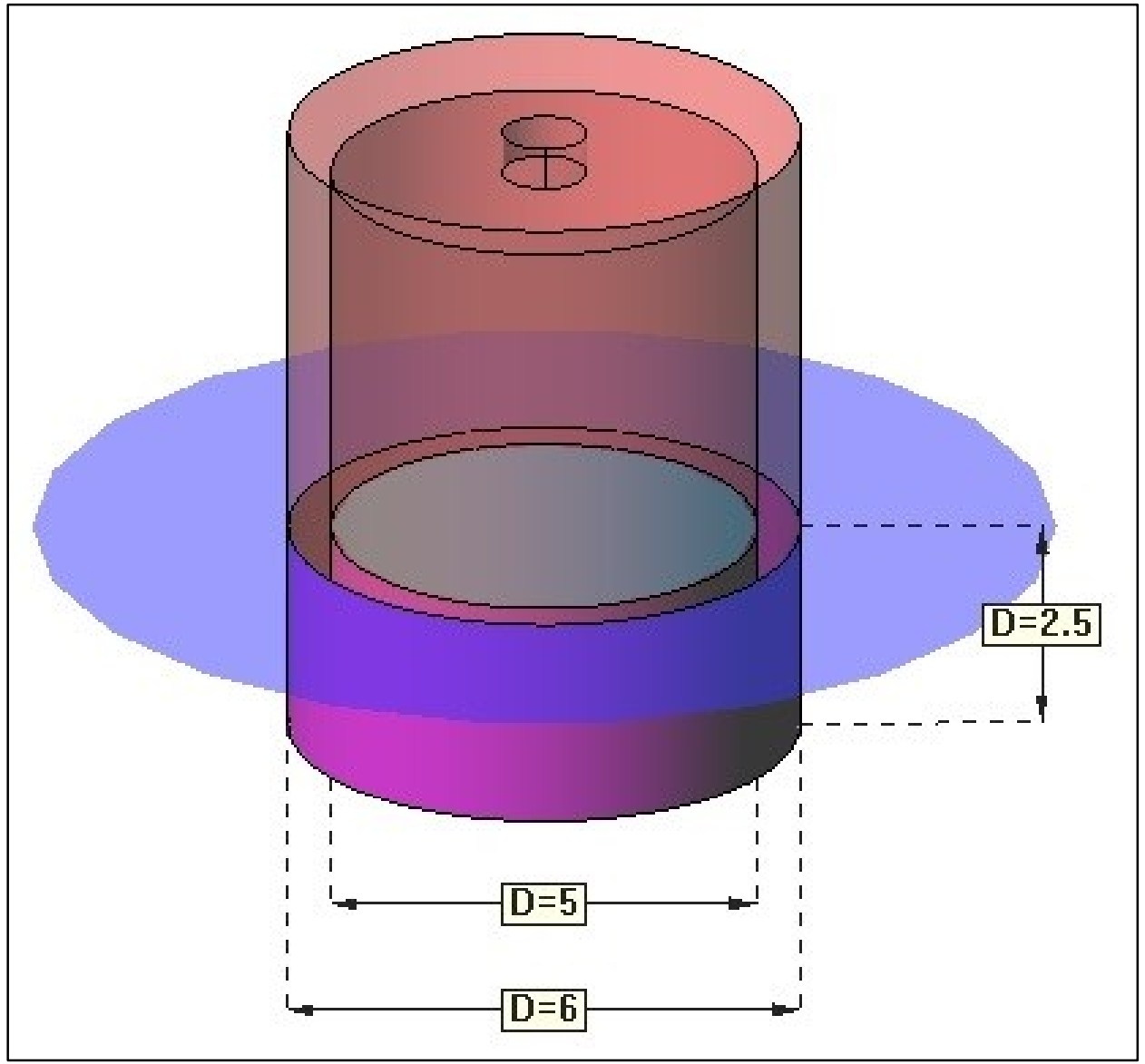

Figure 79: OWC particulars (lengths in meters).

The airflow is instantaneously calculated by derivation respect to time of the free surface volume variation within the OWC chamber. Then, the corresponding instantaneous pressure is imposed in the wave diffraction problem via the dynamic free surface boundary condition.

\subsection{RAO analysis}

In a first step of the analysis, the OWC column if subject to a white noise wave spectrum in the absence of the turbine in order to analyze water level response inside the OWC at different wave periods. Figure 82 shows the RAOs obtained for different wave periods. It can be observed that there 
is a resonance peak at $T=3.95 \mathrm{~s}$ approximately, while the RAO goes to zero for short waves and to unity for long waves.

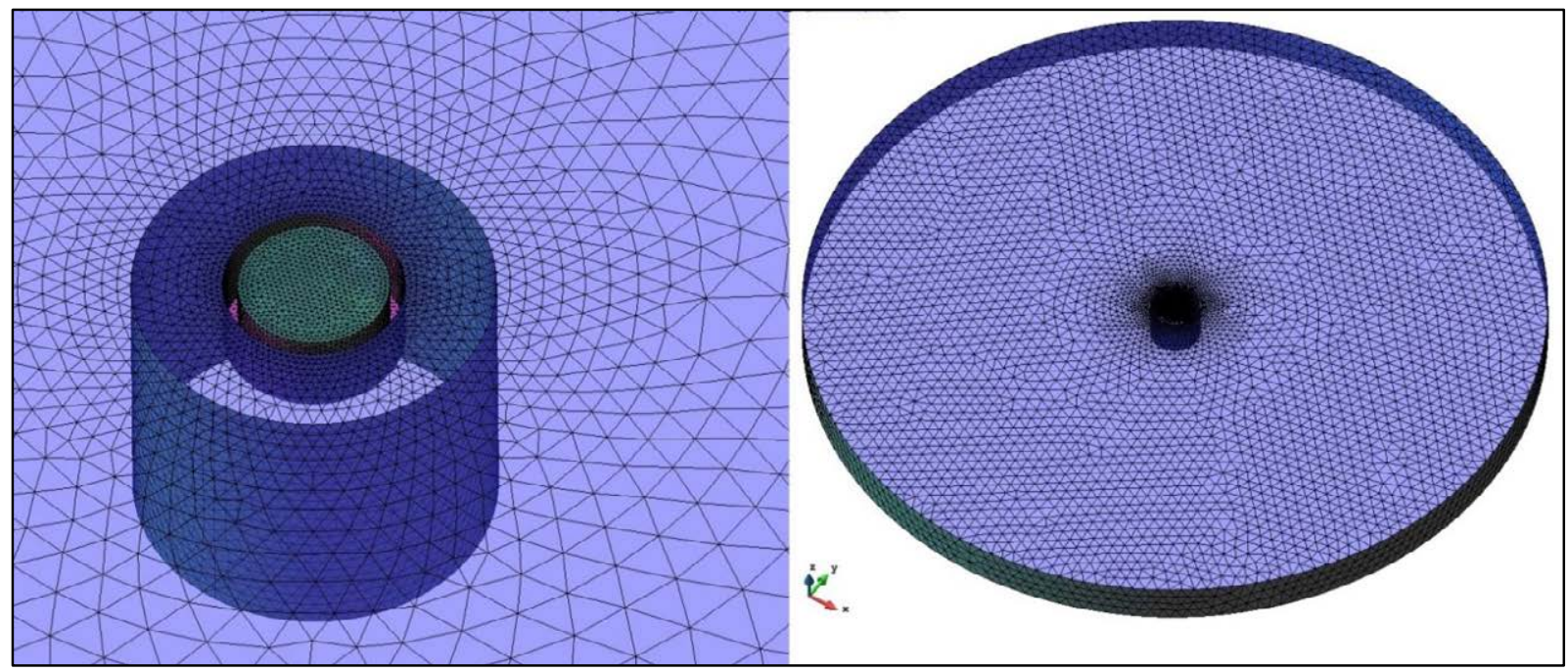

Figure 80: Left: OWC mesh close up. Right: computational domain mesh.
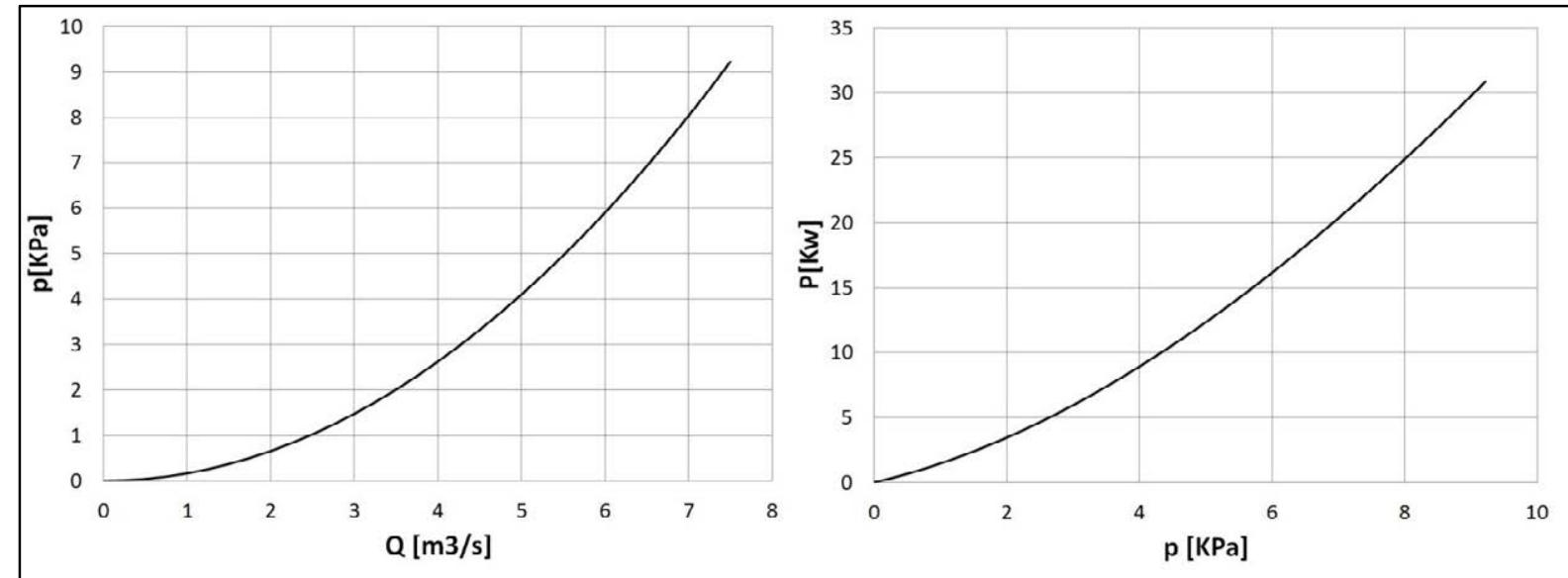

Figure 81: PTO model.

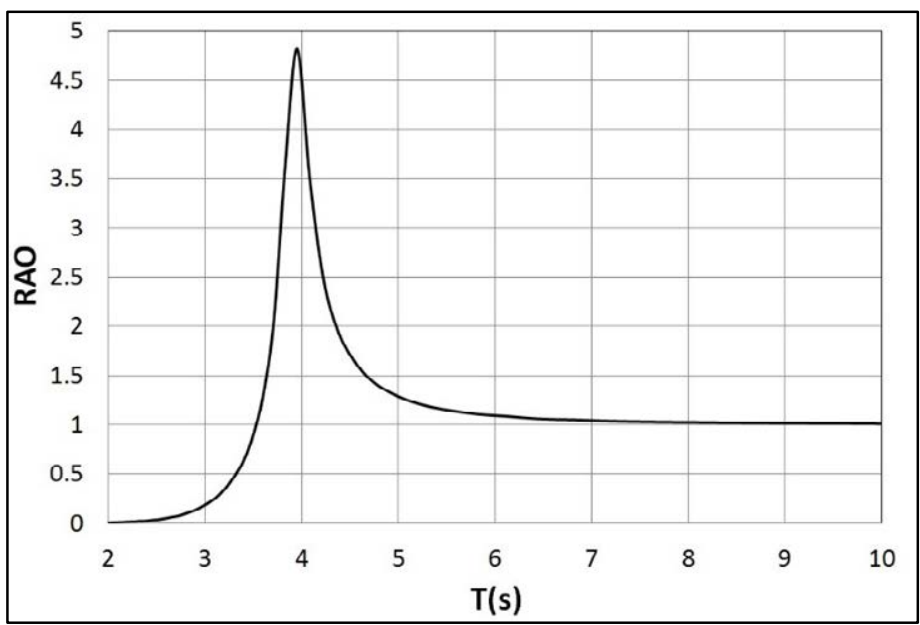

Figure 82: RAOs for OWC device. 


\subsection{Analysis in irregular waves}

Table 44 shows the main particular of the environmental conditions under which the OWC is to be analyzed. The OWC resonant period has been chosen as the mean wave period. The analysis time has been set to 300s. Figure 83 represents a snapshot of the free surface elevation outside and inside the WC device. Figure 84 shows the instantaneous flow Q, pressure inside the chamber p, power generated $\mathrm{P}$, and the average power $P_{\text {ave }}$ produced till the corresponding time. For the given sea state, turbine, and $\mathrm{OWC}$ geometry, the average power produced is in the order of $1 \mathrm{Kw}$.

Table 44: Environmental particulars

\begin{tabular}{|c|c|}
\hline Water depth & $10 \mathrm{~m}$ \\
\hline Spectrum type & Jonswap \\
\hline Mean wave period & $3.95 \mathrm{~s}$ \\
\hline Significant wave height & $1 \mathrm{~m}$ \\
\hline Wave spreading angle & $60 \mathrm{deg}$ \\
\hline
\end{tabular}

\subsection{Summary}

A specific OWC device with a circular cylinder shape has been analyzed. First, a RAO analysis was carried out in order to find where resonance effect occurs. Second, an estimation of the power production under irregular sea conditions has been performed. Notice that the turbine equations used here are non-linear, leading to a non-linear response when solving the wave diffraction problem. For this reason, a time domain approximation as the one used here is an appropriate approach to handle this sort of non-linearities.

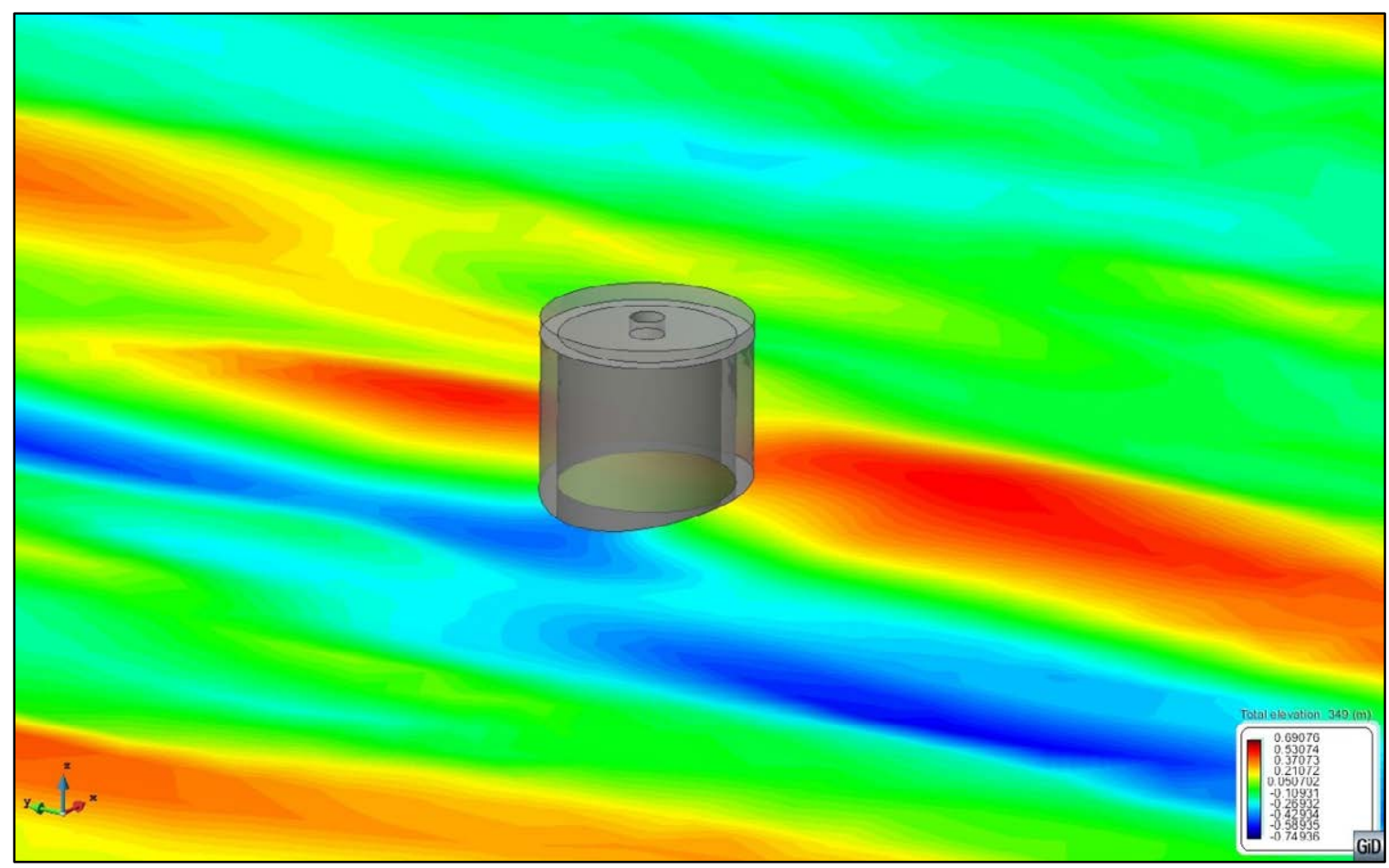

Figure 83: Irregular sea and OWC device. 

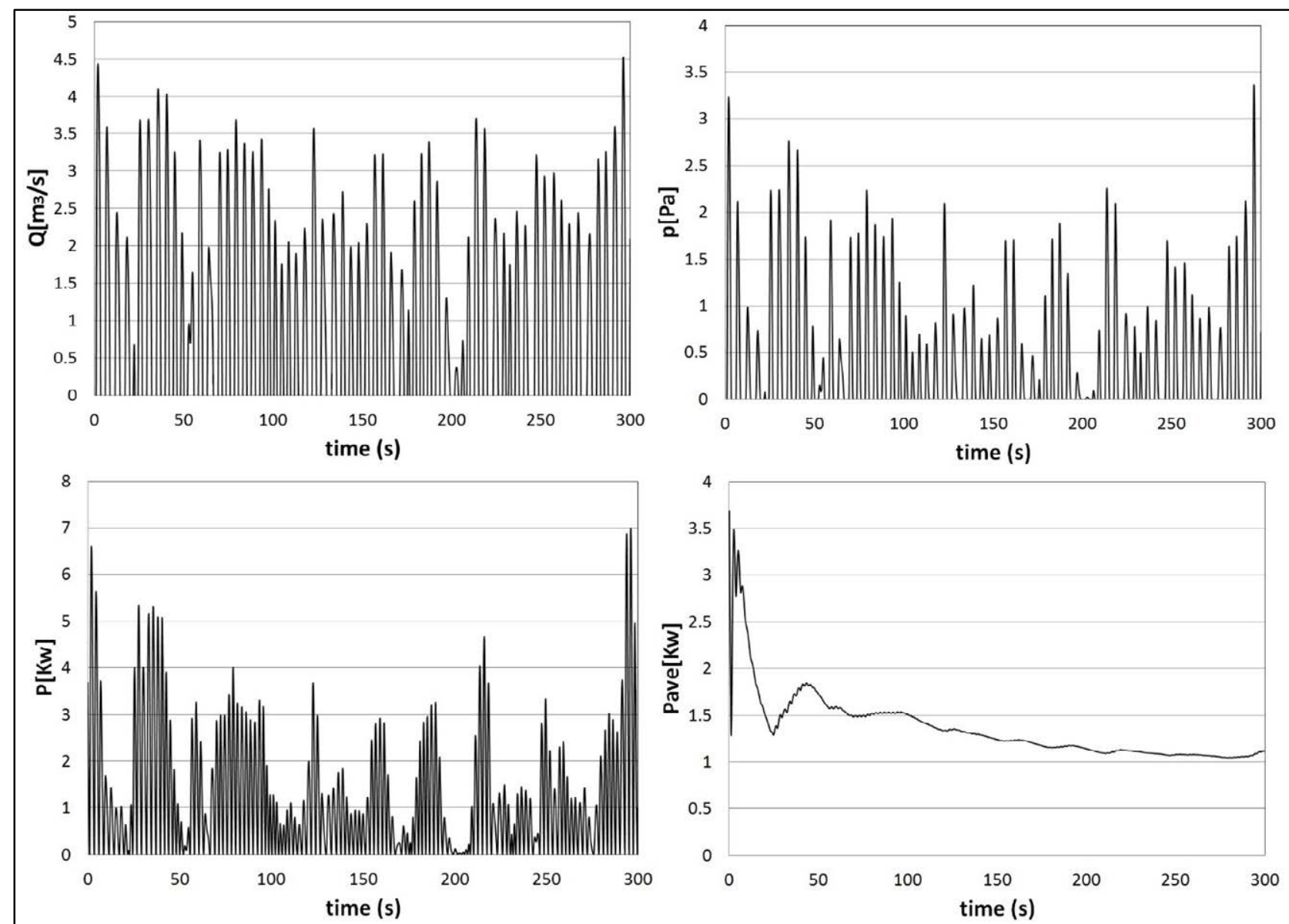

Figure 84: OWC outputs; instantaneous airflow (upper-left); instantaneous pressure (upper-right); instantaneous power (lower-left); and average power (lower-right). 


\section{Chapter 13. ANALYSIS OF A PELAMIS TYPE DEVICE}

\subsection{Introduction}

Among all the designs conceived for extracting energy from waves, the Pelamis, manufactured by Pelamis Wave Power, is a snake shape artefact that converts wave energy through the relative movements between bodies of a multibody system (see Figure 85). Nowadays, several full scale Pelamis devices have been deployed and are being tested.

The Pelamis type consists of a set of bodies connected by joints and with a snake shape. This system will rearrange its shape due to the action of waves, and therefore energy can be extracted from the relative movement across consecutive segments. This chapter aims at showing how to analyze the energy extraction performance of Pelamis type device.

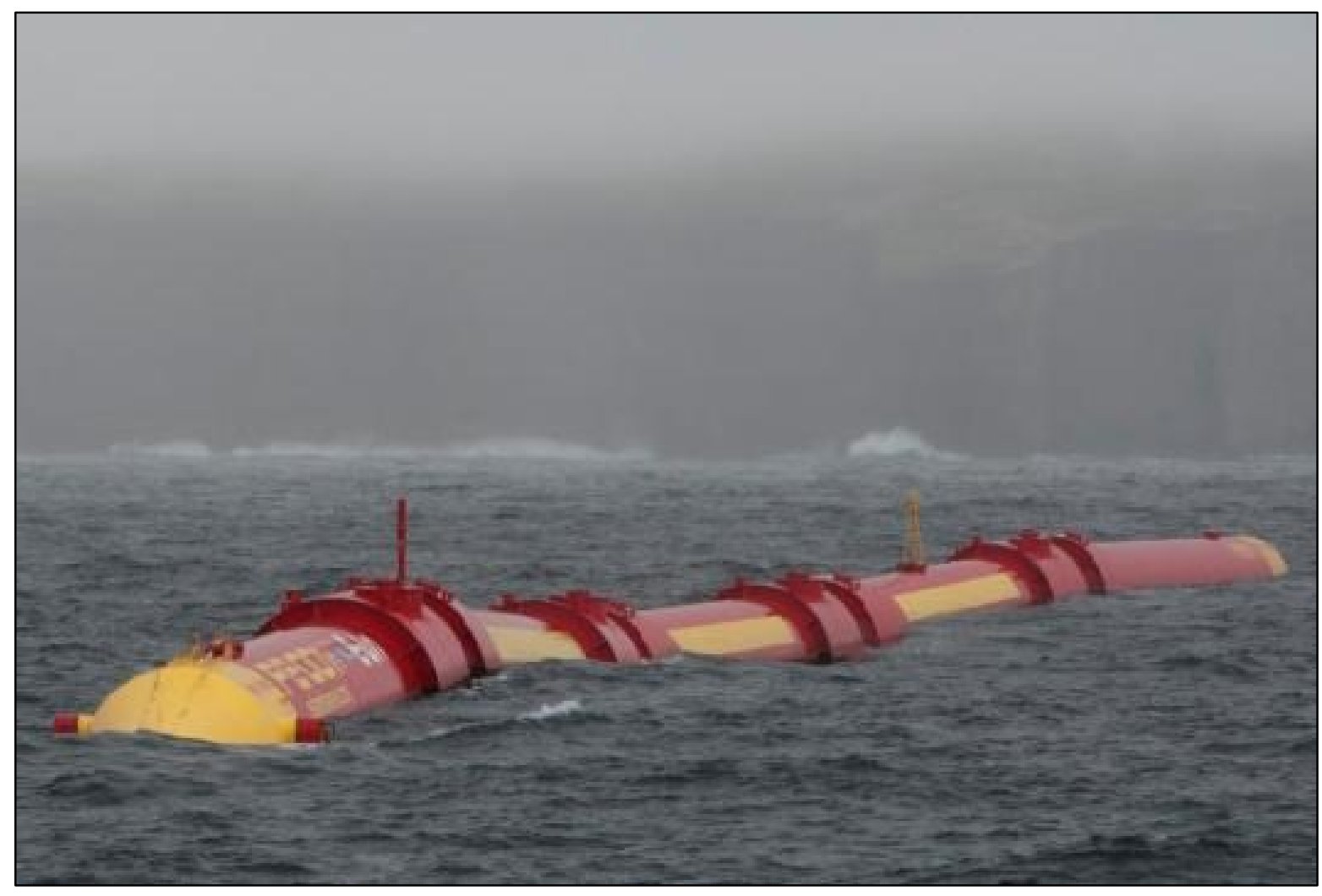

Figure 85: Pelamis device. 


\subsection{Pelamis type device}

The device considered in this thesis is a very simplify model of the actual Pelamis. It consists of two circular cylinders connected by a hinge type connector. Then, an energy extracting system is used to extract energy from the relative pitch velocity, acting as well as a pitch damper. Its main particulars are shown in Table 45 while Figure 86 shows the geometry of the connector and power take off (PTO) system.

Table 45: Segments particulars

\begin{tabular}{|c|c|}
\hline Length & $34 \mathrm{~m}$ \\
\hline Beam & $5.5 \mathrm{~m}$ \\
\hline Draft & $3.404 \mathrm{~m}$ \\
\hline Distance between segments & $1.5 \mathrm{~m}$ \\
\hline XG (from stern side) & $17 \mathrm{~m}$ \\
\hline YG & 0 \\
\hline ZG & -1 \\
\hline Radii of inertia $\mathbf{r}_{\mathbf{x x}}$ & $2.4 \mathrm{~m}$ \\
\hline Radii of inertia $\mathbf{r}_{\mathbf{y y}}=\mathbf{r}_{\mathbf{z z}}$ & $12.3 \mathrm{~m}$ \\
\hline
\end{tabular}

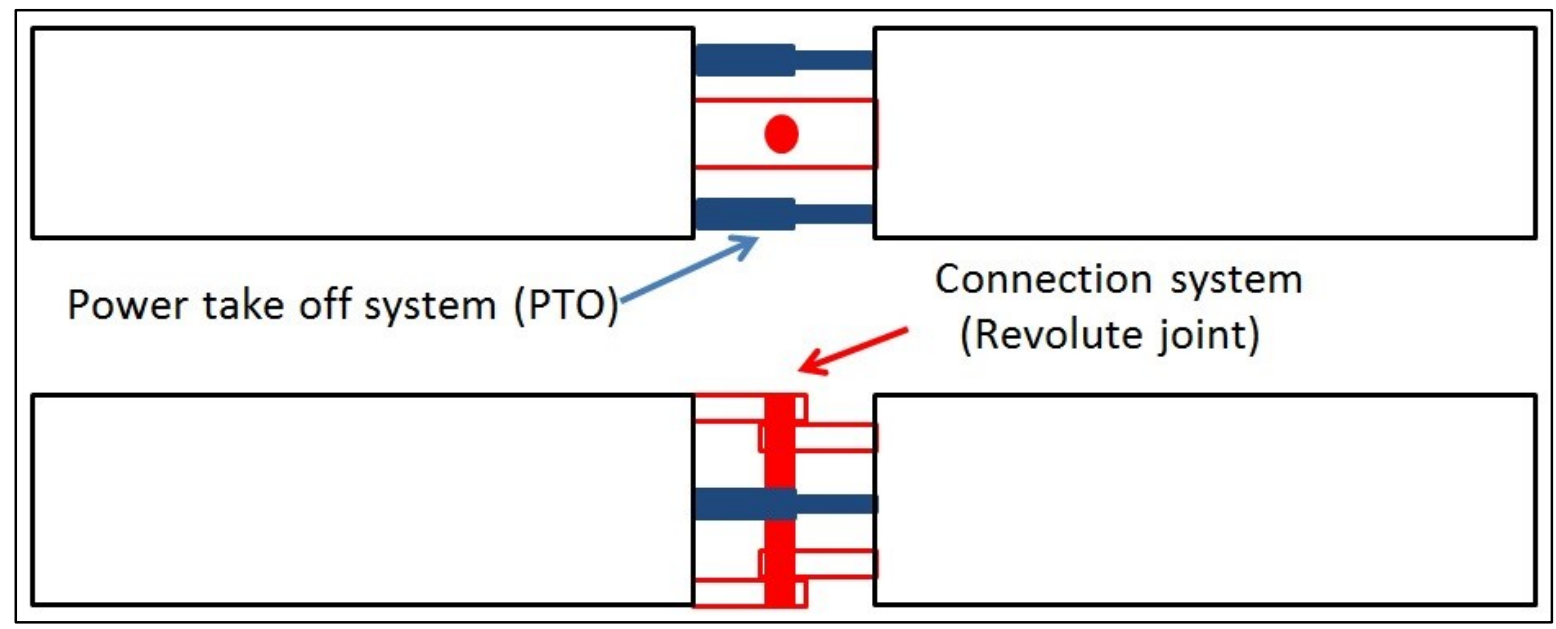

Figure 86: Connection and PTO. Top: front view; down: top view

\subsection{Connection system}

\subsubsection{Spherical joint}

Two elements are said to be linked by a spherical joint when they have one point each $\left(P_{1}\right.$ and $\left.P_{2}\right)$ occupying the same position in space regardless of the body movements. This condition can be written in vector form as (see Figure 87):

$$
\overrightarrow{O P_{1}}=\overrightarrow{O G_{1}}+\overline{\bar{R}}^{(1)} \overrightarrow{G_{1} P}=\overrightarrow{O G_{2}}+\overline{\bar{R}}^{(2)} \overrightarrow{G_{2} P}=\overrightarrow{O P_{2}},
$$

where $\overline{\bar{R}}^{(i)}$ is the rotation matrix of the $i$ element. Eq. (13-1) consists of three equations, one for each coordinate. 
The rotation matrix is defined using the Tait-Bryan angles following the roll-pitch-yaw criterion: $\overline{\bar{R}}=\overline{\bar{R}}_{z} \overline{\bar{R}}_{y} \overline{\bar{R}}_{x}$

where $\overline{\bar{R}}_{x}, \overline{\bar{R}}_{y}$, and $\overline{\bar{R}}_{z}$ are the rotation matrices for pure rotations around the $\mathrm{x}, \mathrm{y}$, and $\mathrm{z}$ axis.

Equation (13-1) can be written as a set of three equations, one for each coordinate:

$$
\begin{aligned}
f_{i}\left(\boldsymbol{x}^{(1)}(t), \boldsymbol{x}^{(2)}(t)\right)= & x_{i}^{(1)}+r_{i 1}^{(1)} p_{x}^{(1)}+r_{i 2}^{(1)} p_{y}^{(1)}+r_{i 3}^{(1)} p_{z}^{(1)} \\
& -x_{i}^{(2)}-r_{i 1}^{(2)} p_{x}^{(2)}-r_{i 2}^{(2)} p_{y}^{(2)}-r_{i 3}^{(2)} p_{z}^{(2)}=0,
\end{aligned}
$$

where $x_{i}^{(j)}$ represent the linear displacements of element $\mathrm{j}, r_{i 1}^{(j)}$ is the matrix coefficient of the rotation matrix for element $\mathrm{j}$, and $p_{x}^{(j)}, p_{y}^{(j)}, p_{z}^{(j)}$ are the initial coordinate of point $\mathrm{P}$ respect to element $\mathrm{j}$. Coefficients $r_{i 1}^{(j)}$ depend on the roll, pitch, and yaw rotation angles.

\subsubsection{Revolute joint}

In the specific case of this work, the connecting system works as a revolute joint (or hinge). This joint restricts five degrees of freedom, therefore a five constraint equations system must be defined. The revolute joint is modeled as two spherical joints located on the rotation axis. However, two spherical joints require six equations instead of five, and one must be discarded since otherwise the system of equations would not be linearly independent.

In this specific case, the initial rotation axis is parallel to the y axis. Then, the equations regarding the displacement of the joints in the y direction are redundant, and one of them must be eliminated. As a result, five constraints equations are left defining the hinge between segments. Once the constraints equations of the revolute joint have been obtained, they are introduced within the body dynamics of the system via Lagrange multipliers as described in section 4.5.

\subsection{PTO model}

Real PTO models of actual Pelamis devices are quite complex and information regarding how they work is not publicly available. Hence in this demonstration case and for the sake of simplicity, a simpler PTO model will be used.

The PTO is assumed to work as a pitch damper, where the reaction of the dampers produce a pitch moment in the opposite direction of the relative pitch velocity, and whose magnitude is proportional to the relative velocity. The mathematical model describing this system is:

$$
\begin{aligned}
& M_{y}^{(1)}=-K_{\text {PTO }}\left(\omega_{y}^{(1)}-\omega_{y}^{(2)}\right), \\
& M_{y}^{(2)}=-K_{\text {PTO }}\left(\omega_{y}^{(2)}-\omega_{y}^{(1)}\right),
\end{aligned}
$$


$P=K_{P T O}\left(\omega_{y}^{(2)}-\omega_{y}^{(1)}\right)^{2}$,

where $M_{y}^{(i)}$ and $\omega_{y}^{(i)}$ represent the pitch movements and angular velocity for segment $(i), K_{\text {PTO }}$ is a constant value depending on the PTO design, and P is the instantaneous power extracted.

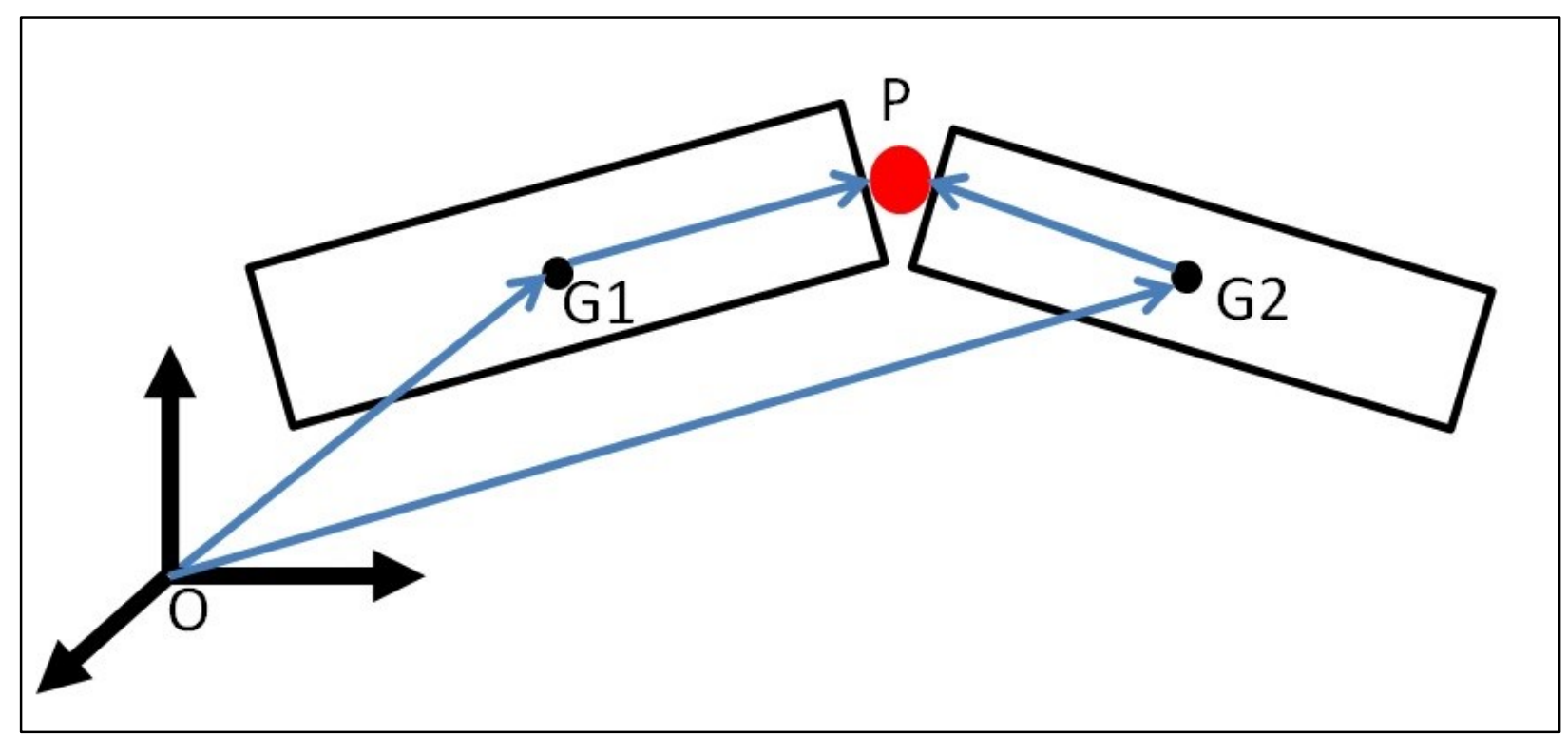

Figure 87: Spherical joint.

The PTO system is taken into account within the body dynamics by introducing the corresponding pitch moment as an external moment. Hence the PTO pitch moment is updated at every iteration of the body dynamics solver.

\subsection{Computational domain}

A $100 \mathrm{~m}$ deep fluid domain is considered. A mesh size of $0.5 \mathrm{~m}$ for the device and surroundings and a mesh size of $5 \mathrm{~m}$ for the near field volume have been used. A damping zone is located starting at 200 meters away from the device. The resulting mesh consists of 135153 nodes and 784579 elements (see Figure 88).

\subsection{Wave environment and mooring system}

\subsubsection{Wave environment}

The wave environment is considered an irregular sea modelled with a Jonswap spectrum with a mean wave period of $11 \mathrm{~s}$, significant wave height of $2.5 \mathrm{~m}$, and a wave spreading angle of 30 degrees. The mean direction of propagation is along the $\mathrm{x}$ axis, which is parallel to the longitudinal direction of the device. A total number of 55 monochromatic waves have been used to reproduce the irregular sea. 


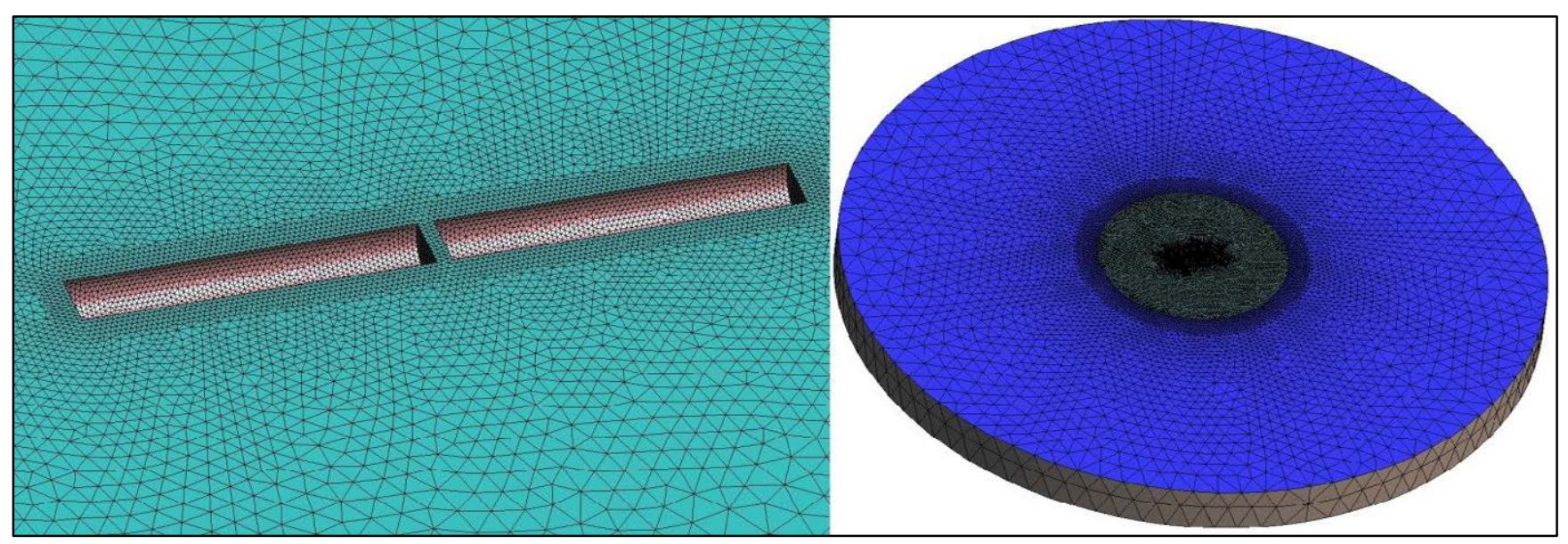

Figure 88: Left: Device mesh close up. Right: computational domain mesh.

\subsubsection{Mooring system}

The device is moored by means of three catenary lines. All of them are connected to the bow of the first segment, and then spread towards the sea bottom with a spreading angle of $120^{\circ}$ between lines. The main particulars of the mooring system are given in Table 46.

Table 46: Mooring lines particulars

\begin{tabular}{|c|c|}
\hline Length & $267.4 \mathrm{~m}$ \\
\hline Sectional area & $7.85398 \mathrm{E}-003 \mathrm{~m}^{2}$ \\
\hline Linear density & $525.64 \mathrm{~kg} / \mathrm{m}$ \\
\hline Young modulus & $210 \mathrm{GPa}$ \\
\hline
\end{tabular}

\subsection{PTO optimization in irregular waves}

In order to optimize the device performance under the sea conditions defined in the previous section, different values of $\mathrm{K}_{\mathrm{PTO}}$ have been considered. Simulations have been carried out for 1 hour, where average power production has converged.

Table 47 provides the average power production for each value of the PTO, and Figure 89 shows it in a graph. For $\mathrm{K}_{\mathrm{PTO}}$ around $5 \times 10^{7}$ the average power production reaches the maximum value of 26 kw.

Figure 90 shows the instantaneous moment and power, and average power. Figure 91 shows the instantaneous surge, heave, and pitch movements of the device for the specific case $K_{\text {РтO }}=$ $50 \mathrm{MNm} /(\mathrm{rad} / \mathrm{s})$. In Figure 92, a snapshot of the device, including the mooring lines and free surface elevation, can be observed. 


\subsection{Summary}

A Pelamis type device has been analyzed to show the capabilities of the solver to simulate a multibody system with body links. The device consists of two segments coupled by means of a revolute joint (hinge type), and with a simple PTO system that works as a relative pitch damper between segments. Simulations have been carried out to show how to tune PTO parameters for which the maximum average power under given environmental conditions is produced.

Table 47: Average power generated versus PTO arrangement

\begin{tabular}{|c|c|c|c|}
\hline $\boldsymbol{K}_{\text {PTO }}\left(\frac{\boldsymbol{M N} \cdot \boldsymbol{m}}{\boldsymbol{r a d} / \boldsymbol{s}}\right)$ & $\boldsymbol{P}_{\text {average }}(\boldsymbol{K w})$ & $\boldsymbol{K}_{\text {PTo }}\left(\frac{\boldsymbol{M N} \cdot \boldsymbol{m}}{\boldsymbol{r a d} / \boldsymbol{s}}\right)$ & $\boldsymbol{P}_{\text {average }}(\boldsymbol{K w})$ \\
\hline 5 & 7.25 & 100 & 23.2 \\
\hline 10 & 12.7 & 200 & 15.9 \\
\hline 20 & 19.7 & 500 & 7.37 \\
\hline 50 & 26.1 & 1000 & 3.80 \\
\hline
\end{tabular}

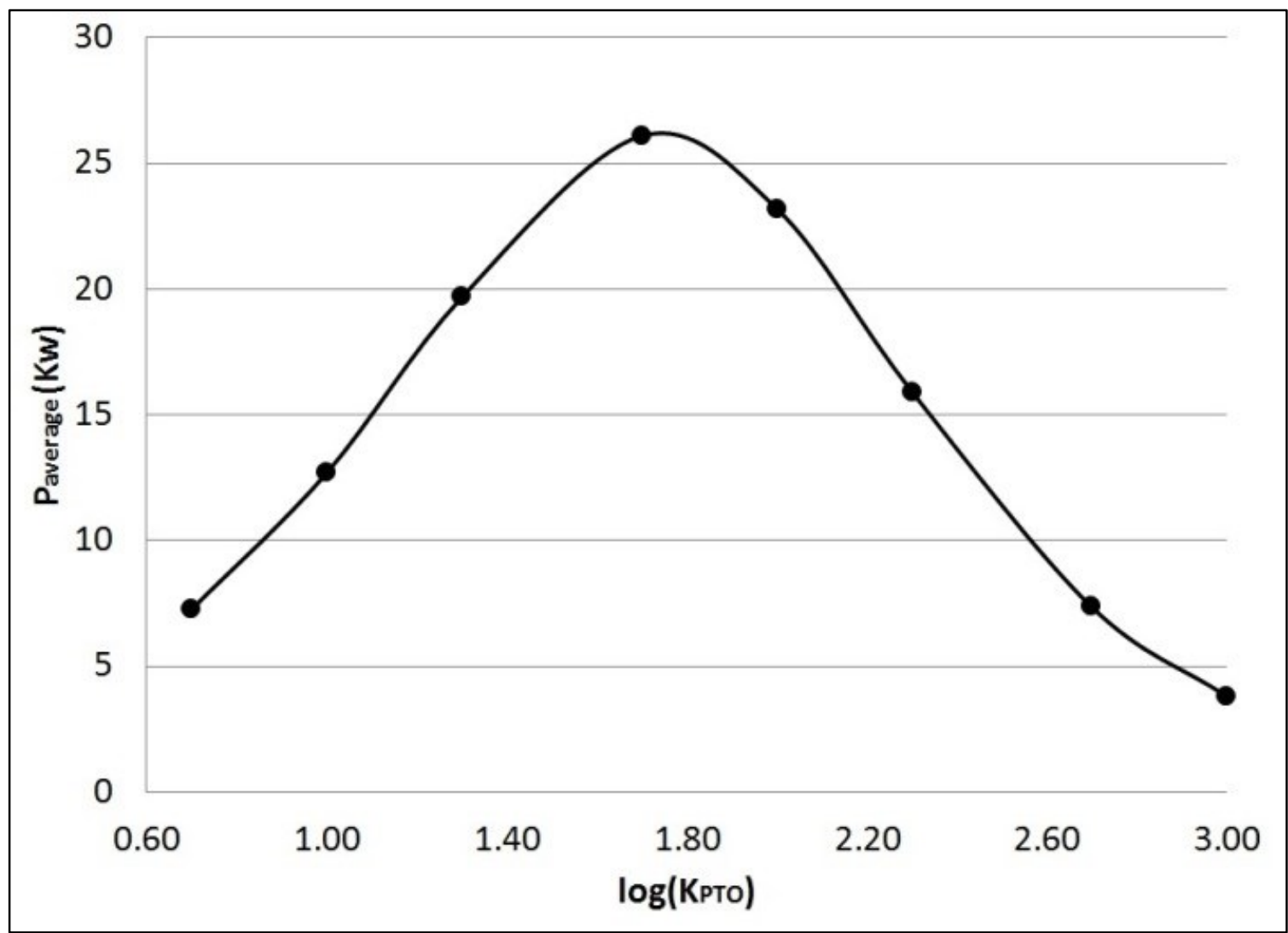

Figure 89: Average power production versus $\log \left(\mathrm{K}_{\mathrm{PTO}}\right)$. 


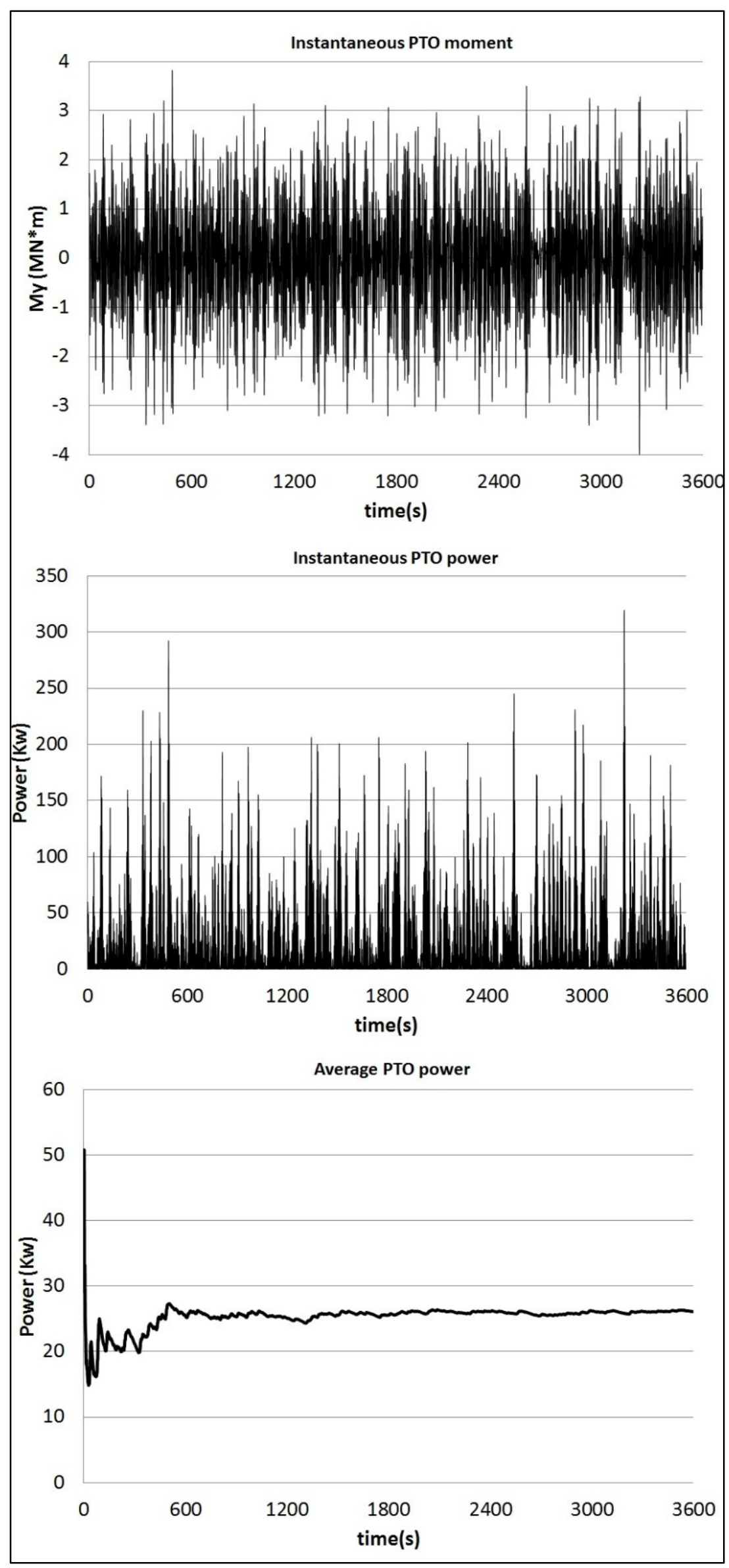

Figure 90: PTO data for $\mathrm{K}_{\mathrm{PTO}}=5 \cdot 10^{7} \mathrm{Nm} /(\mathrm{rad} / \mathrm{s})$. 


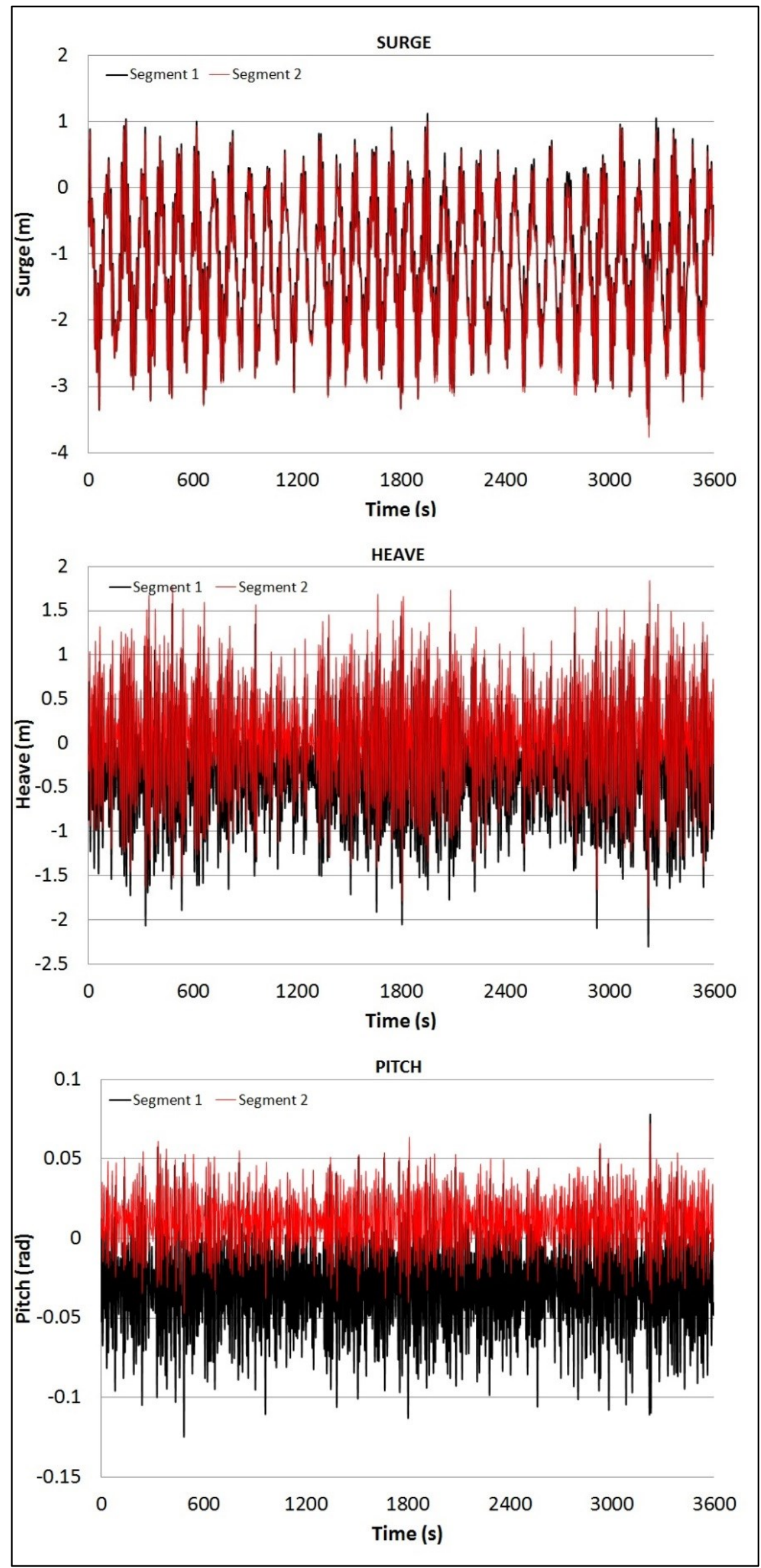

Figure 91: Surge, heave, and pitch movement of the device for $\mathrm{K}_{\mathrm{PTO}}=5 \cdot 10^{7} \mathrm{Nm} /(\mathrm{rad} / \mathrm{s})$. 


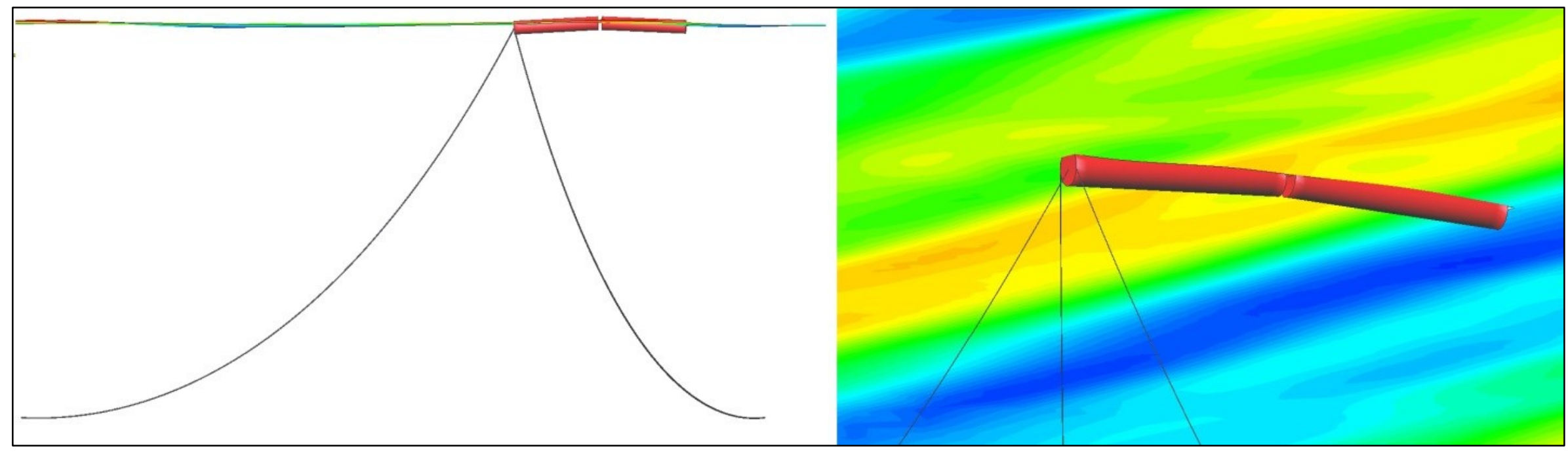

Figure 92: Snapshots of the device for $\mathrm{K}_{\mathrm{PTO}}=5 \cdot 10^{7} \mathrm{Nm} /(\mathrm{rad} / \mathrm{s})$. 


\section{Chapter 14. CONCLUSIONS}

\subsection{Conclusions}

The objective of this thesis has been to develop numerical algorithms and implement them as a numerical tool to simulate seakeeping behavior and wave resistance of ships and floating structures. The first tool developed is a wave diffraction-radiation solver. It is based on the finite element method in order to solve the fluid flow model, governed by the Laplace equation. Two different cases are considered depending on whether or not convective terms in the free surface boundary conditions are dominant.

When convective terms are second order, a mathematical model for the seakeeping problems is presented in Chapter 2. It has been found that a fourth-order Padé scheme performs well to solve the free surface boundary condition with uniform and constant free surface pressure. An extension of the latter scheme has been provided to take into account free surface pressure variations. A number of verification and validation cases have been carried out in Chapter 5, as well as inter-code comparison with other methods. The numerical results obtained in this thesis compare well with the analytical, experimental, and numerical results obtained by other methods.

A mathematical model for the wave making resistance and added resistance in waves when convective terms are of first order has been presented in Chapter 3. Two different numerical schemes have been proved to perform well to solve the free surface condition with first-order convective terms: the FEM-SUPG and the streamline integration scheme. Moreover, special attention is given to the second order terms depending on the first-order solution since they are responsible for the added resistance in waves. Verification and validation cases have been carried out in Chapter 6. The numerical results obtained in this thesis compare well with analytical and experimental ones. In the experimental comparisons, due to the large scattering found in the experimental data, it is difficult to find a fair fitting, but trends are well captured.

In 0 the mathematical and numerical models to solve rigid body dynamics of multibody systems with body links has been presented. This tool has been integrated along with the wave diffractionradiation solver, so that multi-body systems interacting with waves can be solved. The rigid body dynamics solver is used in most verification and validation cases across the thesis. In particular, in Chapter 7, a verification case for the body links, based on a rotating ring, is analyzed. The results show a good performance of the solver with body links. 
Solver acceleration is another topic considered in this thesis. Most of the CPU time required by the developed tools is spent in solving the linear system of equations resulting from the discretization of the fluid domain to solve the Laplace equations. Two techniques have been considered to this purpose: solver deflation and the use of graphic processing units (GPUs).

Regarding solver deflation, a method for domain decomposition required by deflated solvers has been proposed. Such a method is capable of effectively reducing solver iteration, and the larger the system of equations, the larger the reduction that can be achieved. However, a reduction in the number of iterations does not always lead to a reduction of CPU time. Therefore care must be taken when using a deflated solver.

It has been proven that the use of GPUs can speed up the wave diffraction-radiation solver, and comparisons against CPU have been performed for a number of cases using different preconditioners and architectures. In general, it has been found that using the GPU is faster that using a serial execution in a CPU for similar software generations.

In Chapter 9 coupling algorithms with other numerical tools in order to solve multi-physics problems have been tailored. In particular the coupling with a MEF structural solver to solve fluidstructure interaction problems. The dynamics of the interaction between the free surface and the seals of a surface effect ship has been analyzed. For this sort of analysis, a fluid-structure coupling algorithm has been developed, as well as an algorithm for limiting the free surface elevation by a solid surface. Comparison with experimental results shows a good agreement in the analysis of the seal deformation of a SES.

In 0 , a coupling strategy with a solver capable of simulating internal flows in tanks has been developed. The objective is to solve couple seakeeping-sloshing problems. Several validations cases have been simulated. Results show good agreement in general with the experimental results.

A mooring solver has been coupled with the tools developed in this thesis to carry out numerical simulations of a floating offshore wind turbines. A coupling algorithm has been designed and explained in detail to include mooring in Chapter 11. It has been used to analyze the Hywind (the OC3 offshore spar buoy wind turbine). For this case study, second order simulations have been performed. Aerodynamic loads have been considered for a fully coupled simulation. As a result, movements of the whole system and mooring lines loads have been obtained.

Finally Chapter 12 and Chapter 13 show two demonstration cases on how to use the developed tools to analyze two different wave energy converters. The first one is based on the oscillating water column principle. In this case, variations of pressure on the free surface have to be taken into account to be able to simulate the interaction between the power take off system and the free surface. As a result, the pressure inside the chamber, and the instantaneous and average power take off can be 
obtained. The second device is a Pelamis type device, where wave energy is extracted by means of the relative movements between two floating bodies. These bodies are connected by a mechanical system that imposes kinematic links between the two bodies. An optimization of this mechanical system has been performed in order to maximize the power extraction in one case scenario.

\section{$14.2 \quad$ Future work}

The author is currently one of the principal researchers of the X-SHEAKS project, focussing on the development of a mixed particle and FEM formulation tailored for seakeeping problems. This new method is based on a mixed Lagrangian-Eulerian formulation, where convective terms are solved via the Lagrangian formulation using particles, and the non-convective terms are solve via classical FE formulation. This new method is expected to be able to handle highly non-linear free surface problems such as wave breaking, sloshing, spray formation in high speed crafts, etc.

$\mathrm{X}$-SHEAKS is a collaborative project between CIMNE and ETSIN supported by the "Ministerio de Economía y Competitividad", project ENE2014-59194-C2-1-R.

\subsection{Publications from this thesis}

\subsubsection{Refereed papers}

- B. Serván-Camas and J. García-Espinosa (2013). Accelerated 3D multi-body seakeeping simulations using unstructured finite elements. Journal of Computational Physics 252, 382403.

- J. García-Espinosa, D. Di Capua, B. Serván-Camas, P.-A. Ubach, E. Oñate (2015). A FEM fluid-structure interaction algorithm for analysis of the seal dynamics of a Surface-Effect Ship. Computers Methods in Applied Mechanics and Engineering 295, 290-304.

\subsubsection{Submitted papers}

- B. Serván-Camas, J. L. Cercós-Pita, J. Colom-Cobb, J. García-Espinosa, Antonio SoutoIglesias (2015). Time domain simulation of coupled sloshing-seakeeping problems by SPHFEM coupling. Submitted to Ocean Engineering. 
- J. E. Gutiérrez-Romero, J. García-Espinosa, B. Serván-Camas, B. Zamora-Parra (2015). Nonlinear dynamic analysis of the response of moored floating structures. Submitted to Ocean Engineering.

\subsubsection{Conference papers}

- J.L. Cercos-Pita, A. Souto-Iglesias, G. Bulian, B. Bouscasse, A. Colagrossi, J. Colom, B. Serván, J. Garcia-Espinosa (2015). Coupling between sloshing and ship motions with emphasis on wave-breaking. The 16th International Workshop on Trends In Numerical and Physical Modeling for Industrial Multiphase Flows. September 14th-18th, 2015, Cargèse, Corsica.

- B. Serván-Camas, J.L. Cercós-Pita, J. Colom-Cobb, J. García-Espinosa (2015). Time-domain simulation of coupled sloshing-seakeeping problems by means of SPH-FEM coupling. VI International Conference on Computational Methods in Marine Engineering. Marine 2015. 1517 June 2015, Rome, Italy.

- J. García-Espinosa, P. Dinoi, C. López-Pavón, B. Serván-Camas (2015). Validation of mean drift loads induced on a semisubmersible offshore platform. VI International Conference on Computational Methods in Marine Engineering. Marine 2015. 15-17 June 2015, Rome, Italy.

- J. García-Espinosa, B. Serván-Camas, D. Di Capua, P. Ubach, E. Oñate (2013). Coupled wavestructure analysis for naval and offshore applications. V International Conference on Computational Methods in Marine Engineering. Marine 2013. 29-31 May 2013, Hamburg, Germany.

- J. García-Espinosa and B. Serván-Camas (2013). A Time-Domain Multi-Body Finite Element Method for Evaluation of Ocean Energy Converters. V International Conference on Computational Methods in Marine Engineering. Marine 2013. 29-31 May 2013, Hamburg, Germany.

- J. García-Espinosa, B. Serván-Camas, D. Di Capua, P.A. Ubach, E. Oñate (2013). Evaluating Performance of the Air Cushion, and Seals of a SES (Surface-Effect Ship) in the Presence of Waves, and Maneuvering in Shallow Waters. V International Conference on Computational Methods in Marine Engineering. Marine 2013. 29-31 May 2013, Hamburg, Germany. 
- D. Julio García, D. Borja Serván, D. Daniel Di Capua, D. Eugenio Oñate (2013). Advances in the development of a FEM model for seakeeping and maneuvering evaluation of a T-Craft including skirt dynamics. $52^{\circ}$ Congreso de Inigeniería Naval e Industria Marítima. 23-25 ctubre 2013, Madrid.

- B. Serván-Camas and J. García-Espinosa (2011). Advances in the Development of a Timedomain Unstructured Finite Element Method for the Analysis of Waves and Floating Structures Interaction. V International Conference on Computational Methods in Marine Engineering. Marine 2011. 28-30 September 2011, Lisbon, Portugal.

\subsection{SeaFEM}

The author of this thesis is the main developer of SeaFEM [132], a seakeeping software developed at the International Center for Numerical Methods in Engineering (CIMNE) in collaboration with CompassIS. SeaFEM is a suite of tools for seakeeping and wave resistance problems, including a wavediffraction radiation solver, a body dynamics solver capable of including kinematic constraints (body joints), and a mooring solver, as well as utilities to define mooring systems and Morison elements. The first and second order wave diffraction-radiation solver, body dynamics solver (including body links), acceleration techniques, coupling strategies, and wave spectra discretization implemented in SeaFEM are based on the work presented in this thesis. 
APPENDICES 


\section{APPENDIX A. STATISTICAL DESCRIPTION OF WAVES}

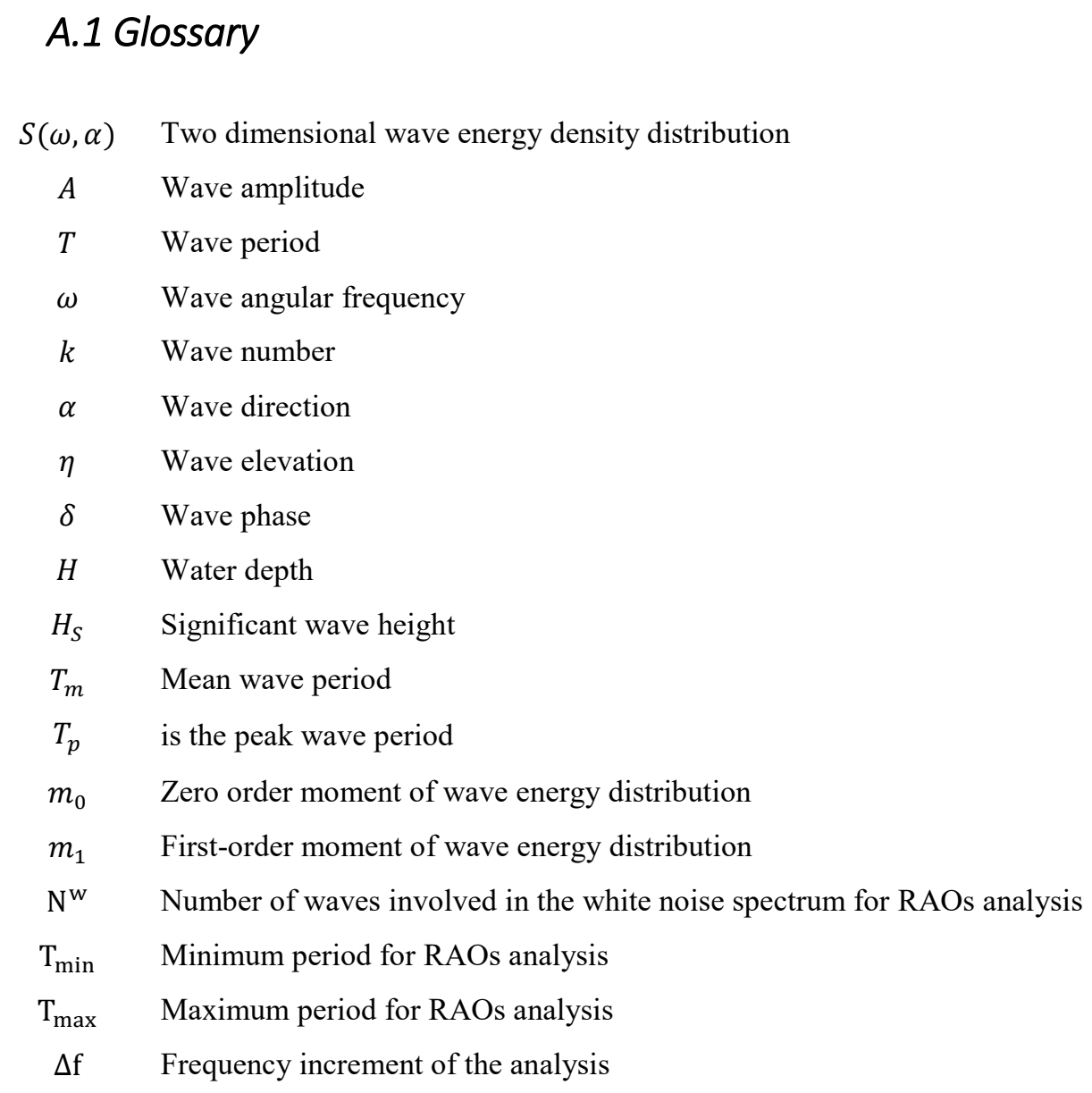

\section{A.2 Introduction}

This chapter aims at explaining the wave spectra used in this thesis to represent irregular seas, as well as the discretization used for that purpose. Moreover, it is explained how response amplitude operators can be obtained by Fourier analysis using irregular waves.

\section{A.3 Spectrum discretization}

Let $S(\omega, \alpha)$ be an energy density spectrum describing a sea state in terms of the wave frequency and direction of propagation. The discretization procedure to obtain a stationary and ergodic realization based on monochromatic waves is as follows: 
Let $\omega_{\min }$ be the minimum frequency to be considered, $\omega_{\max }$ the maximum frequency to be considered, $\alpha_{\min }$ the lower direction of propagation to be considered, $\alpha_{\max }$ the larger direction of propagation to be considered, $N^{\omega}$ the number of wave frequencies, and $N^{\alpha}$ the number of wave directions to be considered. Then, the frequency and direction discretization sizes are given by:

$$
\begin{aligned}
& \Delta \omega=\frac{\left(\omega_{\max }-\omega_{\min }\right)}{N^{w}}, \\
& \Delta \alpha=\frac{\left(\alpha_{\max }-\alpha_{\min }\right)}{\left(N^{\alpha}-1\right)},
\end{aligned}
$$

and the wave elevation is given by:

$$
\eta=\sum_{i=1}^{N^{w}} \sum_{j=1}^{N^{\alpha}} A_{i j} \cos \left(k_{i j} \cos \left(\alpha_{j}\right) x+k_{i j} \sin \left(\alpha_{j}\right)-\Omega_{i j} t+\delta_{i j}\right),
$$

where $\Omega_{i j}$ is the wave angular velocity and a random variable with uniform distribution within [ $\omega_{i}-$ $\left.\frac{\Delta \omega}{2}, \omega_{i}+\frac{\Delta \omega}{2}\right], \omega_{i}=\omega_{\min }+(i-1 / 2) \Delta \omega, \alpha_{j}=\alpha_{\min }+(j-1) \Delta \alpha, \delta_{i j}$ is a random variable with uniform distribution in $[0,2 \pi], t$ represents time, and $\mathrm{x}, \mathrm{y}$ are the horizontal Cartesian coordinates. The wave number is obtained from the dispersion relationship:

$$
\Omega^{2}{ }_{i j}=g k_{i j} \tanh \left(k_{i j} H\right)
$$

and the wave amplitude is calculated from the wave energy distribution as:

$$
\mathrm{A}_{i j}=\sqrt{2 \Delta \omega \Delta \alpha S\left(\omega_{i}, \alpha_{j}\right)} \frac{\frac{1}{16} H_{S}^{2}}{\sum_{l, m} \sqrt{2 \Delta \omega \Delta \alpha S\left(\omega_{l}, \alpha_{m}\right)}},
$$

where $H_{S}=4 \sqrt{m_{0}}$ is the significant wave height, and $m_{0}=\int_{0}^{\infty} \int_{-\pi}^{\pi} S(\omega, \alpha) d \omega d \alpha$ is zero order moment of the spectrum wave energy.

\section{A.4 Convergence}

Convergence of the discretized spectrum will happen as $\omega_{\min } \rightarrow 0, \omega_{\max } \rightarrow \infty, \Delta \omega \rightarrow 0, \alpha_{\text {min }} \rightarrow$ $-\pi, \alpha_{\max } \rightarrow \pi$, and $\Delta \alpha \rightarrow 0$. The rate of convergence with $\Delta \omega$ and $\Delta \alpha$ is that of the rectangle rule of numerical integration.

\section{A.5 Spectral moments}

\section{A.5.1 Zero order moment}

The spectral energy of a wave spectrum is given by: $m_{0}=\int_{0}^{\infty} \int_{-\pi}^{\pi} S(\omega, \alpha) d \omega d \alpha=\frac{1}{16} \rho g H_{S}^{2}$. Then, the discrete spectrum is scaled such that the spectral moment $m_{0}$ is conserved. Therefore: 
$\sum_{i, j} \frac{1}{2} A_{i j}^{2}=\frac{1}{16} H_{S}^{2}$

\section{A.5.2 First-order moment}

The first-order moment of the discrete spectrum is:

$$
m_{1}^{*}=\sum_{i, j} S\left(\omega_{i}, \alpha_{j}\right) \Omega_{i, j} \Delta \omega \Delta \alpha=\sum_{i, j} S\left(\omega_{i}, \alpha_{j}\right) \omega_{i, j} \Delta \omega \Delta \alpha+\sum_{i, j} S\left(\omega_{i}, \alpha_{j}\right) \epsilon_{i, j} \Delta \omega \Delta \alpha,
$$

where $\epsilon_{i, j}$ is uniform distributed between $\left[-\frac{\Delta \omega}{2}, \frac{\Delta \omega}{2}\right], \sum_{i, j} S\left(\omega_{i}, \alpha_{j}\right) \Omega_{i, j} \Delta \omega \Delta \alpha$ is a deterministic component of the first moment, and $\sum_{i, j} S\left(\omega_{i}, \alpha_{j}\right) \epsilon_{i, j} \Delta \omega \Delta \alpha$ is a random component. Assuming that $\omega_{\max } \rightarrow \infty, \omega_{\min }=0, \alpha_{\min }=-\pi, \alpha_{\max }=\pi$, the deterministic component converges to:

$$
\lim _{\substack{\Delta \omega \rightarrow 0 \\ \Delta \alpha \rightarrow 0}} \sum_{i, j} S\left(\omega_{i}, \alpha_{j}\right) \omega_{i, j} \Delta \omega \Delta \alpha=\int_{0}^{\infty} \int_{-\pi}^{\pi} \omega S(\omega, \alpha) d \omega d \alpha
$$

On the other hand, for large values of $N^{\omega}$, the probabilistic component is a random variable with normal distribution. Based on the central limit theorem, the mean $\mu$ and variance $\sigma^{2}$ of this distribution are:

$$
\begin{aligned}
& \mu=\sum_{i, j} S\left(\omega_{i}, \alpha_{j}\right) \Delta \omega \Delta \alpha \int_{-\Delta \omega / 2}^{\Delta \omega / 2} \omega \frac{1}{\Delta \omega} d \omega, \\
& \sigma^{2}=\sum_{i, j} S\left(\omega_{i}, \alpha_{j}\right) \Delta \omega \Delta \alpha \int_{-\Delta \omega / 2}^{\Delta \omega / 2} \omega^{2} \frac{1}{\Delta \omega} d \omega=\sum_{i, j} S\left(\omega_{i}, \alpha_{j}\right) \Delta \omega \Delta \alpha \frac{\Delta \omega^{2}}{12} .
\end{aligned}
$$

The probabilistic component converges to a random variable with zero mean and zero variance.

\section{A.6 Wave spectrums}

\section{A.6.1 Pearson-Moskowitz}

The Pearson-Moskowitz spectrum was obtained by assuming a fully developed sea state, generated by wind blowing steadily for a long time over a large area [133]. The resulting spectrum was [134]:

$$
S(T)=H_{s}^{2} T_{m}\left(\frac{0.11}{2 \pi}\right)\left(\frac{T_{m}}{T}\right)^{-5} e^{-0.44\left(\frac{T m}{T}\right)^{-4}},
$$

where $T_{m}=2 \pi m_{0} / m_{1}$, with $m_{0}$ and $m_{1}$ the zero and first moments of the wave spectrum. 


\section{A.6.2 Jonswap}

The "JOint North Sea WAve Project" (JONSWAP) spectrum was established during a joint research project [135]. This is a peak-enhanced Pierson-Moskowitz spectrum with the following expression [42]:

$$
\begin{aligned}
& S(T)=\left(\frac{5}{32 \pi} H_{S}^{2} \frac{\mathrm{T}^{5}}{\mathrm{~T}_{\mathrm{p}}^{4}}\right) \cdot \varepsilon^{\gamma} \cdot \mathrm{e}^{-1.25\left({ }^{\mathrm{T}} \mathrm{p} / \mathrm{T}\right)^{-4}(1-0.287 \log (\epsilon))}, \\
& \gamma=\mathrm{e}^{-\left[\left(0.159 \omega \mathrm{T}_{\mathrm{p}}^{-1}\right) /(\sigma \sqrt{2})\right]^{2}},
\end{aligned}
$$

where $\omega=2 \pi / T, \sigma=0.07$ for $\omega \leq 6.28 / T_{p}, \sigma=0.09$ for $\omega>6.28 / T_{p}, T$ is the wave period; $H_{s}$ is the significant wave height, $\mathrm{T}_{\mathrm{p}}$ is the peak wave period and $\epsilon$ is the peakedness parameter.

An alternative definition of the JONSWAP spectrum is given by [134]:

$$
\begin{aligned}
& S(\omega)=\left(\frac{155 H_{s}^{2}}{\mathrm{~T}_{\mathrm{m}}^{4} \omega^{5}}\right) \cdot 3.3^{\gamma} \cdot \mathrm{e}^{-944 \mathrm{~T}_{\mathrm{m}}^{-4} \omega^{-4}}, \\
& \gamma=\mathrm{e}^{-\left[\left(0.191 \omega \mathrm{T}_{\mathrm{m}}^{-1}\right) /(\sigma \sqrt{2})\right]^{2}},
\end{aligned}
$$

where $\sigma=0.07$ for $\omega \leq 5.24 / T_{m}, \sigma=0.09$ for $\omega>5.24 / T_{m}, T_{m}=2 \pi m_{0} / m_{1}$.

\section{A.6.3 White noise}

The white noise spectrum corresponds to an uniform energy distribution within a wave frequency interval, having zero energy outside the prescribed interval. This type of spectrum is used in this thesis to carry out response amplitude operators (RAOs) analyses.

\section{A.7 Response amplitude operators (RAOs)}

RAOs are transfer functions of the ratio between the incident wave amplitudes and body movements, used to determine the effect that a sea state will have upon the motion of a floating body. Calculations of RAOs are carried out by analyzing the time series response of the ship, using a discretized white noise spectrum. This spectrum is defined by a number $\mathrm{N}^{\mathrm{w}}=2^{\mathrm{m}-1}$ of waves of equal amplitude and periods varying between the maximum and minimum values to be defined. The values of these $\mathrm{N}^{\mathrm{w}}$ periods are selected to match the discrete Fourier transform of the output signal, given by: $X=\sum_{n=0}^{N^{W}-1} x_{n} \cdot e^{-i 2 \pi \Delta f^{*} n}$

Given the minimum $\left(\mathrm{T}_{\min }\right)$ and maximum $\left(\mathrm{T}_{\max }\right)$ periods for the analysis, the wave frequency discrete increment to be used is: 


$$
\Delta \mathrm{f}=\left(\mathrm{f}_{\max }-\mathrm{f}_{\min }\right) /\left(\mathrm{N}^{\mathrm{w}}-1\right)=\left(\frac{1}{\mathrm{~T}_{\min }}-\frac{1}{\mathrm{~T}_{\max }}\right) /\left(\mathrm{N}^{\mathrm{w}}-1\right) .
$$

The well-known Fast Fourier Transform algorithms give a procedure to obtain an exact evaluation of the transfer functions defined above. This way, the time step and the total computing time can be fixed to match the required sampling time and total sampling points. Then, the holding frequency $\Delta \mathrm{f}^{*}$ is evaluated as

$$
\Delta \mathrm{f}^{*}=\min \left(\Delta \mathrm{f}, \mathrm{f}_{\min }\right) \text {, }
$$

and the discrete frequencies are:

$$
\mathrm{f}_{\mathrm{n}}=\mathrm{n} \cdot \Delta \mathrm{f} \quad \mathrm{n}=0,1,2, \ldots, \mathrm{N}^{\mathrm{w}}-1 .
$$

The required sampling frequency defines the time step as:

$$
\Delta \mathrm{t}=\frac{1}{2 \Delta \mathrm{f}^{*} \cdot 2^{\mathrm{m}}}
$$

and the required number of sampling points defines the total calculation time as $T=1 / \Delta \mathrm{f}^{*}$. 


\section{APPENDIX B. SECOND ORDER WAVES}

\section{B.1 First-order waves}

The first-order wave problem is governed by the following governing equations:
$\Delta \psi^{1}=0$
in $\Omega$,
fluid equation,
$\frac{\partial \psi^{1}}{\partial \mathrm{t}}+g \zeta^{1}=0 \quad$ on $z=0$,
dynamic free surface boundary condition,
$\frac{\partial \zeta^{1}}{\partial \mathrm{t}}-\frac{\partial \psi^{1}}{\partial \mathrm{z}}=0 \quad$ on $z=0$
kinematic free surface boundary condition,
$\frac{\partial \psi^{1}}{\partial \mathrm{z}}=0$
on $z=-H$,
bottom boundary condition,

where $\psi^{1}$ is the first-order velocity potential, $\zeta^{1}$ is the first-order free surface elevation, and $\mathrm{H}$ is the water depth.

The dynamic and kinematic boundary conditions can be combined into one boundary equation, decoupling the free surface elevation from solving the velocity potential.

$\frac{\partial^{2} \psi^{1}}{\partial \mathrm{t}^{2}}+g \frac{\partial \psi^{1}}{\partial \mathrm{z}}=0 \quad$ on $z=0, \quad$ Free surface boundary condition.

And the solution to the above stated problem is:

$$
\begin{aligned}
\psi^{1} & =\sum_{m} \frac{A_{m} g}{\omega_{m}} \frac{\cosh \left(\left|\boldsymbol{k}_{m}\right|(H+z)\right)}{\cosh \left(\left|\boldsymbol{k}_{m}\right| H\right)} \sin \left(\theta_{m}\right), \\
\zeta^{1} & =\sum_{i} A_{m} \cos \left(\theta_{m}\right),
\end{aligned}
$$

where $A_{m}$ is the wave amplitude, $\omega_{m}$ is the wave frequency, $\boldsymbol{k}_{m}$ is the wave number, $\theta_{m}=\boldsymbol{k}_{m} \boldsymbol{x}-$ $\omega_{m} t+\delta_{m}$ is the wave phase, and $\delta_{m}$ is the phase delay.

\section{B.2 Second-order waves}

The second-order wave problem is governed by the following governing equations:

$$
\begin{array}{ll}
\Delta \psi^{2}=0 & \text { in } \Omega, \\
\frac{1}{g} \frac{\partial \psi^{2}}{\partial \mathrm{t}}+\zeta^{2}=-\frac{1}{g} \zeta^{1} \frac{\partial}{\partial z}\left(\frac{\partial \psi^{1}}{\partial t}\right)-\frac{1}{2 g} \nabla \psi^{1} \cdot \nabla \psi^{1}=F^{1} & \text { on } z=0, \\
\frac{\partial \zeta^{2}}{\partial \mathrm{t}}-\frac{\partial \psi^{2}}{\partial \mathrm{z}}=\zeta^{1} \frac{\partial^{2} \psi^{1}}{\partial z^{2}}-\frac{\partial \zeta^{1}}{\partial x} \frac{\partial \psi^{1}}{\partial x}-\frac{\partial \zeta^{1}}{\partial y} \frac{\partial \psi^{1}}{\partial y}=G^{1} & \text { on } z=0,
\end{array}
$$


$\frac{\partial \psi^{2}}{\partial \mathrm{z}}=0$

$$
\text { on } z=-H \text {, }
$$

where $\psi^{2}$ is the second-order velocity potential, $\zeta^{2}$ is the second-order free surface elevation, and $F^{1}$ and $G^{1}$ are terms depending on the first-order solution.

$$
\begin{aligned}
F^{1} & =-\frac{1}{g}\left(\zeta^{1} \frac{\partial^{2} \psi^{1}}{\partial z \partial t}\right)-\frac{1}{2 g}\left(\nabla \psi^{1} \cdot \nabla \psi^{1}\right), \\
G^{1} & =\zeta^{1} \frac{\partial^{2} \psi^{1}}{\partial z^{2}}-\nabla_{h} \zeta^{1} \cdot \nabla_{h} \psi^{1} .
\end{aligned}
$$

Combining Eqs. (B-9) and (B-10):

$$
\frac{1}{g} \frac{\partial^{2} \psi^{2}}{\partial \mathrm{t}^{2}}+\frac{\partial \psi^{2}}{\partial \mathrm{z}}=\frac{\partial F^{1}}{\partial t}-G^{1} \quad \text { on } z=0 .
$$

\section{B.2.1 Inhomogeneous part}

After inserting Eqs. (B-6) and (B-7) into the inhomogenous part of (B-14), the latter can be decomposed as follows:

$$
\frac{\partial F^{1}}{\partial t}-G^{1}=\sum_{m} \sum_{n}\left(\frac{\partial F_{m n}^{1}}{\partial t}-G_{m n}^{1}\right)
$$

where

$$
\begin{aligned}
& \frac{\partial F_{m n}^{1}}{\partial t}=-\frac{1}{g} \frac{\partial}{\partial t}\left(\zeta_{m}^{1} \frac{\partial^{2} \psi_{n}^{1}}{\partial z \partial t}\right)-\frac{1}{2 g} \frac{\partial}{\partial t}\left(\nabla \psi_{\mathrm{m}}^{1} \cdot \nabla \psi_{n}^{1}\right), \\
& -G_{m n}^{1}=-\zeta_{m}^{1} \frac{\partial^{2} \psi_{n}^{1}}{\partial z^{2}}+\nabla_{h} \zeta_{m}^{1} \cdot \nabla_{h} \psi_{n}^{1} .
\end{aligned}
$$

\section{B.2.1.1 Inhomogeneous term 1}

$$
-\frac{1}{g} \frac{\partial}{\partial t}\left(\zeta_{m}^{1} \frac{\partial^{2} \psi_{n}^{1}}{\partial z \partial t}\right)=C_{m n 1}^{+} \sin \left(\theta_{m}+\theta_{n}\right)+C_{m n 1}^{-} \sin \left(\theta_{m}-\theta_{n}\right) \text {. }
$$

Introducing Eq. (B-6) and (B-7) into Eq. (B-18), and after some algebra:

$$
\begin{aligned}
& C_{m n 1}^{+}=\frac{A_{m} A_{n}}{2} \frac{g\left|\boldsymbol{k}_{n}\right|^{2} \tanh ^{2}\left(\left|\boldsymbol{k}_{n}\right| H\right)}{\omega_{n}^{2}}\left(\omega_{m}+\omega_{n}\right), \\
& C_{m n 1}^{-}=\frac{A_{m} A_{n}}{2} \frac{g\left|k_{n}\right|^{2} \tanh ^{2}\left(\left|k_{n}\right| H\right)}{\omega_{n}^{2}}\left(\omega_{m}-\omega_{n}\right) .
\end{aligned}
$$

Using the first-order dispersion relation:

$$
C_{m n 1}^{+}=\frac{A_{m} A_{n}}{2 g} \omega_{n}^{2}\left(\omega_{m}+\omega_{n}\right) \text {, }
$$


$C_{m n 1}^{-}=\frac{A_{m} A_{n}}{2 g} \omega_{n}^{2}\left(\omega_{m}-\omega_{n}\right)$

\section{B.2.1.2 Inhomogeneous term 2}

$-\frac{1}{2 g} \frac{\partial}{\partial t}\left(\nabla \psi_{\mathrm{m}}^{1} \cdot \nabla \psi_{n}^{1}\right)=C_{m n 2}^{+} \sin \left(\theta_{m}+\theta_{n}\right)+C_{m n 2}^{-} \sin \left(\theta_{m}-\theta_{n}\right)$.

Introducing Eqs. (B-6) and (B-7) into Eq. (B-23), and after some algebra:

$C_{m n 2}^{+}=\frac{A_{m} A_{n}}{4} \frac{g\left(\omega_{m}+\omega_{n}\right)}{\omega_{m} \omega_{n}}\left(\left|\boldsymbol{k}_{m}\right|\left|\boldsymbol{k}_{n}\right| \tanh \left(\left|\boldsymbol{k}_{m}\right| H\right) \tanh \left(\left|\boldsymbol{k}_{n}\right| H\right)-\boldsymbol{k}_{m} \cdot \boldsymbol{k}_{n}\right)$,
$C_{m n 2}^{-}=\frac{A_{m} A_{n}}{4} \frac{g\left(\omega_{m}-\omega_{n}\right)}{\omega_{m} \omega_{n}}\left(\left|\boldsymbol{k}_{m}\right|\left|\boldsymbol{k}_{n}\right| \tanh \left(\left|\boldsymbol{k}_{m}\right| H\right) \tanh \left(\left|\boldsymbol{k}_{n}\right| H\right)+\boldsymbol{k}_{m} \cdot \boldsymbol{k}_{n}\right)$.

Using the first-order dispersion relation:

$C_{m n 2}^{+}=\frac{A_{m} A_{n}}{4 g}\left(\omega_{m} \omega_{n}-g^{2} \frac{\boldsymbol{k}_{m} \cdot \boldsymbol{k}_{n}}{\omega_{m} \omega_{n}}\right)\left(\omega_{m}+\omega_{n}\right)$,
$C_{m n 2}^{-}=\frac{A_{m} A_{n}}{4 g}\left(\omega_{m} \omega_{n}+g^{2} \frac{\boldsymbol{k}_{m} \cdot \boldsymbol{k}_{n}}{\omega_{m} \omega_{n}}\right)\left(\omega_{m}-\omega_{n}\right)$.

\section{B.2.1.3 Inhomogeneous term 3}

$-\zeta_{m}^{1} \frac{\partial^{2} \psi_{n}^{1}}{\partial z^{2}}+\nabla_{h} \zeta_{m}^{1} \cdot \nabla_{h} \psi_{n}^{1}=C_{m n 3}^{+} \sin \left(\theta_{m}+\theta_{n}\right)+C_{m n 3}^{-} \sin \left(\theta_{m}-\theta_{n}\right)$.

Introducing Eqs. (B-6) and (B-7) into Eq. (B-28), and after some algebra:

$$
\begin{aligned}
& C_{m n 3}^{+}=-\frac{A_{m} A_{n} g}{2} \frac{\left|\boldsymbol{k}_{n}\right|^{2}+\boldsymbol{k}_{m} \cdot \boldsymbol{k}_{n}}{\omega_{n}}, \\
& C_{m n 3}^{+}=+\frac{A_{m} A_{n} g}{2} \frac{\left|\boldsymbol{k}_{n}\right|^{2}-\boldsymbol{k}_{m} \cdot \boldsymbol{k}_{n}}{\omega_{n}} .
\end{aligned}
$$

\section{B.2.1.3 Inhomogeneous term}

The inhomogeneous term can be written in terms of the first-order components as:

$\frac{\partial F^{1}}{\partial t}-G^{1}=\sum_{m} \sum_{n}\left(\sum_{i=1}^{3} C_{m n i}^{+} \sin \left(\theta_{m}+\theta_{n}\right)+\sum_{i=1}^{3} C_{m n i}^{-} \sin \left(\theta_{m}-\theta_{n}\right)\right)$.

\section{B.2.2 Second-order velocity potential}

A second order solution can be expressed as:

$\psi^{2}=\sum_{m} \sum_{n} \psi_{m n}^{2}$ 
where each component $\psi_{m n}^{2}$ fulfills:

$$
\begin{array}{ll}
\Delta \psi_{m n}^{2}=0 & \text { in } \Omega, \\
\frac{1}{g} \frac{\partial^{2} \psi_{m n}^{2}}{\partial \mathrm{t}^{2}}+\frac{\partial \psi_{m n}^{2}}{\partial \mathrm{z}}=\frac{\partial F_{m n}^{1}}{\partial t}-G_{m n}^{1} & \text { on } z=0, \\
\frac{\partial \psi_{m n}^{2}}{\partial \mathrm{z}}=0 & \text { on } z=-H .
\end{array}
$$

The following solution for $\psi_{m n}^{2}$ fulfills the Laplace equation and the bottom boundary condition, and can recover the inhomogeneous part of the free surface condition:

$$
\begin{aligned}
\psi_{m n}^{2}=B_{m n}^{+} & \cosh \left(\left|\boldsymbol{k}_{m}+\boldsymbol{k}_{n}\right|(H+z)\right) \sin \left(\theta_{m}+\theta_{n}\right) \\
& +B_{m n}^{-} \cosh \left(\left|\boldsymbol{k}_{m}-\boldsymbol{k}_{n}\right|(H+z)\right) \sin \left(\theta_{m}-\theta_{n}\right) .
\end{aligned}
$$

Introducing Eq. (B-36) into Eq. (B-34) we obtain:

$$
\begin{aligned}
D_{m n}^{+} B_{m n}^{+} \sin \left(\theta_{m}+\theta_{n}\right)+D_{m n}^{-} B_{m n}^{-} \sin \left(\theta_{m}-\theta_{n}\right) \\
=\sum_{i=1}^{3}\left(C_{m n i}^{+} \sin \left(\theta_{m}+\theta_{n}\right)+C_{m n i}^{-} \sin \left(\theta_{m}-\theta_{n}\right)\right),
\end{aligned}
$$

where

$$
\begin{aligned}
& D_{m n}^{+}=-\frac{1}{g}\left(\omega_{m}+\omega_{n}\right)^{2} \cosh \left(\left|\boldsymbol{k}_{m}+\boldsymbol{k}_{n}\right| H\right)+\left|\boldsymbol{k}_{m}+\boldsymbol{k}_{n}\right| \sinh \left(\left|\boldsymbol{k}_{m}+\boldsymbol{k}_{n}\right| H\right), \\
& D_{m n}^{-}=-\frac{1}{g}\left(\omega_{m}-\omega_{n}\right)^{2} \cosh \left(\left|\boldsymbol{k}_{m}+\boldsymbol{k}_{n}\right| H\right)+\left|\boldsymbol{k}_{m}-\boldsymbol{k}_{n}\right| \sinh \left(\left|\boldsymbol{k}_{m}+\boldsymbol{k}_{n}\right| H\right),
\end{aligned}
$$

hence:

$$
\begin{aligned}
& B_{m n}^{+}=\sum_{i=1}^{3} C_{m n i}^{+} / D_{m n}^{+}, \\
& B_{m n}^{-}=\sum_{i=1}^{3} C_{m n i}^{-} / D_{m n}^{-} .
\end{aligned}
$$


[1] B. Borgarino, A. Babarit, P. Ferrant (2013). An Implementation of the Fast Multipole Algorithm for Wave Interaction Problems on Sparse Arrays of Floating Bodies, Journal of Engineering Mathematics, Vol.77, pp 51-68.

[2] R.Watai, P. Dinoi, F. Ruggeri, A. Souto-Iglesias, A.N. Simos (2015). Rankine time-domain method with application to side-by-side gap flow modeling. Applied Ocean Research, 50, 69-90.

[3] R. Watai (2014). A time-domain boundary elements method for the seakeeping analysis of offshore systems. Doctoral Thesis, Escola Politécnica da Universidade de Sao Paulo.

[4] X. Cai, H.P. Langtangen, B.F. Nielsen, and A. Tveito (1998). A finite element method for fully nonlinear water waves. J. Comput. Phys., 143, 544-568.

[5] G. Ducrozet, H.B. Bingham, A.P. Engsig-Karup, F. Bonnefoy, P. Ferrant (2012). A Comparative Study of Two Fast Nonlinear Free Surface Water Wave Models, Int. Journal for Numerical Methods in Fluids 69 (11), 1818-1834.

[6] E. Oñate and J. García (2001). A finite element method for fluid-structure interaction with surface waves using a finite calculus formulation. Comput. Methods Appl. Mech. Eng., 191, 635660 .

[7] R. Löhner, C. Yang, and E. Oñate (2006). On the simulation of flows with violent free surface motion. Comput. Methods Appl. Mech. Eng., 195.

[8] R. Löhner, C. Yang, and E. Oñate (2007). On the simulation of flows with violent free surface motion and moving objects using unstructured meshes. Comput. Methods Appl. Mech. Eng., 53, 1315-1338.

[9] J. García, A. Valls, and E. Oñate, ODDLS (2008). A new unstructured mesh finite element method for the analysis of free surface flow problems. Int. J. Numer. Methods Fluids, 76 (9), 12971327.

[10] G.G. Stokes (1847). On the theory of oscillatory waves. Trans. Camb. Philos. Soc., 8, 441-455.

[11] G.X. Wu and R. Eatock Taylor (1994). Finite element analysis of two-dimensional non-linear transient water waves. Appl. Ocean Res., 16, 363-372.

[12] G.X. Wu and R. Eatock Taylor (1995). Time stepping solution of the two-dimensional nonlinear wave radiation problem. Ocean Eng., 22, 785-798.

[13] D.M. Greaves, A.G.L. Borthwick, G.X. Wu, and R. Eatock Taylor (1997). A moving boundary finite element method for fully nonlinear wave simulation. J. Ship Res., 41, 181-194.

[14] I. Robertson and S. Sherwin (1999). Free-surface flow simulation using hp/spectral elements. J. Comput. Phys., 155, 26-53. 
[15] Q.W. Ma, G.X. Wu, and R. Eatock Taylor (2001). Finite element simulation of fully nonlinear interaction between vertical cylinders and steep waves - part 1: methodology and numerical procedure. Int. J. Numer. Methods Fluids, 36, 265-285.

[16] Q.W. Ma, G.X. Wu, and R. Eatock Taylor (2001). Finite element simulation of fully nonlinear interaction between vertical cylinders and steep waves - part 2: numerical results and validation. Int. J. Numer. Methods Fluids, 36, 287-308.

[17] J.H. Westhuis (2001). The numerical simulation of nonlinear waves in the hydrodynamic model test basin. Ph.D. thesis, Universiteit Twente, The Netherlands.

[18] P.X. Hu, G.X. Wu, and Q.W. Ma (2002). Numerical simulation of nonlinear wave radiation by a moving vertical cylinder. Ocean Eng., 29, 1733-1750.

[19] M.S. Turnbull, A.G.L. Borthwick, and R. Eatock Taylor (2003). Wave-structure intersection using coupled structured-unstructured finite element meshes. Appl. Ocean Res., 25, 63-77.

[20] G.X. Wu and Z.Z. Hu (2004). Simulation of nonlinear interactions between waves and floating bodies through a finite element based numerical tank. Proc. R. Soc. Lond. Ser. A, 460 2797-2817.

[21] C.Z. Wang and B.C. Khoo (2005). Finite element analysis of two-dimensional nonlinear sloshing problems in random excitations. Ocean Eng., 32, 107-133.

[22] C.Z. Wang and G.X. Wu (2007). Time domain analysis of second order wave diffraction by an array of vertical cylinders. J. Fluids Struct., 23 (4), 605-631.

[23] C.Z. Wang and G.X. Wu (2008). Analysis of second-order resonance in wave interactions with floating bodies through a finite-element method. Ocean Eng., 35, 717-726.

[24] S. Yan and Q.W. Ma (2009). QALE-FEM for modelling 3D overturning waves. Int. J. Numer. Methods Fluids, http://dx.doi.org/10.1002/fld.2100.

[25] H. Song, L. Tao, and S. Chakrabarti (2010). Modelling of water wave interaction with multiple cylinders of arbitrary shape. J. Comput. Phys., 229, 1498-1513.

[26] [ITTC84], Report of Resistance Committee, Proc.17th ITTC, Göteborg (1984).

[27] C. W. Dawson (1977). A practical computer method for solving ship-wave problems. 2nd Int. Conf. Numerical Ship Hydrodynamics, Berkeley, USA.

[28] H. C. Raven (1996). A solution method for the nonlinear ship wave resistance problem. Ph.D. thesis, MARIN, Netherlands.

[29] A. Souto-Iglesias (2001). Nuevas herramientas de diseño de formas de buques basadas en códigos de flujo potencial. Ph.D. thesis, Escuela Técnica Superior de Ingenieros Navales, Madrid.

[30] J.M.J. Journée (1992). Experiments and Calculations on 4 Wigley Hull Forms in Head Waves. Report 0909, Delft University of Technology.

[31] H.Maruo (1957). The excess resistance of a ship in rough seas. International Shipbuilding Progress, 4 (35). 
[32] H. Maruo (1960). The drift of a body floating on waves. Journal of Ship Research, 4 (3), 1-10.

[33] H. Maruo (1963). Resistance in waves, 60th anniversary Series. The Society of Naval Architects of Japan, 8, 67-102.

[34] S. A. G. Joncquez (2009). Second-order forces and moments acting on ships in waves. Ph.D. thesis, Technical University of Denmark, Denmark.

[35] Md. S. Tarafdera and K. Suzuki (2008). Numerical calculation of free-surface potential flow around a ship using the modified Rankine source panel method. Ocean Engineering, 35 536-544.

[36] K.A. Belibassakis, Th.P. Gerostathis, K.V. Kostas, C.G. Politis, P.D. Kaklis, A.I. Ginnis, and C. Feurer (2013). A BEM-isogeometric method for the ship wave-resistance problem. Oceang Engineering, 60 53-67.

[37] J. N. Newman (1977). Marine hydrodynamics. Cambridge: The MIT press.

[38] O. C. Zienkiewicz, R. L. Taylor, and P. Nithiarasu (2005). The finite element method for fluid dynamics. Elsevier Butterworth-Heinemann.

[39] J. Donea and A. Huerta (2003). Finite Element Methods for Flow Problems. Chichester: Wiley.

[40] A. N. Brooks and T. J. R. Hughes (1982). "Streamline upwind/Petrov-Galerkin formulations for convection dominated flows with particular emphasis on the incompressible Navier-Stokes equations," Comput. Methods Appl. Mech. Eng., vol. 32, no. 1-3, pp. 199-259, Sep.

[41] W. L. Wood, M. Bossak, and O. C. Zienkiewicz (1980). An alpha modication of Newmark's method. International Journal for Numerical Methods in Engineering, 15(10):1562-1566.

[42] B. M. Irons, and R. C. Tuck (1969). A Version of the Aitken Accelerator for Computer Iteration. Int. J. Numer. Methods Eng., vol. 1, pp. 275-277.

[43] R. McCamy and R. Fuchs (1954). Wave forces on piles: a diffraction theory, Tech. Memo No. 69, U.S. Army Corps of Engrs.

[44] G. F. Clauss, C. Schmittner, and K. Stutz (2002). Time-domain investigation of a semisubmersible in rouge waves, 21st International Conference on Offshore Mechanics and Arctic Engineering June 23-28, Oslo, Norway. OMAE2002-28450.

[45] R.E. Taylor and ER. Jefferys (1986). Variability of Hydrodynamic Load Predictions for a Tension Leg Platform, Ocean Eng., Vol 13; 5, 449-490.

[46] WAMIT User Manual. http://www.wamit.com/manual.htm.

[47] A. C. Fernandes and L. A. P. Levy (1990). Cálculo de esforços de onda de primeira e segunda ordem em navios e plataformas flutuantes através de integração azimutal analítica, Congresso da SOBENA Río de Janeiro, Rj, Brazil.

[48] K. Kudou (1977). The drifting force acting on a three-dimensional body in waves, J.S.N.A. Japan; Vol. 141. 
[49] J. A. Pinkster (1981). Low frequency second order wave exciting forces on floating structures. Wageningen: Netherlands Ship Model Basin 650.

[50] S.Wojciech (1993). Diffraction of second-order surface waves by semisubmerged horizontal rectangular cylinder. J. Waterway, Port, Coastal, Ocean Eng., Vol 119, 160-171.

[51] J.N. Newman and F.A.P. Poole (1962). The wave resistance of a moving pressure distribution in a canal. Schiffstechnik, 9 (45), 21-26.

[52] J.R. Shearer and J.J. Cross (1965). The experimental determination of the components of ship resistance for a mathematical model.

[53] S. Ju (1983). Study of total and viscous drag for the Wigley parabolic ship form. IIHR Report, no. 261 , p. 35.

[54] R. Aubry, F. Mut, R. Löhner, and J.R. Cebral (2008). Deflated preconditioned conjugate gradient solvers for the Pressure-Poisson equation. J. Comp. Phys., 227: 10196-10208.

[55] F.Mut, R. Aubry, G. Houzeaux, J. Cebral, and R. Löhner (2010). Deflated preconditioned conjugate gradient solvers: extensions and improvements. 48th Aerospace Sciences Meeting and Exhibit, Orlando, FL, January.

[56] F.Mossaiby, R. Rossi, P. Dadvand, and S. Idelsohn (2011). OpenCL-based implementation of an unstructured edge-based finite element convection-diffusion solver on graphics hardware. Int. J. Numer. Meth. Engng., 89, 13: 1635-1651.

[57] N.Bell and M.Garland (2008). Effcient Sparse Matrix-Vector Multiplication on CUDA. NVIDIA Technical Report NVR-2008-004, Dec.

[58] Y. Saad (1996). Iterative Methods for Sparse Linear Systems. PWS Publishing Company, Boston.

[59] R. Chandra. Parallel Programming in OpenMP (2001). Morgan Kaufmann Publishers.

[60] D. J. Donnelly and W. L. Neu (2011). Numerical Simulation of Flow About a Surface-Effect Ship. 11th International Conference on Fast Sea Transportation FAST 2011, Honolulu, Hawai. September 2011.

[61] S. M. Mousaviraad, S. Bhushan, and F. Stern (2012). CFD Prediction of Free-Running SES/ACV Deep and Shallow Water Maneuvering in Calm Water and Waves. MARSIM 2012. Singapore, April 23-27.

[62] B. S. H. Connell, W. M. Milewski, B. Goldman, and D. C. Kring (2011). Single and MultiBody Surface Effect Ship Simulation for T-Craft Design Evaluation. 11th International Conference on Fast Sea Transportation FAST 2011, Honolulu, Hawai. September 2011.

[63] D. C. Kring, M. K. Parish, W. M. Milewski, and B. S. H. Connell (2011). Simulation of Maneuvering in Waves for a High-Speed Surface Effect Ship. 11th International Conference on Fast Sea Transportation FAST 2011, Honolulu, Hawai. September 2011.

[64] L. Yun and A. Bliault (2005). Theory \&Design of Air Cushion Craft, Elsevier. 
[65] N. Hirata and O. M. Faltinsen (2000). Computation of Cobblestone effect with unsteady viscous flow under a stern seal bag of a SES. Journal of Fluids and Structures; 14, 1053-1069.

[66] L. J. Doctors (1975). Nonlinear motion of an air-cushion vehicle over waves. Journal of Hydronautics; 9 (2), 44-57.

[67] P. A. Sullivan, P. A. Charest, and T. Ma (1994). Heave stiffness of an air cushion vehicle bag and finger skirt. Journal of Ship Research; 38 (4), 302-307.

[68] Q. Yang, V. Jones, and L. McCue (2011). Investigation of Skirt Dynamics of Air Cushion Vehicles under Non-linear Wave Impact Using a SPH-FEM Model. 11th International Conference on Fast Sea Transportation FAST 2011, Honolulu, Hawaii. September 2011.

[69] R. L. Taylor, E. Oñate, and P.-A. Ubach (2005). Finite element analysis of membrane structures, Textile composites and inflatable structures. Springer, 47-68.

[70] C. Felippa and B. Haugen (2005). A unified formulation of small-strain corotational finite elements: I. Theory. Computer Methods in Applied Mechanics and Engineering, 194 2285-2335

[71] C. A. Felippa and C. Militello (1992). Membrane triangles with corner drilling freedoms: II. The ANDES element, Finite Elem Anal Des, 12:189-201.

[72] M. A. Crisfield (1997). Non-linear Finite Element Analysis of Solid and Structures. Advanced Topics, vol. 2, Wiley.

[73] F. S. Almeida and A. M. Awruch (2011). Corotational nonlinear dynamic analysis of laminated composite shells. Finite Elements in Analysis and Design, 47 1131-1145.

[74] A. Ibrahimbegovic and S. Mamouri (2002). Energy conserving/decaying implicit time-stepping scheme for nonlinear dynamics of three-dimensional beams undergoing finite rotations. Computer Methods in Applied Mechanics and Engineering, 191 4241-4258.

[75] C. A. Felippa, K. C. Park and C. Farhat (2001). Partitioned analysis of coupled mechanical systems. Comput. Methods Appl. Mech. Eng., vol. 190, pp. 3247-3270.

[76] J. G. Valdés (2007). Nonlinear analysis of orthotropic membrane and shell structures including fluid-structure interaction. PhD thesis, Barcelona Tech.

[77] S. F. Zalek and L. J. Doctors (2010). Experimental Study of the Resistance of Surface-EffectShip Seals. Proceeding of the 28th Symposium on Naval Hydrodynamics.

[78] R. J. Paredes (2013). Smoothed particle hydrodynamics applied to fluid structure interaction problems involving hydroelastic response. Ph.D. thesis, Stevens Institute of Technology, United States of America.

[79] A. D. Wiggins, S. F. Zalek, M. Perlin and S. L. Ceccio (2011). Development of large scale surface effect ship bow seal testing plataform. 11th International Conference on Fast Sea Transportation FAST 2011, Honolulu, Hawai. September 2011. 
[81] M.A.S. Neves, J.A. Merino, and C.A. Rodríguez (2009). A nonlinear model of parametric rolling stabilization by anti-roll tanks. Ocean Engineering, 36(14):1048-1059.

[82] R.A. Watai, P. Dinoi, F. Ruggeri, A. Souto-Iglesias, and A.N. Simos (2015). Rankine timedomain method with application to side-by-side gap flow modeling. Applied Ocean Research, 50(0):69-90.

[83] W.H. Zhao, J.M. Yang, Z.Q. Hu, and Y.F. Wei (2011). Recent Developments on the Hydrodynamics of Floating Liquid Natural Gas (FNLG). Ocean Engineering, 38:1555-1567.

[84] W. Zhao, J. Yang, Z. Hu, and L. Tao (2014). Coupled analysis of nonlinear sloshing and ship motions. Applied Ocean Research, 47(0):85-97.

[85] Y. Kim, B.W. Nam, D.W. Kim, and Y.S. Kim (2007). Study on coupling effects of ship motion and sloshing. Ocean Engineering 34, 2176-2187.

[86] Y. Long LI, R. chuan ZHU, G. ping MIAO, and J. Fan (2012). Simulation of tank sloshing based on OpenFOAM and coupling with ship motions in time domain. Journal of Hydrodynamics, Ser. B, 24(3):450-457.

[87] Q.W. Ma and S. Yan (2009). QALE-FEM for numerical modelling of non-linear interaction between 3D moored floating bodies and steep waves. International Journal for Numerical Methods in Engineering, 78(6):713-756,

[88] S.Yan, Q.W. Ma, and X. Cheng (2011). Fully nonlinear simulation of two floating structures in close proximity subjected to oblique waves. In 21 st International Offshore and Polar Engineering Conference (ISOPE). The International Society of Offshore and Polar Engineers (ISOPE).

[89] B.Bouscasse, A. Colagrossi, A. Souto-Iglesias, and J.L. Cercos-Pita (2014). Mechanical energy dissipation induced by sloshing and wave breaking in a fully coupled angular motion system. I. theoretical formulation and numerical investigation. Physics of Fluids (1994-present), 26(3).

[90] B.Bouscasse, A. Colagrossi, A. Souto-Iglesias, and J.L. Cercos-Pita (2014). Mechanical energy dissipation induced by sloshing and wave breaking in a fully coupled angular motion system. II. experimental investigation. Physics of Fluids (1994-present), 26(3).

[91] G.Bulian, A.Souto-Iglesias, L. Delorme, and E. Botia-Vera (2010). SPH simulation of a tuned liquid damper with angular motion. Journal of Hydraulic Research, 48(Extra Issue):28-39,.

[92] A. Herault, G. Bilotta, and R.A. Dalrymple (2010). SPH on GPU with CUDA. Journal of Hydraulic Research, 48(Extra Issue):74-79.

[93] A. Crespo, J. Dominguez, A. Barreiro, M. Gómez-Gesteira, and B. Rogers (2011). GPUs, a new tool of acceleration in CFD: Efficiency and reliability on smoothed particle hydrodynamics methods. PLoS ONE, 6(6).

[94] J.M. Dominguez, A. J.C. Crespo, D. Valdez-Balderas, B. D. Rogers, and M. Gómez-Gesteira (2013). New multi-GPU implementation for smoothed particle hydrodynamics on heterogeneous clusters. Computer Physics Communications, 184:1848-1860. 
[95] D. Nishiura, M. Furuichi, and H. Sakaguchi (2015). Computational performance of a smoothed particle hydrodynamics simulation for shared-memory parallel computing. Computer Physics Communications, 194(0):18-32,

[96] J.L. Cercos-Pita (2015). Aquagpusph, a new free 3D SPH solver accelerated with OpenCL. Computer Physics Communications, 192(0):295-312.

[97] J.J. Monaghan (1994). Simulating free surface flows with SPH. Journal of Computational Physics, 110:399-406.

[98] A. Colagrossi, M. Antuono, and D. Le Touzé (2009). Theoretical considerations on the freesurface role in the smoothed-particle-hydrodynamics model. Physical Review E (Statistical, Nonlinear, and Soft Matter Physics); 79(5):056701.

[99] A. Colagrossi, M. Antuono, A. Souto-Iglesias, and D. Le Touzé (2011). Theoretical analysis and numerical verification of the consistency of viscous smoothed-particlehydrodynamics formulations in simulating free-surface flows. Physical Review E, 84:26705.

[100] A. Colagrossi, A. Souto-Iglesias, M. Antuono, and S. Marrone (2013). Smoothed-particlehydrodynamics modeling of dissipation mechanisms in gravity waves. Phys. Rev. E, 87:023302.

[101] J.J. Monaghan (2012). Smoothed particle hydrodynamics and its diverse applications. Annual Review of Fluid Mechanics; 44(1):323-346.

[102] AQUAgpusph: http://canal.etsin.upm.es/aquagpusph/

[103] J.L. Cercos-Pita, L.M. Gonzalez, A. Moreno, A. Guerrero, and S. Salgado (2014). Simulation of earthquake sloshing loads in a nuclear reactor. In 9th International SPHERIC SPH Workshop.

[104] Tcl (Tool Command Language) [on-line] http://www.tcl.tk

[105] B. Molin, F. Remy, S. Rigaud, and Ch.de Jouette (2002). LNG-FPSO's: frequency domain coupled analysis of support and liquid cargo motions. Proceedings of INAM Conference, Rethymnon, Greece.

[106] A. Ludvigsen, Z. Y. Pan, P. Gou, and T. Vada (2013). Adapting a linear potential theory solver for the outer hull to account for fluid dynamics in tanks. Proceedings of the ASME 32nd International Conference on Ocean, Offshore and Arctic Engineering OMAE2013 June 9-14, Nantes, France.

[107] K. J. Bai and K. P. Rhee (1987). Roll-damping tank test, Seoul National University, Project report.

[108] Y.Kim (2002). A numerical study on sloshing flows coupled with ship motion - the antirolling tank problem. Journal of Ship Research, 46 (1), pp. 52-62.

[109] http://canal.etsin.upm.es/papers/servanetal2016/

[110] W. Zhao, J. Yang, Z. Hu, L. Xiao, and L. Tao (2014). Hydrodynamics of a 2D vessel including internal sloshing flows. Ocean Engineering, 84: 45-53. 
[111] SP. Breton and H. Moe (2009). Status, plans, and technologies for offshore wind turbines in Europe and North America. Renew Energy.,34:646-54.

[112] M. Karimirad, Q. Meissonnier, Z. Gao, and T. Moan (2010). Hydroelastic code-to-code comparison for a tension leg spar-type floating wind turbine. Marine Struct., 24:412-435.

[113] S.H. Jeon, Y.U. Cho, M.W. Seo, and J.R. Cho, W.B. Jeong (2013). Dynamic response of floating substructure of spar-type offshore wind turbine with catenary mooring cables. Ocean Eng., $72: 356-364$.

[114] P. Sclavounos, S. Buttereld, W. Musial, and J.M. Jonkman (2005). Engineering challenges for floating offshore wind turbines. In Proc: Copenhagen Offshore Wind Conference.

[115] A. Cordle and J.M. Jonkman (2011). State of the art in floating wind turbine design tools. In Proc: 21st International Offshore and Polar Engineering Conference (ISOPE 2011).

[116] D. Matha, M. Schlif, A. Cordle, R. Pereira, and J.M. Jonkman (2011). Challenges in simulation of aerodynamics, hydrodynamics, and mooring-line dynamics of floating offshore wind turbines. In Proc: 21st International Offshore and Polar Engineering Conference (ISOPE 2011).

[117] P.B. Jacob, R.A. Bahiense, F.N. Correa, and B.M. Jacovazzo (2012). Parallel implementations of coupled formulations for the analysis of floating production systems, part I: Coupling formulations. Ocean Eng., 55: 206-218.

[118] P.B. Jacob, L.D. Franco, M.V. Rodrigues, F.N. Correa, and B.M. Jacovazzo (2012). Parallel implementations of coupled formulations for the analysis of floating production systems, part II: Domain decomposition strategies. Ocean Eng., 55 :219-234.

[119] Y.H. Bae and M.H. Kim (2013). Rotor-floater-tether coupled dynamics including second-order sum-frequency wave loads for a mono-column-TLP-type FOWT (Floating Offshore Wind Turbine). Ocean Eng. 2013, 61 :109-122.

[120] Y.M. Low and R.S. Langley (2006). Time and frequency domain coupled analysis of deepwater floating production systems. Appl. Ocean Res. 2006, 28 :371-385.

[121] ABS (2012). Floating Wind Turbines. American Bureau of Shipping. Bureau of Safety and Environmental Enforcement. Retrieved from http://www.offshorewindhub.org/.

[122] Y.M. Low (2008). Prediction of extreme responses of floating structures using a hybrid time/frequency domain coupled analysis approach. Ocean Eng., 35 :1416-1428.

[123] J. E. Gutierrez-Romero (2014). Desarrollo de heramientas software para el análisis de aerogeneradores "offshore" sometidos a cargas acopladas de viento y oleaje. Ph. D. Thesis, Technical University of Cartagena.

[124] (www.ieawind.org/task23/)

[125] J.M. Jonkman, S. Butterfield, W. Musial, and G. Scott (2009). Definition of a 5-MW reference wind turbine for offshore system development. Technical Report NREL/TP-500-38060. National Renewable Energy Laboratory, www.nrel.gov, Colorado (USA).. 
[126] J.M. Jonkman (2010). Definition of the floating system for Phase IV of OC3. Technical Report NREL/TP-500-47535. National Renewable Energy Laboratory, www.nrel.gov, Colorado (USA).

[127] J.M. Jonkman (2005). Buhl Jr ML. FAST's Users Guide. Technical Report NREL/EL-50038230. National Renewable Energy Laboratory, www.nrel.gov, Colorado (USA).

[128] J.E. Gutiérrez-Romero, B. Zamora, J. García-Espinosa, and R.Peyrau (2013). Tool development based on FAST for performing design optimization of offshore wind turbines: FASTLognoter. Renew. Energy., 55:69-78.

[129] B.J. Jonkman and L.Kilcher (2012). Turbsim User's Guide. Version 1.06.00. Technical Report NREL/TP-xxx-xxxxx. National Renewable Energy Laboratory, www.nrel.gov, Colorado (USA).

[130]J.M. Jonkman (2007). Dynamics Modeling and Loads Analysis of an Offshore Floating Wind Turbine. Technical Report NREL/TP-500-41958. National Renewable Energy Laboratory, www.nrel.gov, Colorado (USA).

[131] G.C.V. Ramanchandran, A. Robertson, J.M. Jonkman, and M.D. Masciola (2013). Investigation of Response Amplitude Operators for Floating O_shore Wind Turbines. In Proc: 23rd International Ocean, O_shore and Polar Engineering Conference (ISOPE 2013).

[132] http://www.compassis.com/compass/es/Productos/SeaFEM.

[133] Pierson J.P. and Moskowitz L. (1964). A Proposed Spectral Form for Fully Developed Wind Seas Based on the Similarity Theory of S.A.Kitaigorodskii. Journal of Geophysical Research, Vol. 69, No.24.

[134] Faltinsen O.M. (1998). Sea loads on ships and offshore structures, Cambridge Ocean Technology Series.

[135] Hasselmann K., Barnett R.C., Bouws E., Carlson H., Cartwright D.E., Enke K., Ewing J.A., Gienapp H., Hasselmann D.E., Kruseman P., Meerburg A., Müller P., Olbers, D.J., Richter K., Sell W., Walden H. (1973). Measurements of Wind-Wave Growth and Swell Decay during the Joint North Sea Wave Project (JONSWAP). Deutches Hydrographisches Institut, No.12. 